\title{
CATALOGUE SYSTÉMATIQUE
}

\section{DES NOMS DE GENRES}

\section{DE POISSONS ACTUELS}

de la $\mathrm{X}^{\circ}$ édition du «Systema naturae 》de Charles LINNÉ jusqu'à la fin de l'année 1959

par

Yves-J. GOLVAN

Institut de Parasitologie de la Faculté de Médecine de Paris 
«Ne sint nomina et perit cognitio rerum.

Ch. LINNÉ.

La Systématique n'est une Science ni mineure ni obscure. Lorsqu'une fois pour tontes on s'est donné la peine de lire et d'assimiler les quelques lois simples qui la régissent, on se trouve en possession d'un instrument de travail remarquablement souple et qui assure une compréhension aisée quelle que soit la langue maternelle de l'interlocuteur.

Pour le Parasitologiste, la Systématique a une importance majeure puisqu'il doit tenir compte non seulement du parasite, mais de son hôte. Ce dernier est si primordial que nous connaissons nombre de nos collègues qui se refusent à étudier des parasites dont on ne sait sur ou dans quel animal-hôte ils furent récoltés. Qui dit connaissance de l'hôte dit ipso-facto connaissance précise de son nom. Si l'on se contente, en ce domaine, d'une approximation, on s'expose d̀ des erreurs considérables. C'est pour avoir, volontairement ou non, méconnu ce principe de base que bien des extrapolations traitant de problèmes «philosophiques» majeurs comme la Spécificité parasitaire, l'Adaptation des Parasites à leurs hôtes, la Biogéographie on l'Evolution des complexes xéno-parasitaires se trouvent, dès le départ, erronées.

Or les listes d'hôtes dont nous disposons contiennent de très nombreuses erreurs manifestes et l'on peut en inférer qu'il $y$ existe un bien plus grand nombre d'inexactitudes plus subtiles. D'où proviennent ces erreurs? Qui en est responsable? Nous pouvons tous nous sentir coupables, depuis le premier chercheur qui a capturé l'hôte jusqu'au dernier; qui a réuni sous sa propre signature plusieurs compilations antérieures. Et c'est ainsi que l'on voit figurer dans une liste de parasites de Mammifères un parasite de Raie qui s'y est égaré par des voies mystérieuses, on tel Helminthe d'un Tatou sud-américain élire fortuitement domicile chez un Hérisson d'Afrique centrale. Fréquent est également le cas de l'auteur qui, croyant que tous partagent ses préoccupations immédiates, indique le nom latin de l'hôte sans même préciser à quelle classe il appartient. Il nous est arrivé ainsi de découvrir, non sans stupeur, que tel binôme cachait un Coléoptère ou une Blatte, alors que nous croyons avoir affaire à un quelconque Vertébré.

Certes ces erreurs grossières sont faciles à redresser lorsque l'on a la chance de travailler dans un centre important, de disposer d'une documentation abondante, d'avoir un accès facile à une grande bibliothèque et même, ce qui est notre cas, de pouvoir à tout moment demander aide et assistance à nos collègues du Muséum d'Histoire Naturelle. Ces der- 
niers nous ont toujours tiré d'embarras avec beaucoup d'empressement et d'amabilité et c'est pourquoi nous nous faisons scrupule de les importuner trop souvent au cours de leur travail.

Mais, hors de ces endroits privilégiés, le problème de la place de l'hôte dans l'échelle zoologique devient rapidement insoluble. Il existe bien des Index, tels le Nomenclator zoologicus de S. A. Neave, mais, outre qu'il faille d'abord les posséder, ils donnent des renseignements insuffisants. Ils vous diront, par exemple, que Penthetor est un Mammifère, mais non qu'il s'agit d'une Chauve-Souris. Qui plus est l'index n'indique souvent pas si tel nom de genre est attribué à un animal vivant ou fossile, si tel autre est actuellement valide et, s'il ne l'est plus, par quoi il se trouve remplacé dans la nomenclature d'aujourd'hui.

C'est pour tenter de pallier cette insuffisance d'information que nous avons entrepris la rédaction de ce « Réperioire des noms de genres de Vertébrés actuels» d'abord pour notre usage personnel. Nous avons ensuite pensé qu'il venait combler une importante lacune, qu'il était souhaitable qu'il fût imprimé sous cette forme simple afin qu'il puisse servir quotidiennement à nos collègues Parasitologistes, sans entraîner une trop importante dépense. Ce faisant, nous n'avons nullement eu la prétention de faire cuvre originale, de nous aventurer sur un terrain qui n'est pas le nôtre, d'oser introduire en ce domaine qui nous est étranger des innovations de notre cru, en un mot d'agir en spécialiste des Vertébrés, ce que nous ne sommes nullement.

Ce travail ne prétend pas être complet non plus qu'exempt d'erreurs, mais d'ores et déjà il s'est avéré une source d'informations facile à consulter et généralement suffisante pour notre propos.

De toute évidence il fallait adopter une Classification des Vertébrés qui puisse servir de référence de base et qui soit facilement contrôlable si besoin en était. Nous avons pensé que le plus simple était d'adopter comme canevas la Systématique utilisée par les différents auteurs des chapitres taxinomiques figurant dans le «Traité de Zoologie» publié sous la direction du Professeur P.-P. Grassé et édité chez Masson et Cie à Paris. La diffusion de ce travail monumental très récent mais déjà classique, nous autorise à croire qu'il sera aisément accessible à tous ceux qui désireront des informations moins sommaires que celles que nous donnons ici.

Dans un premier essai de réalisation de ce répertoire, nous avions délibérément omis les noms d'auteurs des genres cités et la date de création de ces noms. Nous avons très vite renoncé à cette solution de facilité car nombre de genres ont été utilisés par plusieurs auteurs, voire par le même, pour désigner des animaux fort différents. Nous n'avons indiqué la mise en synonymie que lorsqu'elle était dûment consacrée par, l'usage. Que l'on veuille bien nous pardonner d'avoir ainsi conservé un trop grand nombre de genres désuets et inutiles. Les « Annales de Parasitologie humaine et comparée» viennent de publier notre « Catalogue 
des genres de Poissons actuels» allant de la $X^{\bullet}$ édition du Systema naturae de Charles Linné à 1959 et de commencer l'impression du «Catalogue des espèces et sous-espèces de Mammifères actuels du Monde». Ainsi sera complété et partiellement corrigé le présent travail.

Dans ce Catalogue les genres sont placés par ordre alphabétique, suivis de leur nom d'auteur et de leur date de création. Les principales synonymies sont indiquées et renvoient au genre valide. L'utilisation pratique du travail est simple. Nous indiquons d'abord la Classe par les lettres conventionnelles suivantes:

- C. pour Cyclostome,

- P. pour Poisson,

- Amph. pour Amphibien,

- R. pour Reptile,

- Av. pour Oiseau,

- M. pour Mammifère.

Nous donnons ensuite, toujours, l'Ordre en lettres capitales, puis la famille en italiques. Lorsque besoin en était, l'Ordre est suivi du Sousordre placé entre parenthèses et parfois la Famille est complétée par la désignation de la Sous-famille également placée entre parenthèses. C'est ainsi que, par exemple:

Chinchillula Thomas 1898, : M.-Roden. (Myomorph.) Cricetid. (Hesperomyin.) doit se lire : Mammifère appartenant à l'Ordre des RODENTIA, au Sous-ordre des Myomorpha, à la Famille des Cricetidae et à la Sous-famille des Hesperomyinae.

Chirocentrodon Günther 1868 : P.-Cluperf. (Clupeoid.) Chirocentrid. doit s'entendre: Poisson appartenant à l'Ordre des CLUPEIFORMES, au sous-ordre des CLUPEOIDEI, à la famille des Chirocentridae.

Nous souhaitons que ce Catalogue, tel qu'il est, serve quotidiennement à tous nos collègues non seulement Parasitologistes, mais Zoologistes, quel que soit la discipline ou le groupe auquel ils s'intéressent. Si cette ambition se réalise, nous estimerons avoir pleinement atteint le but que nous nous proposions lorsque, il y a quelques années, nous avons entrepris ce travail. Nous serons heureux d'avoir ainsi pu rendre bien modestement service à tous.

Y.-J. G.

Institut de Parasitologie de la Faculté de Médecine de Paris, (Directeur : L.-Ch. BRumpt). 



\section{Sous-classe des Cyclostomes (Cyclostomia)}

Ordre des MYXINIFORMES

FAMILLE DES MYXINIDAE

(Myxines ; Hag-fishes)

Anopsus RAFINESQUe 1815: syn. de Myxine L. 1758.

Dodecatrema FOWLER 1948.

Gastrobranchus BLOCH 1797.

Muraenoblenna DE LACÉPÈDE 1803 : syn. de Myxine L. 1758.

Myxine LINNÉ 1758.

Nemamyxine RICHARDSON 1958.

Neomyxine RICHARDSON 1953.

Notomyxine NANI et GNERI 1952.

\section{FAMILLE DES EPTATRETIDAE} (Bdellostomes)

Bdellostoma Müller 1835 : syn. d'Eptatretus C. 1819.

Eptatretus Cloquet 1819.

Heptatrema DUMÉRIL 1856 : syn. d'Eptatretus C. 1819.

Heptatretus REgAN 1912: syn. d'Eptatretus C. 1819.

Hexabranchus SCHULTZE 1835.

Homea Fleming 1822.

Paramyxine DEAN 1904.

Polistotrema GILL 1881.

\section{Ordre des PETROMYZONIFORMES}

FAM. DES PETROMYZONIDAE (Lamproies ; Lampreys)

Agnathomyzon Gracianov 1906 : syn. de Caspiomyzon B. 1906.
Anumocoetes (D.) CUVIER 1817 : syn. d'Ammocoetus D. 1808.

Ammocoetus DUMÉRIL 1808: syn. de Petromyzon L. 1758.

Bathymyzon GILL 1883.

Caspiomyzon BERG 1906.

Entosphenus GILL 1862.

Eudontomyzon REGAN 1911.

Haploglossa Gracianov 1906: syn. de Caspiomyzon B. 1906.

Ichthyomyzon GIRARD 1859.

Lampedra RAFINESQUE 1815: non valide.

Lempetra GRAY 1851.

Lethenteron CREASER et HUBBS 1922: s./g. de Lampetra G. 1851.

Oceanomyzon FOWLER 1908.

Okkelbergia CREASER et HuBBS 1922: s./g. de Lampetra G. 1851.

Petromyzon LINNÉ 1758.

Pricus RAFINESQUe 1815: non valide.

Reighardina CREASER et HubBS 1922 : s./g. d'Ichthyomyzon G. 1859 .

Scolecosoma GIRARD 1859 : larve d'Ichthyomyzon G. 1859.

Tetrapleurodon CREASER et HuBBS 1922: s./g. de Lampetra G. 1851.

\section{FAMILLE DES GEOTRIIDAE}

$$
\text { (= Mordaciidae) }
$$

Caragola Gray 1851.

Carangola: errat. pro Caragola G. 1851. 
Chilopterus PhILIPPI 1858: larve de Caragola G. 1851.

Exomegas BURMEISTER 1868.

Geotria Gray 1851.

Macrophthalmia Plate 1897. Mordacia Gray 1851 : syn. de Caragola G. 1851 .
Neomordacia DE CAstelnau 1872: syn. de Geotria G. 1851.

Thysanochilus PHILIPPI 1857: non valide, syn. de Velasia G. 1851.

Velasia Gray 1851.

Yarra DE CASTelnaU 1872: syn. de Geotria G. 1851. 


\section{Classe des Poissons (Piscea)}

\section{Chondricbtbyes}

\section{Sous-classe des Selaciens (Selachia)}

SERIE DES PLEUROTREMATA

Ordre des HEXANCHIFORMES

FAMILLE DES HEXANCHIDAE

(Griset et Perlon; Cow-sharks)

Heptanchus Müller et Henle 1839 : syn. d'Heptranchias R. 1810.

Heptranchias RAFINESQUe 1810.

Hexanchus RAFINESQUE 1810.

Holodus AgAssiz 1842: non valide, syn. d'Heptranchias R. 1810.

Monopterhinus DE BLAINVILle 1816 : syn. d'Hexanchus R. 1810.

Notidanus CUVIER 1817: syn. d'Hexanchus R. 1810.

Notorhynchus AYRES 1855.

\section{FAMILLE DES}

CHLAMYDOSELACHIDAE

(Requin-frangé ; Frilled Shark)

Chlamydoselachus GARMAN 1884.

Cladoselache DEAN 1894: syn. de Chlamydoselachus G. 1884.

\section{Ordre des HETERODONTIFORMES}

FAMILLE DES HETERODONTIDAE

(= Cestracionidae) (Requin de PortJackson ; Bull-head Shark)

Centracion Gray 1831: syn. d'Heterodontus $B .1816$.
Cestracion KLEIN 1775 : non valide. Cestracion Cuvier 1817: syn. d'Heterodontus B. 1816.

Gyropleurodus GILL 1862.

Heterodontus DE BLAINVILLE 1816.

Molochophrys WHITLEY 1931.

Oroptychus Agassiz 1846: non valide.

Tropidodus GiLL 1862.

Wuia FOWLER 1934 : s./g. d'Heterodontus B. 1816.

\section{Ordre des GALEIFORMES}

Sous-ordre des ISUROIDEI

FAMILLE DES ALOPIIDAE (Renards de mer)

Alopecias Müller et Henle 1838: syn. d'Alopias R. 1810.

Alopias RAFINESQUe 1810.

Vulpecula VALMONT 1768: non valide.

Vulpecula (V.) GARMAN 1913: syn. d'Alopias R. 1810.

FAMILLE DES CETORHINIDAE (Pèlerins ; Basking-sharks)

Cetorhinus de Blainville 1816.

Halsydrus Fleming 1817: syn. de Cetorhinus B. 1816. 
Polyprosopus Couch 1862: syn. de Cetorhinus B. 1816.

Selache Cuvier 1817: syn. de Cetorhinus B. 1816 .

Tetroras RAFINESQUE 1810: non valide.

\section{FAMILLE DES \\ GINGLYMOSTOMIDAE \\ (Requin-nourrice)}

Ginglymostoma MüLLER et HeNLE 1837.

Nebrius Rüppel 1835 : serait préemployé, syn. de Nebrodes G. 1913.

Nebrodes Garman 1913 : nom. nov. pro Nebrius R. 1835.

FAMILLE DES ISURIDAE

(= Lamnidae) (Requin-bleu et Lanie

Mackerel-sharks or Man-eaters)

Carcharocles JORDAN et HANNIBAL 1923.

Carcharodon Müller et Henle 1838.

Exoles Gistel 1848 : syn. de Lamna C. 1817 .

Glaphyrodus AgAssiz 1846 : non valide, syn. d'Isurus R. 1810.

Isuropsis GILL 1861.

Isurus RAFINESQUE 1810.

Lamia Risso 1826 : syn. de Lamna C. 1817 .

Lamna CUVIER 1817.

Oxyrhina Agassiz 1846: syn. de Isurus R. 1810.

Plectrostoma GISTEL 1848.

Selanonius Fleming 1828: syn. de Lamna C. 1817.

\section{FAMILLE DES \\ MITSUKURINIDAE \\ (Goblin-shark)}

Mitsukurina JORDAN 1898.
FAMILLE DES ODONTASPIDAE (= Carchariidae) (Sand-sharks)

Carcharias RAFINESQUE 1810: syn. d'Odontaspis A. 1836.

Eugomphodus GILL 1864 : syn. d'Odontaspis A. 1836.

Odontaspis AgAssiz 1836.

Parodontaspis WHITE 1931: s./g. d'Odontaspis A. 1836.

Synodontaspis WHITE 1931: s./g. d'Odontaspis A. 1836.

Triglochis Müller et Henle 1838 : syn. d'Odontaspis A. 1836.

FAMILLE DES ORECTOLOBIDAE

(Requins-tapis et requins zébrés)

Brachaelurus OGILbY 1906.

Cirriscyllium OGILBy 1908: syn. de Brachaelurus O. 1906.

Crossorhinus Müller et Henle 1838: syn. d'Orectolobus B. 1837.

Eucrossorhinus REGAN 1908.

Heteroscyllium REGAN 1908.

Neoparascyllium WHILEY 1939 :

s./g. de Parascyllium G 1861.

Orectolobus BONAPARTE 1837.

Parascyllium GILL 1861.

Stegostoma Müller et Henle 1837.

Sutorectus WHITLEY 1939.

Zev WHITLEY 1927.

\section{FAMILLE DES \\ PSEUDOTRIAKIDAE}

Pseudotriakis CAPELlo 1868.

\section{FAMILLE DES}

RHINEODONTIDAE

(= Rhincodontidae) (Requin-baleine)

Rhincodon SмIтH 1829: errat. pro.

Rhineodon S. 1829.

Rhineodon SMITH 1829.

Rhinodon (S.) Müller et Henle 1837 : emend. pro. Rhineodon S. 1829 .

Micristodus GILl 1865 : syn. de Rhineodon S. 1829. 


\section{Sous-ordre des CARCHARHINOIDEI}

\section{FAMILLE DES \\ CARCHARHINIDAE}

$(=$ Galeidae $=$ Galeorhinidae $)$

(Chiens de mer; gray-sharks)

Aodon DE LACÉPÈDE 1798 : non valide.

Aprion Müller et Henle 1838: pré-employé, syn. d'Aprionodon G. 1861.

Aprionodon Girl 1861.

Bogimba Whitley 1943: s./g. de Galeolamna O. 1853.

Boreogaleus GiLl 1861.

Calliscyllium TANAKA 1912.

Carcharhias CUvier 1817: syn. de Carcharhinus B. 1816.

Carcharhinus DE BLAINVILLE 1816.

Chaenogaleus GILL 1861.

Cynias Gill 1903.

Cynocephalus KLEIN 1777: non valide.

Cynocephalus (K.) GILl 1861 : préemployé, syn. de Prionace C. 1849.

Dirrhizodon KLUNZINGER 1870.

Emissola JAROCKI 1822 : syn. de Mustelus L. 1790.

Eridacnis SMITH 1913.

Eugaleus GILL 1864: pré-employé, syn. de Galeorhinus B. 1816.

Eulamia Glll 1861: syn. de Carcharhinus B. 1816.

Fur Whitley 1943 : syn. de Furgaleus W. 1953.

Furgaleus WhITLEY 1953.

Galeocerdo MüLler et Henle 1837.

Galeolamna OWEN 1853.

Galeolamnoides WHITLEY 1934: s./g. de Galeolamna O. 1853.

Galeorhinus DE BLAINVILLE 1816.

Galeus VALMONT 1768: non valide.

Galeus KLEIN 1775 : non valide.

Galeus RAFINESQUE 1810: syn. de Mustelus L. 1790.

Galeus Cuvier 1817 : syn. de Galeorhinus $\mathrm{B} .1816$.
Gillisqualus WhitLey 1934.

Hemigaleops Schultz et Welander 1953.

Hemigaleus BLEEKER 1852: pré-employé, syn. de Chaenogaleus G. 1861.

Hemitriakis Herre 1923.

Hypogaleus SMITH 1958.

Hypoption Müller et Henle 1838.

Hypoprionodon GILL 1861.

Isogomphodon GILL 1861.

Isoplagiodon GII.L 1861.

Lamiopsis GILL 1861.

Lamnarius WHITLEY 1943: s./g. de Galeolamna O. 1853.

Leptocarcharias (S.) GüNTHER 1870 : syn. de Leptocarias S. 1837.

Leptocarias SмIтH 1837.

Leptocharias MüLleR et HeNle 1837: syn. de Leptocarias S. 1837.

Longmania WHITLEY 1939

Loxodon Müller et Henle 1838.

Mapolamia WHITLEY 1934.

Mustellus FISCHER 1813: syn. de Mustelus L. 1790.

Mustelus Valmont 1768 : non valide.

Mustelus LINCK 1790.

Mustelus CUvier 1817 : syn. de Mustelus L. 1790.

Myrmillo Gistel 1848 : syn. de Mustelus L. 1790.

Mystidens WhitLey 1944.

Negaprion WhITLEY 1940.

Negogaleus WhItLEY 1931: syn. de Chaenogaleus G. 1861.

Neotriakis SMITH 1957.

Notogaleus Whitley 1931: syn. de Galeorhinus B. 1816.

Ogilamia WhItLEY 1939: s./g. de Galeolamna O. 1853.

Paragaleus BUDKER 1935.

Physodon Müller et Henle 1838.

Platypodon GILI. 1861.

Pleuracromylodon GILL 1864: syn. de Mustelus L. 1790.

Prionace CANTOR 1849.

Prionodon Müller et Henle 1838 : syn. de Prionace C. 1849.

Pterolamia Brenning 1942. 
Pterolamia SprInger 1950: syn. de Pterolamiops S. 1951.

Pterolamiops SPRINGER 1951.

Rhinotriacis GILL 1862.

Rhizoprion OGILBY 1915.

Rhizoprionodon WHITLEY 1928.

Scoliodon Müller et Henle 1837 : s./g. de Carcharhinus B. 1816.

Scylliogaleus BoUlenger 1902.

Thalassinus Moreau: syn. de Prionace C. 1849.

Thalassorhinus MüLler et HENLE Triaenodon MüLleR et Henle 1838. Triakis Mülleer et Henle 1838.

Uranga WHITLEY 1943.

Uranganops WHITLEY 1943: s./g. de Galeolamna O. 1853.

FAMILLE DES SPHRYNIDAE (Requins-marteaux ; Hammer-head Sharks)

Cestracion (K.) Gill 1861 : syn. de Sphyrna R. 1810.

Cestracion (Walbaum) OgILby 1916 : syn. de Sphyrna R. 1810.

Cestrorhinus DE BLAINVILLE 1816: syn. de Sphyrna R. 1810.

Eusphyra GILL 1861.

Platysqualus Swainson 1839: syn. de Sphyrna R. 1810.

Protozygaena Whitley 1940.

Reniceps GILL 1861.

Sphyra VAN DER HOEVEN 1849: emend. pro Sphyrna R. 1810.

Sphyrichthys THIENEMANN 1828: syn. de Sphyrna R. 1810.

Sphyrna RAFINESQUE 1810.

Sphyrnias RAFINESQUE 1815 : syn. de Sphyrna R. 1810.

Zygaena Cuvier 1817: syn. de Sphyrna R. 1810.

\section{FAMILLE DES \\ HEMISCYLLIIDAE}

Chiloscyllium MüLler et Henle 1838.

Cirrhoscyllium SмІтн 1913.
Hemiscyllium SMITH 1837.

Synchismus GiLl 1861: syn. de Chiloscyllium M. et H. 1838.

FAMILLE DES

SCYLLIORHINIDAE

(Roussettes ; Cat-sharks)

Apristurus Garman 1913.

Asymbolus Whitley 1939: s./g. de Scylliorhinus B. 1816.

Atelomycterus Garman 1913.

Aulohalaelurus FOWLER 1934: s./g. d'Halaelurus G. 1861.

Catulus Valmont 1768: non valide.

Catulus SмIтH 1837 : syn. de Scylliorhinus B. 1816.

Catulus (V.) Garman 1913 : pré-employé, syn. de Scylliorhinus B. 1816.

Cephaloscyllium GILL 1861.

Cephalurus Bigelow et SCHROEder 1941.

Conoporoderma FOWLER 1934 : s./g. de Poroderma S. 1837.

Figaro Whitley 1928: s./g. de Pristiurus M. et H. 1838.

Halaelurus GILL 1861.

Haploblepharus GARMAN 1913.

Holohalaelurus FOWLER 1934: s./g. d'Halaelurus G. 1861.

Juncurus WhITLEY 1939: s./g. de Scylliorhinus B. 1816.

Parapristiurus FowLER 1934 : s/g. de Pristiurus M. et H. 1838

Parmaturus Garman 1906.

Poroderma SмITH 1837.

Pristidurus BONAPARTE 1839: non valide.

Pristiurus Müller et Henle 1838.

Pristiurus Bonaparte 1841: syn. de Pristiurus M. et H. 1838.

Proscyllium Hilgendorf 1904.

Scylliorhinus DE BL.AINVIL.LE 1816.

Scyllium Cuvier 1817 : syn. de Scylliorhinus B. 1816. 
FAMILLE DES PENTANCHIDAE

Caninoa NARDo 1844 : validité douteuse.

Caninotus NARDo 1844 : validité douteuse.

Pentanchus SмIтH 1912.

Thalassoklephtes GISTEL 1848: syn. de Caninoa N. 1844.

\section{Ordre des SQUALIFORMES}

\section{Sous-ordre des SQUALIOIDEI}

FAMILLE DES SQUALIDAE $(=$ Dalatiidae $)$

(Requins-épineux et Requin-chagrin ; Dog-fishes)

Acanthias Risso 1826.

Acanthias Bonaparte 1845 : syn. de Squalus L. 1758.

Acanthidium Lowe 1839 : syn. d'Etmopterus R. 1810.

Acanthorhinus DE BLAINVILle 1816 : syn. de Squalus L. 1758.

Atractophorus Gilchrist 1922 : syn. de Dalatias R. 1810.

Carcharias GISTEL 1848: pré-employé, syn. de Squalus L. 1758.

Centrophoroides Davis 1887.

Centrophorus Müller et Henle 1838: syn. de Dalatias R. 1810.

Centroscyllium Müller et Henle 1838.

Centroscymnus Bocage et Capello 1864.

Centroselachus GARMAN 1913.

Cerictius RAFINESQUE 1810: non valide.

Dalatias Rafinesque 1810.

Deania JORDAN et SNYDER 1902.

Deaniops WhItLEY 1932.

Entoxychirus GILL 1862: syn. de Dalatias R. 1810.

Etmopterus RAFINESQUE 1810.

Flakeus WhitLey 1939: s./g. de Squalus L. 1758.
Gaboa Whitley 1940 : s./g. de Dalatias R. 1810.

Koinga Whitley 1939: s./g. de Squalus L. 1758.

Lepidorhinus BONAPARTE 1845.

Machephilus JoHNSON 1867 : syn. de Lepidorhinus B. 1845.

Nasisqualus SMITH 1912.

Paracentroscyllium ALCock 1889.

Proscymnodon FowLER 1934 : s /g. de Scymnodon B. et C. 1864.

Pseudoscymnus Herre 1935.

Scymnodon Bocage et Capello 1864.

Somnisphinax : errat. pro Somnispinax W. 1940.

Somnispinax WhItLeY 1940: s./g. de Dalatias R. 1810.

Spinax CUVIER 1817 : syn. d'Etmopterus R. 1810.

Spinax Bonaparte 1845 : syn. d'Etmopterus R. 1810.

Squalus LINNÉ 1758.

Zameus JORDAN et FOWLER 1903 : syn. de Scynodon B. et C. 1864.

\section{FAMILLE DES OXYNOTIDAE}

(Humantin ou Cochon de mer)

Centrina Cuvier 1817 : syn. d'Oxynotus $R .1810$.

Oxynotus RAFINESQUE 1810.

$$
\begin{gathered}
\text { FAMILLE DES } \\
\text { SCYMNORHINIDAE } \\
\text { (= Echinorhinidae) } \\
\text { (Requins bouclés) }
\end{gathered}
$$

Borborodes Gistel 1848: syn. de Scymnorhinus B. 1845.

Cirrhigaleus TANAKA 1912.

Echinorhinus DE BLAINVILLE 1816.

Euprotomicrus GILL 1864.

Goniodus Agassiz 1836.

Isistius GILL 1864.

Leius KNER 1865 : syn. de Isitius G. 1864.

Phaenopogon Herre 1935: syn. de Cirrhigaleus T. 1912. 
Rubusqualus WHITLEY 1931: s.'g. d'Echinorhinus B. 1816.

Scimnus S. D. W. 1837: syn. de Scymnorhinus B. 1845.

Scymnodalatias GARRICK 1956. Scymnorhinus BONAPARTE 1845.

Scymnus CUVIER 1817 : pré-employé, syn. de Scymnorhinus B. 1845. Squaliolus SмIтH 1912.

\section{FAMILLE DES SOMNIOSIDAE}

(Dormeurs ; Sleeper-sharks)

Heteroscymnoides FOWLER 1934.

Heteroscymnus TANAKA 1912.

Laemargus Müller et Henle 1838 : syn. de Somniosus S. 1818.

Leiodon WooD 1847 : pré-employé, syn. de Somniosus S. 1818.

Rhinoscymnus GILL 1864.

Somniosus LE SUEUR 1818.

\section{FAMILLE DES \\ PRISTIOPHORIDAE}

(Saw-sharks)

Pristiophorus Müller et Henle 1837.

FAMILLE DES

PLIOTREMIDAE

Pliotrema REGAN 1906.

\section{Sous-ordre des SQUATINOIDEI}

\section{FAMILLE DES SQU.ATINIDAE}

(Anges de mer ; Angel-sharks)

Rhina KLEIN 1775 : non valide.

Rhina RAFINESQUe 1810: syn. de Squatina D. 1806.

Rhina (K.) GILl 1861 : syn. de Squatina D. 1806.

Squalraia DE LA PYLAIE 1835: syn. de Squatina D. 1806.

Squatina DUMÉRIL 1806.
SERIE DES HYPOTREMA

Ordre des RAJIFORMES Sous-ordre des RHINOBATOIDEI

FAMILLE DES RHINIDAE

(= Rhamphobatidae

= Rynchobatidae)

Demiurga Gistel 1848: syn. de Rhina B. et S. 1801.

Rhamphobatis GILL 1861: syn. de Rhina B. et S. 1801.

Rhina BLOCH et SCHNEIDER 1801.

Rhynchobatis: vide Rhynchobatus M. et H. 1837.

Rhynchobatus MülleR et HeNLE 1837.

FAMILLE DES RHINOBATOIDAE $(=$ Rhinobatidae $)$

(Guitares de mer; Guitar-fishes)

Acroteriobatus GiLtaY 1928: s./g. de Rhinobatos L. 1790.

Aplychotrema NoRMAN 1926.

Glaucostegus BONAPARTE 1845 : syn. de Rhinobatos L. 1790.

Leiobatus RAFINESQUE 1810 : syn. de Rhinobatos L. 1790.

Platypornax WhITLEY 1939: s./g. de Rhinobatos L. 1790.

Rhinobatos LINCK 1790.

Rhinobatus BLOCH et SCHNEIDER 1801: syn. de Rhinobatos L. 1790 .

Rhynchobatis PHILIPPI 1857: préemployé, syn. de Tarsites J. 1919.

Scobatus WhitLey 1939: s./g. de Rhinobatos L. 1790.

Squatinoraja NARDO 1824: syn. de Rhinobatos L. 1790.

Syrrhina Müller et Henle 1838 : syn. de Rhinobatos L. 1790.

Tarsites JORDAN 1919.

Trygonorhina MüLleR et HENLE 1841.

Trygonorrhina MüLLER et HENLE 1838.

Zapteryx Jordan et GILBERT 1880. 
FAMILLE DES PLATYRHINIDAE $(=$ Discobatidae $=$ Analithidae $)$

Analithis Gistel 1848 : syn. de Platyrhina M. et H. 1838.

Discobatus Garman 1880: syn. de Platyrhina M. et H. 1838.

Platyrhina Müller et Henle 1838: parfs. considéré comme pré-employé et remplacé par Analithis G. 1848 .

Platyrhinoidis GARMAN 1880.

\section{FAMILLE DES PRISTIDAE}

(Poissons-scies ; Saw-fishes)

Ctenopristis ARAmbourg 1941.

Myriosteon GraY 1864: syn. de Pristis L. 1790.

Pristibatis SeRVILLe 1829.

Pristiopsis FOWLER 1905.

Pristis KLeIN 1775 : non valide.

Pristis LINCK 1790.

Pristis Latham 1794 : syn. de Pristis L. 1790.

Schizorhiza WeILER 1930.

\section{Sous-ordre des RAJOIDEI}

\section{FAMILLE DES RAJIDAE}

(Raies et Pocheteaux ; Rays and Skates)

Amblyraja Malm 1877.

Arctoraja Ishiyama $1958:$ s./g. de Breviraja B. et S. 1948.

Argoraja Whitley 1940: s./g. de Raja L. 1758.

Arthrobatis WhITLEY 1940.

Arthropterus AgASSIZ 1840 : pré-employé, syn. d'Arthrobatis W. 1940.

Bathyraja IsHIYAMA 1958: s./g. de Breviraja B. et S. 1948.

Batis BONAPARTE 1837 : syn. de Raja L. 1758.

Breviraja BIgELOW et SCHROEDER 1948.

Cephaleutherus RAFINESQUE 1810 : syn. de Raja L. 1758.
Cruriraja Bigelow et SCHROEder 1948.

Dactylobatus BEAN et WEED 1909. Dasybatis Serville 1829: syn. de Raja L. 1758.

Dasybatus BONAPARTE 1837 : syn. de Raja L. 1758.

Deitiraja WhITLEY 1940 : s./g. de Raja L. 1758.

Dipturus RAFINESQUe 1810: syn. de Raja L. 1758.

Hieroptera Fleming 1841: syn. de Raja L. 1758.

Irolita WHITLEY 1931.

Lacviraja BONAPARTE 1837.

Laevirajae NARDO 1827: non valide. Leiobatus KLEIN 1775 : non valide.

Leucoraja MALM 1877.

Malacobatis Gracianov 1907.

Malacorhina GARMAN 1877.

Notoraja Ishryama 1958: s./g. de Breviraja B. et S. 1948.

Okamejei Ishiyama 1958: s./g. de Raja L. 1758.

Parvoraja Whitley 1939: s./g. de Raja L. 1758.

Pla:opterus RAFINESQUe 1815 : syn. de Raja L. 1758.

Propterygia OTTO 1824.

Psammobatis GüNTHER 1870.

Psainmobatus: vide Psammobatis G. 1870.

Raia: emend. pro Raja L. 1758.

Raja LINNÉ 1758.

Rajabatis DE LA PYLAIE 1835: non valide.

Rhinoraja IshIYAMA 1952.

Rioraja WhItLey 1939: s./g. de Raja L. 1758.

Spi.uiraja WhITLEY 1939: s./g. de Raja L. 1758.

Synipterygia Müller et Henle 1838 .

Tengujei IshiYama 1958: s./g. de Raja L. 1758.

Uraptera Müller et Henle 1838.

Zearaja WhITLEY 1939.

FAM. DES PSEUDORAJIDAE

Pseudoraja Bigelow et Schroeder 1954. 
FAM. DES ARHYNCHOBATIDAE Arhynchobatis WAITE 1909.

\section{Sous-ordre des DASYATOIDEI}

FAMILLE DES DASYATIDAE $(=$ Trygonidae $=$ Dasybatidae $)$ (Pastenagues; Sting-rays)

Amphotistius Garman 1913.

Anacanthobatis BONDE et SWART 1924.

Anacanthus (EHRENBERG) CUVIER 1829: pré-employé, syn. d'Urogymnus M. et H. 1837.

Bathytoshia WhItLEY 1933.

Dasyatis RAFINESQUE 1810.

Dasybatus KLEIN 1775 : non valide.

Dasybatus (K.) Garman 1885: syn. de Dasyatis R. 1810.

Dasybatus (K. ; Walbaum) Ogilby 1916: syn. de Dasyatis R. 1810.

Discobatis MiKLUKHo-MACLEAY et MACLEAY 1886: pré-employé, syn. de Taeniura M. et H. 1837.

Discotrygon Fowler 1910: syn. de Taeniura M. et H. 1837.

Gymnura MüLleR et Henle 1837: syn. d'Urogymnus $M$. et $\mathrm{H}$. 1837.

Hemitrygon Müller et Henle 1837.

Himantura MülLER et HeNle 1837: syn. d'Urogymnus $M$. et $\mathrm{H}$. 1837.

Hyplophus MüLler et Henle 1838 : errat. pro Hypolophus M. et H. 1838.

Hypolophus Müller et Henle 1838 : syn. de Pastinachus R. 1828.

Leiobatus DE BlaINVILle 1816: préemployé, syn. d'Urotrygon G. 1864.

Neotrygon CASTELnaU 1873.

Pastinaca Dekay 1842: syn. de Dasyatis R. 1810.

Pastinaca Gronow 1854 : syn. de Dasyatis R. 1810.

Pastinacae NARDO 1827 : non valide.

Pastinachus RüPPEll 1828.

Pteroplatea Müller et Henle 1838. Pteroplatytrygon FOWLER 1910.
Rhachinotus CANTOR 1849: syn. d'Urogymnus M. et H. 1837. Springeria BIGELOW et SCHROEdeR 1951.

Taeniura MüLler et Henle 1837.

Toshia WhITLEy 1933.

Trygon (AdANSON) CUVIER 1817 : syn. de Dasyatis R. 1810.

Trygon Is. GeoffroY - ST - Hilaire 1826: syn. de Taeniura M. et H. $183 \%$.

Trygonobatus DE BLAINVILLE 1816: syn. de Dasyatis R. 1810.

Trygonoptera MülLeR et Henle 1838.

Urobatis GARMAN 1913.

Urogymnus Müller et Henle 1837.

Urolophoides SOLDATOV et LiNDBERG 1930.

Urolophus Müller et Henle 1838. Urotrygon GILL 1863.

Uroxis RAFINESQUE 1810: syn. de Dasyatis R. 1810.

FAMILLE DES

POTAMOTRYGONIDAE

(= Elipesuridae)

Disceus Garman 1877 : syn. de Paratrygon D. 1865.

Elipesurus ScHOMBURGK 1842.

Paratrygon DUMÉRIL 1865.

Potamotrygon GARMAN 1877.

\section{FAM. DES BRACHIOPTERIDAE}

Brachioptera GRACIANOV 1906.

Brachyoptera : vide Brachioptera G. 1906.

Phanerocephalus Gracianov 1906. Planerocephalus GRACIANOV 1906: errat. pro Phanerocephalus G. 1906.

FAMILLE DES AETOBATIDAE (= Myliobatidae)

(Aigles de mer; Eagle-rays)

Aetobatis: vide Aetobatus B. 1816 .

Aetobatus de Blainville 1816.

Aetomylaeus Garman 1908. 
Aetoplatea Müller et Henle 1838. Goniobatis Agassiz 1858: syn. d'Aetobatus B. 1816.

Holorhinus GILL 1862.

Indomanta WHITLEY 1926.

Myliobatis (DUMÉRIL) CUVIER 1817: syn. d'Aetobatus B. 1816.

Pteromylaeus GARMAN 1913.

Stoasodon CANTOR 1849.

FAMILLE DES RHINOPTERIDAE

Micromesus GILL 1865.

Mylorhina GILL 1865.

Rhinoptera (KUHL) CUVIER 1829.

Zygobatis Agassiz 1836: syn. de Rhinoptera (K.) C. 1829.

\section{FAMILLE DES MOBULIDAE}

( $=$ Mantidae $=$ Cephalopteridae $)$

(Diables de mer ou raies cornues; Devil-rays)

Apterurus RAFINESQUe 1810: syn. de Mobula R. 1810.

Brachioptilon NeUman 1849: syn. de Manta B. 1829.

Cephaloptera Fleming 1822: syn. de Mobula R. 1810.

Cephalopterus Risso 1810: pré-employé, syn. de Mobula R. 1810.

Ceratobatis BOULENGER 1897.

Ceratoptera Müller et Henle 1838: syn. de Manta B. 1829.

Daemomanta WHITLEY 1944.

Diabolichthys Holmes 1856: syn. de Manta B. 1829.

Dicerobatus DE Blainville 1816: syn. de Mobula R. 1810.

Manta BANCROFT 1829.

Mobula RAFINESQUE 1810.

Pterocephala SwaInson 1839: syn. de Mobula R. 1810.

\section{Ordre des TORPEDINIFORMES}

\section{FAMILLE DES TORPEDINIDAE} (= Narcobatidae = Narcaciontidae)

(Torpilles ; Numb-fish)
Astrape Müller et Henle 1838: syn. de Narke K. 1826.

Bengalichthys ANNANDALE 1909.

Benthobatis AlcocK 1898.

Crassinarke TAGAKI 1951.

Cyclonarce GILL 1861 : syn. de Narcine $\mathrm{H} .1834$.

Diplobatis BIgELOW et SCHROEDER 1948.

Discopyge Tschudi 1846.

Eunarce FOWLER 1910.

Fimbriotorpedo FRISTCH 1886: syn. de Torpedo D. 1806.

Gonionarce GILL 1861 : syn. de Narcine H. 1834.

Gymnotorpedo FRITSCH 1886.

Heteronarce REGAN 1921.

Hypnarce WAITE 1902.

Hypnos DUMÉRIL 1852 : pré-employé, syn. d'Hypnarce W. 1902.

Narcacion KLEIN 1775: non valide, syn. de Torpedo D. 1806.

Narcacion (K.) BLEEKER 1866: syn. de Torpedo D. 1806.

Narcine HENLE 1834.

Narcinops WhItLey 1940.

Narcobatis (B.) SeRville 1830 : syn. de Torpedo D. 1806.

Narcobatus DE BLAINVILLE 1816 : syn. de Torpedo D. 1806.

Narke KaUP 1826.

Notastrape WhITLEY 1932.

Syrraxis (JouRdAN) BONAPARTE 1841 : syn. de Narcine H. 1834.

Temera GRAY 1831.

Tetranarce GILL 1861: s./g. de Torpedo D. 1806.

Tetronarce GILL 1861: errat. pro Tetranarce G. 1861.

Tetronarcine TANAKA 1908.

Torpedo FORSKAL 1775: non valide.

Torpedo HoutTuYN 1764: non valide.

Torpedo DUMÉRIL 1806.

Torpedo RAFINESQUE 1810: syn. de Torpedo D. 1806.

Typhlonarke WAITE 1909. 


\section{Sous-classe des Bradyodontes}

\section{(Bradyodontia)}

\section{Ordre des HOLOCEPHALES}

FAMILLE DES CHIMAERIDAE (Chimères ; Elephant-fishes)

Bathyalopex Collett 1901.

Chimaera LINNÉ 1758.

Hydrolagus GILL 1862.

Phasmichthys JORDAN et HUBBS 1925.

Psychichthys FOWLER 1907.

FAM. DES RHINOCHIMAERIDAE Anteliochimaera TANAKA 1909.
Antillochimaera: errat. pro Anteliochimaera T. 1909.

Harriotta GoOde et BEAN 1894.

Neoharriotta BIGELOW et SCHROEDER 1950.

Rhinochimaera GARMAN 1901.

FAM. DES CALLORHYNCHIDAE Callorhynchus Gronow 1763: non valide.

Callorhynchus (G.) CUVIER 1817.

\section{Osteichtbyes}

\section{Sous-classe des Actinopterygiens}

\section{(Actinopterygia)}

CHONDROSTEI.

Ordre des ACIPENSERIFORMES

FAMILLE DES ACIPENSERIDAE (Esturgeons ; Sturgeons)

Acipenser LinNÉ 1758.

Antaceus Heckel et Fitzinger 1836.

Dinectus RAFINESQUE 1818: non valide.

Ellops Gistel 1848: emend. pro Helops B. et R. 1833.
Helops Brandt et Ratzeburg 1833. Hemiscapirhynchus BERG 1912.

Huso BrandT et RATZEBurg 1833: syn. d'Ichthyocolla G.-ST-H. 1767.

Ichthyocolla GeOFFroY-ST-HILAIRE 1767.

Kessleria Boghdanov 1882: pré-employé, syn. de Pseudoscapirhynchus N. 1900.

Lioniscus Heckel et FITZINGER 1836.

Parascapirhynchus ForBes et RICHARDSON 1905. 


\section{Pseudoscapirhynchus NIKOLSKY} 1900.

Scaphirhynchops GILL 1863.

Scaphirhynchus HECKEL 1836: syn. de Scaphirhynchops G. 1863.

Scapirhynchus MüLLER 1835.

Schipa BRANDT 1869: syn. d'Antaceus H. et F. 1836.

Sinosturio JAECKEL 1929.

Sterledus BonAPARTE 1845: syn. de Ichthyocolla G.-ST-H. 1767.

Sterleta GüldENSTADT 1772: syn. de Ichthyocolla G.-ST-H. 1767.

Sterletus RAFINESQUE 1820: syn. de Ichthyocolla G.-ST-H. 1767.

Sterletus BRANDT et RATZEBURG 1833 : syn. de Ichthyocolla G.-ST-H. 1767.

Sturio RAFINESQUe 1810.

Sturio Müller 1835 : syn. d'Acipenser L. 1758.

FAMILLE DES POLYODONTIDAE

(Bec-en-cuillère ; Paddle-fishes)

Platirostra Lesueur 1818: syn. de Polyodon L. 1797.

Polyodon DE LACÉPÈDE 1797.

Polyodon BLOCH et SCHNEIDER 1801 : syn. de Polyodon L. 1797.

Proceros RAFINESQUE 1820: non valide.

Psephurus GÜNTHER 1873.

Spatularia SHaw 1804: syn. de Polyodon L. 1797.

HOLOSTEI.

Ordre des AMIIFORMES

FAMILLE DES AMIIDAE

$$
\text { (= Amiatidae) }
$$

(Amies ; Bow-fish, Dog-fisk or Mud-fish)

Amia LINNÉ 1766.

Amiatus RAFINESQUe 1815: syn. d'Amia L. 1766.
FAMILLE DES LEPISOSTEIDAE

(Brochets-lances ; Gar-pikes) Acus CATEsBy 1771: non valide. Atractosteus RAFINESQUE 1820. Cylindrosteus RAFINESQUE 1820. Lepidosteus KöNIG 1825: syn. de Lepisosteus L. 1803.

Lepisosteus DE LACÉPÈDE 1803.

Litholepis RAFINESQUE 1818: non valide.

Psalisostomus KLEIN 1781: non valide.

Sarchirus RAFINESQUE 1818: juvénile de Lepisosteus L. 1803.

\section{TELEOSTEI.}

Ordre des CLUPEIFORMES

\section{Sous-ordre des ELOPOIDEI}

\section{FAMILLE DES ELOPOIDEI}

(Ten-pounder)

Elops LINNÉ 1766.

Gularus WHITLEY 1940 : s./g. d'Elops L. 1766.

Mugilomorus DE LACÉPÈDE 1803 : syn. d'Elops L. 1766.

Trichonotus RAFINESQUE 1815: syn. d'Elops L. 1766.

FAMILLE DES MEGALOPIDAE (Grande-écaille, Tarpon)

Amia Browne 1789: non valide.

Brisbania CASTELNAU 1877.

Megalops DE LACÉPÈDE 1803.

Oculeus (COMmerson) DE LACÉPÈdE 1803: non valide.

Tarpon JORDAN et EVERMANN 1896.

\section{FAMILLE DES ALBULIDAE}

(Banane de mer; Lady-fish) Albula Gronov 1763: non valide. Albula Scopoli 1777.

Albula Bloch et SCHNEIDER 1801: syn. d'Albula S. 1777.

Butyrinus DE LACÉPÈDE 1803: syn. d'Albula S. 1777. 
Conorhynchus (Nozeman) GILl 1861: syn. d'Albula S. 1777.

Dixonina FOWLER 1911.

Glossodus Cuvier 1815: syn. d'Albula S. 1777.

FAM. DES PTEROTHRISSIDAE (= Bathythrissidae)

Bathythrissa GüNTHER 1877 : syn. de Pterothrissus H. 1877.

Pterothrissus HILgendorf 1877.

Sous-ordre des NOTOPTEROIDEI

\section{FAMILLE DES HIODONTIDAE (Moon-fish)}

Amphiodon RAFINESQUe 1819.

Clodalus RAFINESQUE 1820: syn. d'Hiodon S. 1818.

Elattonistius GILL et JoRDAN 1877 : syn. d'Amphiodon R. 1819.

Glossodon RAFINESQUE 1818: syn. d'Hiodon S. 1818.

Glossodon Heckel. 1842 : syn. d'Hiodon S. 1818.

Hiodon Lesueur 1818.

Hyodon RAFINESQUE 1820: syn. d'Hiodon S. 1818.

FAMILLE DES NOTOPTERIDAE

Chitala Fowler 1934: s./g. de Notopterus L. 1800.

Glanis Gronow 1854: syn. de Notopterus L. 1800.

Notopterus de LACÉPÈdE 1800.

Xenomystus GÜNTHER 1868.

Sous-ordre des ALEPOCEPHALOIDEI

FAM. DES ALEPOCEPHALIDAE Alcockella FOWLeR 1934: s./g. de Narcetes A. 1890.

Alepocephalus Risso 1820.
Aleposomus GILl 1884: syn. de Xenodermichthys G. 1878.

Aleposomus Roule 19.

Anomalopterichthys WHITLEY 1940. Anomalopterus Vaillant 1886: préemployé, syn. d'Anomalopterichthys W. 1940.

Asquamiceps ZugMAYER 1911.

Auchenalepoceps FOWLER 1943: s./g. de Xenodermichthys G. 1878.

Aulostomatomorpha ALCOCK 1890.

Bajacalifornia TOWNSEND et NICHOLS 1925.

Bathypropteron FOWLER 1934: s./g. d'Aleposomus R. 19

Bathytroctes GüNTHER 1878.

Bellocia PARR 1951.

Benthosphyraena COCKERELL 1919. Binghamia PARR 1937: pré-employé, syn. de Binghamichthys W. 1941.

Binghamichthys WHITLEY 1941.

Brunichthys PARR 1951.

Caudania Roule 1935.

Conocara Goode et BeAn 1895.

Dolichopteryx BRAUER 1901.

Einara PARR 1951.

Ericara GILl et TownSEND 1897.

Esunculus KaUP 1856: larve d'Ale. pocephalidae $\mathrm{sp}$.

Grimatroctes PARR 1952.

Halisauriceps FOWLER 1934.

Lepogenys PARR 1951.

Leptochilichthys GARMAN 1899.

Leptoderma VAILlant 1886.

Lloydiella PARR 1952: s./g. d'Alepocephalus $\mathrm{R} .1820$.

Macromastax BEEBE 1933.

Megalepocephalus FoWLER 1934: s./g. d'Asquamiceps Z. 1911.

Mirognathus PARR 1951.

Mirorictus PARR 1947.

Mitchillina JoRDAN et EvERMANN 1896.

Narcetes Alcock 1890. 
Nemabathytroctes FOWLER 1934.

Nomoctes PARR 1952: s./g. de Bathytroctes.

Noriona Strand 1950: syn. de Proditor W. 1940.

Normania PARR 1937: syn. de Proditor W. 1940.

Perioceps PARR 1954: s./g. d'Asquamiceps Z. 1911.

Photostylus BeEBE 1933.

Platytroctegen LLOYD 1937.

Platytroctes GüNTHER 1878.

Pomatias Bloch et SchNeIDER 1801 : syn. de Triurus L. 1800.

Proditor WhITLEy 1940.

Rinoctes PARR 1952.

Rouleina JORDAN 1923: syn. d'Aleposomus Roule.

Talismania GoODE et BEAN 1895.

Tauredophidium ALCOCK 1890.

Torictus PARR 1951.

Triurus DE LACÉPÈDE 1800.

Whitleyidea FOWLER 1934: s./g. d'Alepocephalus R. 1820.

Xenodermichthys GüNTHER 1878.

Xenognathus GILBERT 1915: syn. d'Ericara G. et T. 1897.

\section{FAMILLE DES MACRISTIIDAE}

Macristium REGAN 1903.

\section{Sous-ordre des CHANOIDEI}

FAMILLE DES KNERIIDAE

Angola MYERS 1928.

Kneria SteIndachner 1866.

Xenopomatichthys BOULENGER 1910.

\section{FAMILLE DES CHANIDAE}

Chanos DE LACÉPÈdE 1803.

Lutodeira RüPPELL 1828: syn. de Chanos L. 1803.
Ptycholepis GraY 1842: syn. de Chanos L. 1803.

Scoliostomus RüPPELL 1828 : syn. de Chanos L. 1803.

FAMILLE DES

ANCYLOSTYLIDAE

Ancylostylos Kramberger 1895.

\section{FAMILLE DES \\ PHRACTOLAEMIDAE}

Phractolaemus BOULENGER 1901.

Sous-ordre des CLUPEOIDEI

FAMILLE DES SEARSIDAE

Barbantus PARR 1951.

Holtbyrnia PARR 1937.

Mentodus PARR 1951: s./g. de Holtbyrnia P. 1937.

Normichthys PARR 1951.

Pellisolus PARR 1951.

Persparsia PARR 1951.

Sagamichthys PARR 1953.

Searsia PARR 1937.

\section{FAMILLE DES \\ CHIROCENTRIDAE}

Chirocentrus CUVIER 1817.

Neosudis CASTElnau 1873: syn. de Chirocentrus C. 1817.

\section{FAMILLE DES CLUPEIDAE}

(Hareng, Sardine, Alose, etc. ;

$$
\text { Herring) }
$$

Alausa Cuvier et VAlenCIENNES 1847: syn. d'Alosa L. 1790.

Alausella GILL 1861: syn. de Pomolobus R. 1820.

Alosa LINCK 1790.

Alosa Cuvier 1829: syn. d'Alosa L. 1790.

Amblygaster BleEKer 1849: syn. de Sardinella C. et V. 1847. 
Amentum Whitley 1940.

Antu De Buen 1959: s./g. de Clupea L. 1758.

Apterogasterus DE LA PYLAIE 1835. Apterygia Gray 1833: syn. de Raconda G. 1831.

Arengus: syn. de Harengus G.-ST-H. 1767.

Brevoortia GILL 1861.

Caspialosa BeRg 1915.

Chirocentrodon GüNTHER 1868.

Clupalosa BLEEKER 1849.

Clupea LINNÉ 1758.

Clupeichthys BLEEKER 1855 : syn. de Corica H. 1822.

Clupeoides BLEEKER 1851: syn. de Corica H. 1822.

Clupeonella KESSLER 1877.

Clupeonella Berg 1913 : syn. de Caspialosa B. 1915.

Clupeonia Cuvier et VAlenciennes 1847.

Corica Hamilton 1822.

Cynothrissa RegaN 1917.

Dascillus Gronov 1854: non valide (Clupéide mutilé).

Dastilbe JoRdan 1910.

Ehirava Derantyagala 1929.

Escualosa WhItLEY 1940.

Ethmalosa Regan 1916.

Ethmidium THOMPSON 1916.

Euplatygaster FOWLER 1934: s./g. d'Ilisha G. 1845.

Filialosa FowLER 1944.

Fimbriclupea WHITLEY 1940.

Fiscinia WhItLeY 1940.

Fluvialosa WHITLEY 1943.

Gnathobolus BLOCH et SCHNEIDER 1801: syn. de Odontognathus L. 1800 .

Gudusia FOWLER 1911.

Hallecula JoRDAN 1925 : pré-employé, syn. de Parahalecula F. 1959.

Harengula CUVIER et VALENCIENNES 1847.

Harengus Geoffroy-St-Hilaire 1767.
Harengus CATESBY 1771: non valide. Harengus KLEIN 1775: non valide. Heringia FOWLER 1911.

Herklotsella FOWLER 1934: pré-employé, syn. de Herklotsichthys W. 1953.

Herklotsichthys WHITLEY 1953 : s./g. d'Harengula C. et V. 1847.

Hildebrandichthys SCHULze 1950.

Hilsa Regan 1916: syn. de Harengula C. et V. 1847.

Hyperlophus OgILBy 1892.

Hyrtlinus FOWLER 1959.

Ilisha GRAY 1845.

Kowala Cuvier et VAlenciennes 1847.

Lile Jordan et EVERMANN 1896.

Limnothrissa REGAN 1917.

Macrura VAN HASSELT 1823: préemployé, syn. de Harengula C. et V. 1847.

Maugeclupea WhitLey 1932.

Medipellona JoRDAN et SEALE 1926: syn. de Chirocentrodon G. 1868.

Meletta Cuvier et VALENCIENNES 1847 : syn. de Spratella C. et V. 1847 .

Microthrissa BOULENGER 1902.

Neoopisthopterus HILDEBRAND 1948.

Neosteus NoRMAN 1923.

Nilotica : s./g. de Harengula C. et V. 1847.

Odaxothrissa BOULENGER 1899.

Odontognathus DE LACÉPÈDE 1800.

Omochetus OGIBY 1897: s./g. d'Hyperlophus O. 1892.

Opisotomus (COMMERSON) DE LACÉPÈDE 1803: non valide.

Opisthonema GILL 1861.

Opisthopterus GILL 1861.

Parahalecula FOWLER 1959.

Paralosa BLEEKER 1872: non valide.

Paralosa REgAN 1916: syn. de Harengula $\mathrm{C}$. et V. 1847.

Pellona CUVIER et VALENCIENNES 1847: syn. de Ilisha G. 1845. 
Pellonula GÜNTHER 1868.

Pellonulops SMITH 1949.

Platygaster SwaINSON 1839: syn. d'Ilisha G. 1845.

Pliosteostoma Norman 1923.

Poecilothrissa Regan 1917.

Pomatias BLOCH et SCHNEIDER 1801.

Pomolobus RAFInesQue 1820.

Potamalosa OgILby 1896.

Potamothrissa RegAN 1917: s./g. de Microthrissa B. 1902.

Pristigaster CuvIer 1817: syn. de Ilisha G. 1846.

Pseudochirocentrodon RIBEIRO 1922.

Raconda GRAY 1831.

Rhinosardinia EIGENMANN 1912 : syn. de Heringia F. 1911.

Rogenia Cuvier et VAlenciennes 1847: juvénile de Clupea L. 1758.

Sardina ANTIPA 1905: syn. de Sardinia POEY 1860.

Sardinella CuVIER et VALENCIENNES 1847.

Sardinia PoEY 1869: valide fide JORDAN 1919.

Sasdinops Hubbs 1929.

Sauvagella BERTIN 1943: syn. de Spratellomorpha A., B. et G. 1945.

Spratella Cuvier et VALenciennes 1847.

Spratellomorpha ANGEL, BERTIN et GuiBÉ 1945.

Sprattus GIRgensohn 1846.

Stolothrissa REGAN 1917.

Tenualosa Fowler 1934: s./g. de Harengula C. et V. 1847.

Trichis (PlUMIER) DE LACÉPÈDE 1803: non valide.

Wilkesina FOWLER et BEAN 1923 : s./g. de Harengula C. et V. 1847.

Zunasia Jordan et METZ 1913 : syn. de Ilisha G. 1845.

\author{
FAMILLE DES \\ DUSSUMIERIDAE \\ (Round-herring)
}

Dussumieria CUVier et VALENCIENNES 1847.

Eutrumeus BI.EEKER 1853.

Gilchristella FOWLER 1935.

Jenkinsia JORDAN et EVERMANN 1896.

Leptogaster BLEEKER 1872: non valide, syn. de Spratelloides L. 1803.

Montalbania FowLER 1934: s./g. d'Eutrumeus B. 1853.

Perkinsia EIgenmann 1891.

Sauvagella BERTIN 1940 : syn. de Gilchristeila F. 1935.

Smithites JORDAN et GILBERT 1912.

Spratelloides DE LACÉPÈDE 1803.

Spratelloides BLEEKER 1851: syn. de Spratelloides L. 1803.

Stolephorus DE LACÉPÈDE 1803 : transféré aux Engraulidae s.s., l'espèce type étant un Anchois.

FAMILLE DES DOROSOMIDAE (Gizzard-shad)

Anodontostoma BLEEKER 1849.

Chatoessus Cuvier 1829: syn. de Dorosoma R. 1820.

Clupanodon DE LACÉPÈDE 1803.

Dorosoma RAFINESQUE 1820.

Gonialosa REgAN 1916.

Gonostoma van HASSEI.T 1823 : préemployé, syn. de Anodontostoma B. 1849.

Indialosa Herre et MYERS 1931: s./g. de Gonialosa R. 1916.

Konosirus Jordan et SNYDER 1900: syn. de Clupanodon L. 1803.

Nealosa MYers et HubBs 1931 : s./g. de Clupanodon L. 1803.

Nematalosa REgaN 1916.

Signalosa EvermanN et Kendall 1898.

Thrissa RAFINESQUE 1815: syn. de Clupanodon L. 1803. 


\section{FAMILLE DES ENGRAULIDAE} (Anchois, Anchovy)

Amplova Jordan et Seale 1925.

Amplova Whitley 1940 : s./g. d'Anchoviella F. 1911.

Anchoa Jordan et Evermann 1927 : s./g. d'Engraulis C. 1817.

Anchovia Jordan et Evermann 1895. Anchoviella FOWLER 1911.

Austranchovia WhItLey 1931.

Cetengraulis GüNTHER 1868.

Chaetomus Mc Clelland 1844.

Coilia Gray 1831.

Copeichthys Dollo 1904.

Demicoilia JoRdan et SEAle 1925.

Diplomystus Cope 1877 : pré-employé, syn. de Copeichthys D. 1904.

Encrasicholina FOWLER 1938.

Encrasicholus (COMMERSON) DE LACÉPÈDE 1803: non valide.

Encrasicholus FLEMING 1828: syn. d'Engraulis C. 1817.

Engraulis CUVIER 1817.

Heterothrissa GüNTHER 1868.

Leptonurus BLEEKER 1849: non valide, syn. de Coilia G. 1831.

Lycengraulis GüNTHER 1868.

Lycothrissa GüNTHER 1868.

Menidia BROWNE 1789: non valide. Mystus DE LACÉPÈDE 1803 : pré-employé, syn. de Coilia G. 1831.

Osteoglossum BASILEWSKI 1855 : préemployé, syn. de Chaetomus MC. 1844.

Pterengraulis GüNTHER 1868.

Scutengraulis JoRDAN et SEALE 1925.

Setipinna SwaINSON 1839.

Stethochaetus Gronow 1854 : syn. de Setipinna S. 1839.

Stolephorus DE LACÉPÈDE 1803.

Telara GüNTHER 1868: syn. de Setipinna S. 1839.

Thrissa Cuvier 1817: pré-employé, syn. de Thrissocles J. 1917.

Thrissina JoRdan et SEALE 1925.
Thrissocles JORDAN 1917.

Thryssa: vide Thrissa C. 1817.

Trichosoma SWAINSON 1839.

Xenengraulis JoRdan et Seale 1925.

\section{Sous-ordre des CROMEROIDEI}

FAMILLE DES CROMERIIDAE

Cromeria Boulenger 1901.

FAMILLE DES DENTICIPITIDAE

Denticeps Clausen 1959.

Igborichthys Clausen 1959: syn. de Denticeps C. 1959.

\section{Sous-ordre des SALMONOIDEI}

\section{FAMILLE DES SALMONIDAE}

(Saumons et Truites, Salmons and Trouts)

Baione DeKay 1842: syn. de Salvelinus (N.) R. 1836.

Cristivomer GILL et JoRdan 1878. Epitomynis Schulze 1889: syn. de

Hucho G. pp. et de Salvelinus R. pp.

Fario CuVIER et VAlenciennes 1848: syn. de Trutta G.-ST-H. 1768.

Hucho GüNTHER 1866.

Hypsifario GILL 1862.

Oncorhynchus SUCKLEY 1860.

Phyllogephyra BOULENGER 1898.

Salar CUVIER et VALENCIENNES 1848: syn. de Salmo L. 1758.

Salmo LINNÉ 1758.

Salvelini NILsson 1832: non valide. Salvelinus RICHARDSON 1836.

Trutta Geoffroy-ST-HilaIRE 1767 :

s./g. de Salmo L. 1758.

Trutta KLEIN 1775: non valide.

Truttae LINNÉ 1758: non valide.

Umbla Rapp 1854: syn. de Salvelinus (N.) R. 1836. 
FAMILLE DES PLECOGLOSSIDAE (Ayu)

Plecoglossus Temminck et Schlegel 1846.

\section{FAMILLE DES COREGONIDAE}

(Corégones ou Lavarets; White-fishes or Ciscoes)

Allosomus JORDAN 1878.

Argyrosomus AgAssiz 1850 : pré-employé, syn. de Leucichthys D. 1874.

Brachymystax GüNTHER 1866.

Cisco Jordan et Evermann 1911.

Coregoni LINNÉ 1758: non valide.

Coregonus dE LACÉPÈdE 1803.

Irillion JORDAN 1919.

Leucichthys DYBOWSKI 1874.

Luciotrutta GüNTHÊR 1866: syn. de Stenodus R. 1836.

Prosopium MILNER 1878.

Stenodus RICHARDSON 1836.

Thrissomimus (GILL) JORDAN et EVERMANN 1911: syn. ou s./g. de Leucichthys D. 1874.

Tripteronotus DE LACÉPÈDE 1803 : syn. de Coregonus L. 1803.

\section{FAMILLE DES THYMALLIDAE (Ombre ; Grayling)}

Choregon Minding 1832: syn. de Thymallus L. 1790.

Orthocolus GISTEL 1848: syn. de Thymallus L. 1790.

Salmothymus BERG 1908.

Thymalloides BERG 1908.

Thymallus LINCK 1790.

Thymallus Cuvier 1829: syn. de Thymallus L. 1790.

\section{FAMILLE DES OSMERIDAE}

(Eperlans ou Prêtres; Smelt)

Allosmerus HuBss 1925.

Eperlanio JORDAN 1919.

Eperlanus GaImard 1849: syn. d'Osmerus L. 1803.

Hypomesus GILL 1862.
Mallotus CUVIER 1829.

Mesopus GILL 1862 : non valide, syn. d'Hypomesus C. 1829.

Osmeri LINNÉ 1758: non valide.

Osmerus DE LACÉPÈDE 1803.

Spirinchus JORDAN et EVERMANN 1896.

Thaleichthys GIRARD 1859.

Therobromus LUCAS 1898: serait syn. de Bathylagus G. 1878 (Bathylagidae).

\section{FAMILLE DES SALANGIDAE (Chinese White-bait)}

Albula OsBeck 1762: non valide, syn. de Salanx C. 1817.

Eperlanus BASILEWSKI 1855 : syn. de Salanx C. 1817.

Hemisalanx REgAN 1908.

Leucosoma Gray 1831: syn. de Sa$\operatorname{lanx}$ C. 1817.

Neosalanx WAKIYAWA et TAKAHASHI 1938.

Paraprotosalanx FANG 1934.

Parasalanx REgAN 1908.

Reganisalanx FANG 1934.

Salangichthys BLEEKER 1860.

Salanx CUVIER 1817.

\section{Sous-ordre des \\ OPISTHOPROCTOIDEI}

\section{FAMILLE DES \\ OPISTHOPROCTID.AE}

Grimaldia CHAPMAN 1942: pré-employé, syn. de Monacoa W. 1943.

Macropinna CHAPMAN 1939.

Monacoa WhITLEY 1943.

Opisthoproctus VAILLANT 1888.

Winteria BRAUER 1901.

FAMILLE DES ARGENTINIDAE (Argentins)

Acantholepis KRÖYER 1846: syn. d'Argentina L. 1758. 
Argentina LINNÉ 1758.

Bathylynchops COHEN 1958.

Bathymacrops GILCHRIST 1923.

Glossanodon GUICHENOT 1866.

Hyalorhynchus GILCHRIST et BONDE 1924 : pré-employé, syn. de Rhynchohyalus B. 1925.

Leuroglossus GILBERT 1890.

Ophthalmopelton MAUL: syn. de Rhyncholagus B.

Prosoarchus CoHen 1958: s./g. de Glossanodon G. 1866.

Rhynchohyalus BARNARD 1925.

Rhyncholagus BARNARD 1925.

Silus REINHARDT 1833: syn. d'Argentina L. 1758.

\section{FAMILLE DES MICROSTOMIDAE}

Euproserpa FOWLER 1934 : s./g. de Microstoma C. 1817.

Halaphya GÜNTHER 1889.

Microstoma CUVIER 1817.

Nansenia Jordan et EVERMANN 1896.

Xenophthalmichthys REGAN 1925.

FAMILLE DES BATHYLAGIDAE

Bathylagoides WHITLY 1951: s./g. de Bathylagus G. 1878.

Bathylagus GÜNTHER 1878.

Bathymacrops GILCHRIST 1922.

Therobromus Lucas 1898: syn. de Bathylagus G. 1878.

FAMILLE DES RETROPINNIDAE (= Retropinnatidae $)$

(New-Zealand White-bait)

Jenynsella OGILby 1908: syn. de Retropinna G. 1862.

Retropinna GILL 1862.

Richardsonia STEINDACHNER 1866: syn. de Retropinna G. 1862.

Stokellia WHITLEY 1955.

\section{Sous-ordre des GALAXIOIDEI}

\section{FAMILLE DES GALAXIIDAE}

Agalaxias Scotт 1935: s./g. de Galaxias C. 1817.

Austrocobitis OgILby 1899: syn. de Galaxias C. 1817.

Brachygalaxias EIGENMANN 1928.

Galaxias CUVIER 1817.

Lixagasa Scotт 1926: s./g. de Saxilaga S. 1926.

Lyragalaxias WHITLE 1935.

Mesites JenYNS 1842: pré-employé, syn. de Galaxias C. 1817.

Neochanna GÜNTHER 1867.

Nesogalaxias WHItLEY 1935.

Paragalaxias ScotT 1935.

Querigalaxias WHITLEY 1935.

Saxilaga Scotт 1926.

\section{FAM. DES APLOCHITONIDAE}

Aplochiton JeNYNS 1842.

Farionella CUVIER et VALENCIENNES 1849 : syn. d'Aplochiton J. 1842.

Haplochiton Agassiz 1846: emend. pro Aplochiton J. 1842.

Lovettia MC CULIoch 1915.

Prototroctes GÜNTHER 1864.

Sous-ordre des STOMIATOIDEI

FAM. DES GONOSTOMATIDAE

$$
\text { (= Gonostomidae) }
$$

Bonapartia Goode et BEAN 1895: pré-employé, syn. de Zaphotias G. et B. 1898 .

Cyclothone Goode et BEAN 1882.

Danaphos BRUUN 1931.

Gonostoma RAFINESQUE 1810.

Manducus GoODE et BEAN 1895 : pré-employé, syn. de Paraphotichthys W. 1931.

Narooma WhItLEY 1935. 
Neophos MYERS 1932.

Neostoma Vaillant : non valide.

Neostoma Filhol. 1884.

Paraphotichthys WHITLEY 1931.

Phosichthys HutTon et Hector 1872.

Photichthys HutTon 1873: emend. pro Phosichthys H. et H. 1872.

Pollichthys GREY 1959.

Polymetone Mc Culi OcH 1926.

Sigmops GILL 1883.

Sonoda GReY 1959.

Thorophos BRUUN 1931.

Triplophos BRAUER 1902.

Woodsia GREY 1959.

Yarella GoODE et BEAN 1895.

Zaphotias Goode et BEAN 1898.

FAMILLE DES IDIACANTHIDAE

Bathyophis GüNTHER 1878.

Idiacanthus PETERS 1876.

Stylophthalmella Roule et ANGEL 1930.

Stylophthalmus BRAUER 1906: serait une forme larvaire d'Idiacanthus P. 1876.

FAMILLE DES MAUROLICIDAE Argyripnus Gilbert et Cramer . 1897.

Coccia GüNTHer 1864: syn. d'Ichthyococcus B. 1840 .

Diplophos GüNTHER 1873.

Ichthyococcus BONAPARTE 1840.

Lychnopoles GARMAN 1899.

Maurolicus Cocco 1838.

Triareus WAITE 1910.

Valenciennellus JORDAN et EVERMANN 1895.

Vinciguerria JoRDAN et EVERMANN 1895.

Zalarges JoRDAN et WILliams 1895: syn. de Vinciguerria J. et E. 1895.
FAM. DES STERNOPTYCHIDAE (Haches d'argent)

Acanthopolyipnus FOWLER 1934: s./g. de Polyipnus G. 1887.

Argyropelecus Cocco 1829.

Margrethia JESPERSEN et TäNING 1919.

Pleurothyris Lowe 1843 : syn. d'Argyropelecus C. 1828.

Pleurothysis GüNTHER: errat. pro Pleurothyris L. 1843.

Polyipnus GüNTHER 1887.

Snellius Koumans 1953.

Sternoptix Hermann 1781.

Sternoptychides OGILBY 1889.

Sternoptyx: vide Sternoptix H. 1781.

FAM. DES ASTRONESTHIDAE

Astronesthes RICHARDSON 1844.

Bathylychnus BRAUER 1902.

Borostomias REgAN 1908.

Diplolychnus Regan et Trewavas 1929.

Heterophotus Regan et Trewavas 1929.

Neonesthes REgan et Trewavas 1929.

Phaenodon Lowe 1850: syn. d'Astronesthes B. 1844.

Rhadinestes REgAN et Trewavas 1929.

Stomianodon BLEEKER 1849 : syn. d'Astronesthes R. 1844.

Warreenula WHITLEY 1941: s./g. d'Astronesthes R. 1844.

F.AMILLE DES

STYLOPHTHALMOIDAE

Stylophthalmoides SANZO 1922.

Stylophthalmus BRAUER 1906: vide Idiacanthidae.

FAMILLE DES CHAULIODIDAE (= Chauliodontidae) 
Chauliodus BLOCH et SCHNEIDER 1801.

Leptodes Swainson 1839: syn. de Chauliodus B. et S. 1801.

Vipera CATESBY 1771 : non valide.

\section{FAMILLE DES STOMIATIDAE}

Achirostomias Regan et Trewavas 1930 : s./g. d'Eustomias FILHOL 1884.

Aristostomias ZUGMAYER 1913.

Bathophilus GigLIOLI 1883.

Bathyembryx BEEBE 1934.

Bathysphaera BEEBE 1932.

Benthalbella ZugmaYer 1911.

Chirostomias Regan et Trewavas 1930.

Dactylostomias GARMAN 1899.

Dinematochirus REgAN et TREWAVAS 1930: s./g. d'Eustomias F. 1884 .

Dolichostomias PARR 1927 : s./g. de Photonectes G. 1887.

Echiostoma LOWE 1843.

Elapterostomias FOWLER 1934.

Eustomias FILHOL 1884.

Eustomias Vaillant 1888.

Flagellostomias PARR 1927.

Gnathostomias PAPPENHEIM 1914.

Grammatostomias GoODE et BEAN 1895.

Grammatostomias HoLT et BYRNE 1913 : pré-employé, syn. de Lamprotoxus H. et B. 1913.

Haploclonus Regan et Trewavas 1930: s./g. d'Eustomias F. 1884.

Haplostomias Regan et Trewavas 1930.

Hyperchoristius GILL 1883.

Lamprotoxus HOLT et BYRNE 1913.

Leptostomias GILBERT 1905.

Lucifer DöDERLEIN 1882 : pré-employé, syn. de Photonectes G. 1887.
Macrostomias BRAUER 1902.

Macrostomoides FOWLER 1958.

Melanonectes Regan et Trewavas 1930: s./g. de Photonectes G. 1887.

Melanostomias BRAUER 1902.

Microchirichthys REGAN et TREWAVAS $1930:$ s./g. de Photonectes G. 1887.

Microdontostomias FOWLER 1934.

Nasistomias Koefoed 1956.

Nematostomias ZugMaYer 1911.

Neostomias GILCHRIST 1906.

Nominostomias Regan et TrewaVAS $1930:$ s./g. d'Eustomias P. 1884 .

Notopodichthys Regan et TrewaVAS 1930: s./g. de Bathophilus G. 1883 .

Odontostomias Norman 1930.

Opostomias GÜNTHER 1887.

Pachystomias GüNTHER 1887.

Parastomias Roule et ANGel 1931.

Pareustomias BAILLY 1931.

Photonectes GÜNTHER 1887.

Photonectoides Koefoed 1956.

Photonectops Chapman 1939.

Photostomias Collett 1889.

Pseudeustomias FOWLER 1934.

Rhynchostomias REGAN et TREWAVAS 1930 : s./g. d'Eustomias' F. 1884.

Spilostomias Regan et Trewavas 1930: s./g. d'Eustomias F. 1884.

Stomias CUVIER 1817.

Stomiasunculus Kaup 1860: larve de Stomias C. 1817.

Stomioides PARR 1933.

Tactostoma Bolin 1939.

Thaumastomias AlCOCK 1890.

Thysanichthys REgAN et TREWAVAS 1930.

Trachinostomias PARR 1927: s./g. de Photonectes G. 1887. 
Trichochirus Regan et Trewavas 1930: s./g. de Bathophilus G. 1883.

Trichostomias ZUGMAYER 1911.

Triclonostomias REGAN et TREWAVAS $1930:$ s./g. d'Eustomias F. 1884.

Trigonolampa Regan et Trewavas 1930.

Urostomias REgAN et TrewaVAS 1930: s./g. d'Eustomias F. 1884.

Zastomias GILBERT 1915.

FAMILLE DES ANOTOPTERIDAE Anotopterus ZugmaYer 1911.

FAMILLE DES ROSAURIDAE

Rosaura TUCKER 1954.

\section{Sous-ordre des OSTEOGLOSSOIDEI}

FAM. DES OSTEOGLOSSIDAE

Delsmania Fowler 1934: s./g. de Scleropages G. 1864.

Ischnosoma SPIX 1829: syn. d'Osteoglossum (V.) C. 1829.

Osteoglossum (VANDELli) CUVIER 1829.

Scleropages GÜNTHER 1864.

\section{FAMILLE DES ARAPAIMIDAE} (VASTRÈs)

Arapaima Müller 1843.

Sudis Cuvier 1817 : pré-employé, syn. d'Arapaima M. 1843.

Vastres CuVIER et VALENCIENNES 1846 : syn. d'Arapaima M. 1843.

\section{FAMILLE DES CLUPISUDIDAE} (= Heterotidae $)$

Clupisudis SwaINSON 1839.

Helicobranchus HYRTL 1854: syn. de Clupisudis S. 1838.
Heterotis (EHRENBERg) MüLLER 1854: syn. de Clupisudis S. 1839.

FAM. DES PANTODONTIDAE (Poisson-papillon)

Pantodon Peters 1877.

Sous-ordre des MORMYROIDEI

FAMILLE DES MORMYRIDAE

Campylomormyrus BLEEKER 1874.

Genyomyrus BOULENGER 1898.

Gnathonemus GILL 1862.

Heteromormyrus STEINDACHNER 1866.

Hippopotamyrus PAPPENHEIM 1906. Hyperopisus GILL 1862.

Isichthys GILL 1862.

Marcusenius GILL 1862.

Mormyrodes GILL 1862.

Mormyrops Müller 1843.

Mormyrus LINNÉ 1758.

Myomyrus BOULENGER 1898.

Oxymormyrus BLEEKER 1874.

Paramyomyrus Pellegrin 1927.

Petrocephalus MARCUSEN 1854.

Phagrus MARCUSEN 1854.

Scrophicephalus SwaINSON 1839 : syn. de Mormyrus L. 1758.

Solenomormyrus BLEEKER 1874.

Stomatorhinus BOULENGER 1898.

FAMILLE DES GYMNARCHIDAE (SUYO)

Gymnarchus CUVIER 1829.

\section{Sous-ordre des GONORHYNCHOIDEI}

FAM. DES GONORHYNCHIDAE Gonorhynchus GroNOw 1763: non valide. 
Gonorhynchus (G.) SCOPOLI 1777. Rhynchana RICHARDSON 1844: syn. de Gonorhynchus (G.) S. 1777.

\section{Sous-ordre des ESOXOIDEI}

\section{FAMILLE DES ESOCIDAE (Brochets ; Pikes)}

Esox LINNÉ 1758.

Kenoza Jordan et EVERMAnn 1896. Lucius GeOFFroY-ST-HILAIRE 1767 : syn. d'Esox L. 1758.

Lucius KLEIN 1775 : non valide.

Lucius RAFINESQUE 1810: syn. Mascalongus JORDAN 1878.

Picorellus RAFINESQUE 1820 : non valide.

\section{FAMILLE DES UMBRIDAE}

(Poisson-chien ou Ombre ; Mud-minnows)

Lacustris Gross 1794 : syn. de $\boldsymbol{U}$ mbra (G.) S. 1777.

Melanura AgAssIz 1853 : syn. de $\boldsymbol{U} \boldsymbol{m}$ bra (G.) S. 1777.

Novumbra Schultz 1929.

Umbra (KRAMER) GRONOW 1763 : non valide.

Umbra (GRonow) SCOPOLI 1777.

FAMILLE DES DALLIIDAE (Alaska Black-fish)

Dallia BEAN 1879.

\section{Sous-ordre des MYCTOPHOIDEI}

\section{FAMILLE DES AULOPIDAE}

Aulopus CUVIER 1817.

Hime Starks 1924.

Latropiscis WHITLEY 1931. Scopelosaurus BLEEKER 1860.
FAMILLE DES SYNODIDAE

(Synodontidae) (Liza:d-fishes)

Allouarnia Whitley 1937: syn. de Xystodus O. 1910.

Alpismaris Risso 1826: larve de Synodus (G.) S. 1777.

Austrotirus Whitley 1937: s./g. de Synodus (G.) S. 1777.

Bathylaco GOODE et BEAN 1895: (Bathylaconidae PARR 1948).

Bathysaurops FOWLER 1938.

Bathysaurus GÜNTHER 1878.

Esosynodus WhItLEY 1937 : s./g. de Synodus (G.) S. 1777.

Exotirichthys WHITLEY 1937: s./g. de Synodus (G.) S. 1777.

Goodella OGILby 1897 : syn. de Trachinocephalus G. 1861.

Laurida Swainson 1839: syn. de Synodus (G.) S. 1777.

Macromystax : juvénile de Bathylaco G. et B. 1895 .

Negotirus WhItLey 1937: s./g. de Synodus (G.) S. 1777.

Newtonscottia WhItLEY 1937: s./g. de Synodus (G.) S. 1777.

Pelopsia Facciola 1883 : vide Chlorophthalmidae.

Saurida CUVIER et VALENCIENNES 1849.

Saurus CATESBY 1771: non valide.

Saurus CUVIER 1817.

Soarus LINCK 1790: non valide.

Synodus Gronow 1763 : non valide.

Synodus (Gronow) Scopoli 1777.

Tirus Rafinesque 1810: syn. de $\boldsymbol{S} \boldsymbol{y}$ nodus (G.) S. 1777.

Trachinocephalus GILL 1861.

Xystodus OgILby 1910.

\section{FAM. DES HARPODONTIDAE (Bombay-ducks)}

Harpodon LesUeUR 1825.

Peltharpodon FOWLER 1934.

Sauridichthys BLEEKER 1858: syn. de Harpodon S. 1825.

Triurus SwaInson 1839: syn. d'Harpodon S. 1825. 
FAMILLE DES

CHLOROPHTHALMIDAE

Bathysaurops FOWLER 1938.

Bathysauropsis REGAN 1911.

Chlorophthalmus BONAPARTE 1840.

Hyphalonedrus GoOdE 1880.

Pelopsia FAcCIOLA 1883: forme larvaire de Chlorophthalmus B. 1840 .

FAMILLE DES SUDIDAE

$(=$ Paralepidae $=$ Paralepididae $)$

Arctozenus GILL 1864.

Bathymicrops KOEFOED 1910.

Bathysudis PARR 1928: s./g. de Lestidium G. 1905.

Bathytyphlops NYBELIN 1957.

Lestidiops HuвBs 1916.

Lestidium GILBERT 1905.

Lestrolepis HARRY 1953: s./g. de Lestidium G. 1905.

Luciosudis FRASER-BRUNNER 1931.

Macroparalepis EGE 1933.

Magnisudis HARRY 1953.

Notolepis Dollo 1908.

Paralepis CUVIER 1817.

Parasudis REgAN 1911.

Profundisudis HARRY 1953: s./g. de Notolepis D. 1908.

Prymnothonoides WhItLeY et PHILLIPS 1939.

Prymnothonus RICHARDSON 1844 : forme larvaire d'un Sudidae sp.

Skagerakia NYBELIN 1947.

Stemonosudis HARRY 1951.

Sudis RAFInESQUE 1810.

Symproptopterus Cocco 1885.

Uncisudis MAUL 1956.

FAMILLE DES NOTOSUDIDAE

Notosudis WAITE 1916.

\section{FAMILLE DES MYCTOPHIIDAE (= Scopelidae)}

(Poissons-lampes ; Lantern-fishes)
Aethoprora Goode et BEAN 1895 . Alysia Löwe 1839: syn. de Rhinoscopelus L. 1892.

Benthosema GoOdE et BEAN 1895.

Catablemella EIGENMANN et EIGENManN 1890: syn. de Notoscopelus G. 1864.

Cavelampus WhITLEY 1933: s./g. de Collettia G. et B. 1895.

Centrobranchus FOWLER 1904.

Collettia Goode et BEAN 1895.

Ctenoscopelus FRASER-BRUNNER 1947.

Cyphoscopelus FOWLER 1925.

Cyrtorhynchus CosTa 1855.

Dasyscopelus GüNTHER 1864.

Diaphus Eigenmann et EigenmanN 1891.

Diogenichthys BOLIN 1939.

Elampa FraSER-BRUNNER 1949 : syn. d'Esox L. 1758.

d'Elampadena W. 1953.

Elampadena WHITLEY 1953: s./g. d'Electrona G. et B. 1895.

Electrona GOODE et BEAN 1895.

Goniichthys WhITLEY 1933: syn. de Rhinoscopelus L. 1892.

Gymnoscopelus GüNTHER 1873.

Hietops Fraser-BrunNer 1949.

Hintonia Fraser-BRUNNER 1949.

Hyperphotops FRASER-BRUNNER

1949: s./g. de Diaphus E. et E. 1891 .

Lampadena GoODE et BEAN 1895.

Lampanyctodes FRASER-BRUNNER 1949.

Lampanyctus BONAPARTE 1840.

Lampichthys FRASER-BRUNNER 1949.

Lamprossa JORDAN et HUBBS 1925.

Lepidophanes FRASER-BRUNNER

1949: s./g. de Lampanyctus B. 1840 .

Lowenia FoWLER 1925: s./g. de Myctophum R. 1810.

\section{Lobianchia}

Lychnophora FRASER-BRUNNER 1949: s./g. de Lampadena G. et B. 1895 . 
Macrostoma Risso 1826 : pré-employé, syn. de Notoscopelus G. 1864 .

Myctophum RAFINESQUE 1810.

Mystibranchus WHITLEY 1939.

Nannobrachium GüNTHER 1887 : syn. de Lampanyetus B. 1840.

Nasolychnus SмIтH 1933: s./g. de Myctophum R. 1810.

Neoscopelus JoHnson 1853.

Neoscopelus Castelnau 1875.

Notolychnus Fraser-BRUNNER 1949.

Notoscopelus GüNTHER 1864.

Nyctimaster JORDAN 1922.

Nyctophus Cocco 1829: syn. de Myctophum R. 1810.

Pantophos Jordan et HubBs 1925.

Phanerobranchus Cocco 1846.

Proctomyctophum FRASER-BRUNNER 1949: s./g. d'Electrona G. et B. 1895 .

Protoblepharon STECHe 1909.

Rhinoscopelus LÜTKEN 1892.

Scopelengys Alcock 1890.

Scopelogenys: vide Scopelengys A. 1890.

Scopelopsis BRAUER 1906.

Scopelus Cuvier 1817: syn. de Myctophum R. 1810.

Serpa Whitley 1933 : syn. de Lampanyctus B. 1840.

Solivomer MILler 1947.

Stenobrachius EIGENMANN et EIGENMANN 1890 : syn. de Nannobrachium G. 1840 .

Symbolophorus BOLIN et WISNER 1959.

Taaningichthys BoLIN 1959.

Tarletonbeania EIGENMANN et EIGENMANN 1891.

Triphoturus FRASER-BRUNNER 1949: s./g. de Lampanyctus B. 1840.

Vestula BoLIN 1946.
Sous-ordre des ALEPISAUROIDEI

FAM. DES SCOPELARCHIDAE (= Evermanellidae $=$ Odontostomidae)

Coccorella Roule 1929.

Dissomma BRAUER 1902.

Evermanella FOWLER 1901.

Neoscopelarchoides CHAPMAN 1939.

Odontostomops FOWLER 1934: s./g. d'Evermanella F. 1901.

Odontostomus BORODINE : pré-employé, syn. d'Evermanella F. 1901.

Promacheon WeBer 1913.

Scopelarchoides PARR 1929.

Scopelarchus ALcocK 1896.

\section{FAMILLE DES IPNOPIDAE}

Ipnoceps FOWLER 1943.

Ipnops GÜNTHER 1878.

FAMILLE DES ALEPISAURIDAE (= Plagiodontidae) (Hand-saw fish) Alepidosaurus: syn. d'Alepisaurus L. 1833.

Alepisaurus Lowe 1833.

Caulopus GILL 1862.

Eugnathosaurus REGAN 1913.

Plagyodus (Steller) Pallas 1811: non valide.

Plagyodus (S.) GüNTHer 1867: syn. d'Alepisaurus L. 1833.

FAMILLE DES MALACOSTEIDAE Malacosteus AYRES 1849.

Ultimostomias BEEBE 1933.

FAM. DES BATHYPTEROIDAE

Bathypterois GÜNTHER 1878.

Belonopterois ROULE 1916.

Hemipterois REgAN 1911.

Synapteretmus GoOdE et BEAN 1895. 
FAM. DES BENTHOSAURIDAE Benthosaurus GoODE et BEAN 1886.

FAMILLE DES OMOSUDIDAE Omosudis GÜNTHER 1887.

Sous-ordre des ATELEOPOIDEI

FAMILLE DES ATELEOPIDAE

Ateleopus Temminck et Schlegel 1846: si pré-employé, serait remplacé par Podateles B. 1902.

Ijimaia SAUTER 1905.

Melanogloea BARNARD 1941.

Parateleopus SMITH et RADCLIFFe 1912.

Podateles BOULENGER 1902: syn. d'Ateleopus T. et S. 1846.

Sous-ordre des MIRAPINNATOIDEI

FAMILLE DES MIRAPINNIDAE

Mirapinna Bertelsen et MARShall 1956.

FAM. DES EUTAENIOPHORIDAE Eutaeniophorus BERTELSEN et MARSHALL 1958.

Parataeniophorus BERTELSEN et MaRshall 1956.

Taeniophorus BERTELSEN et MARSHALL 1956: pré-employé, syn. de Eutaeniophorus B. et M. 1958.

FAM. DES BARBOURISIIDAE

Insertae sedis (classée soit près des Myctophiidae, soit près des Cetomimidae)

Barbourisia PARR 1945.

Bathysidus BEEBE 1934 : Clupéiforme Insertae sedis.

\section{Ordre des TETRAODONTIFORMES}

\section{Sous-ordre des \\ OSTRACIONOIDEI}

FAMILLE DES OSTRACIONIDAE (Coffres)

Acanthostracion BLEEKER 1866.

Anoplocapros KaUP 1855.

Aracanostracion SМITH 1949.

Caprichthys Mc Culloch et WaIte 1915.

Capropygia GRAY 1855.

Chapinus Jordan et EvermanN 1896.

Cibotion (Klein) Kaup 1855: syn. d'Ostracion L. 1758.

Goniodermus RAFINESQUE 1815 : syn. d'Ostracion L. 1758.

Kentrocapros KaUP 1855.

Lactophrys SwaINSON 1839.

Lactoria JoRDAN et FowLER 1902.

Ostracion LINNÉ 1758.

Paracanthostracion WHITLEY 1933. Rhinesomus Swainson 1839: syn. de Lactophrys S. 1839.

Rhynchostracion FRASER-BRUNNER 1935.

Strophiurichthys FRASER-BRUNNER 1935.

Tetrosomus SWaInson 1839: s./g. d'Ostracion L. 1758.

Triorus Jordan et HubBs 1925.

FAMILLE DES ARACANIDAE

Acarana Gray 1833 : errat. pro Aracana G. 1838 .

Aracana Gray 1838:

Platycanthus SwaInson 1839: syn. d'Aracana G. 1833.

FAM. DES CANTHIGASTERIDAE Anosmius Peters 1855: syn. de Canthigaster S. 1839.

ANN. PARASITO. HUM. ET COMP., 1962, 37, $\mathrm{n}^{\circ} 6^{\mathrm{bis}}$ (fasc. suppl.) 
Caenthigaster JORDAN 1919 : errat. pro Canthigaster S. 1839.

Canthigaster SwaInson 1839.

Eumycterias JENKINS 1901: pré-employé, syn. de Lucubrapiscis W. 1931.

Lucubrapiscis WHITLEY 1931: syn. ou s./g. de Canthigaster S. 1839.

Prilonotus (KAUP) MILler 1854: syn. de Canthigaster S. 1839.

Psilonotus Swainson 1839: syn. de Canthigaster S. 1839.

Rhynchotus BIBRON 1855: syn. de Canthigaster S. 1839.

Tropidichthys BLEEKER 1854: syn. de Canthigaster S. 1839.

\section{Sous-ordre des TRIODONTOIDEI}

FAMILLE DES TRIODONTIDAE Triodon CUVIER 1829.

\section{Sous-ordre des TETRAODONTOIDEI}

FAM. DES TETRAODONTIDAE (Poissons-globes)

Akamefugu ABE 1954: s./g. de Fugu A. 1952.

Anchisomus KaUP 1854: syn. de Sphoeroides L. 1798.

Aphanacanthus BIBRON 1855: syn. de Leiodon S. 1839.

Apsicephalus Hollard 1857 : syn. de Dichotomycter B. 1855.

Arothron MüLLER 1839 : s./g. d'Ovoides L. 1758.

Boesemanichthys ABE 1952.

Brachycephalus HollaRd 1857 : syn. de Tetraodon L. 1758.

Carinotetraodon BENL 1957.

Cheilichthys Müller 1839: syn. de Sphoeroides L. 1798.

Chelonodon MüLLER 1839.
Chelonodontops SMITH 1958.

Cirrhisomus SwaInson 1839: syn. de Sphoeroides L. 1798.

Contusus WhITLEY 1947.

Crayracion (KLEIN) BLEEKER 1865 : syn. de Tetraodon L. 1758 et d'Ovoides L. 1798.

Cyprichthys WHITLEY 1936.

Dichotomycter HollaRD 1857: syn. de Dichrotomycter B. 1855.

Dichotomycterus Troschel 1856 : syn. de Dichrotomycter B. 1855 .

Dichrotomycter BIBRON 1855.

Dilobomycter BIBRON 1855: syn. d'Ovoides L. 1798.

Ephippion BIBRON 1855: pré-employé, syn. d'Hemiconiatus G. 1870.

Epipedorhynchus BIBRON 1855: syn. d'Ovoides L. 1798.

Fugu ABE 1952.

Guentheridia GILBERT et STARKS 1904.

Hemiconiatus GüNTHER 1870.

Higanfugu ABE 1954 : s./g. de Fugu A. 1952.

Holacanthus Gronow 1854 : pré-employé, syn. de Tetraodon L. 1758.

Kankuda HORA 1924.

Leiodon SWAINSON 1839.

Leiodon OWEN 1841: syn. de Leiodon S. 1839.

Leisomus SwaInson 1839: pré-employé, syn. de Leiodon S. 1839.

Lepidorbidus FOWLER 1929: s./g. de Sphoeroides L. 1798.

Liosaccus GÜNTHER 1870.

Monotreta BIBRoN 1855: syn. de Leiodon S. 1839.

Monotretus TrosCHEL 1856: emend. pro Monotreta B. 1855.

Omegophora WHITLEY 1934.

Oonidus RAFINESQUE 1815: syn. d'Ovoides L. 1798.

Orbidus RAFINESQUe 1815: syn. de Sphoeroides L. 1798.

Orbis CATESBY 1771 : non valide.

Orbis (Plumier) DE LACÉPÈde 1800 : non valide, syn. de Tetraodon L. 1758. 
Orbis FISCHER 1813 : non valide, syn. d'Ovoides L. 1798.

Ovoides DE LACÉPÈDE 1798.

Ovoides Cuvier 1800: syn. d'Ovoides L. 1798.

Ovoides DumÉRIL 1806 : syn. d'Ovoides L. 1798.

Ovum BLOCH et SCHNEIDER 1801.

Proodus Fowler 1944.

Shippofugu ABE 1949: s./g. de Sphoeroides L. 1798.

Shosaifugu ABE 1949: s./g. de Sphoeroides L. 1798.

Spheroides DumÉRIL 1806: syn. de Sphoeroides L. 1798.

Sphoeroides DE LACÉPÈDE 1798.

Stenometopus BIBRON 1855: syn. de Sphoeroides L. 1798.

Takifugu Aве 1949: s./g. de Sphoeroides L. 1798.

Tetraodon LINNÉ 1758.

Tetrodon LINNÉ 1766: vide Tetraodon L. 1758.

Thecapteryx FOWLER 1948.

Torafugu nom. nud.: syn. de Fugu A. 1954.

Torquigener WHITLEY 1930.

Torquiniger : errat. pro Torquigener W. 1930.

Uranostoma (BIBRON) BLEEKER 1865: non valide, syn. de Tetraodon L. 1758 ou de Sphoeroides L. 1798 .

\section{FAM. DES CHONERHINIDAE}

$$
\text { (= Xenopteridae) }
$$

Choenorhinos: vide Chonerhinos B. 1854.

Chonerhinos BLEEKER 1854.

Chonerhinus: vide Chonerhinos B. 1834.

Xenopterus BIBRoN 1855 : syn. de Chonerhinos B. 1854.

\section{FAM. DES LAGOCEPHALIDAE}

Amblyrhynchotus BIBRON 1855.

Catophorhynchus BIBRoN 1855: syn. de Lagocephalus S. 1839.
Gastrophysus MüLleR 1843 : préemployé, syn. de Lagocephalus S. 1839.

Geneion BIBron 1855: syn. de Lagocephalus S. 1839.

Lagocephalus SWAINSON 1839.

Lagrocephalus JoRDAN 1919: errat. pro Lagocephalus S. 1839.

Physogaster MülLeR 1839: syn. de Lagocephalus S. 1839.

Pleuranacanthus (BIBRON) BLEEKER 1865 : non valide, syn. de Lagocephalus S. 1839.

Promecocephalus BIBRON 1855: syn. de Lagocephalus S. 1839.

\section{FAMILLE DES DIODONTIDAE (Porcs-épics)}

Allomycterus Mc Culloch 1921.

Atopomycterus (Verreaux) BleEKER 1865.

Cephalopsis RAFINESQUE 1815: syn. de Diodon L. 1758.

Chilomycterus BIBRON 1846.

Cyanichthys KaUP 1855: syn. de Chilomycterus B. 1846.

Cyclichthys KaUP 1855.

Dicotylichthys KAUP 1855.

Diodon LINNÉ 1758.

Euchilomycterus WAITE 1900.

Lophiodon FRASER-BRUNNER 1943 : s./g. de Diodon L. 1758.

Lyosphaera EVERMANN et KENDALL 1898.

Orbis Mülleer 1767: non valide.

Paradiodon BleEKer 1865: syn. de Diodon L. 1758.

Tragulichthys WHITLEY 1931.

Trichocyclus GüNTHER 1870: larve de Diodon L. 1758.

Trichodiodon BLEEKER 1866: larve de Diodon L. 1758.

FAMILLE DES COLOMESIDAE

Batrachops BIBRoN 1855: syn. de Colomesus G. 1885.

Colomesus GILL 1885. 
Sous-ordre des MOLOIDEI

FAMILLE DES MOLIDAE (Orthagoriscidae)

(Poissons-lunes ; Sun-fishes) Acanthosoma DeKay 1842: syn. de Mola K. 1770.

Aledon De Castelnau 1861 : syn. de Mola K. 1770.

Centaurus Kaup 1855 : larve de Mola K. 1770.

Cephalus SHaw 1804: syn. de Mola K. 1770.

Chelonopsis JoRdAN 1900: non valide. Diplanchias RAFINESQUE 1810: syn. de Mola K. 1770.

Masturus GILL 1884.

Mola KölReuther 1770.

Mola LincK 1790: syn. de Mola K. 1770.

Mola Cuvier 1798 : syn. de Mola K. 1770.

Molacanthus SwaINSON 1839: syn. de Mola K. 1770.

Orthagoriscus BLOCH et SCHNEIDER 1801: syn. de Mola K. 1770.

Orthagus RAFINESQUe 1810: syn. de Mola K. 1770.

Ozodura RAnzani 1839 : syn. de Mola K. 1770.

Pallasia NARdo 1839: larve de Mola K. 1770.

Pedalion Swainson 1838: syn. de Mola K. 1770.

Pseudomola CADENAT 1959.

Ranzania NARDO 1839.

Trematopsis RANZANI 1839: syn. de Mola K. 1770.

Triurus DE LACÉPÈdE 1800 : syn. de Mola K. 1770.

Tympanomium RANZANI 1839: syn. de Mola K. 1770.

\section{Ordre des \\ SACCOPHARYNGIFORMES}

FAMILLE DES

$S A C C O P H A R Y N G I D A E$ (Gulpers)
Ophiognathus HaRdwood 1827 : syn. de Saccopharynx M. 1824.

Saccopharynx MrtchILl 1824.

FAM. DES EURYPHARYNGIDAE Eurypharynx VAILlANT 1882.

Gastrostomus GILL et RYDER 1883. Jordanites FOWLER 1925: s./g. d'Eurypharynx V. 1882.

Macropharynx BRAUER 1902.

Megalopharynx BRAUER 1900: préemployé, syn. de Macropharynx B. 1902.

Rouleina FowleR 1925: pré-employé, syn. de Jordanites F. 1925.

\section{Ordre des \\ MONOGNATHIFORMES}

FAM. DES MONOGNATHIDAE

Monognathus BERTIN 1926.

Phasmatostoma MYERS 1940.

\section{Ordre des CYPRINIFORMES}

\section{Sous-ordre des CHARACOIDEI}

FAMILLE DES CHARACIDAE (= Characinidae) (Characins)

Acanthocharax EIGENMANN 1912 Acestrocephalus Eigenmann 1903. Acestrorhamphus EIGENMANN 1903. Acestrorhynchus EIGENMANN 1903. Acnodon Eigenmann 1903.

Acrobrycon PeARSON 1924.

Aequidens Steindachner 1915 : préemployé, syn. d'Evemichthys W. 1935.

Agoniates Müller et Troschel 1845. 
Alestes Müller et Troschel 1844. Alestiops Hoedeman 1951: s./g. d'Alestes M. et T. 1844.

Alestobrycon Hoedeman 1951.

Alestogrammus HoEdeman 1951: s./g. d'Alestopetersius H. 1951. Alestopetersius Hoedeman 1951. Amblystilbe FowLER 1940.

Anacyrtus GüNTHER 1864: syn. de Characinus L. 1758.

Anoptichthys HubBs et INNEs 1936 : syn. d'Astyanax B. et G. 1854. Aphyocharax GüNTHER 1868. Aphyocheirodon EIGENMANN 1915. Aphyodite Eigenmann 1912. Apistogramma REGAN 1913. Apodastyanax FoWLER 1911. Argonectes BöHLKE et MYers 1956. Argopleura Eigenmann 1913. Arnoldichthys MYERS 1926. Asiphonichthys COPE 1894. Astyacinus EIGENMANN 1907. Astyanax BAIRD et GIRARD 1854. Atopomesus MYERS 1927. Aulixidens BöHLKE 1952.

Autanichthys FERNANDEZ-YEPEZ 1950.

Bario MYERS 1940.

Bathyaethiops FOWLER 1949. Bergia STEINDACHNeR 1891: pré-employé, syn. de Pseudocorynopoma P. 1891.

Bertoniolus FOWLER 1918.

Bivibranchia EIGENMANN 1912. Bleptonema EIGENMANN 1914: syn. de Prionobrama F. 1913.

Boulengerella EIGENMANN 1903.

Brachyalestes GÜNTHER 1864.

Brachychalcinus BOULENGER 1892.

Brachypetersius HOEDEMAN 1955: s./g. d'Alestopetersius H. 1951.

Bramocharax GILL 1877.

Brycinus Cuvier et VALENCIENNES 1849.

Brycochandus EIGENMANN 1908.

Brycon Müller et Troschel 1844.
Bryconacidnus EIGENMANN et MYERS 1929.

Bryconaethiops GÜNTHER 1873.

Bryconalestes HoEDEMAN 1951.

Bryconamericus EIGENMANN 1907.

Bryconodon EIGENMANN 1903.

Bryconops KNER 1859.

Camposichthys Travassos 1946.

Carlana Strand 1928.

Carlia Meek 1914 : pré-employé, syn. de Carlana S. 1928.

Catabasis EIGENMANN et NORRIS 1900.

Catoprion MüLler et Troschel 1844.

Ceratobranchia EIGENMANN 1914.

Chalceus CUVIER 1817.

Chalcinopelecus HOLMBERG 1891 : syn. de Pseudocorynopoma P. 1891.

Chalcinopsis KNER et STEINDACHNER 1863 : syn. de Brycon M. et T. 1844.

Chalcinopsis Holmberg 1891: syn. de Pseudocorynopoma P. 1891.

Chalcinus Cuvier et VALENCIENNES 1849 : pré-employé, syn. de Triportheus C. 1872.

Chalcinus RAFINESQUE.

Champsoborus BOULENGER 1909.

Characinus LINNÉ 1758.

Characinus DE LACÉPÈDE 1803 : syn. de Characinus L. 1758.

Characinus Cuvier 1817: syn. de Characinus L. 1758.

Charax Gronow 1763: non valide.

Charaxodon FERNANDEZ-YEPEZ 1947.

Cheirodon GIRARD 1854.

Cheirodontops ScHULTZ 1944.

Chirodon GüNTHER 1864 : vide Cheirodon $G .1854$.

Clupeacharax PEARSON 1924.

Clupeocharax PELlegrin 1926: préemployé, syn. de Clupeopetersius P. 1928.

Clupeopetersius PeLLegRIN 1928.

Cnidon Müller et Troschel 1845 . 
Coelurichthys RIBEIRO 1908.

Colossoma EigenManN 1903.

Compsura Eigenmann 1915.

Copeina Fowler 1906.

Copella MYERS 1956.

Corynopoma GILL 1858 : syn. de

Stevardia G. 1858.

Corynopomops FOWLER 1943.

Coscinoxyron FOWLER 1907.

Creagrudite MYERS 1927.

Creagrutops Schultz 1944.

Creagrutus GÜNTHER 1864.

Creatochanes GüNTHER 1864.

Crenuchus GüNTHER 1863.

Ctenobrycon Eigenmann 1908.

Ctenocharax REGAN 1907.

Curimatoides FOWLER 1940.

Cynocharax FOWLER 1907.

Cynodon SPIX 1829.

Cynodon Campos 1946 : pré-employé, syn. de Camposichthys T. 1946.

Cynopotamus CUVIER et VALENCIENNES 1849.

Cyphocharax FOWLeR 1906.

Cyrtocharax Fowler 1907: syn. de Cynopotamus C. et V. 1849.

Dermatocheir DURBIN 1909.

Deuterodon EIGENMANN 1907.

Diapoma COPE 1894.

Distoechus GoMes 1948.

Ectrepopterus FOWLER 1944: s./g. de Megalamphodus E. 1915.

Elachocharax MYERS 1927.

Entomolepis EIGENMANN 1917: préemployé, syn. de Bario M. 1940.

Ephippicharax FOWLER 1913.

Epicyrtus Müller et TrOSCHEL 1844 : pré-employé, syn. de Characinus L. 1758.

Erythrichthys BONAPARTE 1833 : syn. d'Erythrinus (G.) S. 1777.

Erythrinus GRONOW 1763: non valide.

Erythrinus (G.) Scopoli 1777.

Erythrinus Agassiz 1829 : syn. d'Erythrinus (G.) S. 1777.

Eucynopotamus FowLER 1904.
Evemichthys WHITLEY 1935.

Evermannella EIGENMANN 1903 : préemployé, syn. d'Eucynopotamus F. 1904 .

Evermannolus EIGENMANN 1907 : syn. d'Eucynopotamus F. 1904.

Exodon Müller et Troschel 1844 : pré-employé, syn. d'Hystricodon G. 1864.

Fowlerina EIgENMANN 1907 : pré-employé, syn. d'Ephippicharax F. 1913.

Genycharax EIGENMANN 1912.

Gephyrocharax EIGENMANN 1912.

Gilbertella EIGENMANN 1903: préemployé, syn. de Gilbertolus E. 1907.

Gilbertolus EIGENMANN 1907.

Glandulocauda EIGENMANN 1911: syn. de Mimagoniates R. 1907.

Gnathocharax FOWLER 1913.

Grammabrycon FOWLER 1941.

Grundulus Cuvier et VALENCIENNES 1846: syn. de Ctenocharax R. 1907.

Gymnocharacinus STEINDACHNER 1903.

Gymnocorymbus EIGENMANN 1908. Gymnotichthys FERNANDEZ - YEPEZ 1950.

Hasemania ELLIS 1911.

Hemibrycon GüNTHER 1864.

Hemigrammalestes PEL L E G IN 1926.

Hemigrammocharax PELLEGRIN 1923.

Hemigrammonannocharax HoLLY 1930.

Hemigrammopetersius PELLEGRIN 1926.

Hemigrammus GILL 1858.

Henochilus Garman 1890.

Hepsetus Swarnson 1838: syn. de Sarcodaces G. 1864.

Heterocharax EIGENMANN 1912.

Heterogramma REgAN 1906 : pré-employé, syn. d'Apistogramma R. 1913.

Heterythrinus GüNTHER 1864: syn. d'Erythrinus (G.) S. 1777. 
Holesthes JoRDAN 1920: errat. pro Holoshesthes E. 1903.

Hollandichthys EIGENMANN 1910.

Holobrycon EIGENMANN 1909.

Holoprion EIGENMANN 1903: syn. d'Aphiocharax G. 1868.

Holopristis EIgENMANN 1903.

Holoshesthes EIGENMANN 1903.

Holotaxis COPE 1870.

Holotaxis EIGENMANN 1903 : pré-employé, syn. de Copeina F. 1906.

Hoplerythrinus GILL 1895.

Hoplias GILL 1903.

Hydrocion DuMÉRIL 1856: syn. d'Hydrocyon C. 1817.

Hydrocynus DUMÉRIL 1856.

Hydrocyon CUVIER 1817.

Hydrocyonichthys Travassos 1952. Hydrocyonoides DE CASTELNAU 1861 : syn. de Sarcodaces G. 1864.

Hydrolycus Müller et Troschel 1844.

Hydropardus REINHARDT 1849 : syn. de Raphiodon A. 1829.

Hyphessobrycon EIGENMANN 1908.

Hysteronotus EIGENMANN 1911.

Hystricodon GÜNTHER 1864.

Iguanodectes COPE 1872.

Joinvillea STEINDACHNER 1908: syn. de Deuterodon E. 1907.

Knodus EIGENMANN 1911.

Landonia EIgENMANN et HeNn 1914.

Lebiasina CUVIER et VALENCIENNES 1846.

Leptagoniates BOULENGER 1887.

Leptobrycon EIGENMANN 1915.

Lobodeuterodon FOWLER 1945: s./g. de Deuterodon E. 1907.

Lonchogenys MYERS 1927.

Luetkenia STEINDACHNER 1876: préemployé, syn. de Stichonodon E. 1903.

Macrodon MülLeR 1843: pré-employé, syn. de Hoplias G. 1903.

Macropsobrycon EIGENMANN 1915.

Markiana EIGENMANN 1903.
Megalamphodus EIGENMANN 1915. Megalobrycon GüNTHER 1869: syn. de Brycon M. et T. 1844.

Melloina Amaral-CAmpos 1946.

Metynnis COPE 1878.

Micralestes BOULENGER 1899.

Microbrycon EIGENMANN 1914.

Microcaelurus MIRANDA-RIBEIRO 1940.

Microgenys EIgenMann 1913.

Microlepidalestes Hoedeman 1951: s./g. d'Alestopetersius H. 1951.

Microschemobrycon EI G N M AN N 1915.

Mimagoniates REgAN 1907.

Mixobrycon EIGENMANN 1915.

Moenkhausia EIGENMANN 1903.

Monotocheirodon PEARSON 1924.

Moojenichthys RIBEIRO 1957.

Moralesia FOWLER 1943: pré-employé, syn. de Moralesicus F. 1959.

Moralesicus FOWLER 1959.

Myleocollops EIGENMANN 1903.

Mylesinus CUVIER et VALENCIENNES 1949.

Myletes CUVIER 1815.

Myletes MüLler et Troschel 1844: pré-employé, syn. de Myloplus G. 1895 .

Myleus Müller et Troschel 1844. Myloplus GILL 1895.

Mylossoma EIGENMANN 1903.

Nannopetersius HOEDEMAN 1951: s./g. d'Alestopetersius H. 1951.

Nematobrycon EIGENMANN 1911.

Nematopoma Gill 1858: syn. de Stevardia G. 1858.

Neoborus BOULENGER 1899.

Notropocharax MARINI, Nichols et LAMONTE 1933 : syn. de Aphyocharax G. 1868.

Odontostilbe COPE 1870.

Odontostoechus GoMes 1948.

Odontostomus Cocco 1838.

Oligobrycon EIGENMANN 1915.

Oligosarcus GüNTHER 1864. 
Onouphrios WHITLEY 1953.

Ophiocephalops Fowler 1906: syn. d'Hoplerythrinus G. 1895.

Opisthanodus AHL 1935.

Orthomyleus EIGENMANN 1903.

Othonocheirodus MYERS 1927.

Othonophanes EIGENMANN 1903.

Paragoniates STEINDACHNER 1876.

Paramyloplus NoRMAN 1929.

Parastremma EIGENMANN 1912.

Parecbasis Eigenmann 1914.

Paroligosarcus AMARAL-CAMPos et Trewavas 1949: s./g. d'Oligosarcus G. 1864.

Pedalibrycon FowLer 1944.

Pellegrinia FOWLER 1907.

Petersialestes H O D E M N 1951: s./g. d'Alestopetersius H. 1951.

Petersius HILgendorf 1894.

Phanagoniates EIgENMANN et HeNN 1914: syn. de Phenagoniates E. et W. 1914.

Phenacobrycon EIGENMANN 1920.

Phenacogaster EIgENMANN 1907.

Phenacogrammus EIGENMANN 1907: s./g. de Micralestes B. 1899.

Phenagoniates EIgENMANN et WILSON 1914.

Phoxinopsis Regan 1907.

Phoxonopsis INNES 1938: syn. de Spintherobolus E. 1911.

Piabarchus MYERS 1929.

Piabina ReInHARdT 1866.

Piabuca : vide Piabucus (C.) O. 1817.

Piabucidium MYERS 1929.

Piabucina CuVIER et VAlENCIENNES 1849.

Piabucus (CuVIER) OKEN 1817.

Piaractus EIgenManN 1903.

Planaltina BöHLKE 1954.

Plethodectes COPE 1870: syn. de Chalceus C. 1817.

Poecilocharax EIGENMANN 1909.

Poecilurichthys GILL 1868: s./g. d'Astyanax B. et G. 1854.
Poptella Eigenmann 1908.

Prionobrama FoWLER 1913.

Pristella EIgENMANN 1908.

Pristicharax FOWLER 1949.

Pristobrycon EIGENMANN 1915.

Probolodus EigenManN 1911.

Prodontocharax PEARSON 1924.

Proportheus COPE 1872.

Psalidodon EIGENMANN 1911.

Psalidostoma KNER 1864: pré-employé, syn. de Onouphrios W. 1953.

Psellogrammus EIgENMANN 1908.

Pseuderythrinus HoEdeman 1950.

Pseudochalceus KNER 1863.

Pseudocheirodon MEEK et HILDEBRAND 1916.

Pseudocorynopoma Perugia 1891.

Pterobrycon EIgenManN 1913.

Pterohemiodus Fowler 1940.

Pygocentrus Müller et Troschel 1844.

Pygopristis Müller et Troschel 1844.

Pyrrhulina CUVIER et VALENCIENNES 1846.

Rachoviscus MYERS 1926.

Ramirezella FERNANDEZ-YEPEZ 1949.

Raphiodontichthys AMARAL-CAMPOS 1945.

Reganina Fowler 1907: syn. de Colossoma E. 1903.

Rhabdalestes HoEDEMAN 1951 : s./g. de Micralestes B. 1899.

Rhabdopetersius HoEdEMAN 1951: s./g. d'Alestopetersius H. 1951.

Rhaphiodon Agassiz 1829: syn. de Cynodon SPIX 1829.

Rhinobrycon MYERS 1944.

Rhoadsia FOWLER 1911.

Roeboides GÜNTHER 1864.

Roeboxodon GÉRY 1959.

Roestes GÜNTHER 1864.

Rooseveltiella EIGENMANN 1915. 
Saccoderma SCHULTZ 1944.

Salminus AgASSIZ 1829.

Sarcodaces GÜNTHER 1864.

Scissor GÜNTHER 1864.

Sealeina FOWLER 1907.

Serrasalmo DUMÉRIL 1805: syn. de Serrasalmus L. 1803.

Serrasalmus DE LACÉPÈDE 1803.

Sphyraenocharax FowLER 1907 : syn. d'Acestrorhamphus E. 1903.

Spintherobolus EIGENMANN 1911.

Starksina Fowler 1907: syn. de Mylossoma E. 1903.

Stethaprion COPE 1870.

Stevardia GILL 1858.

Stichonodon EIGENMANN 1903.

Tetragonopterus CUVIER 1815.

Thayeria EIGENMANN 1907.

Thayeria EIgENMANN 1908.

Thrissobrycon BöHLKE 1953.

Tometes CuVIER et VALENCIENNES 1849: syn. de Myleus M. et T. 1844.

Triportheus COPE 1872.

Triurobrycon EIGENMANN 1909.

Tylobranchus EIGENMANN 1912.

Tyttocharax FOWLER 1913.

Utiaritichthys MIRANDA-RIBEIRO 1937.

Vesicatrus EIgENMANN 1911.

Waiteina Fowler 1907 : syn. de Colossoma E. 1903.

Xenagoniates MYERS 1942.

Xenurobrycon MYers et MIRANDARIBEIRO 1945.

Xenurocharax REGAN 1913.

Xiphocharax FOWLER 1914.

Xiphorhamphus MüLler et TrosCHEL 1844: pré-employé, syn. d'Acestrorhynchus E. 1903.

Xiphorhynchus Agassiz 1829: préemployé, syn. d'Acestrorhynchus E. 1903.

Zygogaster EIGENMANN 1913.
FAM. DES GASTEROPELECIDAE

Carnegiella EIGENMANN 1909.

Gasteropelecus GRoNow 1763: non valide.

Gasteropelecus Pallas 1769.

Pterodiscus EIgenManN 1909: syn. de Gasteropelecus P. 1769.

Thoracocharax FOWLER 1907.

FAMILLE DES XIPHOSTOMIDAE Apolinarella FernaNDEZ-YEPEZ 1948.

Belonocharax Fowlen 1907 : syn. de Ctenolucius G. 1861.

Bitricarinata FERNANDEZ-YEPEZ 1948.

Ctenolucius GiLl 1861.

Luciocharax STEINDACHNER 1876 : syn. de Ctenolucius G. 1861.

Spixostoma WhITLEY 1951.

Xiphostoma Agassiz 1829.

Xiphostoma SPIX 1829: pré-employé, syn. de Spixostoma W. 1951.

FAMILLE DES ANOSTOMIDAE

$(=$ Curimatidae $=$ Anodontidae $)$

Abramites Fowler 1906: syn. de Leporinus S. 1829.

Acuticurimata FOWLER 1941.

Allenina FeRnandez-YePEZ 1948. Anastomus MINDIN g 1832: syn. d'Anostomus (G.) S. 1777.

Anodus SPIX 1829.

Anostomoides Pellegrin 1908.

Anostomos Gronow 1763 : non valide.

Anostomus (G.) Scopoli 1777.

Anostomus (CUVIER) OKEN 1817: syn. de Leporinus S. 1829.

Atomaster EIgENMANN et Myers 1927.

Bondia Fernandez-YePez 1948 : préemployé, syn. de Bondichthys W. 1953.

Bondichthys WHITLEY 1953.

Caenotropus GüNTHER 1864. 
Camposella FeRnANDEZ-YePEZ 1948 : pré-employé, syn. de Stupens W. 1954.

Camposichthys WhItLeY 1953: préemployé, syn. de Stupens W. 1954.

- hilodus Müller et Troschel 1844: pré-employé, syn. de Caenotropus G. 1864.

Chilomyzon Fowler 1906: syn. de Prochilodus A. 1829.

Cruxentina Fernandez-Yepez 1948.

Curimata Walbaum 1792.

Curimatella EIGENMANN et EIGENMANN 1889.

Curimatichthys FERNANDEZ - YEPEZ 1948.

Curimatopsis STEINDACHNER 1876.

Curimatorbis FERNANDEZ-YEPEZ 1948.

Curimatus (CUVIER) OKen 1817 : syn. de Curimata W. 1792.

Eigenmannina FOWLER 1906.

Elopomorphus GILL 1878.

Garmanina FOWLER 1906.

Gnathodolus MYERS 1927.

Hamatichthys FERNANDEZ-YEPEZ 1948.

Hemicurimata MYERS 1929: s./g. de Curimata W. 1792.

Histiodromus GISTEL 1848 : syn. d'Anostomus (G.) S. 1777.

Hypomasticus BoRODIN 1929: s./g. de Leporinus S. 1829.

Ichthyoelephas.

Laemolyta COPE 1872.

Lahiliella EIGENMANN 1903.

Lambepiedra FERNANDEZ-YEPEZ 1948.

Lepipinna FERNANDEZ-YEPEZ 1948.

Leporellus LÜTKEN 1874.

Leporinodus EIGENMANN 1922.

Leporinus SPIX 1829.

Microdus KNER 1859: pré-employé, syn. de Caenotropus G. 1864. Mormyrhynchus SwaINSON 1839 : syn. de Anostomus (G.) S. 1777.
Pacu SpIX 1829.

Peltapleura Fowler 1906: syn. de Curimata W. 1792.

Pithecocharax Fowler 1906: syn. d'Anostomus (G.) S. 1777.

Potamorhina COPE 1878.

Prochilodus Agassiz 1829.

Psectrogaster EIGENMANN et EIGENMANN 1889.

Pseudocurimata FERNANDEZ-YEPEZ 1948.

Pseudopsectrogaster FERNANDEZYEPEZ 1948.

Rhytiodus KNER 1859.

Rivasella FERNANDEZ-YEPEZ 1948.

Sartor MYers et DE CARVAlho 1959.

Schizodon A G A SIZ 1829: syn. d'Anostomus (G.) S. 1777.

Schizodontopsis GARMAN 1890.

Semaprochilodus FOWLER 1941.

Semelcarinata FERNANDEZ-YEPEZ 1948.

Semitapiscis EIGENMANN et EIGENMANN 1889.

Steindachnerina FowLER 1906: syn. de Curimata W. 1792.

Steindachnerina FERNANDEZ-YEPEZ 1948: s./g. de Curimata W. 1792.

Stupens WhItLey 1954.

Suprasinelepichthys FERNANDEZYEPEZ 1948.

Synaptolaemus MYERS 1950.

Walbaumina FERNANDEZ-YEPEZ 1948.

Xyrocharax FOWLER 1913.

FAM. DES HEMIODONTIDAE

$$
\text { (= Hemiodidae) }
$$

Anisistia EIgenmanN 1903.

Apareiodon Eigenmann 1916.

Archicheir EIGENMANN 1909.

Centrophorus KNER 1859: pré-employé, syn. de Pareiodon.

Characidium REINHARDT 1866.

Chorimycterus COPE 1894: syn. de Characidium R. 1866. 
Hemiodopsis FOWLER 1906.

Hemiodus MüLLER 1843.

Jobertina Pellegrin 1909

Microcharax EIGENMANN 1909.

Nannobrycon HoEDEMAN 1950.

Nannostomus GüNTHER 1872.

Nanognathus BOULENGER 1895: syn. de Characidium R. 1866.

Nematoparodon FOWLER 1944: s./g. de Parodon C. et V. 1849.

Pareiodon KNERR 1855 : syn. de Parodon C. et V. 1849.

Parodon Cuvier et VAlenciennes 1849.

Parodontops Schultz et Miles 1943.

Poecilobrycon EigenManN 1909: syn. de Nannostomus G. 1872.

Poecilosomatops FowLER 1906: syn. de Characidium R. 1866.

Pterohemiodus FoWLER 1940.

Saccodon KNER 1863.

\section{FAMILLE DES CITHARINIDAE}

Belonophago Giltay 1929.

Citharidium BOULENGER 1902.

Citharinus (CUVIER) OKEN 1817.

Cytharinus OKEN 1817: errat. pro Citharinus (C.) O. 1817.

Distichodina FOWLER 1935: s./g. de Distichodus M. et T. 1845 .

Distichodomicrura FowLER 1936: s./g. de Distichodus M. et T. 1845.

Distichodura FOWLER 1936: s./g. de Distichodus M. et T. 1845 .

Distichodus Müller et Troschel 1845 .

Eugnathichthys BOULENGER 1898.

Gavialocharax PELLEGRIN 1926.

Hemistichodus PELLEGRIN 1900.

Ichthyoborus GüNTHER 1864.

Lepinannocharax FOWLER 1936: s./g. de Nannocharax G. 1867.

Mesoborus Pellegrin 1900.

Microdistichodus PELLEGRIN 1926.
Microstomatichthyoborus NicHOLS et GRISCOM 1917.

Monostichodus VaILlant 1900: nom. nud., syn. d'Hemistichodus P. 1900 .

Nannaethiops GüNTHER 1871.

Nannocharax GüNTHER 1867.

Neoborus BOULENGER 1898: pré-employé, syn. de Phagoborus M. 1924.

Neolebias SteIndachner 1894.

Paradistichodus PeLlegrin 1922.

Paraphago BOULENGER 1898.

Phago GüNTHER 1875.

Phagoborus MYERS 1924.

Ra WhITLEY 1931: syn. d'Ichthyoborus G. 1864.

Rhabdaethiops FOWLER 1936: s./g. de Nannaethiops G. 1871.

Thrissocharax MYERS 1926.

Tristichodus BOULENGER 1920: errat. pro Distichodus M. et T. 1845.

Xenocharax GÜNTHER 1867.

FAMILLE DES

RHAMPHICHTHYIDAE

Altona (KAUP) DuMÉRIL 1856: syn. de Rhamphichthys $M$. et T. 1849.

Brachyrhamphichthys GüNTHER 1870: syn. d'Hypopomus G. 1864.

Gymnorhamphichthys EIGENMANN 1912.

Hypopomus GILL 1864.

Rhamphichthys MüLLER et TROSCHEL 1849.

Steatogenys BOULENGER 1898.

FAMILLE DES GYMNOTIDAE

(Gymnotes, Carapos)

Carapo OKen 1817 : syn. de Gymnotus L. 1758.

Carapus (Cuvier 1817) Müller et Troschel 1849: syn. de Gymnotus L. 1758. 
Cryptops EigenmanN 1894: pré-employé, syn. de Eigenmannia J. et E. 1896.

Eigenmannia JoRdan et EVERManN 1896.

Giton (KAup) DumérIL 1856 : syn. de Gymnotus L. 1758.

Gymnotes GILl 1864 : syn. de Sternopygus $M$. et T. 1849 .

Gymnotus LINNÉ 1758.

Oedemognathus MYERS 1936.

Sternopygus Müller et Troschel 1849.

FAM. DES STERNARCHIDAE

(= Apteronotidae)

Apteronotus DE LACÉPÈde 1800 : syn. de Sternarchus B. et S. 1801.

Carapus RAFINESQUE 1810: pré-employé, syn. de Sternarchus B. et S. 1801 .

Memarchus Kaup 1856: syn. de Sternarchus B. et S. 1801.

Odontosternarchus ELLIS 1913.

Orthosternarchus ELLIS 1913.

Porotergus Eigenmann 1912.

Rabdolichops EIgENMANN et AlLEN 1942.

Rhamphosternarchus GüNTHER 1870 : syn. de Sternarchorhynchus C. 1855 .

Sternarchella EIGENMANN et WARD 1905.

Sternarchogiton EIGENMANN et WARD 1905.

Sternarchorhamphus EIGENMANN et WARD 1905.

Sternarchorhynchus DE CASTELNAU 1855.

Sternarchus BLOCH et SCHNEIDER 1801.

Tateichthys LAMONTE 1929.

Ubidia MILES 1945.

Uramara RIBEIRO 1922.
FAM. DES ELECTROPHORIDAE

(Anguille électrique ; Electric-eel)

Electrophorus GILL 1864.

Gymnotus LINNÉ 1766: syn. d'Electrophorus G. 1864.

\section{Sous-ordre des CYPRINOIDEI}

\section{FAM. DES CATOSTOMIDAE}

(Suckers or Buffalo-fishes)

Acomus Girard 1856: syn. de Catostomus S. 1817.

Amblodon RAFINESQue 1819: syn. d'Ictiobus R. 1820.

Bubalichthys AGASSIZ 1855: syn. d'Ictiobus R. 1820.

Carpiodes RAFINESQUe 1820.

Catostomus Le SueUr 1817.

Chasmistes JORDAN 1878.

Cycleptus RAFINESQUe 1819.

Decadactylus RAFINESQUE 1820 : syn. de Catostomus S. 1817.

Deltistes Seale 1896.

Erimyzon JoRDAN 1876.

Eurystomus RAFINESQUE 1820: non valide.

Hylomyzon Agassiz 1855 : syn. d'Hypentelium R. 1818.

Hypentelium RAFINESQUE 1818.

Ichthyobus AGASsIZ 1846: emend. pro Ictiobus R. 1820.

Ictiobus RAFINESQUE 1820.

Lagochila JORDAN et BRAYTON 1877 : pré-employé, syn. de Quassilabia J. et B. 1878 .

Lipomyzon COPE 1881.

Megapharynx LEGENDRE 1942.

Megastomatobus FowLeR 1913: syn. de Ictiobus R. 1820.

Minomus GIRARd 1856: syn. de Catostomus S. 1817.

Minytrema JoRDAN 1878.

Moxostoma RAFINESQUE 1820.

Myxocyprinus GILL 1878.

Myxostoma: vide Moxostoma R. 1820. 
Notolepidomyzon FOWLER 1913.

Pantosteus COPE 1876.

Pithecomyzon FowLER 1913.

Placopharynx COPE 1870.

Ptychostomus Agassiz 1855: syn. de Moxostoma R. 1820.

Quassilabia JORDAN et BRAYTON 1878 .

Scartomyzon FOWLER 1913: s./g. de Moxostoma R. 1820.

Sclerognathus CUvier et VALENCIENNES 1844: syn. de Moxostoma R. 1820 .

Stomocatus BonAPARTE 1840: syn. de Catostomus S. 1817.

Teretulus RAFINESQUE 1820.

Thoburnia JORDAN et SNYDER 1917. Xyrauchen KIRSCH 1888.

\section{FAMILLE DES CYPRINIDAE}

(Carpes, vairons et poissons blancs; Carps, minnows, Chubs and Daces) Abbottina JoRdAN et Fowler 1903. Abramidopsis Siebold 1863 : validité douteuse.

Abramis CUVIER 1817.

Abramocephalus STEINDACHNER 1869: syn. d'Hypophthalmichthys B. 1859.

Abrostomus SMITH 1849.

Acahara Jordan et HubBs 1925.

Acanthalburnus BERG 1916.

Acanthobrama HeCKel 1842.

Acanthobrama BLEEKER 1859.

Acanthobrama BLEEKER 1865.

Acanthogobio HeRZENSTEIN 1892 : syn. d'Hemibarbus B. 1859.

Acanthonotus (TICKELL) DAY 1888: pré-employé, syn. de Matsya D. 1889.

Acanthorhodeus BLEEKER 1870.

Acanthorutilus BERG 1912.

Acapoeta Cockerell 1910: s./g. de Varicorhinus R. 1836.

Acheilognathus BLEEKER 1869.

Acra BleEker 1863.

Acrocheilichthys BLEEKER 1869.
Acrocheilus Agassiz 1855.

Acrossochilus Oshima 1919.

Adamacypris FOWLER 1934: s./g. de Barbus C. et C. 1817.

Ageneiogarra GaRMAN 1912.

Ageniogobio SaUvage 1878.

Agosia GIRARD 1856.

Albulichthys BLEEKER 1859.

Alburnellus GIRARD 1856: syn. de Notropis R. 1818.

Alburnoides JeITteles 1861.

Alburnops GIRARD 1856.

Alburnus RAFINESQUE 1820.

Algansea GIRARD 1856.

Algoma GiRARD 1858: syn. d'Hybognathus A. 1855.

Allochela Silas 1958: s./g. de Chela H. 1822.

Allodanio Sмiтн 1945: s./g. de Danio H. 1822.

Altigena LIN 1933: s./g. d'Osteochilus G. 1868.

Amblypharyngodon BLEEKER 1859.

Amblyrhynchichthys B LEEKER 1859.

Amplolabrius LIN 1933.

Anabarilius.

Anchovicypris FOWLER 1936: s./g. d'Engraulicypris G. 1893.

Anematichthys BLEEKER 1859.

Aphyocypris GüNTHER 1868.

Aphyocyproides TANG 1942.

Apiorhynchus NIKOLSKY 1897: syn. de Schirothorax H. 1838.

Apiostoma NIKOLSKY 1897 : syn. de Schizothorax H. 1838.

Apocope COPE 1872.

Argyreus Heckel 1842.

Aristichthys OsHIMA 1919.

Armatogobio TARANETZ 1937.

Aspidoparia Heckel 1843.

Aspiobarbus BERG 1932.

Aspiolucius BERG 1907.

Aspiopsis Zugmayer 1912.

Aspiorhynchus KeSSLER 1879.

Aspius Agassiz 1835. 
Atrilinea CHU 1935.

Aturius Dubalen 1878 : syn. de Leuciscus C. 1817.

Aulopyge Heckel 1841.

Azteca Jordan et Evermann 1896: pré-employé, syn. d'Aztecula J. et E. 1898 .

Aztecula Jordan et Evermann 1898.

Balantiocheilos BLEEKER 1859.

Ballerus Heckel 1842.

Bangana HAMILTON 1822.

Barbellion WHITLEY 1931.

Barbichthys BLEEKER 1859.

Barbodes BLEEKER 1859: s./g. de Barbus C. et C. 1817.

Barbodon DyBowSKI 1872: syn. de Sarcocheilichthys B. 1869.

Barbopsis CAPORIACCO 1926.

Barbus Cuvier et Cloquet 1817.

Barilius HaMILTON 1822.

Barynotus GüNTHER 1868: pré-employé, syn. de Barbellion W. 1931.

Bathystoma FitzINGER 1873 : syn. de Telestes B. 1837.

Beirabarbus HERRE 1936.

Belligobio JoRdAN et HuBBs 1926.

Bendilisis BLEEKER 1859: syn. de Barilius H. 1822.

Bengala GRAY 1833.

Bertinichthys WHITLEY 1953.

Bertinius FANG 1943: pré-employé, syn. de Bertinichthys W. 1953.

Biwia JoRDAN et Fowler 1903.

Blicca HECKEL 1858.

Bliccopsis SIEBOLD 1863 : validité douteuse.

Bola GüNTHER 1868: pré-employé, syn. de Raiamas J. 1919.

Brachydanio WEBER et BEAUFORT 1916: s./g. de Danio H. 1822.

Brachygramma DAY 1865: syn. d'Amblypharyngodon B. 1859.

Brama KLEIN 1775: non valide, syn. d'Abramis C. 1817.

Brama (K.) BLEEKER 1862 : syn. d'Abramis C. 1817.

Brevigobio TANAKA 1916.
Bungia KeYSERIING 1861.

Cabdio Hamilton 1822.

Cachia JoRdAN 1917 : syn. de Chela H. 1822 .

Cachius GüNTHER 1868 : syn. de Chela H. 1822.

Caecobarbus Boulenger 1921.

Campostoma Agassiz 1855.

Candidia JORDAN et RICHARDSON 1909.

Capoeta Cuvier et VAlenciennes 1842: s./g. de Barbus C. et C. 1817.

Capoetobrama BERG 1916.

Caraspius NichOLS 1925.

Carassioides Oshima 1926: hybride.

Carassius NILsson 1832.

Carpio S. D. W. 1837 : syn. de $\boldsymbol{C y}$ prinus L. 1758.

Carpio HeCKel 1856: hybride.

Carpionichthys BLEEKER 1863: syn. de Cyprinus L. 1758.

Catla Cuvier et VAlenciennes 1844: syn. de Gibelion H. 1842.

Catlocarpio BOULENGER 1898.

Cenisophius Bonaparte 1845: syn. de Gardonus B. 1845

Cephalakompsus HeRRE 1924.

Cephalopsis FITZINGER 1873 : syn. de Leuciscus C. 1817.

Cephalus BONAPARTE 1845 : pré-employé, syn. de Leuciscus C. 1817.

Cephalus BASILEWSKI 1855 : pré-employé, syn. d'Hypophthalmichthys B. 1859.

Ceratichthys BAIRD et GIRARD 1853. Ceratichthys GIRARD 1856: syn. de Nocomis G. 1856.

Ceraticthys: errat. pro Ceratichthys B. et G. 1853 .

Chagunius Sмттн 1938.

Chalcalburnus BERG 1932.

Chanodichthys BLEEKER 1859.

Chedrus SwaInson 1839: syn. de Barilius H. 1822.

Cheilobarbus SMITH 1849.

Cheilonemus BAIRD 1855.

Chela Hamiton 1822. 
Chelaethiops BOULENGER 1899.

Cheonda GIRARD 1856: syn. de $\boldsymbol{T} \boldsymbol{i}$ goma G. 1856.

Chilogobio BERG 1914.

Chilogobio BERG 1915.

Chondrochilus HeCKel 1842 : syn. de Chondrostoma A. 1835.

Chondrorhynchus HECKEL 1842 : syn. de Chondrostoma A. 1835.

Chondrostoma Agassiz 1835.

Chriope JoRDAN 1878.

Chrosomus RAFINESQUE 1820.

Chrysophekadion BLEEKER 1859 : syn. de Labeo C. 1817 et de Morulius H. 1822.

Chuanchia HeRZENSTEIN 1888.

Cirrhina Cuvier 1817: syn. de Cirrhinus (C.) O. 1817.

Cirrhinichthys BLEEKER 1863.

Cirrhinus (C.) OKEN 1817.

Clarkina JORDAN et EVERMANN 1927.

Clinostomus GIRARD 1856: s./g. de Notropis R. 1818.

Cliola GIRARD 1856: syn. de Ceratichthys B. et G. 1853 .

Clypeobarbus FOWLER 1936: s./g. de Barbus C. et C. 1817.

Coccogenia Cockerell et Callaway 1909: syn. de Coccotis J. 1882.

Coccotis Jordan 1882.

Cochlognathus BAIRD et GIRARD 1854.

Codoma Girard 1856.

Coliscus Cope 1872: syn. de Pimephales R. 1820.

Coptostomabarbus DAVIDE et POLL 1937.

Coreius Jordan et Starks 1905.

Coreoleuciscus MORI 1935.

Coripareius GARMAN 1912.

Cosmochilus SaUVAge 1878.

Cosmochilus Sauvage 1882.

Couesius JORDAN 1878.

Crossocheilichthys BLEEKER 1863

Crossocheilus VAN HASSELT 1823

Ctenopharyngodon STEINDACHNER 1866.
Culter Basilewsky 1855: syn. de Pelecus A. 1835.

Culticula Aввотт 1901.

Cultrichthys SMITH 1938.

Cultriculus Osнiмa 1919.

Cultrops SмIтH 1938.

Cyclocheilichthys BLEEKER 1859.

Cyprinella GIRARD 1856.

Cyprinion HECKEL 1842.

Cyprinopsis BONAPARTE 1837: syn. de Carassius N. 1832.

Cyprinopsis Blanchard 1866: syn. de Carassius N. 1832.

Cyprinus LINNÉ 1758.

Cyrene Heckel 1843: syn. de Tor G. 1833 .

Dangilia JORDAN 1919: errat. pro Danglia C. et V. 1842.

Danglia Cuvier et VALEnCIENNES 1842: syn. de Tor G. 1833.

Danio Hamilton-Buchanan 1822.

Danioides CHU 1935.

Daniops SмIтн 1945.

Devario Bleeker 1859: syn. de Danio H. 1822.

Dillonia Heckel 1842: s./g. de Varicorhinus R. 1836.

Dionda GIRARD 1856.

Diplocheilichthys BLEEKER 1859.

Diplocheilus Van Hasselt 1823.

Diptychus STEINDACHNER 1866.

Discognathichthys BLEEKER 1859 : syn. de Garra H. 1822.

Discognathus Heckel 1842 : syn. de Garra H. 1822.

Discogobio SHU-YEN 1931.

Discolabeo FOWLER 1937.

Distoechodon PETERS 1880.

Dobula RAFInesque 1820: syn. de Leuciscus C. 1817.

Eillichthys Pellegrin 1929.

Eirmotus SchUltz 1959.

Elopichthys BLEEKER 1859.

Englottogaster GiSTEL 1848: syn. de Schizothorax H. 1838.

Engraulicypris GÜNTHER 1893.

Enteromius COPE 1867: s./g. de Barbus C. et C. 1817. 
Epalzeorhynchus BLEEKER 1855.

Episema COPE et JORDAN 1877 : préemployé, syn. de Paranotropis F. 1904.

Eremichthys HubBs et Miller 1948.

Ericymba COPE 1865.

Erimonax JORDAN 1924.

Erimystax JORDAN 1882.

Erinemus JORDAN 1876: syn. d'Hybopsis A. 1854.

Eritrema Cope 1876 : syn. d'Apocope C. 1872 .

Erogala Jordan et BRAYTON 1878. Erythroculter: syn. de Chanodichthys B. 1859.

Esomus Swainson 1839.

Estevea Whitley 1953: s./g. de Barbus C. et C. 1817.

Eucirrichthys PERUGIA 1892.

Eustira GüNTHER 1868: syn. de Danio H. 1822.

Evarra Woolman 1895.

Exoglossops Fowler et BEAN 1920.

Exoglossum RAFINESQUE 1818.

Extrarius JORDAN 1919.

Falcula JORDAN et SNYDER 1899: pré-employé, syn. de Falcularius J. et S. 1903.

Falcularius JORDAN et SNYDER 1903. Filirasbora FOWLER 1937.

Fusiana Jordan et STARKs 1905: syn. d'Aphyocypris G. 1868.

Gardonus BONAPARTE 1845.

Garra Hamilton-Buchanan 1822.

Georgichthys NichoLs 1918.

Gibelion HECKEL 1842.

Gila BAIRD et GIRARD 1853.

Glabrobarbus FOWLER 1931: s./g. de Barbus C. et C. 1817.

Gnathopogon BLEEKER 1859.

Gobio CUVIER 1817.

Gobiobarbus DYвоWSKI 1869: syn. d'Hemibarbus B. 1859.

Gobionichthys BLEEKER 1863.

Gobiosoma DYвowsKI 1872: syn. de Saurogobio B. 1870.
Gonoproktopterus JoRDAN 1919: errat. pro Gonoproktopterys B. 1859.

Gonoproktopterys BLEEKER 1859 : syn. d'Hypselobarbus B. 1859.

Gonorhynchus MC Clelland 1839: syn. de Crossocheilus H. 1823.

Grandisquamachela FOWLER 1934 : s./g. de Parachela S. 1881.

Graodus GüNTHeR 1868: syn. d'Alburnops G. 1856.

Gymnocypris GüNTHER 1868.

Gymnodiptychus HERZENSTEIN 1892.

Gymnognathus SAUVAGE 1884 : préemployé, syn. d'Aspidolucius B. 1907.

Gymnostomus HECKEL 1842: s./g. de Varicorhinus R. 1836.

Gyrinochilus VAILLANT 1902: vide Gyrinocheilus V. 1902, p. 55.

Habrolepis FITZINGER 1873: syn. de Telestes B. 1837.

Hainania KOLLER 1926.

Hampala Van Hasselt 1823.

Heegerius: vide Hegerius B. 1845.

Hegerius BONAPARTE 1845.

Hemibarboides WANG 1935.

Hemibarbus BLEEKER 1859.

Hemiculter BLEEKER 1859.

Hemiculterella VARPAKHOVSKY 1887.

Hemigrammocapoeta PELLEGRIN 1927.

Hemigrammocapoeta EsTÈVE 1952: pré-employé, syn. d'Estevea W. 1953.

Hemigrammocypris FOWLER 1910. Hemigrammopuntius PELLEGRIN 1923 : s./g. de Barbus C. et C. 1817.

Hemiplus RAFINESQUe 1820: syn. de Notemigonus R. 1819.

Hemitremia COPE 1870.

Henicorhynchus SMITH 1945.

Herzensteinia CHU 1935. 
Hesperoleucus SNYDER 1912.

Heteroleuciscus SAUVAge 1874.

Hingobio FANG 1938.

Horadandia Deraniyagala 1943.

Horalabiosa SILAS 1954.

Hudsonius GIRARD 1856: s./g. de Notropis R. 1818.

Hybognathus Agassiz 1855.

Hybopsis Agassiz 1854.

Hyborhynchus Agassiz 1855.

Hydrophlox JORDAN 1878.

Hylotylognathus FOWLER 1934.

Hypargyrus ForBes 1884: syn. de Ceratichthys B. et G. 1853.

Hypophthalmichthys BLEEKER 1859.

Hypselobarbus BLEEKER 1859.

Hypsilepis Cope 1864: vide Hypsolepis B. 1850.

Hypsolepis (BAIRD) Agassiz 1850: syn. de Luxilus R. 1820.

Iaus BLEEKER 1859: errat. pro Idus H. 1858.

Idus HeCKel 1858: s./g. de Leuciscus C. 1817 .

Incisilabeo FOWLER 1938: s./g. de Labeo C. 1817.

Iotichthys JORDAN et EVERMA N N 1896.

Iranocypris BRUUN et KAISER 1950.

Ischikaunia JORDAN et SNYDER 1900.

Isocephalus (HECKEL) BLEEKER 1863: syn. de Bangana H. 1822.

Kantaka HORA 1942: s./g. d'Osteochilus G. 1868.

Kendallia Evermann et Shaw 1927.

Klamatella MiLleR 1945: s./g. de Gila B. et G. 1853.

Labeo CUVIER 1817.

Labeobarbus RüPPELL 1836: syn. de Tor G. 1833.

Labeobarbus BLEEKER 1859: syn. de Tor G. 1833.

Labiobarbus : errat. pro Labeobarbus R. 1836.

Ladislavia DYBOWSKI 1869.

Lagowskiella DYвоWSKI 1916: syn. de Phoxinus R. 1820.
Lanceabarbus FOWLER 1936: s./g. de Barbus C. et C. 1817.

Laubuca BLEEKER 1859.

Lavinia BAIRD et GIRARD 1854.

Lepidocephalus BLEEKER 1858.

Lepidopygopsis RAJ 1941.

Leptobarbus BLEEKER 1859.

Leptobarbus BLEEKER 1863.

Leptocephalus BASILEWSKI 1855 : préemployé, syn. de Chanodichthys B. 1859.

Leptocypris BOULENGER 1900.

Leucalburnus BERG 1914.

Leucalburnus BERG 1916.

Leucaspis HECKEL et KNER 1858: vide Leucaspius $\mathrm{H}$. et K. 1858.

Leucaspius HECKEL et KNER 1858.

Leucidius SNYDER 1917.

Leucisculus OshIMA 1920.

Leuciscus KLEIN 1775: non valide.

Leuciscus CUVIER 1817.

Leucogobio GüNTHER 1896: syn. de Gnathopogon B. 1859.

Leucos Heckel 1843: syn. de Gardonus B. 1845.

Leucosoma: vide Leucosomus $\mathrm{H}$. 1842.

Leucosomus HecKel 1842: syn. de Cheilonemus B. 1855.

Liparus Schulze 1889: syn. de Rutilus R. 1820.

Lissocheilichthys OsHIMA 1920.

Lissochilus WEBER et BEAUFORT 1916.

Lissorhynchus BLEEKER 1859: syn. de Garra H. 1822.

Lobocheilus VAN HASSELT 1823 : syn. de Bangana H. 1822.

Longiculter FOWLER 1937.

Longurio JORDAN et STARKS 1905: pré-employé, syn. de Saurogobio B. 1878.

Luciobarbus HECKEL 1842.

Luciobrama BLEEKER 1870.

Luciocyprinus VAILLANT 1904.

Luciosoma BLEEKER 1855.

Luxilinus JoRDAN 1885: syn. de Lavinia B. et G. 1854.

ANN. PARASITO. HUM. ET COMP., 1962, 37, $\mathrm{n}^{\circ} 6^{\mathrm{b} / \mathrm{s}}$ (fasc. suppl.) 
Luxilus RAFINESQUE 1820: s./g. de Notropis R. 1818.

Luxinus RAFINESQUE 1820: vide Luxilus R. 1820.

Lythrurus JORDAN 1876.

Machaerochilus FITZINGER 1873 : syn. de Chondrostoma A. 1835.

Macrhybopsis COCKERELL et ALLISON 1909.

Macrochirichthys BLEEKER 1859.

Mandibulacra HERRE 1924.

Mannichthys SchULTZ 1942.

Margariscus COCKerell 1909.

Masticobarbus TANG 1942.

Matsya DAY 1889 : syn. de Mystacoleucus G. 1868.

Maxillilingua RAFINESQUE 1818: syn. d'Exoglossum R. 1818.

Mayoa DAY 1869.

Mearnsella SEALE et BEAN 1908.

Megagobio KessLer 1876: syn. de Rhinogobio B. 1870.

Megalobrama DYBOWSKI 1872: syn. de Parabramis B. 1865.

Megarasbora GüNTHER 1868: syn. de Bengala G. 1833.

Mekongina FOWLER 1937.

Mesocyprinus FENG 1946.

Mesocyprinus CHENG 1950.

Metallites Schulze 1889: syn. de Rutilus R. 1820.

Metzia JoRdAN et ThOMPSON 1914.

Micraspius DYвOWSKI 1869.

Microbarbus GÉRY 1958.

Microlepis Bonaparte 1845 : syn. de Leuciscus C. 1817.

Microphysogobio MORI 1934.

Microrasbora ANNANDALE 1918.

Miniellus Jordan 1888: syn. d'Alburnops G. 1856.

Minnilus RAFINESQUE 1820: syn. de Notropis R. 1818.

Moapa HubBs et Miller 1948.

Mola HeCKel 1843: pré-employé, syn. de Amblypharyngodon B. 1859.

Moniana GIRARD 1856: syn. de $\boldsymbol{C y}$ prinella B. 1856.
Morara Bleeker 1859: syn. d'Aspidoparia H. 1843.

Moroco Jordan et Hubbs 1925.

Morulius Hamilton-Buchanan 1822: s./g. de Labeo C. 1817.

Mrigala BleEker 1859: syn. de Cirrhinus (C.) O. 1817.

Mylocheilus AGASsIZ 1855.

Myloleuciscus GARMAN 1912.

Myloleucops CocKerell 1913: syn. de Tigoma G. 1856.

Myloleucus Cope 1872: syn. de Tigoma G. 1856.

Myloleucus GÜNTHER 1873.

Mylopharodon AYRES 1855.

Mylopharyngodon PETERS 1880.

Mystacoleucus GÜNTHER 1868.

Mystus KLEIN 1775: non valide.

Nandina Gray 1831.

Nasus BasIlewsKI 1855: syn. de Chondrostoma A. 1835.

Nazatexico WhITLEY 1931.

Nematabramis BOULENGER 1894.

Neobola VinciguerRa 1895.

Neocarassius de CASTELNAU 1872.

Neochela SiLAS 1958: s./g. de Chela H. 1822.

Neogastromyzon PoPTA 1905.

Neorohita FowLER 1937: s./g. d'Osteochilus G. 1868.

Neotylognathus : s./g. de Tylognathus H. 1842.

Nicholsiculter RENDAHL 1928.

Nicholsicypris CHU 1935.

Nicholsopuntius BERG 1932.

Nocomis GIRARD 1856.

Notemigonus RAFINESQUE 1819.

Notropis RAFINESQUE 1818.

Nukta Hora 1942: s./g. de Schismatorhynchos B. 1855 .

Nuria CUVIER et VALENCIENNES 1842: syn. d'Esomus S. 1839.

Ochetobius GüNTHER 1868.

Onychodon DYBOwSKI 1872 : syn. d'Hypophthalmichthys B. 1859.

Onychostoma GüNTHER 1896: s./g. de Varicorhinus R. 1836. 
Opistocheilus BLEEKER 1859: syn. de Schizothorax H. 1838.

Opsaridium PETERS 1855.

Opsaridium (P.) GüNTHER 1868 : syn. de Barilius H. 1822.

Opsariichthys BLEEKER 1863.

Opsarius Mc Clelland 1839: syn. de Barilius H. 1822.

Opsarius KNER 1865: pré-employé, syn. de Pseudorasbora B. 1859.

Opsopoea Jordan et EVERMANN 1896.

Opsopoeodus HaY 1880.

Orcella JoRDAN et EVERMANN 1896: pré-employé, syn. de Nazatexico W. 1931.

Orcula Jordan et EVERMANN 1900 : pré-employé, syn. de Nazatexico W. 1931.

Oregonichthys HuBBS 1929.

Oreichthys SMITH 1933.

Oreinus Mc Clelland 1839: syn. de Schizothorax H. 1838.

Oreoleuciscus VARPACHOWSKI 1899.

Oreonectes GÜNTHER 1868.

Orfus Fitzinger 1873: syn. de Gardonus B. 1845.

Orthodon GIRARD 1856.

Ortholeucos DERJAVIN 1938: s./g. de Rutilus R. 1820.

Ospatulus HeRRE 1924.

Osteobrama HeCKel 1842: syn. de Rohtee S. 1840.

Osteochilichthys HORA 1942: s./g. d'Osteochilus G. 1868.

Osteochilus GÜNTHER 1868.

Otakia Jordan et SNYDER 1900.

Owsianka DYвowsKI 1862: syn. de Leucaspius H. et K. 1858.

Oxybarbus VAILlant 1893.

Oxygaster VAN Hasselt 1823.

Oxygeneum FORBES 1885.

Pachychilon STEINDACHNER 1866.

Pachychilon SteIndachner 1882.

Pachystomus Heckel 1842 : pré-employé, syn. de Shacra B. 1859.

Papillocheilus SмITH 1943.

Parabarbus FRANZ 1910.
Parabarilius Pellegrin et FANG 1946.

Parabramis BLEEKER 1865.

Paracanthobrama BLEEKER 1865.

Paracheilognathus BLEEKER 1863.

Parachela STEINDACHNER 1881.

Paracrossocheilus POPTA 1904.

Paracrossochilus PoPTA 1902.

Paradanio (BLEEKER) DAY 1865 : syn. de Danio H. 1822.

Paralaubuca BLEEKER 1865.

Paraleucogobio BERG 1907.

Paranotropis FOWLER 1904.

Parapelecus GÜNTHER 1889.

Paraphoxinus BLEEKER 1863: syn. de Phoxinellus H. 1840.

Paraprocypris FANG 1946.

Parapsilorhynchus HORA 1921.

Pararasbora REgAN 1908.

Pararhodeus BERG 1907: s./g. de Phoxinellus H. 1840.

Pararutilus BERG 1912: s./g. de Rutilus R. 1820.

Paraschizothorax BLEEKER 1863.

Parasinilabeo Wu 1939.

Paratylognathus SaUVAge 1880.

Parexoglossum HuBBs 1931.

Parosteobrama TCHANg 1930.

Pelecus Agassiz 1835.

Peletrophus GüNTHER 1864.

Percocypris CHU 1935.

Perilampus Mc Clelland 1839: syn. de Barilius H. 1822.

Pfrille JoRDAN 1924.

Phenacobius COPE 1867.

Photogenis COPE 1867.

Phoxinellus HECKEL 1840

Phoxinus RAfINESQUE 1820.

Phoxinus Agassiz 1835: syn. de Phoxinus R. 1820.

Phoxiscus Oshima 1919.

Phreatichthys Vinciguerra 1924.

Pigus Bonaparte 1845 : syn. de Gar. donus B. 1845.

Pimephales Rafinesque 1820. 
Plagiognathops BERG 1907.

Plagiognathus DYBOWSKI 1872: préemployé, syn. de Plagiognathops B. 1907.

Plagopterus COPE 1874.

Plargyrus RAFINESQUE 1820.

Platycara Mc Clelland 1839: syn. de Garra H. 1822.

Platygobio GILL 1863.

Platypharodon HeRZENSTEIN 1888.

Pogonichthys GIRARD 1854.

Pogonocharax REgAN 1907 : syn. d'Esomus S. 1839.

Pogonotropis FowleR 1935: s./g. d'Alburnus R. 1820.

Poropuntius SMITH 1931.

Pristiodon DyвоwsкI 1877: syn. de Ctenopharyngodon S. 1866.

Probarbus Sauvage 1880.

Procypris LIN SHU YeN 1933.

Prolabeo Norman 1932.

Prolabeops Schultz 1941.

Protoporus COPE 1872: syn. de Tigoma G. 1856.

Pseudaspius DYBOWSKI 1869.

Pseudobarbus Sмттн 1849.

Pseudobrama BLEEKER 1870.

Pseudoculter BLEEKER 1869.

Pseudogobio BLEEKER 1859.

Pseudogobiops BERG 1914.

Pseudogyrinocheilus FANG 1933.

Pseudohemiculter Nichols et PoPE 1927: syn. de Hainania KoLLER 1926.

Pseudolaubuca BLEEKER 1865.

Pseudoperilampus BLEEKER 1863.

Pseudophoxinus BLEEKER 1859.

Pseudopungtungia MORI 1935.

Pseudorasbora BLEEKER 1859.

Pterocapoeta GüNTHER 1902: s./g. de Varicorhinus R. 1836.

Pteropsarion GüNTHER 1868.

Ptychidio MYers 1930.

Ptychobarbus STEINDACHNER 1866.

Ptychocheilus Agassiz 1855.

Pungtungia Herzenstein 1892.

Puntioplites SMITH 1929.
Puntius Hamilton-Buchanan 1822 :

s./g. de Barbus C. et C. 1817.

Racoma Mc Clelland 1842.

Raiamas JORDAN 1919.

Rambaibarnia FOWLER 1934: s./g. de Danio H. 1822.

Rasbora BLEEKER 1859.

Rasborella FOWLER et BEAN 1923.

Rasborichthys BLEEKER 1859.

Rasborinus Oshima 1920: syn. de Metzia.

Rastineobola FOWLER 1936: s./g. de Neobola V. 1895.

Rectoris LIN 1935.

Rhinichthys Agassiz 1848: syn. d'Argyreus H. 1842.

Rhinogobio BLEEKER 1870.

Rhinogobioides RENDAHL 1929.

Rhodeoides THOMINoт 1884.

Rhodeops FOWLER 1910.

Rhodeus Agassiz 1855.

Rhodopleuriscus FOWLER 1944.

Rhynchocypris GüNTHER 1889.

Richardsonius GIRARD 1856.

Rohanus CHU 1935.

Rohita Cuvier et VAlenciennes 1842 : syn. de Labeo C. 1817.

Rohitichthys BLEEKER 1859.

Rohitodes BLEEKER 1863.

Rohtee SYKES 1841.

Rohteichthys BLEEKER 1859.

Rostrogobio TARANETZ 1937.

Rubellus FITZINGER 1873: syn. de Rutilus R. 1820.

Rutilus RAFINESQUE 1820.

Sagittabarilius FOWLER 1936: s./g. de Barilius H. 1822.

Salmophasia SwaINSON 1839 : syn. de Salmostoma S. 1839.

Salmostoma SWAINSON 1839.

Sambiva ANNANDAle 1918.

Sanagia Holly 1926.

Sarcidium COPE 1872: syn. de Phenacobius C. 1867.

Sarcocheilichthys BLEEKER 1869.

Sarragia Holly 1927. 
Saurogobio BLEEKER 1870.

Scaphesthes OsHima 1919.

Scaphiodon HECKEL 1842: s./g. de Varicorhinus R. 1836.

Scaphiodonichthys VINCIGUERRA 1889.

Scaphiodontella OsHima 1920.

Scaphiodontopsis FOWLER 1934.

Scaphognathops SMITH 1945.

Scaphognathus SмITH 1931: pré-employé, syn. de Scaphognathops S. 1945.

Scardiniopsis JÁCKEL 1864: validité douteuse.

Scardinius BONAPARTE 1837.

Schacra GüNTHER 1868: vide Shacra G. 1859.

Schismatorhynchos BLEEKER 1855.

Schizocypris REGAN 1914.

Schizopyge HeCKEL 1843.

Schizopygopsis STEINDACHNER 1866.

Schizothorax HeCKEL 1838.

Scomberopsis: errat pro Scombrocypris G. 1889.

Scombrocypris GüNTHER 1889.

Securicula GüNTHER 1868: syn. de Salmostoma S. 1839.

Semiculter CHU 1935.

Semilabeo Peters 1880.

Semiplotus BLEEKER 1859.

Semotilus RAFINESQUE 1820.

Shacra BLEEKER 1859.

Siaja BLEEKER 1859.

Siboma GIRARD 1856: s./g. de Gila B. et G. 1853 .

Sikukia SмIтH 1931.

Sinibarbus Sauvage 1874.

Sinibrama WU 1939.

Sinigobio CHU 1935.

Sinilabeo RENDAHL 1933.

Sinocyclocheilus FANG 1946.

Siphateles COPE 1883.

Smiliogaster JoRDAN 1919: errat. pro Smilogaster B. 1859.

Smilogaster BLEEKER 1859: syn. de Rohtee S. 1841.

Snyderichthys MILLER 1945.
Spinibarbichthys Oshima 1926: syn. de Mystacoleucus G. 1868.

Spinibarbus Oshima 1919: syn. de Mystacoleucus G. 1868.

Spirlingus Fatio 1890 : syn. de Blicca H. 1858.

Spirlinus: errat. pro Spirlingus F. 1890.

Spratellicypris HERRE et MYERS 1931.

Squalalburnus BERG 1932.

Squalidus DyBowsKI 1872: syn. de Gnathopogon B. 1859.

Squaliobarbus GÜNTHER 1868.

Squalius BonAPARTE 1837: s./g. de Leuciscus C. 1817.

Stilbe DeKAY 1842: syn. de Notemigonus R. 1819.

Stilbius Gill 1865 : syn. de Notemigonus R. 1819.

Stypodon Garman 1881.

Symmetrurus JORDAN 1878: syn. de Pogonichthys G. 1854.

Systomus Mc Clelland 1839.

Tambra BLEEKER 1859.

Tanakia JORDAN et THOMPSON 1914.

Tanichthys LIN 1932.

Telestes BONAPARTE 1837.

Temeculina COCKERELL 1909: s./g. de Gila B. et G. 1853

Thynnichthyina FOWLER 1937 : s./g. de Thynnichthys B. 1859.

Thynnichthys BLEEKER 1859.

Tiaroga GIRARD 1856.

Tigoma GIRARD 1856.

Tinca CUVIER 1817.

Tirodon HAY 1882.

Tor GRAY 1833.

Toxabramis GüNTHER 1873.

Trachybrama HECKEL 1863: syn. d'Acanthobrama H. 1842.

Tribolodon SAUVAge 1883.

Tricherodon (ForBes) JoRDAN et GILBERT 1882: syn. d'Opsopoedus H. 1880 .

Trinematichthys BLEEKER 1859: syn. de Luciosoma B. 1855.

Tylognathus HeCKEL 1842: syn. de Bangana H. 1822 . 
Tylognathus GüNTHER 1868: syn. de Gymnostomus H. 1842.

Typhlogarra TraWAVAS 1955.

Vanderbiltella FOWLER 1935: s./g. de Barbus C. et C. 1816.

Varicogobio SHU-YEN 1931.

Varicorhinus RüPPELL 1836.

Vimba FITZINGER 1873.

Xenendum Jordan et SNYDER 1899.

Xenobarbus NoRMAN 1923.

Xenocheilichthys SMITH 1934.

Xenocypris GüNTHER 1868.

Xenopomichthys PELLEGRIN 1905.

Xystrosus Jordan et SNYDER 1899.

Yaoshanicus SHU-YEN 1931.

Yuriria Jordan et Evermann 1896.

Zacco Jordan et EvermanN 1902.

Zezera JORDAN et FOWLER 1903.

Zopa Fitzinger 1873: syn. de Ballerus H. 1842.

Zophendum JORDAN 1878: syn. d'Agosia G. 1856.

\section{FAMILLE DES MEDIDAE}

Lepidomeda COPE 1874.

Meda GIRARD 1856.

Plagopetrus COPE 1874.

\section{FAMILLE DES COBITIDAE}

(Loches; Loaches)

Aborichthys CHAUDHURI 1913.

Acanthocobitis Peters 1861.

Acanthophthalmus VAN HASSELT 1823.

Acanthopsis Van Hasselt 1823.

Acanthopsis Agassiz 1835: syn. de Cobitis L. 1758.

Acanthopsoides FOWLER 1934.

Acoura Swainson 1839: syn. de Nemacheilus H. 1823.

Acourus Swainson 1839: syn. de Nemacheilus H. 1823.

Aperioptus Richardson 1848.

Apua BLyTh 1860.

Barbatula LINCK 1790.
Botia Gray 1831.

Canthophrys Swainson 1839: syn. de Botia G. 1831.

Cobitichthys BLEEKER 1859: syn. de Misgurnus L. 1803.

Cobitinula HANKO 1925.

Cobitis LINNÉ 1758.

Cobitophis MYERS 1927.

Crossostoma Sauvage 1878.

Deuterophysa RENDAHL 1933: syn. de Didymophysa W. 1958.

Diacantha SwAINSON 1839: syn. de Botia G. 1831.

Didymophysa WhITLEY 1958.

Diplophysa KessLer 1874: syn. de Didymophysa W. 1958.

Diplophysoides FowLER 1959: syn. de Didymophysa W. 1958.

Elxis Jordan et Fowler 1903: syn. de Lefua H. 1888.

Enobarbichthys WHITLEY 1931.

Eonemachilus BERG 1938.

Gobiobotia KREYENBERG 1911.

Gobitis FLEMING 1828: errat. pro Cobitis L. 1758.

Hedinichthys RENDAHL 1933: s./g. de Nemacheilus Van Hasselt 1823.

Homatula Nichols 1925.

Hymenophysa GüNTHER 1839: emend. pro Hymenphysa M. C. 1839.

Hymenphysa Mc Clelland 1839 : syn. de Botia G. 1831

Lefua Herzenstein 1888.

Lepidocephalichthys BLEEKER 1863.

Leptobotia BLEEKER 1870.

Mesomisgurnus FANG 1935.

Misgurnus DE LACÉPÈdE 1803.

Modigliania Perugia 1893.

Neacanthopsis SMITH 1945.

Nemacheilus Van Hasselt 1823.

Nemachilichthys DAY 1878: s./g. de Nemacheilus H. 1823.

Nemachilus: vide Nemacheilus $\mathbf{H}$. 1823.

Neomacheilus : erratum. pro Nemacheilus H. 1823. 
Octonema MARTENS 1868: pré-employé, syn. de Lefua H. 1888.

Octonema HerzensteIn et VARPACHOVSKI 1887 : pré-employé, syn. de Lefua H. 1888.

Oreias Sauvage 1874: syn. de Barbatula L. 1790.

Orthrias Jordan et Fowler 1903 : syn. de Barbatula L. 1790.

Oxygaster VAN HASSELT 1823.

Pangio Blyth 1860: syn. d'Acanthophthalmus H. 1823.

Paracobitis BLEEKER 1863.

Paralepidocephalus TCHANG 1935.

Paramisgurnus SAUVAGE 1878.

Platacanthus DAY 1865 : syn. de Lepidocephalichthys B. 1863.

Pogononemacheilus

s./g. de Nemacheilus H. 1823.

Prostheacanthus BLYTH 1860: syn. d'Acanthopsis H. 1823.

Pseudodon KessLer 1874: pré-employé, syn. de Paracobitis B. 1863.

Pterozygus DE LA PyLAIE 1835: syn. de Cobitis L. 1758

Sabanejewia VLADYKOV 1929.

Schistura Mc Clelland 1839: syn. de Barbatula L. 1790.

Sinibotia FANG 1937: s./g. de Botia G. 1831 .

Somileptes Swainson 1839: syn. de Cobitis L. 1758.

Syncrossus Blyth 1860 : syn. de Botia G. 1831 .

Tauphysa RENDAHL 1933: s./g. de Nemacheilus H. 1823.

Triplophysa RENDAHL 1933: s./g. de Nemacheilus H. 1823.

Ussuria NiKolsKI 1904 : syn. de Misgurnus L. 1803.

Vaillantella FOWLER 1906.

Yunnanilus Nichols 1925: s./g. de Nemacheilus H. 1823.
FAM. DES HOMALOPTERIDAE

Annamia HORA 1932.

Balitora Gray 1833: syn. d'Homaloptera H. 1823.

Balitoropsis SмIтH 1945.

Beaufortia HORA 1932.

Bhavania HORA 1920.

Blavania HoRA 1922 : vide Bhavania H. 1920.

Chopraia PRASHAD et MUCKerJI 1929.

Ellopostoma Vaillant 1902.

Formosania Oshima 1919.

Glaniopsis BOULENGER 1899.

Gyrinocheilops FowLER 1937: syn. de Gyrinocheilus V. 1902.

Gyrinocheilus VAILLANT 1902.

Helgia Vinciguerra 1889.

Hemimyzon REGAN 1911.

Homaloptera Van Hasselt 1823.

Homalopterula FOWLER 1940.

Homalopteroides FOWLER 1905.

Lepturichthys REGAN 1911.

Limparhomaloptera FANG 1935.

Metahomaloptera CHANG 1944.

Neohomaloptera HERRE 1945: s./g. d'Homaloptera H. 1823.

Paraprotomyzon Pellegrin et FANG 1935.

Parhomaloptera VaIllant 1902.

Praeformosania FANG 1935.

Progastromyzon HoRA et JARAYAM 1952.

Protomyzon HoRA 1932.

Pseudogastromyzon NichOLS 1925 : s./g. d'Hemimyzon R. 1911.

Pseudohomaloptera Silas 1953.

Psilorhynchus Mc Clelland 1839: syn. de Homaloptera H. 1823.

Sewellia Hora 1932.

Sinogastromyzon FANG 1930.

Sinohomaloptera FANG 1930: s./g. d'Homaloptera H. 1823.

Travancoria HORA 1941.

Vanmanenia HORA 1932. 
FAMILLE DES ADIPOSIIDAE Adiposia AnNandale et Hora 1921.

\section{Sous-ordre des SILUROIDEI}

(Silures ou Poissons-chats ; Cat-fishes)

\section{FAMILLE DES DIPLOMYSTIDAE}

Diplomystax (DUMÉRIL) GÜNTHER 1862: emend. pro Diplomystes B. 1862 .

Diplomyste DumérIL 1856: non valide.

Diplomystes (D.) BLEEKER 1862.

FAMILLE DES ARIIDAE

(= Tachysuridae) (Sea Cat-fishes)

Aelurichthys Gill: syn. de Bagre (C.) O. 1817.

Ailurichthys BAIRD et GIRARD 1854 : syn. de Bagre (C.) O. 1817.

Ancharius STEINDACHNER 1880.

Anemanotus FowLER 1944 : s./g. de Bagre (C.) O. 1817.

Ariodes Müller et Troschel 1848. Ariopsis GILL 1861: syn. d'Hexanematichthys B. 1858.

Ariopsis PeYer 1928: syn. de Eopeyeria W. 1947.

Arius Cuvier et Valenciennes 1840. Aspistor Jordan et Evermann 1898. Bagre (CUVIER) OKEN 1817.

Batrachocephalus BLEEKER 1847.

Batrachocephalus BLEEKER 1858.

Breviceps SwaInson 1838: pré-employé, syn. de Bagre (C.) O. 1817.

Brustarius HERRE 1935: s./g. d'Arius C. et V. 1840.

Catastoma (KUHL et VAN Hasselt) BLEEKER 1862: non valide, syn. de Netuma B. 1858.

Cathorops Jordan et GILBERT 1882. Cephalocassis BLEEKER 1858.

Cinetodus OGILBy 1898.
Cochlefelis WhItLey 1941.

Doiichthys WEBER 1913.

Eopeyeria WhITLEX 1947.

Felichthys Swainson 1839: syn. de Bagre (C.) O. 1817.

Galeichthys CUVIER et VALENCIENNES 1840.

Genideus de CASTELNAU 1855.

Glanis Agassiz 1829.

Guiritinga BLEEKER 1858.

Hemiarius BLEEKER 1862.

Hemipimelodus BLEEKER 1858.

Hexanematichthys BLEEKER 1858.

Ketengus BLEEKER 1847.

Ketengus BLEEKER 1858.

Leptarius GILl 1863: syn. de Selenaspis B. 1858.

Mystus Gronow 1854: syn. de Bagre (C.) O. 1817.

Nedystoma OGILby 1898.

Nemapteryx OGILby 1908.

Neoarius de CASTelnau 1877.

Netuma BLEEKER 1858.

Notarius Gill 1863: syn. d'Arius C. et V. 1840.

Osteogeneiosus BLEEKER 1847.

Osteogeneiosus BLEEKER 1858.

Pachyula Ogil by 1898: syn. d'Hemipimelodus B. 1858 .

Paradiplomystax GüNTHER 1864 : emend. pro Paradiplomystes B. 1863.

Paradiplomystes BLEEKER 1863.

Parancistrus BLEEKER 1862.

Pararius Whitley 1940: s./g. d'Arius C. et V. 1840.

Peyeria Whitley 1940: syn. d'Eopeyeria W. 1947.

Pseudarius BLEEKER 1862: syn. d'Arius C. et V. 1840.

Sciadeichthys BLEEKER 1863 : syn. de Selenaspis B. 1858.

Sciadeops Fowler 1944: s./g. de Sciades.

Sciades Müller et Troschel 1849 : pré-employé, syn. de Selenaspis B. 1858 .

Selenaspis BLEEKER 1858. 
Septobranchus HARDENBERG 1945. Tachysurus DE LACÉPÈDE 1803 : syn. d'Arius C. et V. 1840.

Tetranesodon WEBER 1913.

\section{FAMILLE DES DORADIDAE}

Acanthodoras BLEEKER 1862.

Agamyxis COPE 1878.

Ageneiosus de LACÉPÈdE 1803.

Amblydoras BLEEKER 1862.

Anadoras Eigenmann 1925.

Apuredoras FernandeZ-YePEZ 1951.

Asterophysus KNER 1858.

Astrodoras BleEKer 1862: syn. de Centrochir A. 1829.

Auchenipterichthys BLEEKER 1862.

Auchenipterus CUVIER et VALENCIENNES 1840.

Autanadoras FernandeZ-YepeZ 1950.

Calaphractus CATESBY 1771: non valide.

Centrochir AgASSIZ 1829.

Centrodoras EIGENMANN 1925.

Centromochlus KNER 1859.

Ceratocheilus RIBEIRo 1918: pré-employé, syn. d'Osteomystax W. 1940.

Ceratorhynchus AgAssiz 1829: non valide.

Davalla BLEEKER 1858.

Dianema COPE 1872.

Doraops Schultz 1944.

Doras DE LACÉpède 1803.

Entomocorus EIGENMANN 1917.

Epapterus COPE 1878.

Euanemus Müller 1843 : syn. d'Auchenipterus C. et V. 1840.

Franciscodoras EIGENMANN 1925.

Glanidium LÜTKEN 1874.

Hemidoras BLEEKER 1858: syn. de Doras L. 1803.

Hoplodoras EIGENMANN 1925.

Hypodoras EIGENMANN 1925.

Leptodoras BOULENGER 1898.
Lithodoras BLEEKER 1862: syn. de Centrochir A. 1829.

Megalodoras EIGENMANN 1925.

Mormyrostoma RIBEIRO 1912.

Nemadoras EIGENMANN 1925.

Opsodoras EIGENMANN 1925.

Orinocodoras MYERS 1927.

Osteomystax WHITLEY 1940.

Oxydoras KNER 1855.

Parasturisoma RIBEIRO 1912.

Parauchenipterus BLEEKER 1862 : syn. de Trachycoristes B. 1858.

Physopyxis COPE 1872.

Platydoras BLEEKER 1862: syn. de Doras L. 1803.

Pseudageneiosus BLEEKER 1862.

Pseudauchenipterus BLEEKER 1862.

Pseudepapterus STEINDACHNER 1893.

Pseudepterus SteIndACHNeR 1915.

Pseudodoras BLEEKER 1858.

Pterodoras BLeEKer 1862: syn. de Centrochir A. 1829.

Rhinodoras BLEEKER 1862: syn. d'Oxydoras K. 1855.

Scorpiodoras EIGENMANN 1925.

Tannayia RIBEIRO 1918.

Tatia RIBEIRO 1912.

Tetranematichthys BLEEKER 1862.

Trachelyopterichthys BLEEKER 1862.

Trachelyopterus CUVIER et VALENCIENNES 1840.

Trachycorystes BLEEKER 1858.

Trachycorystes BLEEKER 1862.

Trachydoras EIGENMANN 1925.

Tympanopleura EIGENMANN 1912.

Wertheimeria SteINDACHNER 1876.

Zathorax COPE 1872.

\section{FAMILLE DES PLOTOSIDAE}

Anodontoglanis RENDAHL 1922.

Anyperistius Ogilby 1908: syn. de Tandanus M. 1839.

Cainosilurus MACLEAY 1881.

Choeroplotosus KNER 1864: syn. de Cnidoglanis G. 1864. 
Cnidoglanis GÜNTHER 1864.

Copidoglanis GüNTHER 1864: syn. de Tandanus M. 1839.

Copiodoglanis JoRDAN 1919: errat. pro Copidoglanis G. 1864.

Deportator GISTEL 1848: syn. de Plotosus L. 1803.

Endorrhis OgILby 1898: syn. de Paraplotosus B. 1862.

Euristhmus OGILBY 1899.

Exilichthys WHITLEY 1933.

Gastromyzon GüNTHER 1874.

Lambertia PERUGIA 1894: pré-employé, syn. de Lambertichthys W. 1938 .

Lambertichthys WHITLEY 1938.

Neoplotosus de CASTELnaU 1875.

Neosilurus DE CASTELNAU 1877 : préemployé, syn. de Cainosilurus M. 1881 .

Oloplotosus WEBER 1913.

Ostophycephalus OGILBY 1899: syn. de Cnidoglanis G. 1864.

Paraplotosus BLEEKER 1862.

Plotosus DE LACÉPÈdE 1803.

Porochilus WeBER 1913.

Tandanus MrTCHELl 1839.

\section{FAMILLE DES SILURIDAE}

(Glanes ; Sheat-fishes)

Apodoglanis FowLER 1905.

Belodonichthys BLEEKER 1858.

Callichrous HAMILTON-BUCHANAN

1822: syn. de Ompok L. 1803.

Centromochlus KNER 1858.

Ceratoglanis MYERS 1938.

Clarasilurus FOWLER 1937.

Conta Hora 1950.

Cryptopterus GüNTHER 1864: vide Kryptopterus B. 1858.

Diastatomycter VaILlant 1891 : syn. d'Hemisilurus B. 1858.

Erethistoides HORA 1950.

Eumeda DE CASTElnaU 1878: syn. de Neosilurus S. 1867.

Glanis Agassiz 1856: pré-employé, syn. de Parasilurus B. 1862.

Hemisilurus BLEEKER 1858.
Herklotsella HERRE 1934.

Hito HaIgG : syn. de Hitoich. thys H. 1926.

Hito Herre 1924.

Hitoichthys HERRE 1926.

Kryptopterichthys BLEEKER 1858.

Kryptopterus BLEEKER 1858.

Micronema BLEEKER 1858.

Neosilurus STEINDACHNER 1867.

Ompok DE LACÉPÈDE 1803.

Parasilurus BLEEKER 1862.

Penesilurus Herre 1924.

Phalacronotus BLEEKER 1858.

Pseudosilurus BLEEKER 1858: syn. de Ompok L. 1803.

Pterocryptis Peters 1861.

Silurichthys BLEEKER 1858.

Silurodes BLEEKER 1860.

Silurodon KNER 1864: syn. de Wallago B. 1851.

Silurus LINNÉ 1758.

Uegitglanis GIANFERRARI 1923.

Wallago BLEEKER 1851.

Wallagonia MYers 1938: syn. de Wallago B. 1851.

\section{FAMILLE DES BAGRIDAE}

Amarginops Nichols et Griscom 1917.

Aoria Jordan 1919: pré-employé, syn. de Mystus (G.) S. 1777.

Aorichthys Wu 1939: syn. de Mystus (G.) S. 1777.

Aspidobagrus BLEEKER 1862 : syn. de Mystus (G.) S. 1777.

Auchenaspis BLEEKER 1858: pré-employé, syn. d'Auchenoglanis G. 1864.

Auchenoglanis GüNTHER 1864.

Bagrichthys BLEEKER 1860.

Bagroides BLEEKER 1851.

Bagrus CuVIER et VALENCIENNES 1839.

Brachymystus FOWLER 1937: s./g. de Mystus (G.) S. 1777.

Branchiostegus GILL 1862. 
Chrysichthys BLEEKER 1858.

Chrysobagrus BOULENGER 1899: syn. de Chrysichthys B. 1858.

Clarotes KNER 1855.

Coreobagrus MORI 1935.

Cranoglanis Peters 1880.

Dermocassis Nichols 1925: s./g. de Leiocassis B. 1858.

Duxordia MYERS 1951.

Fluvidraco JORDAN et FowLer 1903 : syn. de Pelteobagrus B. 1865.

Gephyroglanis BOULENGER 1899.

Gnathobagrus Nichols et GRISCOM 1917.

Gogrius DAY 1867 : syn. de Rita B. 1858.

Hemibagrus BLEeKER 1862: syn. de Mystus (G.) S. 1777.

Heterobagrus BLEEKER 1864.

Hypselobagrus BLEEKER 1862: syn. de Mystus (G.) S. 1777.

Laguvia HoRA 1921.

Leiocassis BLEEKER 1858.

Leptoglanis BOULENGER 1902.

Liauchenoglanis BOULENGER 1916.

Liobagrus HILGENDORF 1878.

Liocassis GüNTHER 1864 : emend. pro Leiocassis B. 1858.

Lophiobagrus POLL 1942.

Macrones DuméRIL 1856: syn. de Mystus (G.) S. 1777.

Macronichthys WhITE et MoY-ThоMAS 1940 : syn. de Mystus (G.) S. 1777.

Macronoides HORA 1921.

Macropterobagrus NicHOLS 1925 : s./g. de Mystus (G.) S. 1777.

Melanodactylus BLEEKER 1858: syn. de Chrysichthys B. 1858.

Mystus Gronow 1763 : non valide.

Mystus (G.) Scopoli 1777.

Nasocassis Nichols 1925: s./g. de Leiocassis B. 1858.

Neobagrus BellotTI 1892: syn. de Liobagrus H. 1878.

Notoglanidium GïNTHER 1902.

Octonematichthys BLEEKER 1858: syn. de Clarotes K. 1855.

Olyra Mc Clelland 1842.
Osteobagrus JAYARAM 1955: s./g. de Mystus (G.) S. 1777.

Parauchenoglanis BOULENGER 1911.

Pelteobagrus BLEEKER 1865.

Phyllonemus BOULENGER 1906.

Porcus Geoffroy-St-Hillaire 1818 : syn. de Bagrus C. et V. 1839.

Pradiahipokia FOWLER 1934.

Pseudeutropichthys KOLLER 1926: syn. de Cranoglanis P. 1880.

Pseudobagrichthys BLEEKER 1862: syn. de Bagroides B. 1851.

Pseudobagrus BLEEKER 1858.

Pseudobagrus BLEEKER 1860.

Rama BleEKER 1858.

Rhinobagrus BLEEKER 1865: s./g. de Leiocassis B. 1858.

Rita BLEEKER 1858.

Speraia Holly 1939: syn. de Mystus (G.) S. 1777.

Trachysurus RAFINESQUE 1815.

\section{FAMII.LE DES AMEIURIDAE (Horned Pout, Channel cats)}

Ameiurus RAfINESQUE 1820.

Amiurus: errat. pro Ameiurus $\mathrm{R}$. 1820.

Bagre CATESBY 1771: non valide. Elliops RAFINESQue 1820: syn. d'Ictalurus R. 1820.

Gronias COPE 1864.

Haustor Jordan et EVERMANN 1896. Hopladelus GILL 1861: emend. pro Opladelus R. 1820.

Ichthaelurus: emend. pro Ictalurus R. 1829.

Ictalurus RAFINESQUE 1820.

Ilictis RAFINESQUE 1820 : syn. de Leptops R. 1820.

Istlarius JORDAN et SNYDER 1899.

Leptops RAFINESQUE 1820

Noturus RAFINESQUE 1818.

Opladelus RAFINESQUE 1820: syn. de Leptops R. 1820.

Pelodichthys JORDAN 1877: emend. pro Pilodictis R. 1819.

Pilodictis RAFINESQUE 1819: non valide. 
Prietella CARRANZA 1954.

Rabidus JORDAN et EVERMANN 1896.

Satan HubBs et BaIley 1947.

Schilbeodes BLEEKER 1858.

Synechoglanis GILL 1858: syn. d'Ictalurus R. 1820.

Trogloglanis EIGENMANN 1919.

Villarius RUTTER 1896.

\section{FAM. DES AMBLYCIPITIDAE}

Acrochordonichthys BLEEKER 1858. Akysis BLEEKER 1860.

Amblyceps BLYTH 1858.

Parakysis Herre 1940.

Sosia Vaillant 1902.

\section{FAMILLE DES SISORIDAE}

Aglyptosternon BLEEKER 1862.

Bagarius BLEEKER 1853.

Batasio BLYTH 1860.

Breitensteinia STEINDACHNER 1881. Callomystax GüNTHER 1864 : syn. de Batasio B. 1860.

Chiloglanis Peters 1868.

Coraglanis Hora et SILAS 1952.

Erethistes MüLLER et Troschel 1849.

Euchiloglanis REGAN 1907.

Euclyptosternum GüNTHER 1864 : syn. d'Aglyptosternon B. 1862.

Exostoma BLYTH 1860.

Gagata BleEKER 1858: syn. de Batasio B. 1860.

Glaridoglanis NORMAN 1925.

Glyptosternon Mc Clelland 1842.

Glyptosternum: vide Glyptosternon MC. 1842.

Glyptothorax BLYTH 1860: syn. de Glyptosternon MC. 1842.

Hara BLYTH 1860: syn. d'Erethistes M. et T. 1849.

Myersglanis HORA et SILAS 1952.

Nangra DAY 1876.

Oreoglanis Sмттн 1933.
Parapseudecheneis HORA et CHABANAUD 1930.

Pareuchiloglanis Pellegrin 1936.

Parexostoma Regan 1905.

Paroreoglanis Pellegrin 1936.

Propseudecheneis HORA 1937.

Pseudecheneis BLYTH 1860.

Pteroglanis Fowler 1934: pré-employé, syn. de Pteropsoglanis F. 1934.

Pteropsoglanis FOWLER 1934.

Sisor Hamilton-Buchanan 1822.

\section{FAMILLE DES AMPHILIIDAE}

Amphilius GüNTher 1864.

Andersonia BOULENGER 1900.

Anoplopterus PFEFFER 1889: syn. d'Amphilius G. 1864.

Belonoglanis BOULENGER 1902.

Chimarrhoglanis VAILlant 1897 : syn. d'Amphilius G. 1864.

Chimarrichthys SAUVAGE 1874.

Doumea SaUvage 1879.

Paramphilius Pellegrin 1907.

Paraphractura BoUlenger 1902.

Peltura Perugia 1892: pré-employé, syn. de Phractura B. 1900.

Phractura Boulenger 1900.

Trachyglanis BOULENGER 1902.

FAM. DES LEPIDOGLANIDAE

Lepidoglanis VAILLANT 1889.

FAMILLE DES CHACIDAE Chaca GraY 1831.

\section{FAMILLE DES SCHILBEIDAE}

Acanthonotus Gray 1831: pré-employé, syn. de Ailia G. 1831.

Ailia Gray 1831.

Ailichthys DAY 1871. 
Anopleutropius VAILlant 1893.

Ansorgia BOULENGER 1912: pré-employé, syn. de Ansorgiichthys W. 1935.

Ansorgiichthys WhitLey 1935: syn. d'Eutropiellus N. et LM. 1933.

Clupisoma SwaINSON 1839.

Eutropiellus Nichols et LAMONTE 1933.

Eutropiichthys BLEEKER 1862.

Eutropius MüLLER et Troschel 1849.

Gymnallabes GüNTHER 1867.

Helicophagus BLEEKER 1858.

Irvineia Trewavas 1943.

Laides JORDAN 1919.

Lais BLEEKER 1860 : pré-employé, syn. de Laides J. 1919.

Neotropis KULKARNI 1952.

Pachypterus SwaInson 1839, pré-ememployé, syn. de Phagorus MC B. 1862 .

Parailia Boulenger 1899.

Pareutropius REgAN 1920.

Physailia Boulenger 1901.

Platytropius HORA 1937.

Proeutropichthys HORA.

Proeutropius FOWLER 1936: s./g. d'Eutropius M. et T. 1849.

Pseudeutropius BLEEKER 1862.

Pseudolais Vaillant 1902.

Pusichthys SwaInson 1839: syn. de Schilbe (C.) O. 1817.

Schilbe (CUVIER) OKEN 1817.

Schilbeichthys BLEEKER 1858: syn. de Clupisoma S. 1839.

Silonia SwaINSON 1839.

Silonopangasius HORA.

Silundia Cuvier et VALENCIENNES 1840: syn. de Silonia S. 1839.

Siluranodon BLEEKER 1858.

\section{FAMILLE DES CLARIIDAE}

Allabenchelys Boulenger 1902: syn. de Clarias (G.) S. 1777.

Bathyclarias JACKSON 1959.

Channalabes GüNTHER 1873.
Clariallabes BOULENGER 1898.

Clarias Gronow 1763: non valide.

Clarias (G.) Scopoli 1777.

Clarioides DAvID 1935: s./g. de Clarias (G.) S. 1777.

Clarisilurus FOWLER 1938: syn. d'Heteropneustes M. 1839.

Cossyphus Mc Clelland 1844 : préemployé, syn. de Pagorus MC. 1844.

Dinotopteroides FOWLER 1930.

Dinotopterus BOULENGER 1906.

Dolichallabes POLL 1942.

Encheloclarias HERRE et MYERS 1937.

Heterobranchoides DAVID 1935: syn. de Clarias (G.) S. 1777.

Heterobranchus GEOFFROY-ST-HILAIRE 1818.

Heteropneustes MüLLER 1839.

Horaglanis MENON 1950.

Macropteronotus DE LACÉPÈDE 1803 : syn. de Clarias (G.) S. 1777.

Phagorus Mc Clelland 1844.

Prophagorus Smith 1939: syn. de Clarias (G.) S. 1777.

Saccobranchus CUVIER et VALENCIENNES 1840 : syn. d'Heteropneustes M. 1839.

Tanganikallabes POLL 1942.

Xenoclarias GREenWOOD 1958.

FAMILLE DES PANGASIIDAE

Nemasiluroides FOWLER 1937.

Neopangasius PoPTA 1904.

Pangasianodon CheVEY 1930.

Pangasius Cuvier et VAlenciennes 1840.

Pseudopangasius BLEEKER 1862.

Pteropangasius FOWILE 1937.

\section{FAMILLE DES MOCHOCIDAE}

Acanthocleithron Nichols et GRISсом 1917.

Atopochilus Sauvage 1879.

Brachysynodontis BLEEKER 1862. 
Euchilichthys BOULENGER 1900.

Hemisynodontis BLEEKER 1862.

Leiosynodontis BLEEKER 1862.

Microsynodontis BOULENGER 1903. Mochocus GüNTHER 1864: emend. pro Mochokus J. 1835.

Mochokus JOANNIS 1835.

Oxyglanis VINCIGUERRA 1898.

Slatinia WERNER 1906.

Synodontis CUVIER 1817.

FAM. DES MALAPTERURIDAE (= Torpedinidae)

(Malaptérures; Electric cat fishes) Malapterurus DE LACÉPÈDE 1803. Torpedo FORSKAL 1771 : non valide. Torpedo (F.) GILL 1890: syn. de Malapterurus L. 1803.

\section{FAMILLE DES HOLOGENID AE}

Hologenes GüNTHER 1863.

\section{FAM. DES HYPOPHTHALMIDAE}

Davalla BLEEKER 1858.

Hypophthalmus SPIX 1829.

Notophthalmus HYRTL 1859: préemployé, syn. d'Hypophthalmus S. 1829.

Pseudohypophthalmus BLEEKER 1862: syn. de Hypophthalmus S. 1829.

\section{FAMILLE DES PIMELODIDAE}

Abron Gistel 1848: syn. de Sorubim S. 1828.

Acentronichthys EIgenManN et EIGENMANN 1889.

Bagropsis LÜTKEN 1874.

Batrachoglanis GILL 1858: syn. de Pseudopimelodus B. 1863.

Bergiaria EIGENMANN et NORRIS 1901.

Bergiella EIgENMANN et NorRIS 1900: pré-employé, syn. de Bergiaria E. et N. 1901.
Brachyglanis Eigenmann 1912.

Brachyplatysoma BLEEKER 1862.

Brachyrhamdia MYERS 1927.

Breviglanis EIGENMANN 1909.

Caecorhamdella BORODIN 1927.

Caecorhamdia NORMAN 1926.

Callophysus Jordan 1919: vide Calophysus M. 1843.

Calophysus MÜLler 1843.

Cephalosilurus HASEMAN 1911.

Cetopsorhamdia EIGENMANN 1916.

Chasmocephalus EIGENMANN 1909.

Chasmocranus EIGENMANN 1912.

Cheirocerus EIgenmann 1917.

Conorhynchos BLEEKER 1858.

Conorhynchus BLEEKER 1863: vide Conorhynchos B. 1858.

Conostome DumérIL 1856: non valide, syn. de Conorhynchos B. 1858.

Duopalatinus EIgENMANN et EIGENMANN 1888.

Goeldiella EIGENMANN et NORRIS 1900.

Goslinia MYERS 1941.

Hemiplatystoma BLEEKER 1862 : syn. de Pseudoplatystoma B. 1862.

Hemisorubim BLEEKER 1862.

Heptapterus BLEEKER 1858.

Iheringichthys EIGENMANN et NORRIS 1900.

Imparales SchULTZ 1944.

Imparfinis EIGENMANN et NORRIS 1900.

Laimunema SAUVAgE 1884.

Leiarius BLEEKER 1862.

Leptoglanis EIGENMANN 1912.

Leptorhamdia EIGENMANN 1918.

Lophiosilurus STEINDACHNER 1876.

Luciopimelodus EIGENMANN et EIGENMANN 1888.

Malacobagrus BLEEKER 1862: syn. de Piratinga B. 1858.

Megalonema EIGENMANN 1910.

Microglanis EIGENMANN 1912.

Myoglanis EIGENMANN 1912. 
Nannoglanis BOULENGER 1887.

Nannorhamdia REgAN 1913.

Nemuroglanis EIGENMANN et EIGENMANN 1889.

Notoglanis GüNTher 1864.

Parabagrus BLEeKer 1862.

Parapimelodus LAMONTE 1933.

Pariolius COPE 1872.

Paulicea IHERING 1898.

Perrunichthys Schultz 1944.

Perugia Eigenmann et Norris 1900.

Phractocephalus Agassiz 1829.

Phrenatobius GöLdI 1898.

Pimelodella EIgENMANN et EIgENMANN 1888.

Pimelodina SteIndachner 1876.

Pimelodus DE LACÉPÈde 1803.

Pimelonotus Gill 1858: syn. de Rhamdia B. 1858.

Pimelotropis GILl 1859: syn. de Calophysus M. 1843.

Pinirampus BLEEKER 1858.

Piramutana BLEEKER 1858.

Pirarara SpIX 1829: syn. de Phractocephalus A. 1829.

Piratinga BLEEKER 1858.

Platynematichthys BLEEKER 1858.

Platypogon STARKS 1913.

Platysilurus HASEMAN 1911.

Platystoma Agassiz 1829: pré-employé, syn. de Sorubim S. 1829.

Platystomatichthys BLEEKER 1862.

Pseudariodes BLEEKER 1862: syn. de Pimelodus L. 1803.

Pseudocalophysus BLEEKER 1862 : syn. de Calophysus M. 1843.

Pseudopimelodus BLEEKER 1863.

Pseudoplatystoma BLEEKER 1862.

Pseudorhamdia BLEEKER 1862: syn. de Pimelodus L. 1803.

Pteroglanis PERASON 1924.

Pteronotus SwaInson 1839: pré-employé, syn. de Rhamdia B. 1858.

Rhamdella EIGENMANN et EIGENMANN 1888.

Rhamdia BLEEKER 1858.

Rhamdioglanis 'IHERING 1907: syn. d'Imparfinis E. et N. 1900.
Rhamdiopsis HASEMAN 1911.

Sciadeichthys BLEEKER 1858.

Sciadeoides EIGENMANN et EIGENMANN 1888.

Sciades Müller et Troschel 1849.

Sorubim SPIX 1829.

Sorubimichthys BLEEKER 1862.

Sovichthys SchULTZ 1944.

Steindachneria EIGENMANN et EIGENMANN 1888: pré-employé, syn. de Steindachneridion E. et E. 1919.

Steindachneridion EIGENMANN et EIGENMANN 1919.

Taenionema EIGENMANN et BEAN 1907 : pré-employé, syn. de Goslinia M. 1941.

Typhlobagrus RIBEIRo 1907.

Typhlobagrus RIBEIRO 1918.

Zungaro BLEEKER 1858.

Zungaropsis STEINDACHNER 1908.

FAM. DES BUNOCEPHALIDAE

Amaralia FowLer 1954.

Bunocephalichthys BLEEKER 1858.

Bunocephalus KNER 1855.

Dupouyichthys SchULTZ 1944.

Dysichthys COPE 1874.

Erustichthys FERNANDEZ-YEPEZ 1953.

Hoplomyzon MYERS 1942.

Petacara BöHLKE 1959.

Platystacus BLOCH 1794.

Platystomatos: syn. de Platystacus.

Pterobunocephalus FOWLER 1943:

s./g. de Bunocephalus K. 1855.

Xiliphius EIGENMANN 1912.

FAMILLE DES ASPREDINIDAE Agmus Eigenmann 1912.

Aspredinichthus BLEEKER 1858.

Aspredinichthys: vide Aspredinichthus B. 1858.

Aspredo Gronow 1763: non valide. Aspredo (G.) Scopoli 1777.

Chamaigenes EIGENMANN 1912.

Cotylephorus SwaINSON 1839: syn. d'Aspredo (G.) S. 1777. 
Platistus RAfINESQUe 1815.

Platystacus KLeIN 1779: non valide. Platystacus (K.) BLOCH 1794: syn. d'Aspredo (G.) S. 1777.

\section{FAMILLE DES PYGIDIIDAE}

$$
\text { (= Trichomycteridae) }
$$

Acanthopoma LüTKEN 1891.

Apomatoceros Eigenmann 1922.

Astemomycterus GUICHENOT 1860. Bathophilus MiLEs : pré-employé, syn. de Bathypygidium W. 1947.

Bathypygidium WhITLEY 1947.

Branchioica EIGENMANN 1918: syn. de Paravandellia R. 1912.

Cobitiglanis FOWLER 1914.

Eremophilus voN HuMBolt 1806.

Glanapteryx MYERS 1927.

Gyrinurus RibeIRo 1912: syn. d'Homodiaetus E. et W. 1907.

Haemomaster MYERS 1927.

Haemonaster: vide Haemomaster M. 1927.

Hatcheria EIGENMANN 1909: syn. de Eremophilus H. 1806.

Henonemus EIGENMANN et WARD 1907: syn. de Stegophilus R. 1858 ,

Homodiaetus EIGENMANN et WARD 1907.

Miuroglanis EIGENMANN et EIGENMANN 1889.

Nematogenys GIRARD 1854.

Ochmacanthus EIGENMANN 1912: syn. de Homodiaetus E. et W. 1907.

Parabranchioica DEVINCENZI 1939: syn. de Paravandellia R. 1912.

Paracanthopoma GILTAY 1935.

Parastegophilus MIRANDA-RIBEIRO 1946.

Paravandellia RIBEIRO 1912.

Pareiodon KNER 1855.

Pariodon: vide Pareiodon K. 1855.

Pariolius COPE 1872.

Plectrochilus MIRANDA-R I B EIRO 1917.

Pleurophysus RIBEIRO 1918.
Pseudostegophilus EIGENMANN et EIGENMANN 1889.

Pygidianops MYers 1944.

Pygidium MEYEN 1835.

Rhizosomichthys MILES 1943.

Scleronema EIGENMANN 1918.

Stegophilus REINHARDT 1858.

Trachypoma GIEBEL 1870: pré-employé, syn. d'Eremophilus $\mathbf{H}$. 1806.

Trichomycterus VALENCIENNES 1846 : emend. pro Trycomycterus $\mathrm{H}$. et V. 1811.

Tridens Eigenmann et EigenmanN 1889.

Tridensimilis Schultz 1944.

Tridentopsis MYERS 1925.

Trycomycterus vON HUMBOLT et VALENCIENNES 1811: syn. d'Eremophilus H. 1806.

Typhlobelus MYERS 1944.

Urinophilus EIgENMANN 1918.

Vandellia CuVier et VAlenciennes 1846.

\section{FAMILLE DES CETOPSIDAE}

Cetopsis Agassiz 1829.

Cetopsogiton EIGENMANN et BEAN 1910.

Hemicetopsis BLEEKER 1862.

Paracetopsis (GUICHENOT) BLEEKER 1862.

Paracetopsis EIGENMANN et BEAN 1907 : pré-employé, syn. de $\boldsymbol{C e}$ -

topsogiton E. et B. 1910.

Pseudocetopsis BLEEKER 1862.

\section{FAMILLE DES ARGIDAE}

Arges Cuvier et Valenciennes 1840. Astroblepus von Humbolt 1806.

Brontes CuVIER et VALENCIENNES 1840 : pré-employé, syn. de Strephon G. 1848.

Cyclopium SwaInSON 1839.

Strephon GISTEL 1848.

Stygogenes GüNTHER 1864: syn. de Cyclopium S. 1839. 


\section{FAM. DES CALLICHTHYIDAE}

Aspidoras IHERING 1907.

Brochis COPE 1872.

Callichthys Gronow 1763: non valide.

Callichthys (G.) Scopoli 1777.

Cascardura Ellis 1913: syn. d'Hoplosternum G. 1858.

Cataphractops FOWLER 1915.

Cataphractus BLoch 1793: syn. de Callichthys (G.) S. 1777.

Chaenothorax COPE 1878.

Cordorinus RAFINESQUE 1815: syn. de Corydoras L. 1803.

Corydoras DE LACÉPÈdE 1803.

Decapodon EigenManN et EIGENMANN 1888.

Ellisichthys.

Hoplisoma SwaInson 1839: syn. de Corydoras L. 1803.

Hoplosternum GILL 1858.

Osteogaster COPE 1894.

Scleromystax GüNTHER 1864.

\section{FAMILLE DES LORICARIIDAE}

Acanthicus SPIX 1829.

Acanthodemus (de Castelnau 1855) Marshall 1873.

Acanthodermus: errat. pro Acanthodemus (C.) M. 1873.

Acestra KNER 1854: pré-employé, syn. de Farlowella E. et E. 1889.

Acestridium Haseman 1911.

Ancistrus KNER 1854.

Aristommata Holmberg 1893: syn. d'Hypoptopoma G. 1868.

Canthopomus EIGENMANN 1909.

Canthopomus Eigenmann et Allen 1942.

Carinotus Lamonte 1933: s./g. de Plecostomus (G.) B. 1862.

Cheirodus EIGENMANN 1922.

Cheirododus EIGENMANN 1921.

Cochliodon HECKEL 1864.

Corymbophanes EIgenManN 1909.
Delturus Eigenmann et EigenmanN 1889.

Diapaletoplites FOWLER 1915.

Farlowella EIGENMANN et EIGENMANN 1889.

Fusiloricaria FOWLER 1940: s./g. de Loricaria L. 1758.

Harttia Steindachner 1876.

Hassar EigenManN et EigenManN 1888.

Hemiancistrus BLEEKER 1862.

Hemiloricaria BLEEKER 1862.

Hemiodon KNER 1854: pré-employé, syn. de Reganella E. 1905.

Hemiodontichthys BLEEKER 1862.

Hemipsilichthys EIGENMANN et EIGENMANN 1889.

Hisonotus Eigenmann et EIgenManN 1889.

Hypocolpterus FOWLER 1944.

Hypoptopoma GÜNTHER 1868.

Hypostomus DE LACÉPÈDE 1803 : préemployé, syn. de Plecostomus (G.) B. 1862 .

Isonotus: vide Hisonotus E. et E. 1889.

Kronichthys RIBEIRO 1908: syn. d'Hemipsilichthys E. et E. 1889.

Lamontichthys MIRANDA - RIBEIRO 1940.

Lasiancistrus REGAN 1904.

Leptoancistrus MEEK et HILDEBRAND 1916.

Lipopterichthys NORMAN 1935.

Liposarcus GÜNTHER 1864.

Lithogenes EIGENMANN 1909.

Lithoxus EIGENMANN 1912.

Loricaria LINNÉ 1758.

Loricariichthys BLEEKER 1862.

Microlepidogaster EIGENMANN et EIGENMANN 1889.

Monistiancistrus FOWLER 1940.

Neoplecostomus EIGENMANN et EIGENMANN 1888.

Otocinclus COPE 1872.

Otothyris MYERS 1927. 
Oxyloricaria BLEEKER 1862.

Oxyropsis EIgENMANN et EIgenManN 1889.

Panaque Eigenmann et EIgenmanN 1889.

Parahemiodon BLEEKER 1862: syn. de Loricariichthys B. 1862.

Pareiorhaphis RIBEIRO 1918.

Pareiorhina GosLine 1951.

Parotocinclus EIgENMANN et EIGENMANN 1889.

Plecostomus Gronow 1763 : nor. valide.

Plecostomus (G.) BLEEKER 1862.

Pogonopoma Regan 1907.

Pogonopomoides GosLINE 1951.

Pristiancistrus FOWLER 1945.

Pseudacanthicus BLEEKER 1862.

Pseudancistrus BLEEKER 1862.

Pseudohemiodon BLEEKER 1862.

Pseudoloricaria BLEEKER 1862.

Pseudorinelepis BLEEKER 1862.

Pseudosynodontis BLEEKER 1862.

Pseudotocinclus Nichols 1919.

Pterygoplichthys GILL 1858.

Reganella EIGENMANN 1905.

Rhinelepis SPIX 1829.

Rhinoglanis GüNTHER 1864.

Rineloricaria BLEEKER 1862: s./g. de Loricaria L. 1758.

Spatuloricaria SchULTZ 1944.

Stoniella FOWLER 1914.

Sturisoma SWaINSON 1839.

Thysanocara REGAN 1906.

Upsilodus RIBEIRO 1925.

Xenocara REgan 1904.

Xenomystus LüTKEN 1873: pré-employé, syn. d'Hemipsilichthys E. et E. 1889.

\section{Ordre des ANGUILLIFORMES}

Anguilliformes incertae sedis.

Dameus RAFINESQUe 1815 : non valide.
Eleuthurus RAFINESQUE 1815: non valide.

Rincoxis RAFINESQUE 1815 : non valide.

Zebriscium RAFINESQUE 1815: non valide.

Sous-ordre des ANGUILLOIDEI

FAMILLE DES DERICHTHYIDAE

Benthenchelys FOWLER 1934.

Derichthys GILl 1884.

FAMILLE DES ANGUILLIDAE (Anguilles; True Eels)

Anguilla SHAW 1803.

Tribranchus (Peters) MüLler 1844 : syn. d'Anguilla S. 1803.

FAM. DES SIMENCHELYIDAE

Conchognathus ColletT 1889: syn. de Simenchelys (G.) G. et B. 1879.

Gymnosimenchelys TANAKA 1908.

Simenchelys (GILL) GoODE et BEAN 1879.

FAM. DES XENOCONGRIDAE Xenoconger REgAN 1912.

FAM. DES MYROCONGRIDAE Myroconger GÜNTHER 1870.

FAMILLE DES MURAENIDAE (Murènes; Morays)

Aemasia Jordan et SNYDer 1901. Ahynnodontophis FOWLER 1912. Anarchias Jordan et Seale 1906. Arndha Deraniyagala 1931. Channomuraena RICHARDSON 1844. Chasmenchelys FOWLER 1944. Echidna FORSTER 1777 : pré-employé, syn. de Megaderus R. 1815. 
Enchelycore KAUP 1856.

Enchelycotte (KAUP) DUMÉRIL 1856 : non valide, syn. d'Enchelycore K. 1851 .

Enchelynassa KAUP 1855.

Eurymyctera KAUP 1856: syn. de Gymnothorax B. 1795.

Evenchelys JORDAN et EVERMANN 1902.

Fimbrinares WHITLEY 1950.

Gymnomuraena DE LACÉPÈDE 1803. Gymnopsis RAFINESQUE 1815: syn. de Gymnomuraena L. 1803.

Gymnothorax BLoch 1795.

Gymnothorax GüNTHER : préemployé, syn. de Gymnothorax B. 1795 .

Haliophis RüPPELL 1828.

Ichthyapus BRISOUT DE BARNEVILLE 1847.

Ichthyophis Lesson 1828: syn. de Gymnomuraena L. 1803.

Leihala JORDAN 1925.

Limamuraena KaUP 1856: syn. de Muraena L. 1758.

Lycodontis Mc Clelland 1844 : syn. de Gymnothorax B. 1795.

Megaderus RAFINESQUE 1815.

Molarii RICHARDSON 1844: non valide, syn. de Megaderus R. 1815 .

Muraena LINNÉ 1758.

Muraenoblenna KAUP 1856.

Muraenophis CUVIER 1798.

Muraenophis DE LACÉPÈDE 1803 : syn. de Muraena L. 1758.

Neomuraena GIRARD 1859: syn. de Muraena L. 1758.

Notorabula WHITLEY 1934.

Poecilophis Kaup 1856: syn. de Megaderus R. 1815.

Polyuranodon KaUP 1856: syn. de Gymnothorax B. 1795.

Priodonophis KaUP 1860.

Pseudechidna BLEEKER 1863.

Pseudomuraena JoHNSON 1862: syn. de Priodonophis K. 1860.

Pythonichthys POEY 1867.
Rabula JoRDAN et DAvis 1892.

Rhabdura OGILBY 1907 : syn. d'Evenchelys J. et E. 1902.

Rhinechidna BARBOUR 1908.

Rhinomuraena GARMAN 1888.

Scutia Jordan et EVERMANN 1896: syn. de Gymnomuraena L. 1803.

Scuticaria JORDAN et SNYDER 1901.

Serranguilla WhItLEY et PHILlIPS 1939.

Sidera KaUP 1856: syn. de Gymnothorax B. 1795.

Siderea Kaup 1856: syn. de Gymnothorax B. 1795.

Strophidion MC CLELLAND 1844 : syn. de Gymnothorax B. 1795.

Taeniophis KaUP 1860: syn. de Gymnothorax B. 1795.

Thaerodontis MC Clelland 1844: syn. de Gymnothorax B. 1795.

Thyrsoidea Kaup 1856: syn. de Gymnothorax B. 1795.

Uropterygius RüPPELL 1835: syn. de Gymnomuraena L. 1803.

Verdithorax WhITLEY 1931.

FAM. DES HETERENCHELYIDAE

Heterenchelys REGAN 1912.

Lophenchelys BEN-TUVIA 1953.

Panturichthys PeLlegrin 1913.

FAMILLE DES MORINGUIDAE

Anguillichthys (MOWBRAY) BREDER 1927: syn. de Stilbiscus J. et B. 1888 .

Aphthalmichthys KAUP 1856.

Cryptophthalmus FRANZ 1910: préemployé, syn. d'Unagius J. 1919.

Gordiichthys JoRDAN et DAVIS 1892.

Moringua GRAY 1831.

Pachyurus SWAINSON 1839: pré-employé, syn. de Pseudomoringua B. 1865 .

Pseudomoringua BLEEKER 1865.

Pterurus SwaInson 1839: syn. de Moringua G. 1831. 
Ptyobranchus Mc Clelland 1844 : syn. de Moringua G. 1831.

Raitaboura JORDAN 1919: vide Rataboura G. 1831.

Raitoboura JORDAN 1919 : vide Rataboura G. 1831.

Rataboura Gray 1831: syn. de Moringua G. 1831.

Stilbiscus Jordan et Bollman 1888. Unagius JORDAN 1919.

\section{Sous-ordre des CONGROIDEI}

FAM. DES NEENCHELYIDAE

Neenchelys BAMBER 1915.

\section{FAMILLE DES \\ SYNAPHOBRANCHIDAE}

Diastobranchus BARNARD 1923.

Histiobranchus GILL 1883: s./g. de Synaphobranchus J. 1862.

Histriobranchus: errat. pro Histiobranchus J. 1883.

Nettophichthys HoLt 1891.

Synaphobranchus JoHNSON 1862.

\section{FAMILLE DES ILYOPHIIDAE}

Ilyophis GILBERT 1891.

\section{FAMILLE DES DYSOMMIDAE}

Dysomma Alcock 1889.

Dysommopsis Alcock 1891.

Meadia BöHLKE 1951.

FAMILLE DES DYSOMINIDAE

Dysommina GINSBURG 1951.

FAM. DES NESSORHAMPHIDAE Nessorhamphus ScHMIDT 1930.
FAMILLE DES

MACROCEPHENCHELYIDAE

Macrocephenchelys FOWLER 1934.

\section{FAM. DES MURAENESOCIDAE}

Brachyconger BLEEKER 1865: syn. de Cynoponticus C. 1846.

Cheiromuraenesox FOWLER 1944.

Congresox GILL 1890.

Cynoponticus Costa 1846.

Muraenesox Mc Clelland 1844.

Muraenosaurus OsORIO 1909: préemployé, syn. d'Osorina W. 1953.

Osorina Whitley 1953.

Paraxenomystax REID 1940.

Phyllogramma Pellegrin 1934.

Pseudoxenomystax BREDER 1927.

FAMILLE DES

SAUROMURAENESOCIDAE

Sauromuraenesox AlcocK 1889.

FAM. DES NETTASTOMATIDAE

(= Nettastomidae) (Duck-bill eels)

Chlopsis RAFINESQUE 1810.

Dietrichthys WHITLEY 1935: s./g. de Chlopsis R. 1810.

Facciolella WHITLE 1938

Garmanichthys SeAle 1917: syn. de Chlopsis R. 1810.

Hyoprorus KöLlIKeR 1853: larve de Netiastoma R. 1810.

Nettastoma RAFINESQUE 1810.

Nettastomella FAcciola 1914.

Nettastomops STEINDACHNER 1906.

Saurenchelys PETERS 1864: syn. de Chlopsis R. 1810.

Venefica JoRDAN et DAVIS 1892.

\section{FAMILLE DES CONGRIDAE}

(Congres ; Conger eels)

Alloconger JoRdan et HuBBs 1925. Anago Jordan et HubBS 1925. 
Ariosoma Swainson 1838.

Astroconger JoRdan et HuBBs 1925. Atopichthys Garman 1899: larves de Congridae sp.

Bassanago WhItLeY 1950.

Bathycongrus OGILBY 1898.

Bathyuroconger FOWLER 1934 : s./g. d'Uroconger K. 1856.

Chiloconger MYERS et WADE 1941.

Coloconger ALCOCK 1889.

Conger HoutTuYN 1764 : vide Conger (C.) O. 1817.

Conger KLEIN 1775 : non valide.

Conger (CUVIER) OKEN 1817.

Congermuraena KAUP 1858: syn. d'Ariosoma S. 1838.

Congerodon (KAUP) BLEEKER 1856: syn. de Uroconger K. 1856.

Congrellus OGILBY 1898: syn. d'Ariosoma S. 1838.

Congrhynchus FowLER 1934.

Congrina Jordan et HuBbs 1925.

Congriscus Jordan et HuBBs 1925.

Congromuraena : vide Congermuraena $\mathrm{K} .1858$.

Congrosoma GARMAN 1899.

Congrus RichaRdSON 1844: syn. de Conger (C.) O. 1817.

Diaphanichthys PeTERS 1864: larve d'un Congridae sp.

Euleptocephalus Strömman 1895.

Forskalichthys WHITLEY 1935.

Gnathophis KAUP 1860: syn. d'Ariosoma S. 1838.

Gorgasia Meek et Hildebrand 1924.

Grammatocephalus NORMAN 1930.

Helmichthys Costa 1854: larve de Conger (C.) O. 1817.

Hildebrandia JORDAN et EVERMANN 1927.

Hoplunnis KAUP 1860.

Isognatha (DEKAY) GILL 1861: syn. de Conger (C.) O. 1817.

Japonoconger AsANO 1958.

Leptocephalichthys BLEEKER 1856: forme larvaire d'un Congridae sp.

Leptocephalus GroNow 1763: non valide.
Leptocephalus ScOPOLI 1777.

Mayerina SILVESTER 1915.

Metopomycter GILBERT 1905.

Microcephalocongrus FOWLER

1934: s./g. de Bathycongrus O. 1898.

Microconger FOWLER 1912.

Neoconger GIRARD 1859.

Nesocongrus WhITLEY 1935.

Nettodarus WhITLEY 1952.

Nystactes BöHLKE 1957: pré-employé, syn. de Nystactichthys B. 1958 .

Nystactichthys BöHLKE 1958.

Ophisoma SwaINSON 1839: syn. d'Ariosoma S. 1838.

Oxyconger BLEEKER 1865.

Oxyurus RAFINESQUE 1810: larve d'un Congridae sp.

Poeciloconger GüNTHER 1871.

Poutawa GrIfFIN 1936.

Promyllantor Alcock 1890.

Pseudophichthys Roule 1915.

Rhechias JORDAN 1921.

Rhynchoconger JORDAN et HuBBS 1925.

Rhynchocymba JORDAN et HUBBS 1925.

Scalanago WHITLEY 1935.

Silvesterina FOWLER 1934.

Thyreoconger W A DE 1946: syn. d'Ariosoma S. 1838.

Tilurus KöLLIKER 1853: larve d'un Congridae sp.

Todarus Grassi et CALANDRUCcio 1896 : pré-employé, syn. de Nettodarus W. 1952.

Uranoconger FOWLER 1934.

Uroconger KaUP 1858.

Veternio SNYDER 1904.

Xarifania KLAUSEWITZ et EIBL-EIBESFELDT 1959.

Xenomystax GILBert 1891.

FAM. DES HETEROCONGRIDAE

Heteroconger BLEEKER 1868.

Taenioconger HERRE 1923. 
FAMILLE DES ECHELIDAE

$(=$ Myridae $=$ Chilorhinidae $)$

Ahlia Jordan et Davis 1892.

Arenichthys Beebe et TeE-Van 1938: syn. de Garmanichthys SEALE 1917.

Bathymyrus Alcock 1890.

Brachyconger NORMAN 1922: syn. de Chilorhinus L. 1851.

Brachyconger REGAN 1922: syn. de Chilorhinus L. 1851.

Chilorhinus LüTKEN 1851.

Chrinorhinus RIVERO 1932.

Echelus RAFINESQUE 1810.

Endeconger JORDAN 1923: syn. de Chilorhinus L. 1851.

Eomyrophis WHITLEY 1950.

Eomyrus EASTMAN 1905: syn. d'Eomyrophis W. 1958.

Garmanichthys SEALE 1917: vide Nettastomatidae.

Hesperomyrus MYers et STOREY 1939.

Holopterura COPE 1871.

Kaupichthys SCHULTZ 1943.

Leptenchelys MYERS et WADE 1941.

Merinthichthys RIVERO 1934.

Muraenichthys BLEEKER 1853.

Myrophis LüTKEN 1851.

Myropterura OGILBY 1897.

Myrus CUVIER 1817: syn. d'Echelus R. 1810 .

Myrus KaUP 1856: syn. d'Echelus R. 1810 .

Parabathymyrus KamoHaRA 1938.

Paramyrus GüNTHeR 1870.

Pseudomyrophis WADE 1946.

Scolenchelys OgILBY 1897: syn. de Muraenichthys B. 1853.

Sinomyrus LIN 1933.

Verma JoRdan et EVERMANN 1896.

FAMILLE DES OPHICHTHYIDAE

(Serpents de mer; Snake-eels)

Acanthenchelys REgAN 1922.

Achirophichthys BLEEKER 1865: larve de Brachysomophis K. 1856. Anepistomon G I T TEL 1848: syn. d'Ophisurus L. 1800.
Anguisurus KaUP 1856: syn. de Caecula V. 1794.

Aplatophis BöHLKE 1956.

Apterichthe DUMÉRIL 1856: non valide, syn. de Sphagebranchus B. 1795 .

Apterichthys DUMÉRIL 1806: syn. de Sphagebranchus B. 1795.

Bascanichthys JORDAN et DAVIS 1892.

Brachycheirophis FOWLER 1944.

Brachysomophis KAUP 1856.

Branderius RAFINESQUE 1815: syn. de Sphagebranchus B. 1795.

Caecilia DE LACÉPÈDE 1800 : pré-employé, syn. de Sphagebranchus B. 1795 .

Caecula VAHL 1794.

Calamuroena WhItLeY 1944.

Callechelys KAUP 1856.

Caralophia BÖHLKE 1955.

Centrurophis K A U P 1856: syn d'Ophichthus A. 1787.

Chlevastes JORDAN et SNYDER 1901.

Cirrhimuraena KAUP 1856.

Cirricaecula SCHULZE 1953.

Coecilophis K AUP 1856: syn. d'Ophichthus A. 1787.

Cogrus Rafinesque 1810.

Colubrina DE LACÉPÈDE 1803: validité douteuse.

Crotalophis: errat. pro Crotalopsis K. 1860 .

Crotalopsis Kaup 1860.

Cryptopterenchelys FowLER 1925: syn. d'Ophichthus A. 1787

Cryptopterus KAUP 1860: pré-employé, syn. d'Ophichthus A. 1787.

Cryptopterygium GINSBURG 1951.

Cyclophichthys WHITLEY 1952:

s./g. de Malvoliophis W. 1934.

Dalophis RAFINESQUe 1810: syn. de Caecula V. 1794.

Echiopsis KaUP 1856: syn. de Mys triophis K. 1856.

Elapsopsis KaUP 1856: syn.

d'Ophichthus A. 1787. 
Giscenchelys FOWLER 1944: s./g. d'Ophichthus A. 1787.

Helmictis RAFINESQUE 1810: syn. de Caecula V. 1794.

Hemerorhinus WEBER et BEAUFORT 1905.

Herpetoichthys KAUP 1856: syn. d'Ophichthus A. 1787.

Ichthyapus BRISOUT DE BARNEVILLE 1847: syn. de Sphagebranchus B. 1795.

Jenkinsiella JORDAN et EVERMAN 1905.

Lamnostoma BLEEKER 1853.

Leiuranus BLEEKER 1853.

Leiurus KAUP 1856: pré-employé, syn. de Chlevastes J. et S. 1901.

Leiurus (K.) DUMÉRIL 1856 : pré-employé, syn. de Chlevastes J. et S. 1901 .

Leptognathus SwaINSON 1839: syn. d'Ophisurus L. 1800.

Leptorhinophis KaUP 1856: syn. de Scytalophis K. 1856.

Leptorhynchus SMITH 1847 : pré-employé, syn. d'Ophisurus L. 1800.

Letharchus GoOdE et BEAN 1882.

Machaerenchelys FOWLER 1938.

Macrodonophis POEY 1867: syn. de Crotalopsis K. 1860.

Malvoliophis WHITLEY 1934.

Microdonophis KAUP 1856: syn. d'Ophichthus A. 1787.

Muraenopsis: errat. pro Muraenophis K. 1856.

Muraenophis KAUP 1856.

Myrichthys GIRARD 1859.

Myrichthys GoSLINE 1951.

Mystriophis KaUP 1856.

Omochelys FOWLER 1918.

Ophichthus AHL 1787.

Ophichthys: vide Ophichthus A. 1787.

Ophis Turton 1807 : syn. d'Ophichthus A. 1787.

Ophisternon MC CLELLAND 1844.

Ophisurapus Kaup 1856: syn. de Sphagebranchus B. 1795.

Ophisurus DE LACÉPÈDE 1800.
Ophithorax MC CIELI AND 1844 : syn. d'Ophichthus A. 1787.

Oxystomus RAFINESQUE 1810: syn. d'Ophichthus A. 1787.

Phaenomonas MYERS et WADE 1941.

Phyllophichthus GosLINE 1951.

Pisodonophis KAUP 1856.

Pisodontophis: errat. pro Pisodonophis K. 1856.

Pisoodonophis: syn. de Pisodonophis K. 1856.

Poecilocephalus KAUP 1856: syn. d'Ophichthus A. 1787.

Pogonophis MYERS et WADE 1941.

Pterurus RAfINESQUe 1810: syn. de Caecula V. 1794.

Ptyobranchus Mc Clelland 1844.

Quassinemus: vide Quassiremus J. et D. 1892.

Quassiremus JoRDAN et DAVIS 1892.

Schultzidia GosLINE 1951: s./g. de Muraenichthys (Echelidae).

Scytalichthys JORDAN et DAVIS 1892.

Scytallurus (KAUP) DuMÉRIL 1856: syn. de Caecula V. 1794.

Scytalophis KAUP 1856.

Sphagebranchus BLOCH 1795 : s./g. de Caecula V. 1794.

Stethopterus BLEEKER 1853 : syn. de Leiuranus B. 1853.

Syletophis WHITLEY 1950: syn. d'Omochelys F. 1918.

Syletor JORDAN 1918: syn. d'Omochelys F. 1918.

Typhlotes Fischer 1813: syn. de Sphagebranchus B. 1795.

Uranichthys POEY 1867: syn. d'Ophichthus A. 1787.

Xyrias JORDAN et SNYDER 1901.

Yirkkala WhITLEY 1940.

Zonophichthus WHITLEY 1930.

FAM. DES AVOCETTINOPSIDAE

Avocettinops ROULE et BERTIN 1924. 


\section{Sous-ordre des NEMICHTHYOIDEI}

FAM. DES NEMICHTHYIDAE (Thread-eels)

Avocettina Jordan et Davis 1892: pré-employé, syn. de Borodinula W. 1931.

Belonopsis BRANDT 1854: syn. de Nemichthys R. 1848.

Borodinula WHITLEY 1931.

Ceromitus WeBer 1913.

Gavialiceps Alcock 1889.

Gavialichthys.

Labichthys GILL et RYDER 1883.

Leptorhynchus Lowe 1852: pré-employé, syn. de Nemichthys R. 1848.

Nematoprora GILBERT 1905.

Nemichthys RICHARDSON 1848.

Platuronides Roule et BERTIN 1924. Spinivomer GILL et RYDER 1883.

Stemonidium GILBERT 1905.

FAMILLE DES CYEMIDAE

Cyema GüNTHer 1878.

FAM. DES SERRIVOMERIDAE

Bertinulus WhITLEY 1950.

Paraserrivomer Roule et ANGEL 1931.

Serrivorier GILL et RYDER 1883.

FAM. DES HETEROMYRIDAE

Heteromyrus PIETSCHMAN 1936.

Ordre des SYMBRANCHIFORMES

FAM. DES SYMBRANCHIDAE

Aotea Phillipps 1926.

Furmastix WhITLEY 1951.

Macrotrema REGAN 1912.
Ophisternon Mc Clelland 1844 : syn. de Symbranchus B. 1795.

Pluto HubBs 1938 : pré-employé, syn. de Furmastix W. 1951,

Symbranchus BLOCH 1795.

Synbranchus B LOCH 1795: vide Symbranchus B. 1795.

Tetrabranchus BLEEKER 1851: syn. de Symbranchus B. 1795.

Typhlobranchus BLOcH et SCHNEIDER 1801: validité douteuse.

Typhlosymbranchus PELLEGRIN 1922.

Unibranchapertura DE LACÉPÈDE 1803: syn. de Symbranchus B. 1795.

Unipertura (KAUP) DUMÉRIL 1856: syn. de Symbranchus B. 1795.

FAMILLE DES AMPHIPNOIDAE

Amphipnous Müller 1839.

Ophiphthys SwaINSON 1839: pré-employé, syn. d'Amphipnous M. 1839.

Pneumabranchus Mc Clelland 1844: syn. d'Amphipnous M. 1839.

\section{FAMILLE DES FLUTIDAE $(=$ Monopteridae $)$}

Apterigia BASILEWSKI 1855 : syn. de Fluta B. et S. 1801.

Fluta Bloch et SCHNEIDER 1801. Monopterus (DE LACÉPÈDE) DUMÉRIL 1806: pré-employé, syn. de Fluta B. et S. 1801.

Ophicardia Mc Clelland 1844 : syn. de Fluta B. et S. 1801.

\section{Ordre des ALABIFORMES}

FAMILLE DES ALABIDAE

Alabes (CuVIER) OKEN 1817.

Cheilobranchus RICHARDSON 1844 : syn. d'Alabes (C.) O. 1817. 
Ordre des NOTACANTHIFORMES

FAMILLE DES HALOSAURIDAE Aldrovandia GoODE et BEAN 1895. Halosaurichthys ALCocK 1889.

Halosauropsis COLlett 1896.

Halosaurus JoHNSON 1863.

FAMILLE DES LIPOGENYIDAE Lipogenys Goode et BEAN 1894.

FAM. DES NOTACANTHIDAE

Acanthonotus BLOCH 1797: syn. de Notacanthus B. 1787.

Campylodon FaBRICIUS 1793: syn. de Notacanthus B. 1787.

Gigliolia Goode et BeAN 1894.

Gnathacanthonotus FOWLER 1934: s./g. de Polyacanthonotus B. 1874.

Macdonaldia Goode et BeAN 1894.

Notacanthus BLOCH 1787.

Paradoxichthys GigLIOLI 1882: syn. de Polyacanthonotus B. 1874.

Polyacanthonotus BLEEKER 1874.

Teratichthys GigLIOLI 1882: pré-employé, syn. de Polyacanthonotus B. 1874.

\section{Ordre des PERCOPSIFORMES}

FAMILLE DES PERCOPSIDAE (Sand-rollers or Trout-perch)

Columbia Eigenmann 1892.

Percopsis Agassiz 1848.

Salmoperca THOMPSON 1851 : syn. de Percopsis A. 1848.

FAM. DES APHREDODERIDAE (Pirate-perch)

Aphododerus : errat. pro Aphredoderus C. et V. 1833.
Aphredoderus CUvier et VALenCIENNES 1833.

Aphrodedirus: errat. pro Aphredoderus C. et V. 1833.

Asternotremia Nelson 1877: syn. d'Aphredoderus C. et V. 1833.

Sternotremia Nelson 1876: syn. d'Aphredoderus C. et V. 1833.

Ordre des AULOSTOMIFORMES

FAMILLE DES FISTULARIIDAE

(Bouche-en-flûte ; cornet-fishes)

Aulus (COMMERson) DE LACÉPÈde 1803: non valide.

Cannorhynchus CANTOR 1849: syn. de Fistularia L. 1758.

Fistularia LINNÉ 1758.

Flagellaria GRONOW 1854.

Petimbuabo CATESBY 1771: non valide.

Solenostomus GroNow 1763 : non valide.

Solenostomus KLEIN 1778: non valide.

Solenostomus BROWNE 1789 : non valide.

Solenostomus (Gronow) Gill 1861: syn. de Fistularia L. 1758.

FAMILLE DES AULOSTOMIDAE (Trompette-de-mer ; Trumpet-fishes)

Aulostoma: vide Aulostomus L. 1803.

Aulostomus DE LACÉPÈDE 1803.

Polypterichthys BLEEKER 1853: syn. d'Aulostomus L. 1803.

FAMILLE DES CENTRISCIDAE (= Amphisiliidae) (Bécasses-de-mer) Acentrachme GILL 1862: syn. de Centriscus L. 1758.

Aeoliscus Jordan et Starks 1902. Amphisile CUVIER 1817: syn. de Centriscus L. 1758.

Amphisile (KLEIN) GILl 1862: syn. d'Aeoliscus J. et S. 1902. 
Amphisilen KLEIN 1775 : non valide. Amphisyle: errat. pro Amphisile C. 1817.

Centriscus LINNÉ 1758.

\section{FAMILLE DES \\ MACRORHAMPHOSIDAE}

Centriscops GILL 1862.

Limiculina Fowler 1907: syn. de Centriscops G. 1862.

Macrognathus GroNow 1854: syn. de Macrorhamphosus L. 1803.

Macrorhamphosus DE LACÉPÈDE 1803.

Notopogon Regan 1914.

Orthichthys GILL 1862.

Scolopacichthys REGAN 1914.

\section{AULOSTOMIFORMES INCERTAE SEDIS}

Pomatias Bloch et SchNeIDER 1801 : syn. de Triurus L. 1800.

Triurus DE LACÉPÈDE 1800 .

\section{Ordre des BELONIFORMES}

\section{Sous-ordre des BELONOIDEI}

FAMILLE DES BELONIDAE

(Aiguillettes ou Orphies, Hound-fishes or Needle-fishes)

Ablennes Jordan et Fordice 1886. Athlennes JORDAN et Fordice 1886: errat. pro Ablennes J. et F. 1886.

Belone CUVIER 1817.

Busuanga HerRe 1930.

Deltatylosurus MARTIN 1954.

Djulongius Whitley 1935.

Dorybelone FOWLER 1944.

Eurycaulus OGILBY 1908: pré-employé, syn. de Platybelone F. 1919.
Lewinichthys WHITLEY 1933.

Lhotskia WHITLEY 1933.

Mastacembelus KLEIN 1775 : non var lide.

Petalichthys Regan 1911.

Platybelone FOWLER 1919.

Potamorrhaphis GüNTHER 1866.

Pseudotylosurus FERNANDEZ-YEPEZ 1948.

Ramphistoma : errat. pro Raphistoma R. 1815.

Ramphistoma SwaINSON 1839: syn. de Belone C. 1817.

Raphiobelone FOWLER 1934.

Raphistoma RAFINESQUE 1815: syn. de Belone C. 1817.

Stenocaulus OgILby 1908.

Strongylura VAN Hasselt 1824.

Thalassosteus JORDAN, EVERMANN et TANAKA 1927.

Tropidocaulus OgILby 1919: syn. de Platybelone F. 1919.

Tylosurus Cocco 1833

Xenentodon REgAN 1911.

FAM. DES SCOMBRESOCIDAE (Balaou, Sauries)

Cololabis GILL 1896.

Grammiconotus CosTA 1862.

Sayris RAFINESQUE 1810: syn. de Scombresox L. 1803.

Scombresox DE LACÉPÈdE 1803.

Sous-ordre des EXOCOETOIDEI

FAM. DES HEMIRHAMPHIDAE (Demi-becs, Half-beaks)

Ardeapiscis WhItLey 1931.

Arrhamphus GüNTHer 1866.

Chriodorus Goode et BeAN 1882.

Dermatogenys PeTERS 1865: emend. pro Dermogenys H. 1823.

Dermogenys Van Hasselt 1823.

Eulepidorhamphus FOWLER 1919.

Euleptorhamphus GILL 1859. 
Farhians WHITLEY 1930.

Hemirhamphodon BLEEKER 1866. Hemirhamphus CUVIER 1817.

Hyporhamphus GILL 1859: s./g. d'Hemirhamphus C. 1817.

Ichthyacus FERNANDEZ-YEPEZ 1948. Labidorhamphus FOWLER 1905.

Loligorhamphus WHITLE 1931.

Melapedalion FOWLER 1934.

Nomarhamphus WEBER 1922.

Nomorhamphus: errat. pro Nomarhamphus W. 1922.

Odontorhamphus WEED 1933.

Oxyporhamphus GILL 1863.

Reporhamphus WHITLEY 1931.

Rhamphodermogenys FOWLER et BEAN 1922: s./g. de Dermogenys H. 1823.

Rhynchorhamphus FOWLER 1929: s./g. d'Hemirhamphus C. 1817.

Zenarchopterus GILL 1863.

\section{FAMILLE DES EVOLANTIIDAE}

Evolantia Heller et SNOdgrass 1903.

Hemiexocoetus Fowler 1901: syn. d'Evolantia H. et S. 1903.

\section{FAMILLE DES EXOCOETIDAE}

(Poissons-volants, Flying-fishes)

Cheilopogon Lowe 1840: syn. de Cypselurus S. 1839.

Chirodorus Mc CoY 1848.

Cypselurus Swainson 1839.

Cypsilurus: errat. pro Cypselurus S. 1839.

Danichthys BruUN 1934: syn. de Cypselurus S. 1839.

Exocoetus LINNÉ 1758.

Exonautes Jordan et EVERMANN 1896.

Fodiator JoRDAN et MEEK 1885.

Halocypselurus WEINLAND 1858 : syn. d'Exocoetus L. 1758.
Hirundinichthys BREDER 1928.

Hirundo CATESBY 1771: non valide. Maculocoetus WHITLEX et COLEFAX 1938.

Parexocoetides FOWLER 1944.

Parexocoetus BLEEKER 1866.

Prognichthys BREDER 1928.

Ptenichthys Müller 1843: syn. de Cypselurus S. 1839.

Ptenonotus OgILby 1908.

Pterichthus (COMMERSON) DE LACÉPÈDE 1803 : non valide, syn. d'Exocoetus L. 1758.

\section{Ordre des BATHYCLUPEIFORMES}

FAM. DES BATHYCLUPEIDAE Bathyclupea Alcock 1891.

\section{Ordre des GASTEROSTEIFORMES}

\section{FAM. DES GASTEROSTEIDAE (Epinoches)}

Apeltes DEKAY 1842.

Culaea WhITLEY 1950.

Eucalia JORDAN 1876: pré-employé, syn. de Culaea W. 1950.

Gasteracanthus Pallas 1811: syn. de Gasterosteus L. 1758.

Gasterostea SAuvage 1874: syn. de Pygosteus (B.) G. 1861.

Gasterosteus LINNÉ 1758.

Gastraea SAuvage 1874 : syn. de Spinachia C. 1817.

Gladiunculus JoRDAN et EVERMANN 1927: syn. de Gasterosteus L. 1758.

Leiurus Swainson 1839 : syn. de Gasterosteus L. 1758.

Polycanthus Swainson 1839: syn. de Spinachia C. 1817.

Pungitius Costa 1846.

Pygosteus BreVOoRT 1862: non valide. 
Pygosteus (B.) GILL 1861.

Spinachia CUVIER 1817.

FAM. DES AULORHYNCHIDAE Aulichthys Brevoort 1862.

Auliscops Peters 1866: syn. d'Aulorhynchus G. 1861.

Aulorhynchus GILL 1861.

FAMILLE DES INDOSTOMIDAE

Indostomus PRASHAD et MUKERJI 1929.

Ordre des AMBLYOPSIFORMES

FAMILLE DES AMBLYOPSIDAE (Cave-Blind Fishes)

Amblyopsis DeKay 1842.

Chologaster AgASsIZ 1853.

Forbesella JORDAN et EVERMANN 1927.

Troglichthys EIGENMANN 1899.

Typhlichthys GIRARD 1859.

\section{Ordre des PHALLOSTETHIFORMES}

FAM. DES PHALLOSTETHIDAE Acanthostethus Herre 1939 : pré-employé, syn. de Manacopus H. 1940.

Ceratostethus MYERS 1937.

Ctenophallus Herre 1939.

Gulaphallus HERRE 1925.

Manacopus HerRe 1940.

Neostethus REgAN 1916.

Phallostethus REGAN 1913.

Phenacostethus MYERS 1928.

Plectrostethus MYERS 1935.

Sandakanus HERRE 1942: s./g. de Neostethus R. 1916.

Solenophallus AURICH 1937.
Ordre des MUGILIFORMES

FAM. DES NANNATHERINIDAE

Nannatherina REGAN 1906.

FAMILLE DES BEDOTIIDAE

Betodia REgAN 1903.

FAM. DES MELANOTAENIIDAE

Aida de Castelnau 1875: syn. de Melanotaenia G. 1862.

Aidaprora WhItLeY 1935.

Amneris Whitley 1935.

Anisocentrus REGAN 1914.

Aristeus DE CASTELNAU 1879: préemployé, syn. de Melanotaenia G. 1862 .

Atherinosoma Castelnau 1872.

Centratherina REGAN 1914.

Chilatherina REGAN 1914.

Glossolepis WEBER 1907.

Lomanetia WHITLEY 1936.

Melanotaenia GILL 1862.

Nematocentris Peters 1870: syn. de Melanotaenia G. 1862.

Neoatherina DE CASTELNAU 1875: syn. de Melanotaenia G. 1862.

Pranesus WhItley 1864.

Pseudomugil KNER 1864.

Rhadinocentrus REGAN 1914.

Rhombatractus GILL 1894: syn. de Melanotaenia G. 1862.

Rhombosoma REGAN 1914.

Strabo KNER et STEINDACHNER 1866 : syn. de Melanotaenia G. 1862.

Telmatherina BOULENGER 1897.

Zantecla DE CASTELNAU 1873: syn. de Melanotaenia G. 1862.

FAMILLE DES SPHYRAENIDAE

(Barracuda ou Brochets-de-mer)

Acus (Plumier) De Lacépède 1803 : non valide, syn. de Sphyraena R. 1793. 
Agriosphyraena FoWLER 1903.

Australuzza Whitley 1947: syn. de Sphyraena R. 1793.

Callosphyraena Sмгтн 1956: syn. de Sphyraena R. 1793.

Indosphyraena SмIтH 1956: syn. de Sphyraena R. 1793.

Sphoerina SwaInSoN 1839: errat. pro Sphyraena R. 1793.

Sphyraena KLEIN 1778: non valide.

Sphyraena RösE 1793.

Sphyraena Bloch et SCHNEIDER 1801: syn. de Sphyraena R. 1793.

Sphyraenella SMiтH 1956: syn. de Sphyraena R. 1793.

Umbla CATESBY 1771: non valide, syn. de Sphyraena R. 1793.

- FAMILLE DES MUGILIDAE

Aeschrichthys MaCleaY 1883.

Agonostomus BenNetT 1830.

Albula CATESBy 1771: non valide.

Aldrichetta WhITLEY 1945.

Arnion Gistel 1848: syn. de Mugil L. 1758.

Cephalus (Plumier) DE LACÉPÈde 1803 : non valide, syn. de $\mathbf{M u}$ gil L. 1758.

Cestraeus Cuvier et Valenciennes 1836.

Cestreus KLEIN 1777 : non valide.

Chaenomugil GILL 1863: syn. de Chelon RösE 1793.

Chelon RösE 1793.

Crenimugil Schultz 1946.

Dajaus Cuvier et Valenciennes 1836.

Ellochelon WHITLEY 1930.

Gonostomyxus Mc Donald 1869.

Gracilimugil WHITLEY 1941.

Heteromugil Schultz 1946.

Joturus POEY 1860.

Liza Jordan et SWAIN 1884: s./g. de Mugil L. 1758.

Moolgarda WhitLey 1945.

Mugil LINNÉ 1758.

Myxus Günther 1861.

Neomugil Vaillant 1894.
Neomyxus SteINDACHNER 1878.

Nestis Cuvier et VAlenciennes 1836: syn. d'Agonostomus B. 1830 .

Oedalechilus FOWLER 1903.

Oxymugil WHITLEY 1949.

Planiliza Whitley 1945: s./g. de Moolgarda W. 1945.

Plicomugil SchUltz 1953.

Pteromugil SмIтH 1948.

Querimana JORDAN et GILBERT 1882.

Rhinomugil GILL 1863.

Sicamugil FOWLER 1939.

Squalomugil OgIlby 1908: syn. de Rhinomugil G. 1863.

Strializa SмIтн 1948.

Trachystoma OGILBy 1887.

Valamugil Sмітн 1948.

Xenomugil Schultz 1946.

Xenorhynchichthys REGAN 1908.

FAMILLE DES ATHERINIDAE (Prêtres)

Adenops Schultz 1948.

Alepidomus FOWLER 1944.

Allanetta WHITLEY 1943.

Aphia Risso 1826: juvénile d'Atherina L. 1758.

Archomenidia Jordan et HuBBS 1919.

Argyrea Dekay 1842: syn. de Menidia B. 1837.

Atherina LINNÉ 1758.

Atherinason WhITLEY 1934.

Atherinella STEINDACHNER 1875.

Atherinichthys BLEEKER 1853: syn. de Chirostoma S. 1839.

Atherinoides BLEEKER 1853: syn. de Chirostoma S. 1839.

Atherinomorus FowLer 1903: syn. d'Hepsetia B. 1837.

Atherinops STEINDACHNER 1875.

Atherinopsis GIRARD 1854.

Atherion Jordan et Starks 1901.

Atherthyrina FOWLER 1959. 
Austroatherina MARRERo.

Austromenidia HuBBs 1918.

Basilichthys GIRARD 1854.

Basilichthys Аввотт 1899: pré-employé, syn. d'Austromenidia $\mathrm{H}$. 1918.

Cauque Eigenmann 1929.

Charalia BUEN 1948: s./g. d'Eslopsarum J. et E. 1896.

Charisella FOWLER 1939.

Chirostoma SwaInson 1839.

Coleotropis MYERS et WADE 1942.

Colpichthys HuBBs 1918.

Craterocephalus Mc CULLOCH 1912.

Eslopsarum JORDAN et EVERMANN 1896.

Euryarges MYERS et WADE 1942: s./g. de Nectarges M. et W. 1942.

Eurystola JoRDAN et EVERMANN 1896.

Gastropterus COPE 1878: syn. de Basilichthys G. 1854.

Hepsetia BONAPARTE 1837: syn. d'Atherina L. 1758.

Heterognathus GIRARD 1854 : syn. de Chirostoma S. 1839.

Hubbesia JORDAN 1919

Hubbsiella BREDER 1936.

Hypoatherina SCHULTZ 1948.

Ischnomembras FOWLER 1903: syn. de Menidia B. 1837.

Iso JORDAN et STARKS 1901.

Kirtlandia JoRDAN et EVERMANN 1896: syn. de Membras B. 1837.

Kronia RIBEIRO 1915.

Labidesthes COPE 1870.

Lethostole JORDAN et EVERMANN 1896.

Leuresthes JORDAN et GILBERT 1880. Melaniris MeEK 1902: syn. d'Atherithyrina F. 1959.

Melanorhinus MetzelaAR 1920.

Membras BonAPARTE 1837.

Menidia BONAPARTE 1837.

Menidiella Schultz 1948.

Mugilops MEEK et Hildebrand 1924: syn. de Melanorhinus M. 1920.
Nectarges MYERS et WADE 1942.

Notocheirus ClARK 1937.

Ocotlanichthys BUEN 1948: s./g. de Chirostoma S. 1839.

Odontesthes Evermann et Kendall 1906.

Otalia BUEN 1948.

Palmichthys BUEN 1948: s./g. de Chirostoma S. 1839.

Paratherina AURICH 1935.

Phoxargyrea Fowler 1903 : syn. de Menidia B. 1837.

Pisciregia Аввотт 1899: syn. de Basilichthys G. 1854.

Poblana BUEN 1948.

Pranesella Whitley 1934.

Protistius COPE 1874.

Pseudothyrina RIBEIRA 1915.

Quirichthys WhITLEY 1951.

Quiris WHITLEY 1950: pré-employé, syn. de Quirichthys W. 1951.

Rheocles Jordan et HubBs 1919.

Rheocloides Nichols et LA MONTE 1931.

Stenatherina SchULTZ 1948.

Taeniomembras OGILBY 1898: syn. d'Atherina L. 1758.

Thoracatherina FOWLER 1941.

Thyrina JORDAN et CULVER 1895: pré-employé, syn. d'Atherthyrina F. 1959.

Thyrinops HuBBs 1918.

Tropidostethops Schultz 1950: syn. d'Iso J. et S. 1901.

Tropidostethus OGILBY 1895: préemployé, syn. d'Iso J. et S. 1901.

Tupa BUEN 1954: s./g. d'Odontesthes E. et K. 1906.

Xenatherina REGAN 1907.

Xenomelaniris Schultz 1948.

Yaci BUEN 1954.

Ordre des POLYNEMIFORMES

FAMILLE DES POLYNEMIDAE

Eleutherochir BLEEKER 1879. 
Eleutheronema BLEEKER 1862.

Filimanus MYERS 1936.

Galeoides GÜNTHER 1860.

Pentanemus GÜNTHER 1860.

Polistonemus GILL 1861.

Polydactylus DE LACÉPÈDE 1803: syn. de Polynemus L. 1758.

Polynemus LINNÉ 1758.

Polynemus GILl 1861 : syn. de Pentanemus G. 1860.

Trichidion KLEIN 1775: non valide. Trichidion (K.) GILL 1861 : syn. de Polynemus L. 1758.

\section{Ordre des \\ CYPRINODONTIFORMES}

\section{FAM. DES CYPRINODONTIDAE (Killi-fishes)}

Acanthocephalus EIGENMANN 1907 : syn. de Lebistes F. 1862.

Adinia GIRARD 1859.

Adiniops MYERS 1924.

Allophallus HuBBs 1936: syn. de Carlhubbsia W. 1953.

Anatolichthys Kosswig et SözER 1944.

Andreasenius Clausen 1959: s./g. de Procatopus B. 1904.

Aphaniops Hoedeman 1951.

Aphanius NaRdo 1827.

Aphyosemion MYers 1924: syn. de Aplocheilus M. C. 1839.

Aplocheilichthys BLEEKER 1861 : syn. d'Aplocheilus M. C. 1839.

Aplocheilus Mc Clelland 1839.

Arizonichthys NichOLS 1940.

Austrofundulus MYERS 1932.

Borborys (BROUSSONET) GoODE et BEAN 1885: non valide, syn. de Fundulus L. 1803.

Callopanchax MYERS 1933: s./g. d'Aplocheilus M. C. 1839.

Carlhubbsia WHITLEY 1953.

Chriopeoides FOWLER 1939.
Chriopeops FOWLER 1916.

Crenichthys HuвBs 1932.

Cualac Mille 1956.

Cubanichthys HuвBs 1926.

Cynolebias STEINDACHNER 1876.

Cynopanchax AHL 1928.

Cynopoecilus REgAN 1912: syn. de Cynolebias S. 1876.

Cyprinodon DE LACÉPÈDE 1803.

Dicerophallus Alvarez 1952.

Encrotes Gistel 1848: syn. de Cyprinodon L. 1803.

Epiplatys GILL 1863.

Floridichthys HuBвS 1926

Fontinus Jordan et EvermanN 1896.

Fundulichthys BLEEKER 1859.

Fundulopanchax MYERS 1924.

Fundulosoma AHL 1924.

Fundulus DE LACÉPÈDE 1803.

Galasaccus FOWLER 1916.

Gambusinus JoRDAN et EvermanN 1896: syn. de Xenisma J. 1877.

Garmanella HuBBs 1936.

Haplochilichthys GüNTHER syn. d'Aplocheilus M.C. 1839.

Haplochilus GüNTHER : syn. d'Aplocheilus M. C. 1839.

Horaichthys KULKARNI 1940.

Hubbsichthys Schultz 1950.

Hubbsina BUEN 1941.

Hydrargira DE LACÉPÈDE 1803 : syn. de Fundulus L. 1803.

Hydrargyra : vide Hydrargira L. 1803.

Hypsopanchax MYERS 1924.

Jenkinsia JORDAN et EVERMANN 1896.

Jordanella GoODE et BEAN 1879.

Kosswigichthys SÖZER 1942.

Lacustricola MYERS 1924: syn. d'Aplocheilichthys B. 1861.

Lamprichthys REGAN 1911: syn. de Mohanga B. 1911.

Lebia (CUVIER) OKEN 1817 : syn. de Cyprinodon L. 1803.

Lebias Cuvier 1817: syn. d'Aphanius N. 1827. 
Lebistes DE FILIPPI 1862.

Lembesseia FOWLER 1949.

Leptolebias MYERS 1957: s./g. de Cynolebias S. 1876.

Leptolucania MYERS 1924.

Lucania GIRARD 1859.

Lycocyprinus Peters 1868.

Micristius Gill 1865: syn. de Zygonectes A. 1853.

Micromugil GULIA 1861: syn. d'Aphanius N. 1827.

Micropanchax MYERS 1924.

Micropoecilia HuBBs 1926: pré-employé, syn. de Recepoecilia W. 1953.

Mohanga BOULENGER 1911.

Neofundulus MYERS 1924.

Neolebias STEINDACHNER 1894.

Nothobranchius PETERS 1868.

Oryzias Jordan et SNYDER 1906.

Oxyzygonectes FOWLER 1916.

Pachypanchax MYERS 1933.

Panchax Cuvier et VAlenciennes 1846: syn. d'Aplocheilus M. C. 1839.

Pantanodon MYERS 1955.

Plataplochilus AHL 1928.

Platypanchax AHL 1928.

Potamophylax MYERS et DE CARVALHO 1955.

Prinodon RAFINESQUe 1815 : syn. de Cyprinodon L. 1803.

Procatopus Boulenger 1904.

Profundulus HuBBs 1925.

Pterolebias Garman 1895.

Quintana HuBBs 1934.

Rachovia MYERS 1927.

Recepoecilia WHITLEY 1953.

Rivulichthys MYERS 1927.

Rivulus POEY 1860.

Simpsonichthys de CARVALHO 1959. Tellia Gervais 1853: syn. d'Aphanius N. 1827.

Tlaloc Alvarez et Carranza 1951: syn. de Profundulus H. 1925.

Trifarcius Poey 1860: syn. de $\boldsymbol{C} \boldsymbol{y}$ prinodon L. 1803.
Trigonectes MYERS 1927.

Valencia MYERS 1928.

Vomerivulus FOWLER 1945: s./g. de Rivulus P. 1860.

Xenisma Jordan 1877: s./g. de Fundulus L. 1803.

Xenodexia HubBs 1950.

Zygonectes AGASSIZ 1853.

FAMILLE DES ORESTIIDAE

Empetrichthys GILBERT 1893.

Orestias CUVIER et VALENCIENNES 1846.

FAM. DES CHARACODONTIDAE

Allodontichthys HuBBS et TURNER 1939.

Characodon GÜNTHER 1866.

Lermichthys HUBBS 1926.

Neoophorus HubBs et TURner 1939.

Neotoca HubBs et Turner 1939.

Xenotoca HubBs et TURner 1939.

Zoogoneticus MEEK 1902.

\section{FAMILLE DES GOODEIDAE}

Alloophorus HubBs et TURner 1939. Allotoca HubBs et Turner 1939.

Ataeniobius HubBs et TURner 1939.

Balsadichthys HuвBs 1926.

Chapalichthys MEEK 1902.

Dactylophallus RIVERo et RIVAS 1944.

Girardinichthys BLEEKER 1860.

Goodea JoRDAN 1879.

Limnurgus GüNTHER 1866: syn. de Girardinichthys B. 1860.

Ollentodon HUBBS et TURNER 1939. Skiffia MEEK 1902.

Xenendum Jordan et SNYDER 1899.

Xenoophorus HubBs et TURNER 1939.

FAMILLE DES POECILIIDAE

(Top-minnows)

Acropoecilia HILGENDORF 1889. 
Alazon Gistel 1848: syn. de Poecilia B. et S. 1801.

Alfaro MeEK 1912.

Allodontium RIVERo et RIVAS 1944. Allogambusia HuBBs 1925.

Alloheterandria HuBBs 1925.

Allopoecilia HuBBs 1925.

Arthrophallus HuBBs 1926: s./g. d'Heterandria A. 1853.

Aulophallus HuвBs 1926.

Belonesox KNER 1859.

Brachyrhaphis REGAN 1913.

Cnestrodon Garman 1895.

Curtipenis RIVAS et MYERS 1950.

Cynodonichthys MEEK 1904.

Darienichthys HuBBs 1925.

Diphyacantha HENN 1916.

Furcipenis HubBs 1931: syn. d'Alfaro M. 1912.

Gambusia POEY 1854.

Girardinus POEY 1854: syn. d'Heterandria A. 1853.

Glaridichthys GARMAN 1896.

Glaridodon GARMAN 1895: pré-employé, syn. de Glaridichthys G. 1896.

Gulapinnus LANGER 1913.

Hemixiphophorus BLEEKER 1859 : syn. de Gambusia P. 1854.

Heterandria AgAssIZ 1853.

Heterophallina HuBBS 1926: s./g. de Gambusia P. 1854

Heterophallus REGAN 1914.

Ilyodon EIGENMANN 1907.

Leptorhaphis REGAN 1913.

Limia Poey 1854: syn. de Poecilia B. et S. 1801.

Limia Poey 1861: syn. de Poecilia B. et S. 1801.

Microphallus HERRE 1926.

Micropoecilia HuBBS 1926.

Mollienesia LE SUEUR 1821.

Neoheterandria HENN 1916.

Neopoecilia HuBBs 1925.

Palosoma DEAN : errat. pro Petalosoma R. 1908.

Pamphoria Regan 1913.
Pamphorichthys REgAN 1913.

Panamichthys HuBBs 1925.

Paragambusia MeEK 1904.

Parapoecilia HuBBS 1925.

Petalosoma REgAN 1908: pré-employé, syn. d'Alfaro M. 1912.

Petalurichthys REgAN 1912: syn. d'Alfaro M. 1912.

Phallatorhynchus HENN 1916.

Phallichthys HuBBS 1925.

Phalloceros EIGENMANN 1907.

Phalloptychus EigenmanN 1907.

Phallotorynus HENN 1916.

Platypoecilus GÜNTHER 1866: s./g. de Xiphophorus H. 1848.

Plectrophallus FoWLER 1932: s./g. de Panamichthys H. 1925.

Poecilia Bloch et SchneIder 1801.

Poecilistes HubBs 1926.

Poecilodes STEINDACHNER 1863: syn. de Pseudoxiphophorus B. 1859.

Poecilopsis REgAN 1913.

Priapella REgAN 1913.

Priapichthys REgAN 1913.

Pseudopoecilia REgAN 1913.

Pseudoxiphophorus BLEEKER 1859.

Psychropoecilia MYERS 1935: s./g. de Mollienisia L. S. 1821.

Schizophallus HuBBs 1926: s./g. de Gambusia P. 1854.

Tomeureus EIGENMANN 1909.

Toxus EigenmanN 1903.

Trigonophallus HuBBs 1926.

Xenophallus HuBBs 1925.

Xenotaenia TURNER 1946.

Xiphophorus HECKEL 1848.

\section{FAMILLE DES FITZROYIDAE}

Fitzroyia GüNTHER 1866.

Jenynsia GüNTHER 1866: syn. de Fitzroyia G. 1866.

\section{FAMILLE DES ANABLEPIDAE (Quatro Ojos)}

Anableps Gronow 1763 : non valide. 
Anableps (G.) Scopoli 1777.

Anableps (G.) BLOCH et ScHNeIDER 1801: syn. d'Anableps (G.) S. 1777.

Peltatetraops FoWLER 1931: s./g. d'Anableps (G.) S. 1777.

FAM. DES ADRIANICHTHYIDAE

Adrianichthys WeBER 1913.

Xenopoecilus Regan 1911.

\section{Ordre des SYNGNATHIFORMES}

FAM. DES SOLENOSTOMIDAE

$(=$ Solenostomatidae

$=$ Solenichthyidae)

Solenichthus BLEEKER 1865: syn. de Solenostomus L. 1803.

Solenichthys BLEEKER 1865: vide Solenichthus B. 1865.

Solenostoma RAFINESQUE : Solenostomus L. 1803.

Solenostomatichthys BLEEKER 1873 : syn. de Solenostomus L. 1803.

Solenostomus DE LACÉPÈDE 1803.

FAMILLE DES SYNGNATHIDAE (= Hippocampidae)

(Hippocampes ; Pipe-fishes or sea-horses)

Acanthognathus DUNCKer 1912: préemployé, syn. de Dunckerocampus W. 1933.

Acentronura KaUP 1853.

Acestra JARDINE 1831: syn. de Nerophis R. 1810.

Acmonotus PhILIPPI 1896: syn. de Leptonotus K. 1853.

Acus Müller 1767: non valide.

Acus Valmont 1791: non valide, syn. de Syngnathus L. 1758.

Acus Swainson 1839: syn. de Nerophis R. 1810.

Aphelikturus PARR 1930.

Aphelichthys COPE 1893.
Anarchopterus HuBrs 1935.

Apterygocampus WEBER 1913.

Atelurus DUMÉRIL 1870.

Belonichthys PETERS 1868.

Bhanotia HORA 1926.

Bhanotichthys PARR 1930: s./g. d'Hippichthys B. 1849.

Bombonia HERRE 1947.

Bry $\boldsymbol{x}$ Herald 1940.

Bulbonaricus HERALD 1954: s./g. d'Ichthyocampus K. 1853.

Campichthys WhITLEY 1931: s./g. de Festucalex W. 1931.

Castelnauina FOIVLER 1907.

Choeroichthys KaUP 1853.

Coclonotus: errat. pro Coelonotus P. 1855.

Coelonotus Peters 1855 : syn. d'Hemithylacus K. 1856.

Corythoichthys KAUP 1853: syn. d'Hippichthys B. 1849.

Corythroichthys: vide Corythoichthys K. 1853.

Dermatostethus GILL 1862.

Doryichthys KAUP 1853.

Doryrhamphus KAUP 1853.

Dunckerocampus WHITLEY 1933.

Entelurus DumériL 1870: syn. de Nerophis R. 1810.

Farlapiscis WHITLEY 1931.

Festucalex WhITLEY 1931.

Filicampus WHITLEY 1950.

Gasterotokeus HECKEL 1853: syn. de Syngnathoides B. 1851.

Halicampoides FOWLER 1956.

Halicampus KAUP 1853.

Haliichthys GRAY 1859.

Hemimarsupium KAUP 1853: syn. d'Hemithylacus K. 1856.

Hemithylacus KAUP 1856.

Hippichthys BLEEKER 1849.

Hippocampus RAFINESQUE 1810.

Hippocampus LEACH 1814: syn. d'Hippocampus R. 1810.

Hippocampus CUVIER 1817: syn. d'Hippocampus R. 1810.

Hippohystrix WHITLEY 1940. 
Histiogamphelus Mc CULLOCH 1914.

Homolenus RAFINESQUE 1815: non valide.

Hymenolomus DUMÉRIL 1870: syn. de Protocampus G. 1870.

Hypselognathus WhITLEY 1950.

Ichthyocampus KAUP 1853.

Idiotropiscis WHITLEY 1947.

Jamsus GINSB URG 1937: s./g. d'Hippocampus R. 1810.

Kaupus Whitley 1952: s./g. de Leptonotus K. 1853.

Larvicampus WHITLEY 1950.

Leptoichthys KAUP 1853.

Leptonotus KAUP 1853.

Lissocampus WaIte et Hale 1921.

Macleayina FOWLER 1907.

Maroubra Whitley 1950.

Micrognathus DUNCKER 1912.

Microphis KAUP 1853.

Mitotichthys WHITLEY 1950.

Nannocampichthys MORA et MUKERII 1936.

Nannocampus GüNTHER 1870.

Nematostoma EICHEWALD 1831 : syn. de Nerophis R. 1810.

Nerophis RAFINESQUE 1810.

Nigracus WhitLey 1953: s./g. de Stigmatopora K. 1853.

Novacampus WhITLEY 1955.

Oostethus HuвBs 1929.

Osphyolax COPE 1875: syn. de Nerophis $\mathrm{R} .1810$.

Oxyleyna WhITLEY 1937.

Parabelonichthys FOWLER 1943.

Paramicrophis KLAUSEWITZ 1955.

Parasyngnathus WhITLEY 1943.

Penetropteryx LUNEL 1881.

Peromere DumérIL 1856: non valide, syn. de Typhlinus R. 1815.

Phanerotokeus DUNCKER 1944.

Phycodurus GILL 1896.

Phyllophorus RAFINESQUE 1815: non valide.

Phyllopteryx SWAINSON 1839.
Pipetella Whrtley 1951: syn. de Nigracus W. 1953.

Pipettella WhrtLey 1951: syn. de Nigracus W. 1953.

Pristidoryrhamphus FOWLER 1945.

Protocampus GüNTHER 1870.

Pseudophallus HeRALD 1940.

Pseudosyngnathus KNER 1863.

Pterure DumÉrIL 1856: non valide, syn. de Nerophis R. 1810.

Pugnaso Whitley 1950.

Runcinatus WhItLeY 1929.

Scyphius Risso 1826: syn. de Nerophis R. 1810.

Simosyngnathus FOWLER 1940.

Siokunichthys HERALD 1954.

Siphonostoma Kaup 1856: syn. de Typhlinus R. 1815.

Siphonostomus Kaup 1856: syn. de Typhlinus R. 1815.

Siphostoma RAFINESQUE 1810: syn. de Syngnathus L. 1758.

Solegnathus SWAINSON 1839.

Solenognathus BLEEKER 1856: syn. de Syngnathoides B. 1851.

Stigmatophora : errat. pro Stigmatopora K. 1853.

Stigmatopora KaUP 1853.

Stipecampus WhITLEY 1950.

Syngnathoides BLEEKER 1851.

Syngnathus LINNÉ 1758.

Syrictes JoRDAN et EVERMANN 1927.

Tiphlinus RAFINESQUE 1815: vide Typhlinus R. 1815.

Trachyrhamphus KAUN 1853.

Typhle RAFINESQUE 1810: pré-employé, syn. de Typhlinus R. 1815.

Typhlinus RAFINESQUe 1815.

Typhlus (BIBRON) BLEEKER 1870 : non valide.

Typhlus (BIBRON) DUMÉRII 1870. syn. d'Ichthyocampus K. 1853

Urocampus GüNTHER 1870.

Vanacampus WHITLEY 1951: s./g. de Parasyngnathus W. 1943.

Yozia Jordan et SNYDER 1901. 


\section{Ordre des GADIFORMFS}

\section{FAM. DES MACROUROIDIDAE}

Macrouroides SMITH et RADCLIFFE 1912.

Squalogadus Gilbert et HubBS 1916.

FAMILLE DES MACROURIDAE (= Macruridae

$=$ Coryphuenoididae)

(Queues-de-rat, grenadiers; Rat-tails) Abyssicola GoODE et BEAN 1895.

Albatrossia JORDAN et GILBERT 1898.

Ateleobrachium GILBERT et BURKE 1912: larve de Macrourus B. 1786.

Atherodon Gilbert et Hubbs 1920.

Bathygadus GüNTHER 1878.

Bogoslovius JORL:AN et EVERMANN 1898.

Cariburus PARR 1946.

Cetonurus GüNTHER 1887.

Chalinura GoODE et BEAN 1882: syn. de Coryphaenoides G. 1761.

Chalinurus GüNTHER 1887: emend. pro Chalinura G. et B. 1882 .

Chalmina GoODE et BEAN 1882 : préemployé, syn. de Fuyangia W. 1931.

Coccolus (Kaup) Bonaparte 1844 : syn. de Krohnius C. 1844.

Coelocephalus GILBERT et CRAMER 1897 : pré-employé, syn. de $\boldsymbol{M a}$ taeocephalus B. 1899.

Coelorhynchus GIORNA 1805.

Coryphaenoides GUNNER 1761.

Cynomacrurus Dollo 1909.

Dolloa Jordan 1900.

Echinomacrurus Roule 1916.

Fuyangia WHITLEY 1931.

Gadomus REGAN 1903.

Garichthys WhITLEY 1934.

Grenurus PARR 1946.

Hemimacrurus FRASER-BRUNNER 1935.
Hymenocephalus GIGLIOLI et IsSEL 1884.

Hymenogadus GILBERT et HUBBS 1920.

Hyomacrurus Gilbert et HusBs 1920.

Hyostomus Gilbert et HUBBS 1920. Krohnius Cocco 1844 : larve de Coelorhynchus G. 1805.

Lepidoleprus RIsso 1810: syn. de Trachyrincus G. 1805.

Lepidorhynchus BLEEKER 1879.

Lepidosoina SwaINSON 1839: errat. pro Lepidoleprus R. 1810.

Lepturus GRONOW 1854: syn. de Coryphaenoides G. 1761.

Lionurus GÜNTHER 1887.

Lucigadella GILBERT et HUBBs 1920.

Lucigadus GILBERT et HubBs 1920.

Lycondes GILCHRIST 1923.

Lyconodes GILCHRIST 1922.

Lyconus GüNTHER 1887.

Macrourus BLOCH 1786.

Macruronus GÜNTHER 1872.

Macruroplus BLEEKER 1874.

Macrurus SCHNEIDER 1801: emend. pro Macrourus B. 1786.

Malacocephalus GÜNTHER 1862.

Mataeocephalus BERG 1899.

Melanobranchus REGAN 1903.

Moseleya GoOdE et BEAN 1895 : préemloyé, syn. de Dolloa J. 1900.

Mystaconurus GüNTHER 1887: syn. d'Hymenocephalus G. et I. 1884.

Nematonurus GüNTHER 1887.

Nezumia Jordan et GIIBERT 1904.

Odontomacrurus NORMAN 1939.

Optonurus GüNTHER 1887

Oxycephas RAFINESQUE 1810: syn. de Trachyrincus G. 1805.

Oxygadus Gilbert et HubBs 1920.

Oxymacrurus BLEEKER 1874.

Papyrocephalus Gilbert et HubBs 1920.

Paramacrurus BLEEKER 1874.

Pawnurus PARR 1946: s./g. de Malacocephalus G. 1862. 
Phalacomacrurus MAUL et KOEFOED 1950.

Quincuncia Gilbert et HubBs 1920.

Regania Jordan et GILBERT 1904.

Sphagemacrurus FOWLER 1925: s./g. de Macrurus B. 1786.

Steindachnerella EIGENMANN 1897: syn. de Steindachneria G. et B. 1888 .

Steindachneria GOODE et BEAN 1888.

Trachonurus GÜNTHER 1887.

Trachyrhynchus BONAPARTE 1841: emend. pro Trachyrincus G. 1805.

Trachyrincus GIORNA 1805.

Ventrifossa GILBERT et HubBs 1920.

Xenocephalus KaUP 1858.

FAM. DES BREGMACEROTIDAE Asthenurus Tickell 1865: syn. de Bregmaceros (C.) T. 1840.

Auchenoceros GüNTHER 1889.

Bregmaceros (CANTOR) THOMPSON 1840.

Calloptilum RICHARDSON 1844 : préemployé, syn. de Bregmaceros (C.) T. 1840 .

Calloptilum Hutron 1872: pré-employé, syn. d'Auchenoceros G. 1889.

FAM. DES GAIDROPSARIDAE

(Motèles ou Mustèles; Rocklings)

Ciliata Couch 1832.

Couchia Thompson 1856: larve de Ciliata C. 1832.

Dropsarus RAFINESQUe 1815: syn. de Gaidropsarus R. 1810.

Enchelyopus BLOCH et SCHNEIDER 1801.

Gaidropsarus RAFINESQUE 1810.

Gaidrosparus : errat. pro Gaidropsarus $\mathrm{R} .1810$.

Hypsiptera GüNTHER 1860: larve de Gaidropsaridae sp.

Molvella Kaup 1858: syn. de Ciliata C. 1832 .
Motella Cuvier 1829: syn. de Gaidropsarus R. 1818.

Mustela (CUVIER) OKEN 1817: préemployé, syn. de Gaidropsarus R. 1810.

Onogadus DE BUEN 1935.

Onos Risso 1826: syn. de Gaidropsarus R. 1810.

Rhinonemus GILL 1863.

FAMILLE DES MERLUCCIIDAE (Merlus et Merlans; Hakes)

Epicopus GüNTHER 1860: syn. de Merluccius R. 1810.

Homalopomus GIRARD 1856: syn. de Merluccius R. 1810.

Hydronus Minding 1832: syn. de Merluccius R. 1810.

Merlangius GeOFFroY-ST-HILAIRE 1767: non valide.

Merlangus RAFINESQUE 1810.

Merluccius RAFINESQUE 1810.

Merlucius Gronow 1854: syn. de Merluccius R. 1810.

Merlus GUICHENOT 1848: syn. de Merluccius R. 1810.

Onus RAFINESQUE 1810: syn. de Merluccius R. 1810.

Promerluccius BOGHACHEV 1938.

Stomodon Mrtchill 1814: syn. de Merluccius R. 1810.

Trachinoides BORODIN 1934: syn. de Merluccius R. 1810.

\section{FAMILLE DES GADIDAE}

(Morue, Haddock. Tacaud et Lieu ; Cod-fishes)

Aeglefinus Malm 1877: syn. de Melanogrammus G. 1862.

Algoa Castelnau 1861.

Arctogadus DrJagin 1932.

Austrophycis OGILBY 1897.

Boreogadus GÜNTHER 1862.

Brachygadus GILL 1862: syn. de Trisopterus R. 1814.

Brachygadus GILL 1863: syn. de Trisopterus R. 1814.

Brachymesistius GILL 1863: errat. pro Micromesistius G. 1863. 
Brosme (CUVIER) OKEN 1817.

Brosmiculus VAILlant 1888.

Brosmius Cuvier 1829: syn. de Brosme (C.) O. 1817.

Brosmus Stark 1828: syn. de Brosme (C.) O. 1817.

Callarias KLEIN 1777 : non valide.

Cephus Swainson 1839: syn. de Gadus L. 1758.

Cerdo Gistel 1848: syn. de Gadus L. 1758.

Eleginus FISCHER 1813

Emphycus JORDAN et EVERMANN 1898: syn. de Phycis R. 1793.

Euclichthys Mc Culloch 1926.

Ficis S. D. W. 1837 : syn. de Phycis R. 1793.

Gadella Lowe 1843.

Gadiculus GUICHENOT 1850.

Gadopsis DE FILIPPI 1855: pré-employé, syn. de Verater J. 1919.

Gadus LINNÉ 1758.

Gargilius (JENSEN) SCHMIDT 1906: non valide.

Guttigadus TAKI 1953 : s./g. de Laemonema G. 1862.

Halargyreus GüNTHER 1862.

Hypsirhynchus FAcciola 1884 : préemployé, syn. de Rhynchogadus T. 1952.

Laemonema GüNTHER 1862.

Laemonemodus GILCHRIST 1903.

Leptogadus Gill 1863: syn. de Gadiculus G. 1850.

Leptophycis GARMAN 1899.

Lota (CUVIER) OKEN 1817.

Lotella KAUP 1856.

Lotta Risso 1826 : non valide, syn. de Lota (C.) O. 1817.

Melanogrammus GILL 1862.

Melanosoma GILCHRIST 1902.

Melanonus GÜNTHER 1878.

Merlangus (CUVIER) OKEN 1817 : syn. de Gadus L. 1758.

Merlinus Agassiz 1845: non valide.

Microgadus GILL 1863.

Microlepidium Garman 1899.

Micromesistius GILL 1863.

Molua (RONDELeT) SMITH 1893 : syn. de Molva F. 1828.
Molva Fleming 1828.

Morhua Fleming 1828: syn. de Gadus L. 1758.

Morrhua (CUVIER) OKEN 1817: syn. de Gadus L. 1758.

Morua Risso 1826: syn. de Trisopterus R. 1814.

Odontogadus GILL 1863.

Olssonichthys FowLeR 1959: syn. de Khynchogadus T. 1952.

Phocaegadus JENSEN 1949: syn. d'Arctogadus D. 1932.

Phycis RösE 1793.

Phycis Bloch et SchNeIDER 1801: syn. de Phycis R. 1793.

Phycis RAFINESQUE 1810: pré-employé, syn. de Phycis R. 1793.

Physiculus KAUP 1858.

Pleurogadus (KAUP) JORDAN 1885: syn. d'Eleginus FISCHER 1813.

Podonema RASS 1955.

Pollachius (NILsson) Bonaparte 1845.

Pseudophycis GüNTHER 1865.

Pteridium FILIPPI et VERANY 1859: pré-employé, syn. de Verater J. 1919.

Raniceps (CUVIER) OKEN 1817.

Rhynchogadus TORTONESE 1952.

Salilota GüNTHER 1887.

Strinsia RAFINESQUE 1810.

Sympodoichthys FAcciola 1888.

Theragra LUCAS 1897.

Tilesia Swatson 1839: pré-employé, syn. d'Eleginus F. 1813.

Tripterophycis BOULENGER 1902.

Trisopterus RAFINESQUE 1814.

Uraleptus Costa 1846.

Uraleptus Costa 1858: syn. de $\boldsymbol{G a}$ della L. 1843.

Urophycis GILL 1863.

Verater JORDAN 1919.

\section{FAMILLE DES MORIDAE}

Antimora GüNTHER 1878.

Asellus VALENCIENNES 1844 : syn. de Mora R. 1826.

Gastronemus (Cocco) Bonaparte 1845: pré-employé, syn. de Mora R. 1826. 
Haloporphyrus GüNTHER 1862.

Lepidion SwaINSON 1839: pré-employé, syn. de Haloporphyrus G. 1862 .

Mora Risso 1826.

Pharopteryx RüPPELL 1852: syn. de Mora R. 1826.

Tinimogaster BONAPARTE 1845: non valide, syn. de Mora R. 1826.

\section{FAM. DES MURAENOLEPIDAE}

Muraenolepis GÜNTHER 1880.

FAM. DES ERETMOPHORIDAE

Eretmophorus GIGLIOLI 1889.

Hypsirhynchus FACCIOLA 1884.

\section{Ordre des LAMPRIDIFORMES}

\section{FAMILLE DES VELIFERIDAE}

Velifer Temminck et Schlegel 1850.

\section{FAMILLE DES LAMPRIDIDAE}

(= Lampridae) (Opah; Moon-fishes)

Chrysotosus DE LACÉPÈDE 1803: syn. de Lampris R. 1799.

Echemythes GISTEL 1848: syn. de Lampris R. 1799.

Lampris RETZIUS 1799.

\section{FAM. DES TRACHIPTERIDAE}

(Flambe, Argentin, Ruban-de-mer ; King of the Herring)

Agrostichthys PHILlipPS 1924.

Argyctius RAFINESQUE 1810: syn. de Trachipterus G. 1770 .

Bogmarus BLOCH et SCHNEIDER 1801: syn. de Trachipterus G. 1770. Gymnogaster BRüNNICH 1788: syn. de Trachipterus G. 1770.

Nemochirus RAFINESQUE 1815: syn. de Trachipterus G. 1770.

Nemotherus Costa 1834: syn. de Trachipterus G. 1770.

Trachipterus GOUAN 1770.
Trachypterus SCHNEIDER 1801 : emend. pro Trachipterus G. 1770.

Vogmarus ReID 1849: syn. de Trachipterus G. 1770.

FAMILLE DES REGALECIDAE (Roi-des-harengs ; Oar-fishes or Sea-serpents)

Cephalaspis RAFINESQUE 1810: syn. de Regalecus B. 1771.

Cephalepis RAFINESQUE 1810 : syn. de Regalecus B. 1771.

Epidesmus Ranzani 1810: syn. de Regalecus B. 1771.

Gymnetrus BLOCH 1788: syn. de Regalecus B. 1771.

Regalecus BRüNNICH 1771 .

Regalecus ascanius 1788: syn. de Regalecus B. 1771.

Xiphichthys SwaInSON 1839 : syn. de Regalecus B. 1771.

Xypterus RAFINESQue 1810: syn de Regalecus B. 1771.

\section{FAMILLE DES LOPHOTIDAE}

Eumecichthys REGAN 1907.

Leptopus RAFINESQUE 1814: syn. de Lophotes G. 1805.

Lophotes GIORNA 1805.

Lophotopsis BARNARD 1925.

Podoleptus RAFINESQue 1815: syn. de Lophotes G. 1805.

Regilophotes WHITLEY 1933.

FAMILLE DES STYLEPHORIDAE

Nemochirus RAFINESQUE 1815: non valide, syn. de Stylephorus S. 1891.

Stylephorus SHaw 1891.

Stylophorus BERTHOLD 1827 : emend. pro Stylephorus S. 1891

FAMILLE DES GIGANTURIDAE Gigantura BRAUER 1901. 


\section{Ordre des BERYCIFORMES}

FAMILLE DES POLYMIXIIDAE

Dinemus Poey 1860 : syn. de Polymixia L. 1838.

Nemobrama VALENCIENNES 1844: syn. de Polymixia L. 1838.

Polymixia Lowe 1838.

\section{FAMILLE DES BERYCIDAE}

Actinoberyx Roule 1923.

Austroberyx Mc Culloch 1911 : syn. de Trachichthodes G. 1903.

Beryx CUVIER 1829.

Centroberyx GILL 1862.

Dictynopterus WHITLEY 1953.

Hoplopteryx REgAN 1911: pré-employé, syn. de Centroberyx G. 1862.

Lobopterus KRAMBERGER 1895: syn. de Dictynopterus W. 1953.

Trachichthodes GILCHRIST 1903.

FAM. DES OSTRACOBERYCIDAE

Ostracoberyx FOWLER 1934.

FAMILLE DES ANOMALOPIDAE

Anomalops KNER 1868.

Heterophthalmus BLEEKER 1856: préemployé, syn. d'Anomalops K. 1868.

Kryptophanaron SILVESTER et FowLER 1926.

Photoblepharon WEBER 1902.

FAM. DES TRACHYBERYCIDAE

Trachyberyx ROULE 1929.

\section{FAMILLE DES DIRETMIDAE}

Campbellina FOWLER 1939.

Crossochir HuBBs 1933.

Diretmus JoHnson 1863.

Discus Campbell 1879: syn. de Diretmus J. 1863.
Paradiretmus Whitley 1950.

FAMILLE DES CARISTIIDAE

Caristius GILl et SMITH 1905.

Platyberyx ZugMAYER 1911.

\section{FAM. DES TRACHICHTHYIDAE}

Aulohoplostethus FOWLER 1938: s./g. d'Hoplostethus C. et V. 1829.

Aulotrachichthys FOWLER 1938: s./g. de Trachichthys S. 1799.

Gephyroberyx BOULENGER 1902.

Hoplostethus CUVIER et VALENCIENNES 1829.

Leiogaster WEBER 1913.

Optivus WhitLey 1947.

Paratrachichthys WAITE 1899.

Trachichthys SHAW 1799.

\section{FAM. DES MONOCENTRIDAE}

(Pine-cone fishes)

Cleidopus De VIs 1883 : syn. de Mo. nocentris B. et $\mathrm{S}$. 1801.

Ericius Tilesius 1809: syn. de Monocentris B. et $S .1801$.

Lepicantha RAFINESQUE $1815^{\circ}$ syn. de Monocentris B. et S. 1801.

Lepisacanthus DE LACÉPÈDE 1802: syn. de Monocentris B. et S. 1801.

Monocentris BLOCH et SCHNEIDER 1801.

FAMILLE DES HOLOCENTRIDAE (Soldier-fishes)

Adioryx STARKS 1908.

Beanea STEINDACHNER 1902.

Beloholocentrus FOWLER 1944.

Cephalofarer WHITLEY 1933

Corniger Agassiz 1829.

Eritrinus PLUMIER : non valide. Erythrinus (P.) DE LACÉPÈDE 1803: non valide, syn. d'Holocentrus (G.) S. 1777. 
Faremusca Whitley 1933 : s./g. de Holocentrus (G.) S. 1777.

Farer ForsKaL 1775: non valide.

Flammeo JoRDAN et EVERMANN 1898.

Harpage DEVIS 1884.

Holocentrum: vide Holocentrus (G.) S. 1777.

Holocentrus GroNow 1763 : non valide.

Holocentrus (G.) Scopoli 1777.

Holotrachys GÜNTHER 1873.

Hovella OGILby 1898.

Kurtaflammeo WhITLEY 1933 : s./g. de Holocentrus (G.) S. 1777.

Myripristis CUVIER 1829.

Neomyripristis de CASTELnaU 1873.

Neoniphon de CASTELNAU 1875.

Osteichthys: vide Ostichthys (L.) J. et E. 1896.

Ostichthys (LANGSDORFF) CUVIER et VALENCIENNES 1829: non valide.

Ostichthys (LANGSDORFF) JORDAN et EVERMANN 1896.

Plectrypops GILL 1862.

Rhamphoberyx GILL 1863: larve de Myripristis C. 1829.

Rhinoberyx GILL 1862: larve d'Holocentrus (G.) S. 1777.

Rhynchichthys CUVIER et VALENCIENNES 1831: larve d'Holocentrus (G.) S. 1777.

Sargocentron FOWLER 1904.

FAM. DES SOROSICHTHYIDAE

Sorosichthys WHITLEY 1945.

FAM. DES GIBBERICHTHYIDAE

Gibberichthys PARR 1933.

FAM. DES KORSOGASTERIDAE Korsogaster PARR 1933.

\section{Ordre des STEPHANOBERYCIFORMES}

FAMILLE DES RONDELETIIDAE

Rondeletia GoODE et BEAN 1894.

FAMILLE DES BARBOURISIIDAE

Barbourisia PARR 1945.

FAMILLE DES CETOMIMIDAE

Cetomimoides KOEFOED 1955.

Cetomimus Goode et BeAN 1894.

Cetostoma Zugmayer 1913.

Ditropichthys PARR 1934.

Gyrinomimus PARR 1934.

Pelecinomimus GILCHRIST 1922: syn. de Cetomimus G. et B. 1894.

Psapharocetus HARRY 1952: s./g. de Cetomimus G. et B. 1894.

Vitiaziella RASs 1955.

\section{FAMILLE DES \\ STEPHANOBERYCIDAE}

Acanthochaenus GILL 1884.

Malacosarcus GÜNTHER 1887.

Stephanoberyx GILL 1883.

FAM. DES MELAMPHAEIDAE

Anoplogaster GÜNTHER 1859.

Caulolepis GILL 1883.

Lophocephalus OsoRIo 1909: syn. de Melamphaes G. 1859.

Melamphaes GÜNTHER 1859.

Metopias Lowe 1843: pré-employé, syn. de Melamphaes G. 1859.

Plectromus GILL 1883.

Poromitra GoOde et BEAN 1881.

Promitrella ZugMaYer 1911.

Scopeloberyx ZugmaYer 1911.

Scopelogadus VAILlant 1888: syn. de Melamphaes G. 1859. 
Ordre des ZEIFORMES

FAMILLE DES ZEIDAE

(Saint-Pierre, Poule de mer, Jean Doré ; John-Dories)

Allocyttus MC Culloch 1914. Capromimus GILL 1893.

Cyttoides SмIтн 1946.

Cyttoidops SMITH 1949.

Cyttomimus GILbert 1905.

Cyttopsis GILL 1862.

Cyttosoma Gilchrist 1903.

Cyttula WeBer 1913.

Cyttus GüNTHer 1861.

Macrurocyttus FOWLER 1934.

Neocyttus GILCHRIST 1906.

Oreosoma CUVIER 1829.

Paracyttopsis GILCHRIST et BONDE 1924.

Parazenopsis Cligny 1909.

Perigrammatolepis GEINITZ 1868.

Prionolepis SMITH 1931.

Pseudocyttus GILCHRIST 1906.

Rhombocyttus GILL 1893.

Stethopristes GILBERT 1905.

Xenocyttus ABE 1957.

Zen JoRDAN 1903.

Zenion Jordan et EVERMANN 1896.

Zenopsis GILL 1862.

Zeus LINNÉ 1758.

\section{FAMILLE DES \\ GRAMMICOLEPIDIDAE}

Grammicolepis POEY 1873.

Vesposus JORDAN 1921.

Xenolepidichthys GILCHRIST 1922.

FAMILLE DES CAPROIDAE

Capros de Lacépède 1803.

Crassispinus Maul 1948.
FAMILLE DES ANTIGONIIDAE Acanthagonia OGILBY 1918.

Antigonia Lowe 1843.

Caprophonus Müller et Troschel 1849 : syn. d'Antigonia L. 1843. Hypsinotus TemmincK et Schlegel 1844 : syn. d'Antigonia L. 1843.

Ordre des CORYPHAENIFORMES

FAMILLE DES BRAMIDAE

(Brèmes-de-mer, Castagnoles ;

Sea-breams)

Argo DöDerlein 1883 : syn. de $\boldsymbol{T} \boldsymbol{a}$ ractes L. 1843.

Brama Bloch et SCHNEIDER 1801.

Collybus SNYDER 1904.

Eumegistus JoRdAN et JoRdan 1923.

Lepidotus: errat. pro Lepodus $\mathbf{R}$. 1810.

Lepodus RAFINESQue 1810: syn. de Brama B. et S. 1801.

Taractes LowE 1843.

Taractichthys MEAD et MAUL 1958: s./g. de Taractes L. 1843.

Tylometopon (VAN BEMMELEN) BLEEKER 1876: non valide, syn. de Brama B. et S. 1801.

FAMILLE DES STEINEGERIIDAE

Steinegeria JORDAN et EVERMANN 1886.

FAMILLE DES DIANIDAE

Astrodermus (Bonelli) CUVi E R 1829.

Diana Risso 1826.

Proctostegus NaRdo 1827: larve de Diana R. 1826.

FAMILLE DES ELEPHENORIDAE Elephenor JORDAN 1919. 
FAMILLE DES PTERACLIDAE

$$
(=\text { Pteraclididae) }
$$

Bentenia JORDAN et SNYDER 1901.

Centropholis HILGENDORF 1949: syn. de Pterycombus F. 1837.

Centropholoides SMITH 1949: syn. de Pterycombus F. 1837.

Oligopodes (RISso) CUVIER 1827.

Oligopodus DE LACÉPÈDE 1800: syn. de Pteraclis G. 1772.

Oligopus Risso 1826.

Pteraclidus RAFINESQUE 1815: syn. de Pteraclis G. 1772.

Pteraclis Gronow 1763: non valide.

Pteraclis Gronow 1772.

Pteridium Scopoli 1777.

Pterycombus FRIES 1837.

FAM. DES CORYPHAENIDAE

(Dauphins; Dolphins; Dorados)

Caranxomorus DE LACÉPÈdE 1802 : syn. de Coryphaena L. 1758.

Coryphaena LINNÉ 1758.

Coryphus (COMMERSON) DE LACÉPÈdE

1802: non valide.

Ectenias JoRDAN et THOMPSON 1914. Hippurus KLEIN 1779: non valide.

Lampugus Cuvier et VALENCIENNES 1833: syn. de Coryphaena L. 1758.

Lepimphis RAFINESQUE 1810: syn. de Coryphaena L. 1758.

\section{Ordre des PERCIFORMES}

- Beaucoup de familles devraient être ramenées au rang de sous-familles ou même tomber en synonymie. » (David Star JORDAN, 1923).

\section{Sous-ordre des PERCOIDEI}

FAMILLE DES SERRANIDAE

(Perches-de-mer, Barbiers ; Sea-Bass)

Acroperca MYERS 1933.

Altiserranus WhITLEY 1947.

Anogramma OGILby 1899: syn. de Callanthias L. 1839.

Anthias BLOCH 1792.

Anthiasicus GINSBURg 1952.

Aporops Schultz 1943.

Archeria Nichols 1950.

Atractoperca GILL 1861: syn. de Paralabrax G. 1856.

Aylopon RAFINESQue 1810: syn. de Anthias B. 1792.

Bathyanthias GüNTHER 1880.

Belonoperca Fowler et BEAN 1930. Brachyrhinus GILL 1862: pré-employé, syn. de Paranthias G. 1868.

Caesioperca de Castelnau 1872.

Callanthias Lowe 1839.

Callidulus FOWLER 1907.

Caprodon Temminck et Schlegel 1843.

Caribrhegma BREDER 1927.

Centropristes CUVIER 1829.

Centropristis: vide Centropristes C. 1829.

Chelidoperca BOULENGER 1895.

Chenia FOWLER 1939.

Chromanthias WHITLEY 1935.

Cookeolus Fowler 1929: s./g. d'Anthias B. 1792.

Colpognathus KLUNZINGER 1880 : pré-employé, syn. de Chenia F. 1959.

Cratinus STEINDACHNER 1878.

Creolus Jordan et GILBERT 1882: syn. de Paranthias G. 1868.

Dactylanthias BLEEKER 1873.

Deuteracanthus FOWLER 1944.

Diplectrum HolBRoOK 1855.

Dules Cuvier 1828: pré-employé, syn. de Eudulus F. 1907. 
Dulichthys BONAPARTE 1831.

Emmelanthias SмIтH 1955.

Entonanthias JORDAN et TANAKA 1927.

Epelytes EVERMANN et RADCLIFFE 1871: syn. de Pinguilabrum H. 1946.

Eudulus Fowler 1907: syn. de Dulichthys B. 1831.

Franzia JoRDAN et ThOMPSON 1914.

Fraudella WhITLEY 1935.

Giganthias KatAYAMA 1954.

Gilbertia JoRDAN et EIGENMANN 1890.

Gonioperca GILL 1862: syn. de Paralabrax G. 1856.

Grammatonotus GILBERT 1905.

Haliperca GILL 1862.

Hemianthias STEINDACHNER 1874.

Hepatus Röse 1793: pré-employé, syn. de Paracentropristis K. 1870.

Holanthias GüNTHER 1869.

Hypoplectrodes GILL 1862: nom. nud, syn. de Gilbertia J. et E. 1890.

Hypoplectrus GILL 1862.

Hyposerranus KLUNZINGER 1884.

Lacepedia de Caesioperca C. 1872. : syn.

Lepidoperca REGAN 1914.

Leptanthias TANAKA 1918.

Luzonichthys HERRE 1936.

Maccullochella WhITLEY 1929.

Malacocanthus : vide Malacanthus?.

Mentiperca GILL 1862.

Mirolabrichthys HERRE et MONTALBAN.

Naurua Whitley et Colefax 1938.

Neanthias Norman 1931: pré-employé, syn. de Novanthias W. 1937.

Nemanthias SMITH 1954.

Neoanthias DE CASTELNAU 1878: syn. de Caprodon T. et S. 1843.

Neoniphon DE VIS 1885: pré-employé.

Novanthias WHITLEY 1937.
Ocyanthias JORDAN et EVERMANN 1896.

Palsartia WhItLey 1943.

Paracentropristis KLUNZINGER 1870.

Paralabrax GIRARD 1856.

Paranthias GUICHENOT 1868.

Parasphenanthias GILCHRIST 1923 : syn. de Owstomia.

Parosphenanthias GILCHRIST 1922.

Percanthias TANAKA 1922.

Percaprionodes FOWLER 1944 : s./g. de Prionodes J. 1842.

Pingalla Whitley 1955.

Pinguilabrum Hildebrand 1946.

Planctanthias FOWLER 1935.

Plectranthias BLEEKER 1873.

Prionodes JeNYNS 1842.

Pronotogrammus GILL 1863.

Pseudanthias BLEEKER 1873.

Pseudocrenilabrus FOWLER 1933.

Pseudoserranus KLUnZINGer 1870.

Pteranthias WeBER 1913.

Rainfordia MC CULlOCH 1923.

Rhomboserranus FOWLER 1943.

Rhyacanthias JORDAN 1921.

Rosanthias TANAKA 1917.

Sacura Jordan et Richardson 1910.

Sayonara JoRdan et SEALE 1906.

Schistoperca FOWLER 1943.

Selenanthias TANAKA 1918.

Serranellus JORDAN et EIGENMANN 1890.

Serraniculus GINSBURg 1952.

Serranops REgAN 1914.

Serranus CUVIER 1817.

Serrihastaperca FOWLER 1944.

Sphenanthias WEBER 1913.

Spinipercina FOWLER 1944.

Stereolepidella WhITLEY 1954.

Stereolepis AYRES 1859.

Stereolepis CASIER : pré-employé, syn. de Stereolepidella W. 1954

Stereolepoides FOWLER 1923.

Symphysanodon BLEEKER 1878.

Tosanoides KAMOHARA 1953. 
Triloburus GiLl 1861 : syn. de Centropristes C. 1829.

Tulelepis Sмгтн 1954.

Xenanthias REgAN 1908.

Zalanthias JORDAN et RICHARDSON 1910.

\section{FAMILLE DES \\ NORMANICHTHYIDAE}

Normanichthys ClaRKe 1937.

FAM. DES ANISOCHROMIDAE Anisochromis.

FAM. DES STIGMATONOTIDAE

Stigmatonotus PETERS 1876.

FAMILLE DES EPINEPHELIDAE (Cerniers, Mérous; Sea-bass, Groupers ; Garrupas)

Acunthistius GILL 1862.

Acanthocephalus DöDERLEIN 1883: pré-employé, syn. de Doderleinnia S. 1883.

Actenolepis DyвowsKi 1872 : syn. de Siniperca G. 1862.

Aethaloperca FowLER 1904.

Alphestes Bloch et SCHNEIDER 1801. Anyperodon GüNTHER 1859.

Archoperca JoRDAN et EVERMANN 1896.

Aulacocephalus TeMmincK et SCHLEGEL 1842.

Bodianus BLOCH 1790.

Bodianus Cuvier et VAlenciennes : pré-employé, syn. de Cephalopholis B. et S. 1801.

Bostockia DE CASTELNAU 1873.

Bryttosus JORDAN et SNYDER 1900.

Centristhmus Garman 1899.

Centrogenys RICHARDSON 1842.

Cephalopholis BLOCH et SCHNEIDER 1801.

Cerna Bonaparte 1837 : syn. d'Epinephelus B. 1793.

Chorististium GILL 1862.
Chrysomelanus (PLUMIER) DE LACÉPÈDE 1803: non valide, syn. d'Epinephelus B. 1793.

Coreoperca HerzensteIn 1896.

Coreosiniperca FANG et CHONG 1933: s./g. de Siniperca G. 1862.

Coreospiniperca FANG et CHONG 1933: errat. pro Coreosiniperca F. et C. 1933.

Corusculus Jordan et SNYDER 1901 : syn. de Doderleinia S. 1883.

Cromileptes SwaInson 1839.

Ctenolates GüNTHER 1871.

Cugupuguacu CATESBY 1771: non valide.

Cynichthys SwaInson 1839: syn. d'Epinephelus B. 1793.

Daba FORSKAL 1775 : non valide, syn. d'Epinephelus B. 1793.

Dermatolepis GILL 1861.

Dinoperca Boulenger 1895.

Doderleinia STEINDACHNER 1883.

Eleutheractis COPE 1871: syn. de Rypticus C. 1829.

Ellerkeldia WHITLEY 1927.

Enneacentrus GILL 1865: syn. de Cephalopholis B. et S. 1801.

Enneistus JORDAN et EVERMANN 1896.

Epelytes EvermanN et RADCLIFFE 1917.

Epinephalus JoRDAN 1919 : errat. pro Epinephelus B. 1793.

Epinephelides OGILBy 1899.

Epinephelus BLOCH 1793.

Eteliscus JORDAN et SNYDER 1900: syn. de Doderleinia S. 1883.

Garrupa JORDAN et EIGENMANN 1890. Gennadius JoRdan et SEALE 1907 : syn. de Centrogenys R. 1842.

Gilbertella WaITE 1902: syn. d'Epinephelides O. 1899.

Gonioplectrus GILL 1862.

Grammistes BLOCH et SCHNEIDER 1801.

Grammistops SCHULTZ 1954.

Hectoria de Castelnau 1873: syn. de Polyprion (C.) O. 1817.

Helops BROWNe 1789: non valide. 
Hemilutjanus BLEEKER 1876: syn. de Pomodon B. 1895.

Homalogrystes AlleYNes et MACLEAY 1877.

Hyporthodus Gill 1862 : syn. d'Epinephelus B. 1793.

Itaiara VAILLANT et BocourT 1878: syn. de Promicrops D. 1868.

Labracopsis STEINDACHNER et DöDERLEIN 1883.

Labroperca GILL 1862: syn. d'Epinephelus B. 1793.

Lioperca GILl 1862: syn. de Dermatolepis G. 1861.

Liopropoma GILL 1861.

Louti ForSKaL 1775 : non valide.

Macquaria CUVIER et VALENCIENNES 1830.

Megaperca HILGENDORF 1878: syn. de Stereolepis A. 1858.

Menephorus POEY 1871.

Merou Bonaparte 1831 : syn. d'Epinephelus B. 1793.

Murrayia DE CASTELnAU 1872: syn. de Macquaria C. et V. 1830.

Mustelichthys TANAKA 1918.

Mycteroperca GILL 1862.

Myriodon BRISOUT DE BARNEVILLE 1847: syn. de Centrogenys R. 1842.

Notognidion RAfINesQue 1810.

Paschalestes GISTEL 1848: syn. de Macquaria C. et V. 1830.

Petrometopon GILL 1865.

Phaetonichthys BLEEKER 1876: syn. de Cephalopholis B. et S. 1801.

Pikea Steindachner 1874.

Plectroperca Peters 1865: syn. de Siniperca G. 1862.

Plectroplites GILL 1862.

Plectropoma : vide Plectropomus (C.) O. 1817.

Plectropomus (CUVIER) OKEN 1817.

Pleuroperca Fowlen et BEAN 1930: s./g. de Plectropomus (C.) O. 1817.

Pogonoperca GüNther 1859.

Polyprion (CUVIER) OKen 1817.

Pomodon Boulenger 1895.
Priacanthichthys DAY 1868: syn. d'Epinephelus B. 1793.

Promicrops DAY 1868.

Promicropterus GILL 1861.

Prospinus POEY 1861: syn. d'Alphestes B. et S. 1801 .

Pseudalphestes BOULENGER 1895.

Rhabdosebastes FowLER et BEAN 1922: syn. de Centrogenys R. 1842.

Rhypticus: vide Rypticus CUVIER 1829.

Riverina DE CASTELNAU 1872: syn. de Macquaria C. et V. 1830.

Rypticus CUVIER 1829.

Schistorus GILL 1862.

Serranichthys BLEEKER 1855: syn. de Cromileptes S. 1839.

Serranus Cuvier 1817: syn. d'E pinephelus B. 1793.

Siniperca GILL 1862.

Smecticus VALENCIENNES 1855: syn. de Rypticus C. 1829.

Stereolepis AYRES 1858.

Trachypoma GüNTHER 1859.

Trisopteropsis GILL 1865.

Uriphaeton SwaInson 1839: syn. de Cephalopholis B. et S. 1801.

Variola SwaInson 1839.

Vivero JoRdAN et EVERMANN 1927 : s./g. d'Epinephelus B. 1793.

Xystroperca JoRDAN et EVERMANN 1896.

Ypsigramma Schultz 1953: syn. de Choristium G. 1862.

\section{FAMILLE DES NIPHONIDAE}

Niphon Cuvier et Valenciennes 1828.

\section{FAMILLE DES OLIGORIDAE}

Homodemus DE VIS 1885: syn. d'Oligorus G. 1859.

Lateolabrax BLEEKER 1857.

Malakichthys DÖDERLEIN 1883.

Odontolabrax BLEEKER 1873.

Oligorus GüNTHER 1859. 
Perca-labrax Temminck et Schlegel 1842: non valide.

Percalabrax (T. et S.) Günther 1859. syn. de Lateolabrax G. 1857.

Satsuma SMITH et POPE 1906: syn. de Malakichthys D. 1883.

FAMILLE DES MORONIDAE

(Bar, Loup, Loubine ; White-bass)

Chirus (Steller) Cuvier 1829.

Chrysoperca FOWLER 1907.

Dicentrarchus GILL 1860.

Labrax KLEIN 1775 : non valide, syn. de Chirus (S.) C. 1829.

Labrax (K.) CUVIER 1829: syn. de Dicentrarchus G. 1860.

Lepibema RAFINESQUE 1820.

Morone Mrtchill 1814.

Percalates Ramsay et OgIlby 1887.

Roccus MItchILL 1814.

\section{FAMILLE DES LATIDAE}

(Perche-du-Nil ; Ketschr)

Cnidon Müller et Troschel 1849 : syn. de Psammoperca R. 1844.

Hypopterus GILL 1861.

Lates CUVIER et VALENCIENNES 1828.

Luciolates BOULENGER 1914.

Psammoperca RICHARDSON 1844.

Pseudolates Alleyne et MACleay 1877: syn. de Lates C. et V. 1828.

\section{FAM. DES CENTROPOMIDAE} (Robalos)

Blandowskiella IREDALE et WHITLEY 1932.

Centrarchops FOWLER 1924.

Centropomus DE LACÉPÈDE 1803.

Denarusia Whitley 1950.

Gymnochanda FRASER-BRUNNER 1955.

Gymnochanda BOESEMAN 1957.

Konopickia WHITLEY 1937.

Macrocephalus BROWNE 1789: non valide.
Macrocephalus (B.) BLEEKER 1876: syn. de Centropomus L. 1803. Oxylabrax BLEEKER 1876: syn. de Centropomus L. 1803.

Platycephalus RIBEIRO 1902: préemployé, syn. de Centropomus L. 1803.

\section{FAMILLE DES KUHLIIDAE}

(Moutain-bass, Sesele)

Boulengerina FowLER 1904 : pré-employé, syn. de Moronopsis G. 1863.

Dules

Kuhlia G. 1861.

: syn. de

Edelia DE CASTELnaU 1873: syn. de Nannoperca G. 1862.

Herops De VIS 1885: syn. de Kuhlia G. 1861.

Kuhlia GILL 1861.

Microperca DE CASTELNAU 1873: syn. de Nannoperca G. 1862.

Moronopsis GILL 1863.

Nannoperca GüNTHER 1862.

Paradules BleEker 1863: syn. de Kuhlia G. 1861.

Paradules KLUNZINGer 1872: syn. de Nannoperca G. 1862.

Parakuhlia Pellegrin 1913.

Platysoma (LIENARD) SCUDder 1882 : syn. de Moronopsis G. 1863.

Platysome (L.) ScUDDER 1832: syn. de Moronopsis G. 1863.

Safole JoRDan 1912: syn. de Moronopsis GILL 1863.

\section{FAMILLE DES ELASSOMIDAE}

(Dwarf sun-fishes)

Elassoma JORDAN 1877.

Elassosoma JORDAN 1878: errat. pro Elassoma J. 1877.

FAM. DES ACANTHOCLINIDAE Acanthoclinus JENYNS 1842. Acanthoplesiops REgAN 1912.

Ernogrammoides CHENG et LIANG 1948. 
Taumakoides WHITLEY 1955: s./g. d'Acanthoclinus J. 1842.

FAM. DES PSEUDOPLESIOPIDAE Pseudoplesiops BLEEKER 1858.

FAM. DES PSEUDOCHROMIDAE (= Pseudogrammidae)

Acanthagonia OGILBY 1918.

Aporops Schultz 1943.

Assiculus Richardson 1846: syn. de Pseudochromis R. 1835.

Bartschina Fowler 1931: s./g. de Dampieria C. 1875.

Chlidichthys SMITH 1954.

Cichlops Müller et Troschel 1858: syn. de Dampieria C. 1875.

Cypho Myers 1940: syn. de Pseudochromis R. 1835.

Dampieria de Castelnau 1875.

Devisina Fowler 1931: s./g. de Pseudochromis R. 1835.

Gramma POEY 1868.

Klunzingerina FOWLER 1931: s/g. de Psendochromis R. 1835.

Labracinus (SCHLEGEL) BLEEKER 1876: syn. de Dampieria C. 1875.

Labristoma SwaInson 1839: syn. de Pseudochromis R. 1835.

Leptochromis BLEEKER 1876.

Loxopseudochromis FOWLER 1933.

Nematochromis WEBER 1913.

Nesiotes De VIs 1884 : syn. de Pseudochromis R. 1835.

Ogilbyina FOWLER 1931: s./g. de Dampieria C. 1875.

Onar De VIs 1885: syn. de Pseudochromis R. 1835.

Opsipseudochromis FOWLER 1933. Pseudochromichthys SCHMIDT 1931.

Pseudochromis RüPPELL 1835.

Pseudogramma BLEEKER 1875.

Suttonia SмIтH 1953.

Warmizichthys SМITH 1954.
FAMILLE DES PLESIOPIDAE

Assessor Whitley 1935.

Barrosia SMITH 1952.

Belonopterygion Mc CULLOCH 1915.

Bleekeria DE CASTELNAU 1873: préemployé, syn. de Paraplesiops B. 1875 .

Calloplesiops FOWLER et BEAN 1930.

Cirrhiptera (KuHL et VAN Hasselt) BLEEKER 1876: non valide, syn. de Plesiops (C.) O. 1817.

Paraplesiops BLEEKER 1875.

Pharopteryx RüPpell 1828: syn. de Plesiops (C.) O. 1817.

Plesiops (CUVIER) OKEN 1817.

Ruppelia DE CASTELNAU 1873: préemployé, syn. de Paraplesiops B. 1875 .

Tosana SMITH et POPE 1906.

Trachinops GüNTHER 1861.

\section{FAM. DES CENTRARCHIDAE}

$(=$ Eucentarchidae $=$ Micropteridae $)$

(Perche-soleil ; Boers, Black-bass,

Sun-fish, Pumpkin-seeds)

Acantharchus GILL 1864.

Allotis Ortenburger et Hubbs 1927.

Ambloplites RAfINESQUe 1820.

Aplesion RAFINESQUe 1820: syn. de

Micropterus L. 1803.

Aplites RAFINESQUe 1820: syn. de

Micropterus L. 1803.

Apomotis Rafinesque 1819.

Archoplites GILL 1861.

Bartramiolus FOWLER 1945.

Bryttus CuVIER et VALENCIENNES 1831 : syn. d'Apomotis R. 1819. Calliurus RAFINESQUE 1819: syn. de Micropterus L. 1803.

Centarachus CUVIER 1829: serait syn. d'Ambloplites R. 1820.

Centrarchus JORDAN et EvERMANN 1864. : syn. d'Eucentrarchus G.

Chaenobrythus GILL 1864 : errat. pro Chaenobryttus G. 1864. 
Chaenobryttus GILL 1864.

Copelandia JoRDAN 1877 : syn. d'Enneacanthus G. 1864.

Dioplites RAFINESQUE 1820: syn. de Micropterus L. 1803.

Enneacanthus GILL 1864.

Erichaeta JoRDAN 1877: syn. d'Helioperca J. 1877.

Eucentrarchus GILL 1864.

Eupomotis GILL et JoRdAN 1877.

Glossoplites JoRDAN 1876: syn. de Chaenobryttus G. 1864.

Gristes Cuvier 1829: syn. de Micropterus L. 1803.

Grystes : vide Gristes C. 1829.

Helioperca JORDAN 1877.

Hemioplites COPE 1867: syn. d'Enneacanthus G. 1864.

Huro CuVier et Valenciennes 1828.

Hyperistius GILL 1864.

Ichthelis RAFINESQUE 1820: syn. de Lepomis R. 1819.

Lepidopomus : emend. pro Lepomis R. 1819.

Lepiopomus pro Lepomis R. 1819.

Lepemis RAFINESQUE 1819.

Mesogonistius GILL 1864.

Micropterus DE LACÉPÈDE 1802.

Nemocampsis RAFINESQUE 1820 : syn. de Micropterus L. 1803

Pomotis RAFINESQUe 1819: syn. de Eupomotis G. et J. 1877.

Pomotis Cuvier 1829: syn. de Eupomotis G. et J. 1877 .

Pomoxis RAfINESQUe 1818.

Sclerotis ORTENBURGer et HubBs 1927.

Telipomis RAFINESQUE 1820: syn. d'Apomotis R. 1819.

Xenotis Jordan 1877.

Xystroplites JoRDAN 1877: s./g. d'Eupomotis G. et J. 1877.

\section{FAMILLE DES AMBASSIIDAE}

Acanthoperca DE CASTELnaU 1878: syn. d'Ambassis (C.) C. et V. 1828.
Ambassis (COMmerson) Cuvier et VALENCIENNES 1828.

Austrochanda WhITLEY 1935.

Bogoda BLEEKER 1853: syn. de Chanda H. 1822.

Chanda Hamilton-Buchanan 1822. Hamiltonia SwaInson 1839: syn. de Chanda H. 1822.

Negambassis WhITLEY 1935.

Parambassis BLEEKER 1874.

Priopidichthys WhITLEY 1935.

Priops (KuHL et Van Hasselt) CuVIER et VALENCIENNES 1830.

Pseudambassis BLEEKER 1876.

Pseudoambassis de CASTelnau 1878: syn. de Austrochanda W. 1935.

Synechopterus NORMAN 1935.

Tetracentrum MACLEAY 1883: préemployé, syn. de Negambassis W. 1935.

Velambassis WHITLEY 1935.

Whitleyia : syn. d'Ambassis (C.) C. et V. 1828.

Whitleyina FOWLER et BEAN 1930: s./g. d'Ambassis (C.) C. et V. 1828.

Xenambassis Schultz 1945.

FAM. DES ACROPOMATIDAE Acropoma Temminck et Schlegel 1843.

Brephostoma AlCOCK 1889.

Oxyodon BRAUER 1906.

Parascombrops Alcock 1890: syn. d'Acropoma T. et S. 1843.

FAMILLE DES RHEGMATIDAE Rhegma GILBERT 1900.

FAMILLE DES PRIACANTHIDAE (Big-eyes)

Abuhamrur FORSKAL 1771: non valide.

Bogota Blyth 1860: syn. de Priacanthus C. 1817.

Boops Gronow 1854: syn. de Priacanthus C. 1817. 
Priacanthus CUVIER 1817.

Pristigenys AgASSIZ 1835.

Pseudopriacanthus BLEEKER 1869: syn. de Pristigenys A. 1835.

\section{FAMILLE DES PEMPHERIDAE (Catatufas)}

Catalufa SNYDER 1911.

Leptobrama STEINDACHNER 1878.

Liopempheris OGILBY 1913.

Neopempheris Macleay 1881: syn. de Leptobrama S. 1878.

Parapempheris BONDE 1924.

Parapriacanthus STEINDACHNER 1870.

Pempherichthys KLUNZINGER 1871: syn. de Parapriacanthus S. 1870.

Pempheris CUVIER 1829.

Priacanthopsis FOWLER 1906.

\section{FAMILLE DES APOGONIDAE}

$(=$ Amiidae $=$ Cheilodipteridae $)$

Acanthapogon FowLER 1938.

Adenapogon Mc Culloch 1921. Amia Gronow 1763: non valide. Amia Gronow 1854 : syn. d'Apogon L. 1802.

Amioides Smith et RAdCliffe 1912. Apogon de Lacépède 1802. Apogonichthyoides SMITH 1949. Apogonichthys BLEEKER 1854. Apogonoides BLEEKER 1849. Archamia GILL 1863. Aspiscis WhItLey 1930. Aspro (COMMERSON) DE LACÉPÈdE 1803: non valide.

Astrapogon FoWLeR 1907.

Bathysphyraenops PARR 1933.

Brephamia JORDAN 1922.

Brinkmannella PARR 1933.

Cheilodipterops SchULTZ 1940.

Cheilodipterus DE LACÉPÈDE 1802. Chilodipterus: vide Cheilodipterus
Clodipetrus RAFINESQUE 1815: syn. de Cheilodipterus L. 1802.

Desmoamia Fowler et BEAN 1930 : s./g. de Cheilodipterus L. 1802.

Dinolestes KLUNZINGER 1872.

Dipterodon DE LACÉPÈDE 1803 : syn. d'Apogon L. 1802.

Epigonus RAFINESQUE 1810.

Erythrobussothen PARR 1933.

Foa Jordan et EVERMANn 1905.

Fodifoa WhItLey 1936.

Fowleria JoRdAn et Evermann 1903.

Glossamia GILL 1863.

Gronovichthys.

Gulliveria DE CASTELnAU 1878: syn. de Glossamia G. 1863.

Gymnapogon REGAN 1905.

Hynnodus GILBERT 1905.

Ioamia Fowlen et BeAN 1930 : s./g. d'Archamia G. 1863.

Jadamga SchULtZ 1940.

Kurandapogon WHITLEY 1939.

Lachneria Sмiтн 1954 : syn. de Pseudamiops S. 1954.

Lanioperca GüNTHER 1872: syn. de Dinolestes K. 1872.

Lepidamia GILL 1863.

Lovamia WhITLEY 1930.

Maccullochina JORDAN 1923.

Macrolepis RAFINESQUE 1815: syn. d'Apogon L. 1802.

Melanostoma STEINDACHNER et DöDERLEIN 1883 : pré-employé, syn. de Synagrops G. 1887.

Microichthys RüPPELL 1852.

Microperca DE CASTELnAU: pré-employé, syn. de Percamia B. 1876.

Mimocubiceps FOWLER 1944.

Mionorus KREFFT 1867.

Monoprion Poey 1860: syn. d'Apogon L. 1802.

Monosira POEY 1881: syn. d'Apogon L. 1802.

Nannapogon FowLER 1938.

Neamia SMith et RadCliffe 1912. 
Nectamia JoRdan 1917.

Neoscopelus: syn. d'Adenapogon MC. 1921.

Neosphyraena DE CASTELNAU 1872: syn. de Dinolestes K. 1872.

Ostorhinchus DE LACÉPÈDE 1803.

Papillapogon Sмттн 1946.

Parahynnodus BARNARD 1927.

Paramia BleEker 1863: syn. de Cheilodipterus L. 1802.

Percamia BleEker 1876.

Pomatoichthys Giglioli 1880: syn. d'Epigonus R. 1810.

Pomatonus (RISso) Cuvier et VAlenCIENNES 1828: syn. d'Epigonus R. 1810 .

Pristiapogon KLUNZINGER 1870: syn. d'Apogon L. 1802.

Pseudamia BleEker 1865.

Pseudamiatus Whitley 1954.

Pseudamiops SмITH 1954.

Pterapogon Koumans 1933.

Rhabdamia WEBER 1909.

Sceptrias Jordan et JORDAN 1923.

Scombrolabrax ROULE 1922.

Scopelapogon WHITLEY 1933: s./g. d'Adenapogon MC. 1921.

Siphamia WEBER 1909.

Sphaeramia Fowler et BEAN 1930 : s./g. d'Apogon L. 1802.

Synagrops GüNTHER 1887.

Telescops Bleeker 1876: syn. d'Epigonus R. 1810.

Vincentia DE CASTelnau 1872: syn. d'Apogon L. 1802.

Xystramia JoRdAN 1917.

Yarica WhITLEY 1930.

Zoramia JORDAN 1917.

FAMILLE DES SCOMBROPIDAE Amiichthys POEY 1886.

Apogonops OGILby 1896.

Galeagra Heller et SNODgrass 1903: syn. de Sphyraenops P. 1860.

Hypoclydonia GoODE et BEAN 1895. Latebrus POEY 1860.

Neoscombrops GILCHRIST 1922.
Parasphyraenops BEAN 1912.

Rhectogramma NORMAN : syn. de Sphyraenops P. 1860.

Scombrops Temminck et Schlegel 1845.

Sphyraenops POEY 1860.

Telescopias JORDAN et SNYDER 1901: syn. de Scombrops T. et S. 1845.

FAMILLE DES PERCILIIDAE

Percilia GIRARD 1854.

\section{FAM. DES PERCICHTHYIDAE}

Deuteropterus GILL 1861.

Percichthys GIRARD 1854.

Percosoma GILL 1861.

FAM. DES ETHEOSTOMIDAE

(= Etheostomatidae)

(Darters, Crawl-a-bottom)

Alvarius GIRARD 1859.

Alvordius GIRARD 1859.

Ammocrypta JoRDAN 1877.

Anemoces Jordan 1929.

Arlina GIRARD 1859: syn. de Boleosoma D. 1842.

Asproperca HeCKel 1860: syn. de Percina H. 1842.

Astatichthys VAILLANT 1873: syn. d'Oligocephalus G. 1859.

Austroperca HuBBs 1936.

Boleichthys GIRARD 1859.

Boleosoma DEKAY 1842.

Catonotus AgAssiz 1854.

Claricola JoRdÁn et EVERMANN 1896.

Copelandellus Jordan et EVERMANN 1896.

Cottogaster Putnam 1863.

Crystallaria JoRdan et G I L B E R T 1885.

Diplesion RAFINESQUe 1820: syn. d'Etheostoma R. 1819.

Doration JoRDAN 1929.

Ericosma JoRDAN et COPELAND 1877: s./g. d'Hadropterus A. 1854. 
Estrella GIRARD 1859 : syn. de Boleosoma D. 1842.

Etheostoma RAFINESQUE 1819.

Etheostoma JORDAN et EVERMANN 1896: syn. de Poecilichthys A. 1854.

Ethestoma Agassiz 1854: errat. pro Etheostoma R. 1819.

Hadropterus Agassiz 1854.

Hololepis (Agassiz) Putnam 1863.

Hyostoma AgAssiz 1854: syn. d'Etheostoma R. 1819.

Hypohomus COPE 1870.

Imostoma JORDAN 1877.

Ioa JORDAN et BRAYTON 1878.

Litocara BAILEY 1948: s./g. de Poecilichthys A. 1854.

Microperca PUTNAM 1863.

Nanostoma (PUTNAM) JORDAN 1877: pré-employé, syn. de Rhothoeca J. 1885.

Nivicola JORDAN 1896 : pré-employé, syn. de Niviperca W. 1953.

Niviperca WHITLEY 1953.

Nothonotus (Agassiz) PUtNam 1863: s./g. d'Etheostoma R. 1819.

Oligocephalus GIRARD 1859.

Percina Haldeman 1842.

Pileoma Dekay 1842: syn. de Percina H. 1842.

Plesioperca VAILlant 1873: syn. d'Hadropterus A. 1854.

Pleurolepis (Agassiz) Putnam 1863 : pré-employé, syn. de Vigil J. 1919.

Poecilichthys Agassiz 1854.

Poecilosoma Agassiz 1850 : pré-employé, syn. de Poecilichthys A. 1854.

Psychromaster JoRdan et EVERMANN 1896.

Rafinesquiellus JORDAN et EVERMANN 1896.

Rheocrypta JoRDAN 1877: s./g. de Cottogaster P. 1863.

Rhothoeca JORDAN 1885.

Serraria GILBERT 1884.

Swainia JoRDAN et EVERMANN 1896. Torrentaria J O R D A N et EvermanN
1896: pré-employé, syn. d'Aus. troperca H. 1936.

Ulocentra JORDAN 1878.

Vaillantia JORDAN 1878.

Vigil JORDAN 1919.

\section{FAMILLE DES PERCIDAE}

(Perches, Sandre, Apron, Grémille ; Perch)

Acerina Güldenstadt 1774.

Acerina Cuvier 1817: syn. d'Acerina G. 1774.

Acerina Cuvier 1820: syn. d'Acerina G. 1774.

Aplocentrus RAFINESQUE 1819: non valide.

Asper SchäFER 1761.

Asperulus SchäFER 1761: syn. de Zingel (C.) O. 1817.

Asperulus KLEIN 1775: non valide. Asperulus (K.) GILL 1861: syn. de Zingel (C.) O. 1817.

Aspro Cuvier et Valenciennes 1828 : syn. de Zingel (C.) O. 1817.

Belophlox FOWLER 1947.

Bidyanus WHITLEY 1943.

Cephimmus RAFINESQUE 1815: syn. de Acerina G. 1774.

Cernua SchäFER 1876: syn. d'Acerina G. 1774.

Cernua Fleming 1828: syn. d'Acerina G. 1774.

Cingla StaRK 1828: syn. de Zingel (C.) O. 1817.

Cynoperca GILl et Jordan 1877.

Epitrachys Schulze 1889: syn. de Perca L. 1758.

Gremilla Gistel 1848: syn. d'Acerina $G .1774$.

Gymnocephalus BLocH 1793: syn. d'Acerina G. 1774.

Leptoperca GILL 1861.

Leucops RAFINESQUE 1819: non valide.

Lucioperca CuVIER et VALENCIENNES 1828: syn. de Sander (C.) O. 1817.

Mimoperca GILL et JoRDAN 1877 : syn. de Schilus K. 1832. 
Perca LinNé 1758.

Percarina Nordmann 1840.

Percis KLeIN 1775 : non valide, syn. d'Acerina G. 1774.

Peroptera GISTEL 1848: non valide.

Pogostoma RAFINESQUE 1818: non valide.

Pomacampsis RAFINESQUE 1820 : non valide.

Richia COKER 1927.

Richiella COKER 1927.

Romanichthys DIMITRESCU 1958.

Sandat Cloquet 1827: syn. de Sander (C.) O. 1817.

Sandat BORY DE ST-VINCENT 1828: syn. de Sander (C.) O. 1817.

Sander (CUVIER) OKEN 1817.

Sandrus Stark 1828 : syn. de Sander (C.) O. 1817.

Schilus KRYNICKI 1832.

Schraitzer ScHäFER 1761 : non valide, syn. de Leptoperca G. 1861.

Stizostedion RAFINESQUE 1820.

Stizostethium: vide Stizostedion R. 1820.

Villora HuBBs et CANNON 1935.

Zingel (CUVIER) OKEN 1817.

FAM. DES MALACANTHIDAE

Malacanthus CUVIER 1829.

Oceanops Jordan et Seale 1906.

FAMILLE DES LATILIDAE

(= Branchiostegidae) (Tile Fish)

Branchiostegus RAFINESQUE 1815: syn. de Latilus C. et V. 1830.

Caulolatilus GILL 1862.

Coryphaenoides DE LACÉPÈDE 1802 : pré-employé, syn. de Latilus C. et V. 1830.

Dekaya COOPER 1863: pré-employé, syn. de Caulolatilus G. 1862.

Dikellorhynchus SMITH 1956.

Hoplolatilus GÜNTHER 1887.

Latilus CUVIER et VALENCIENNES 1830.
Lopholatilus GoODE et BEAN 1879. Prolatilus GILL 1865.

\section{FAMILLE DES OTOLITHIDAE}

(Weak-fishes, Queen-fishes)

Ancylodon (CUVIER) OKEN 1817: pré-employé, syn. de Macrodon S. 1822 .

Apseudobranchus GILL 1862: syn. de Cestreus Gronow 1854.

Archoscion GILL 1862.

Atractoscion GILL 1862.

Bahaba HerRe 1935: s./g. d'Otolithes (C.) O. 1817.

Buccone JoRDAN et EVERMANN 1896.

Cestreus Gronow 1854.

Cynoscion Gill 1861: syn de Cestreus G. 1854.

Isopisthus GILL 1862.

Macrodon SCHINZ 1822.

Nebris CuVie R et Valenciennes 1830.

Nomalus Gistel 1848: syn. de Macrodon S. 1822.

Otolithes (CUVIER) OKEN 1817.

Otolithoides FOWLER 1933.

Otolithus CuVIER: vide Otolithes (C.) O. 1817.

Pama Fowler 1933.

Paraplesichthys (K A U P) BLEEKER 1876: non valide, syn. d'Isopisthus G. 1862.

Pterotolithus FowLER 1933: s./g. d'Otolithes (C.) O. 1817.

Sagenichthys BERG 1895: syn. de Macrodon S. 1822.

Seriphus AYRES 1854.

Symphysoglyphus RIBEIRO 1913.

FAMILLE DES SILLAGINIDAE

Iosillago Macleay 1878: syn. de Sillago C. 1817.

Neosillago de Castelnau 1875.

Sillaginichthys BLEEKER 1876.

Sillaginodes GILL 1861.

Sillaginopodys FOWLER 1933: s./g. de Sillago C. 1817. 
Sillaginopsis GILL 1861.

Sillago CUVIER 1817.

\section{FAMILLE DES POMATOMIDAE}

(Tassergal ou Poisson-bleu ; Blue-Fishes)

Chromis Gronow 1854 : syn. de Pomatomus L. 1803.

Gonenion RAFINESQUe 1810 : syn. de Pomatomus L. 1803.

Lopharis RAFINESQUE 1810: syn. de Pomatomus L. 1803.

Pomatomus DE LACÉPÈde 1803.

Saltatrix CATESBY 1771: non valide, syn. de Pomatomus L. 1803.

Sparactodon DE ROCHEBRUNE 1880: syn. de Pomatomus L. 1803.

Sypterus Eichewald 1841: syn. de Pomatomus L. 1803.

Temnodon CuvIER 1817: syn. de Pomatomus L. 1803.

FAM. DES RACHYCENTRIDAE (Sergent ; Sergeant-fish)

Elacate CUVIER et VALENCIENNES 1831: syn. de Rachycentron K. 1826 .

Meladerma SwaInson 1839: syn. de Rachycentron K. 1826.

Rachycentron KAUP 1826.

Rachycentrum: vide Rachycentron K. 1826 .

Spinax (COMmerson) CUVIER et VAI.ENCIENNES 1831: syn. de $\boldsymbol{R a}$ chycentron K. 1826.

\section{FAMILLE DES LACTARIIDAE}

Lactarius CUVIER et VALENCIENNES 1833.

Platylepes SwaInson 1839: syn. de Lactarius C. et V. 1833.

FAMILLE DES APOLECTIDAE

Apolectus CuVier et Valenciennes 1831.

Parastromateus BLEEKER 1865: syn. d'Apolectus C. et V, 1831.
FAMILLE DES CARANGIDAE

(Chinchards, Fanfres, Liches, Sérioles ; Cavallas)

Absalom WhItLey 1937.

Acanthinion DE LACÉPÈDE 1803 : syn. de Trachinotus L. 1802.

Alectis RAFINESQUE 1815.

Alepes Swainson 1839.

Argyreiosus DE LACÉPÈDE 1803 : syn. de Selene L. 1803.

Atropus (CUVIER) OKEN 1817 : s./g. de Carangoides B. 1851.

Atule Jordan et Jordan 1923 : s./g. de Caranx L. 1802.

Atule Nichols 1942: syn. d'Atule J. et J. 1923.

Baillonus RAFINESQUE 1815: syn. de Caesiomorus L. 1802.

Bassetina WHITLEY 1941.

Blepharichthys GILL 1861: syn. d'Alectis R. 1815.

Blepharis CUVIER 1817 : pré-employé, syn. d'Alectis R. 1815.

Bothrolaemus HolbrooK 1855: syn. de Trachinotus L. 1802.

Branchialepis FOWLER 1938: s./g. d'Alepes S. 1839.

Buphthalmus SмIтH 1959: s./g. de Seriola C. 1816.

Caesiomorus DE LACÉPÈDE 1802.

Campogramma REgAN 1903: syn. de Caesiomorus L. 1802.

Carangichthys BLEEKER 1852: syn. de $\operatorname{Caranx}$ L. 1802.

Carangoides BLEEKER 1851.

Carangops GILL 1862: syn. d'Alepes S. 1839.

Carangulus JORDAN et EVERMANN 1927: s./g. de Caranx L. 1802.

Carangus Agassiz 1845: syn. de Ca$\operatorname{ran} x$ L. 1802.

Carangus GRIFFITH 1834: syn. de Caranx L. 1802.

Carangus Girard 1859: syn. de Ca$\operatorname{ranx}$ L. 1802.

Caranx DE LACÉPÈdE 1802.

Centronotus DE LACÉPÈDE 1802 : préemployé, syn. de Naucrates R. 1810 . 
Chloroscombrus GIRARD 1859.

Chorinemus CUVIER et VALENCIENNES 1832: syn. de Scomberoides L. 1802.

Citula Cuvier 1817 : s./g. de Carangoides B. 1851.

\section{Citularia.}

Decapterus BLEEKER 1851.

Decapterus POEY 1851: syn. d'Elagatis B. 1840 .

Doliodon GIRARD 1859 : syn. de Trachinotus L. 1802.

Elagatis BENNETT 1840.

Elaphotoxon FOWLER 1905.

Eleria Jordan et Seale 1905.

Eustomatodus GiLL 1862: syn. de Decapterus B. 1851.

Evepigymnus GILL 1862.

Ferdauia Jordan, EVERMANN et TANAKA 1927.

Fonnio Mac Culloch 1929.

Gallichthys Cuvier et VALENCIENNES 1833: syn. d'Alectis R. 1815.

Gallus DE LACÉPÈDE 1803 : syn. d'Alectis R. 1815.

Glaucus KLEIN 1775 : non valide, syn. de Caesiomorus L. 1802.

Glaucus (K.) BLEEKER 1863: syn. de Caesiomorus L. 1802.

Glaucus (K.) Fowler 1906: syn. de Caesiomorus L. 1802.

Glaucus (K.) JoRdAN et HubBs 1917 : syn. de Caesiomorus L. 1802.

Gnathanodon BLEEKER 1951: syn. de Gnathodon B. 1851.

Gnathodon BLEEKER 1851.

Guaperva (PluMIER) DE LACÉPÈDE 1803 : non valide, syn. de Selene L. 1803 .

Gymnepignathus GILL 1862.

Halatractus GILl 1862: syn. de Seriola C. 1816.

Hemicaranx BLEEKER 1862: syn. d'Alepes S. 1839.

Hildebrandella Nichols 1950.

Hynnis CUVIER et VALENCIENNES 1833.

Hypacantha RAFINESQUE 1810: syn. de Caesiomorus L. 1802.

Hypacantus: vide Hypacantha R. 1810.
Hypocaranx KLUNZINGER 1884: syn. de Gnathodon B. 1851.

Hypodis RAFINESQue 1810: syn. de Caesiomorus L. 1802.

Irex VALENCIENNES 1862 : syn. d'Elagatis $B .1840$.

Juvenella WHITLEY 1950.

Leioglossus BLEEKER 1851.

Lepidomegas TноміNот 1880: syn. de Seriola C. 1816.

Leptaspis BLEEKER 1852: syn. de Selaroides B. 1851.

Leptoligoplites FOWLER 1944 : s./g. d'Oligoplites G. 1863.

Leucoglossa JORDAN, EVERMANN et TANAKA 1927.

Lichia CuVIER 1817 : syn. de Caesiomorus L. 1802.

Longirostrum WAKIYA 1923: syn. d'Uraspis B. 1855.

Megalaspis BLEEKER 1851.

Melbanella WHITLEY 1937.

Micropteryx Agassiz 1829: syn. de Seriola C. 1816.

Micropus KNER 1868: pré-employé, syn. d'Orqueta J. 1919.

Nauclerus Cuvier et VAlenciennes 1833: syn. de Naucrates R. 1810 .

Naucrates RAFINESQUE 1810.

Naucratopsis GILL 1862.

Oligoplites GILL 1863.

Olistus Cuvier 1829: s./g. de Carangoides $B .1851$.

Orcynus RAFINESQUE 1815 : pré-employé, syn. de Scomberoides L. 1802.

Orqueta JORDAN 1919.

Pampanoa FoWLer 1906.

Pantolabus WhItLey 1931.

Paratractus GILL 1862.

Parona Berg 1895.

Paropsis JenYNS 1842: pré-employé, syn. de Parona B. 1895.

Pelamis (Plumier) DE LAcépède 1802: non valide, syn. d'Oligoplites G. 1863.

Platysomus SwaInson 1839: syn. de Vomer C. 1817. 
Platyuraspis FOWLER 1938: s./g. de Uraspis B. 1855.

Pompilus Minding 1832: syn. de Naucrates R. 1810.

Porthmeus Cuvier et Valenciennes 1833: syn. de Caesiomorus L. 1802.

Pseudocaranx BLEEKER.

Rastrum FOWLER 1964.

Regificola WHITLEY 1931.

Rhaphiolepis FOWLER 1905.

Rhomboida BROWNE 1789: non valide, syn. de Vomer C. 1817.

Saurus BROWNE 1789: non valide.

Scomberoides DE LACÉPÈdE 1802.

Scyris CuvIER 1828: syn. d'Alectis R. 1815 .

Selar Bleeker 1851: s./g. de Caranx L. 1802 .

Selaroides BLEEKER 1851.

Selene DE LACÉPÈdE 1803.

Selenia BONAPARTE 1845: pré-employé, syn. d'Uraspis B. 1855.

Seriola CUVIER 1816.

Seriolichthys BLEEKER 1854 : syn. d'Elagatis B. 1840.

Seriolina WAKIYA 1923.

Suarenus FURNSTEIN 1958.

Thynnus GRONOW 1854: pré-employé, syn. de Scomberoides L. 1802.

Trachinotus DE LACÉPÈDE 1802.

Trachurops GILL 1862 : syn. de Selar B. 1851 .

Trachurus (PLUMIER) DE LACÉPÈDE 1802: non valide, syn. de Trachurus R. 1810.

Trachurus RAFINESQUE 1810.

Trachurus GRONOW 1854: syn. de Megalaspis B. 1851.

Trachynotus: vide Trachinotus L. 1802.

Tricopterus RAFINESQUE 1810: syn. de $\operatorname{Caranx}$ L. 1802.

Turrum WhITLEY 1932.

Ulua JORDAN et SNYDER 1908.

Uraspis BLEEKER 1855.

Usa Whitley 1927 : s./g. de Caranx L. 1802.

Usacaranx WhITLEY 1931,
Vexillicaranx FOWLER 1905.

Vomer CUVIER 1817.

Vomeropsis HeCKel 1843.

Xurel Jordan et Evermann 1927.

Xystrophorus RICHARDSON 1844 : larve de Naucrates R. 1810.

Zalocys JoRDAN et Mc GREgor 1898. Zamora WhITLEY 1931 : pré-employé, syn, de Bassetina W. 1941.

Zonichthys SWAINSON 1839: s./g. de Seriola C. 1816.

FAMILLE DES NEMATISTIIDAE

(Poissons-paons ; Peacock-fishes)

Nematistius GILL 1862.

Seriolophus GUICHENOT 1867: syn. de Nematistius G. 1862.

\section{FAMILLE DES MENIDAE}

Mene de LACÉPÈdE 1803.

Meneus RAFINESQUE 1815: syn. de Mene L. 1803.

FAM. DES EMMELICHTHYIDAE (= Erythrichthyidae)

Boxaodon GUICHENOT 1848.

Emmelichthyops SchULTZ 1945.

Emmelichthys RICHARDSON 1844. Erythrichthys TemMincK et Schlegel 1845: pré-employé, syn. d'Erythrocles J. 1919.

Erythrocles JORDAN 1919.

Inermia PoEY 1860: syn. d'Emmelichthys R. 1844.

Plagiogeneion ForBes 1890.

Schultzea Woods 1958.

FAMILLE DES DIPLOPRIONIDAE

Diploprion (KuHL et Van Hasselt) CUVIER et VALENCIENNES 1828.

FAMILLE DES LOBOTIDAE (Flashers)

Coius Hamilton-Buchanan 1822. 
Datnioides BLEeKer 1852: syn. de Coius H. 1822.

Lobotes CuVIER 1829.

Verrugato JORDAN 1923.

\section{FAM. DES GLAUCOSOMIDAE}

Brachyglaucosoma FOWLER 1934: s./g. de Glaucosoma T. et S. 1843 .

Breviperca de CASTELnaU 1873: larve de Glaucosoma T. et S. 1843.

Glaucosoma TEMMINCK et SCHLEgel 1843.

Reganichthys OgILby 1915: syn. de Glaucosoma T. et S. 1843.

\section{FAMILLE DES ARRIPIDAE}

Arripis Jenyns 1842.

Homodon BRISOUT DE BARNEVILle 1847 : syn. d'Arripis J. 1842.

\section{FAM. DES XENICHTHYIDAE} (serait syn. de Pomadasyidae fide

$$
\text { Regan) }
$$

Cheiroxenichthys FOWLER 1930: s./g. de Xenichthys G. 1863.

Xenichthys GILL 1863.

Xenistius Jordan et Gilbert 1882.

Xenocys Jordan et Bollman 1889.

FAMILLE DES HOPLOPAGRIDAE Hoplopagrus GILL 1861.

\section{FAMILLE DES LUTJANIDAE (Snappers)}

Aetiasis BARNARD 1937.

Anthea CATEsBy 1771: non valide. Aprion Cuvier et Valenciennes 1830.

Apsilus Cuvier et Valenciennes 1830.

Arnillo Jordan, Evermann et TANAKA 1927.

Bennettia FOWLER 1904.
Bowersia Jordan et Evermann 1903 : syn. de Pristipomoides B. 1852.

Caesio DE LACÉPÈdE 1802.

Caesioscorpis WHITLEY 1945.

Chaetopterus Temminck et Schlegel 1844 : pré-employé, syn. de Pristipomoides B. 1852.

Clupeolabrus Nichols 1923.

Conodon Cuvier et VAlenciennes 1830.

Diacope Cuvier 1815: pré-employé, syn. de Genyoroge C. 1849.

Elastoma SWAINSON 1839 : syn. d'Etelis C. et V. 1828.

Etelides JoRDAN et THOMPSON 1905.

Etelinus JORDAN et THOMPSON 1911.

Etelis Cuvier et VAlenciennes 1828.

Evoplites GILL 1862.

Genyoroge CANTOR 1849.

Glabrilutjanus FOWLER 1931: s./g. de Lutjanus B. 1790.

Hesperanthias Lowe 1843: syn. d'Etelis C. et V. 1828.

Hobar ForsKal 1775: non valide.

Hypoplites GILL 1862.

Jordanichthys EVERMANN et CLARK 1928.

Loxolutjanus FOWLER 1931: s./g. de Lutjanus B. 1790.

Lunicauda WHITLEY 1947.

Lutianus: vide Lutjanus B. 1790.

Lutjanus BLOCH 1790.

Macolor BLEEKER 1860.

Macrops DUMÉRIL 1856 : pré-employé, syn. d'Etelis C. et V. 1828.

Mesoprion Cuvier et VAlenciennes 1828 : syn. de Lutjanus B. 1790.

Mirolabrichthys HERRE 1927.

Naqua ForsKal 1771: non valide, syn. de Genyoroge C. 1849.

Neomaenis GIRARD 1859.

Neomesoprion dE CASTELnAU 1875 . syn. de Lutjanus B. 1790.

Ocyurus GILL 1862.

Pagrus (Plumier) De LACÉPÈde 1803: non valide, syn. de Neomaenis G. 1859 .

Paradicichthys WhITLEY 1930. 
Parkia Fowler 1904.

Platyinius GILl 1862: syn. de Pristipomoides B. 1852.

Pristipomoides BLEEKER 1852.

Proamblys GILl 1862: syn. de Macolor B. 1860.

Pseudopentaceros BLEEKER 1876.

Rabirubia JORDAN et FESLER 1893.

Raizero JORDAN et FESLER 1893.

Rhomboplites GILL 1862.

Rhomboplitoides FOWLER 1918.

Rooseveltia JORDAN et EVERMANN 1906.

Salpa CATESBY 1771 : non valide, syn. de Neomaenis G. 1859.

Sarda (Plumier) DE LACÉPÈde 1802 : non valide, syn. d'Ocyurus G. 1862.

Sargus (P.) DE LACÉPÈDE 1803: non valide, syn. de Neomaenis G. 1859.

Sciaenus (COMMERSON) DE LACÉPÈDE 1802: non valide.

Sparopsis Kner 1868: syn. d'Aprion C. et V. 1830.

Strophodus Agassiz 1838.

Tropidinius (GILL) POEY 1868.

Turdus CATESBY 1771: non valide.

Ulapiscis WHITLEY 1933.

Ulaula JoRDAN et THOMPSON 1911 : syn. de Pristipomoides B. 1852.

Vegetichthys TANAKA 1918.

\section{FAMILLE DES NEMIPTERIDAE $(=$ Denticidae $)$}

Anemura Fowler 1904: syn. de Synagris (K.) B. 1876.

Euthyopteroma FOWLER 1904.

Gnathodentex BLEEKER 1873.

Gymnocranius KLUNZINGER 1870.

Nemipterus SwaINSON 1839.

Odontoglyphis FOWLER 1904.

Paradentex BLEEKER 1876: syn. de Gymnocranius K. 1870.

Polysteganus KLUNZINGER 1870.

Scaevius WhITLEY 1947.

Sparopsis KNER 1868: syn. de Synagris (K.) B. 1876.
Synagris KLEIN 1775: non valide.

Synagris GüNTHER 1859: syn. de Nemipterus S. 1839.

Synagris (K.) BLEEKER 1876.

\section{FAM. DES LEIOGNATHIDAE}

Argylepes SwaInson 1839: syn. de Leiognathus L. 1803.

Aurigequula FOWLER 1918.

Deveximentum FOWLER 1904: syn. de Leiognathus L. 1803.

Equula Cuvier 1817: syn. de Leiognathus L. 1803.

Equulites FOWLER 1904.

Eubleekeria FOWLER 1904.

Eugeries Jordan et EVERMANN 1927.

Gazza RüPpell 1835.

Halex (COMMERSON) DE LACÉPÈDE 1803 : non valide, syn. de Leiognathus L. 1803.

Leiognathus DE LACÉPÈDE 1803.

Macilentichthys WHITLEY 1932.

Nuchequula WHITLEY 1932.

Parochusus Whitley 1930.

Pentaprion BleEKeR 1850.

Pertica Fowler: pré-employé, syn. de Victor W. 1953.

Secutor Gistel 1848: s./g. de Leiognathus L. 1803.

Victor WhITLEY 1953.

FAMILLE DES VERILIDAE

Verilus POEY 1860.

FAMILLE DES APHAREIDAE

Aphareus Cuvier et VAlenciennes 1830.

Fares Jordan, Evermann et TANAKA 1927 : pré-employé, syn. de $\boldsymbol{H u}$ mefordia W. 1931.

Humefordia WHITLEY 1931: s./g. d'Aphareus C. et V. 1830.

Sacrestinus JORDAN, EVERMANN et TANAKA 1927: s./g. d'Aphareus C. et V. 1830. 
FAMILLE DES POMADASYIDAE $(=$ Haemulidae $=$ Pristipomidae $=$ Pristipomatidae) (Grunts)

Anarmostomus (SCUdDER) PUtNAM 1863 : pré-employé, syn. d'Haemulon C. 1829.

Anisotremus GILL 1860.

Anomalodon BowDICH 1825 : syn. de Pomadasys L. 1803.

Apostata (Heckel) Canestrini 1860 : syn. de Conodon C. et V. 1830.

Bathystoma SCUDDER 1863.

Brachydeuterus GILL 1862.

Brachygenys (SCUDDER) POEY 1868.

Caesio DE LACÉPÈdE 1802.

Cheloniger (PLUMIER) DE LACÉPÈDE 1803 : non valide, syn. de Conodon C. et V. 1830.

Ctenoscolopsis FowLer 1931: s./g. de Scolopsis C. 1815.

Dacymba JoRdan et HubBs 1917.

Diabasis Desmarest 1823: pré-employé, syn. d'Haemulon C. 1829.

Diagramma Cuvier 1815: syn. de Plectorhynchus L. 1802.

Diagramella Pellegrin 1912.

Euelatichthys FOWLER 1904.

Eurumetops MORTON 1887.

Evapristis JORDAN et EVERMANN 1896.

Gaterin ForSKAL 1775: non valide.

Genyatremus GILL 1862.

Genytremus GILL 1862: vide Genyatremus G. 1862.

Ghanan ForsKal 1775: non valide.

Gymnocaesio BLFEKER 1876.

Haemulon CUVIER 1829.

Haemulopsis STEINDACHNER 1869: syn. de Brachydeuterus G. 1862.

Haemylum : vide Haemulon C. 1829.

Hapalogenys RICHARDSON 1844.

Heterodon BLEEKER 1845.

Heterognathodon BLEEKER 1848.

Hyperoglyphe GüNTHER 1859.
Isacia JORDAN et FESLER 1893.

Isaciella JORDAN et FESLER 1893.

Isaciops MILES 1953.

Leitectus SMITH 1952.

Lepidopristis FOWLER 1944: s./g. d'Orthopristis G. 1859.

Lepthaemulon FOWLER et BEAN 1923: s./g. d'Orthopristis G. 1859.

Leptoscolopsis TANAKA 1915.

Liocaesio BLEEKER 1876.

Luzonichthys HERRE 1936.

Lycogenis (KUHL et VAN HASSELT) CUVIER et VALENCIENNES 1830 .

Lythrulon JORDAN et SWAIN 1884.

Mylacrodon REGAN 1903.

Odontonectes GÜNTHER 1859.

Orthopristis GIRARD 1859.

Orthostoechus GILI. 1862.

Otoperca BOULENGER 1916: syn. de Brachydeuterus G. 1862.

Paracaesio BLEEKER 1875.

Paraconodon BLEEKER 1876.

Parapristipoma BLEEKER 1873.

Parascolopsis BOULENGER 1901.

Pinjalo BLEEKER 1845.

Plectorhinchus DE LACÉPÈDE 1802.

Plectorhynchus : vide Plectorhinchus L. 1802.

Pluchus SмIтH 1949.

Pomadasyina FoWLer 1931: s./g. de Pomadasys L. 1803.

Pamodasys DE LACÉPÈdE 1803.

Pristipoma CUVIER 1817: vide Pristipomus (C.) O. 1817.

Pristipomus (CUVIER) OKEN 1817: syn. de Pomadasys L. 1803.

Pristocantharus GILL 1862: syn. d'Orthopristis G. 1859.

Propoma Günther 1880.

Pseudopristipoma SaUVage 1880.

Pterocaesio BleEKer 1876.

Rhencus JORDAN et EVERMANN 1896.

Rhonciscus JORDAN et EVERMANN 1896.

Scolopsides Cuvier 1829: syn. de Scolopsis C. 1815. 
Scolopsis CUVIER 1815.

Spilotichthys FOWLER 1904.

Stenoscolopsis Fowler: syn. de Lycogenis (K. et V. H.) C. et V. 1830.

\section{FAMILLE DES THERAPONIDAE}

Amniataba WHITLEY 1943.

Amphitherapon WhITLEY 1943.

Autisthes DE VIS 1885: syn. de Therapon C. 1817.

Datnia Cuvier et Valenciennes.

Datniodes (BRISOUT DE BARNEVILLE) CANESTRINI 1860: syn. de Therapon C. 1817.

Djabub ForSKaL 1775: non valide, syn. de Therapon C. 1817.

Eutherapon FOWLER 1904.

Helotes CuVIER 1829.

Hephaestus DE VIS 1885.

Leiopotherapon FOWLER 1931 : s./g. de Therapon C. 1817.

Madigania WHITLEY 1945.

Mesopristes BLEEKER 1845: syn. de Datnia C. et V.

Papuservus WhItLey 1943.

Pelates CuVIER 1829.

Pelsartia Whitley 1943.

Plagiogeneion ForBES 1890.

Pseudohelotes GuImarâ̂s 1882.

Pterapon Gray 1833: syn. de Therapon C. 1817.

Rhynchopelates FOWLER 1931: s./g. de Pelates C. 1829.

Scortum WhITLEY 1943.

Terapon CUVIER 1817: vide Therapon C. 1817.

Therapon CUVIER 1817.

FAMILLE DES SCIAENIDAE

(Grondeurs, Tambours, Maigres,

Ombrines ; Corbs ; Croakers ; Roncadores)

Alburnus CATESBY 1771 : non valide. Amblodon RAFINESQUE 1819.

Amblyscion GILL 1863.

Anomiolepis GILL 1861.
Apeches Gistel 1848: syn. de Johnius B. 1793.

Aplodinotus RAFINESQUE 1819.

Argyrosomus DE LA PYLAIE 1835: syn. de Sciaena L. 1758.

Aspericorvina FOWLER 1934: s./g. de Johnius B. 1793.

Asperina OstroumofF 1896: syn. d'Umbrina C. 1817.

Attilus Gistel 1848: syn. d'Um. brina C. 1817.

Bairdiella GILL 1861.

Bola Hamilton-Buchanan 1822.

Callaus Jordan et EIgEnManN 1889.

Cheilotrema TschUDI 1846.

Cheironebris FOWLER 1944.

Chromis (Plumier) DE LACÉPÈdE 1803: non valide.

Cilus Delfin 1900.

Cirrhimens GILL 1862.

Collichthys GüNTHER 1860.

Coracinus Pallas 1811: syn. de Corvina C. 1829.

Corvina CUVIER 1829.

Corvula JoRdAn et EIgENMANN 1889. Cromis BRowne 1789: non valide.

Ctenosciaena FOWLER et BEAN 1923 : s./g. de Sciaena L. 1758.

Diplolepis STEINDACHNER 1863: préemployé, syn. de Plagioscion G. 1861 .

Elattarchus Jop.DAN et EVERMANN 1896.

Eques BLoCH 1793.

Equetus RAFINESQUE 1815: syn. d'Eques B. 1793.

Eriscion JORDAN et EVERMANN 1927. Eutychelithus JORDAN 1876: syn. d'Aplodinotus R. 1819.

Genyonemus GILL 1861.

Haploidonotus GILL 1861: emend. pro Aplodinotus R. 1819.

Hemisciaena BLEEKER 1863: syn. de Collichthys G. 1860.

Homoprion HOLBROOK 1855: syn. de Stellifer (C.) O. 1817.

Johnius BLOCH 1793.

Kroseriphus WHITLEY 1950.

Lamnodon: errat. pro Larimodon (K.) B. 1876 . 
Larimichthys JORDAN et STARKS 1905.

Larimodon (KAUP) BLEEKER 1876: syn. d'Odontoscion G. 1862.

Larimus CUVIER et VAlenCIENNES 1830.

Leiostomus DE LACÉPÈDE 1803.

Lepipterus CUVIER et VALENCIENNES 1830.

Liostomus : errat. pro Leiostomus L. 1803.

Lonchiurus BLOCH 1793.

Lonchurus: vide Lonchiurus B. 1793.

Melantha Gistel 1848: syn. de Corvina C. 1829.

Menticirrhus GILL 1861.

Micropogon CUVIER et VALENCIENNES 1830.

Micropogonias BONAPARTE 1831: syn. de Micropogon C. et V. 1830.

Nector Jordan et Evermann 1898.

Nibea Jordan et Thompson 1911.

Odontoșcion GILL 1862.

Ophioscion GILL 1863.

Othonias JoRdan et THOMPSON 1911.

Pachypops GILl 1861.

Pachyurus Agassiz 1829.

Parabodianus BEAUfort 1945.

Paralarimus Fowler et BEAN 1923.

Paralonchurus BOCOURT 1869.

Pareques GILL 1876.

Pennahia FoWler 1926.

Pinnacorvina FOWLER 1925: s./g. de Cheilotrema T. 1846.

Plagioscion GILL 1861.

Pogonathus (DE LACÉPÈDE) BONAPARTE 1831: syn. de Pogonias L. 1802 .

Pogonias de LACÉPÈde 1802.

Polycirrhus BOCOURT 1896: pré-employé, syn. de Polyclemus B. 1895.

Polyclemus Berg 1895.

Pseudomycterus OGILBY 1908: syn. de Sciaena L. 1758.

Pseudosciaena BLEEKER 1863: syn. de Sciaena L. 1758.
Pseudotolithus BLEEKER 1861.

Pteroscion Fowler 1925: s./g. de Larimus C. et V. 1830.

Rhinoscion Gill 1861: syn. de Cheilotrema T. 1846.

Ronzador Jordan et GILbert 1880.

Scia ena LiNNÉ 1758.

Sciaena Jordan et EVERMANN 1898: syn. de Corvina C. 1829.

Scia snops GILL 1863.

Seriphus AYRes 1857: pré-employé, syn. de Kroseriphus W. 1958.

Sigriurus GILBERT 1898.

Stelicarens JoRDAN et EvERMANN 1898.

Stelififer (CUVIER) OKEN 1817.

Stelliferus StaRK 1828: syn. de Stellifer (C.) O. 1817.

Twi.zonia PASCOE 1908.

Umbrina CUVIER 1817.

Vaczogua Jordan et EvermanN 1927.

Zaclemus GILBeRT 1896.

Zelico WhItLeY 1931.

Zest:dium JORDAN et EVERMANN 1898.

Zest is Jordan et Evermann 1898.

Zonoscion JoRDAN et EVERMANN 1896.

\section{FAMILLE DES GERRIDAE $(=X y$ staemidae $)$ \\ (Mojarras ; Silver-perch)}

Catoshaenum CANTOR 1849: syn. de Gerres (C.) Q. et G. 1824.

Ch:Falmopteryx OGILBY 1887: syn. de Parequula S. 1879.

Clar. GILl 1862: syn. de Pentaprion B. 1850.

Dia $a_{i}$ terus RANZANI 1840.

Eucinostomus BAIRD 1857.

Ger:eomorpha ALLEYNE et MACL.EAY 1877.

Ger:es (Cuvier) Quoy et Gaimard 1824.

Gerres JoRdan et EVERMANN 1898: syn. de Diapterus R. 1840. 
Moharra PoEY 1875.

Mormyrus CATESBY 1771: non valide.

Parequula STEINDACHNer 1879.

Pentaprion BLEEKER 1850.

Pertica Fowler 1904: syn. de Gerres (C.) Q. et G. 1824.

Podager GISTEL 1848: pré-employé, syn. de Gerres (C.) Q. et G. 1824.

Synistius GILL 1862.

Ulaema JoRDAN et EVERMANN 1895. Xystaema Jordan et EVERMANN 1895: syn. de Gerres (C.) Q. et G. 1824.

\section{FAMILLE DES OPLEGNATHIDAE} (incl. Ostorhinchidae)

Hoplognathus GüNTHER : préemployé, syn. d'Oplegnathus R. 1840.

Ichthyorhamphos DE CASTELNAU 1861: syn. d'Oplegnathus R. 1840.

Oplegnathus RicHARDSON 1840.

Ostorhinchus DE LACÉPÈDE 1803.

Scaradon TemmincK et SCHLEgel 1844: vide Scarodon T. et S. 1888.

Scarodon Temminck et Schlegel 1888: syn. d'Oplegnathus R. 1840 .

Scarostoma KNER 1867.

\section{FAMILLE DES MULLIDAE.}

(Rougets, Surmulets ; Surmullets)

Acanthomullus VALENCIENNES 1861.

Atahua PhILlipps 1941.

Barbupeneus WhItLEY 1931.

Brachymullus BLEEKER 1876.

Caprupeneus WHITLEY 1931.

Hogbinia Whitley 1929: s./g. de Pseudupeneus B. 1862.

Hypeneus Agassiz 1846: emend. pro Upeneus C. 1829.

Megalepis BIANCONI 1855: syn. d'Upeneoides B. 1849.
Mullhypeneus POEY 1867.

Mulloides BLEEKER 1849.

Mulloidichthys WHITLEY 1929.

Mullus LINNÉ 1758.

Parupeneus BLEEKER 1863: syn. d'Upeneus C. 1829.

Pennon Whitley 1941.

Pseudomulloides RIBEIRO 1915.

Pseudupeneus BLEEKER 1862.

Upeneichthys BLEEKER 1855.

Upeneoides BLEEKER 1849.

Upeneus CUVIER 1829.

\section{FAMILLE DES BANJOSIDAE}

Anoplus TemmincK et SCHLEgel 1842 : pré-employé, syn. de Banjos B. 1876.

Banjos BLEEKER 1876.

\section{FAMILLE DES LETHRINIDAE}

Heterodon BLEEKER 1845: pré-employé, syn. de Pentapus C. et V. 1830 .

Heterognathodon BLEEKER 1848 : syn. de Pentapus C. et V. 1830. Leiopsis BONAPARTE 1830: syn. de Pentapus C. et V. 1830.

Leiopsis BENNETT 1830: syn. de Pentapus C. et V. 1830.

Lethrinella FOWLER 1904.

Lethrinichthys JORDAN et THOMPSON 1912.

Lethrinus CUVIER 1829.

Maenioides RICHARDSON 1843: syn. de Pentapus C. et V. 1830.

Maina GISTEL 1848 : syn. de Lethrinus C. 1829.

Monotaxis BENNETT 1830.

Pentapodus : emend. pro Pentapus C. et V. 1830.

Pentapus Cuvier et Valenciennes 1830.

Psilopentapodus FOWLER 1933: s./g. de Pentapus C. et V. 1830.

Schour ForSKaL 1775: non valide.

Sphoerodon RüpPELL 1830: syn. de Monotaxis B. 1830. 


\section{FAM. DES NEOLETHRINIDAE}

Neolethrinus DE CASTELNAU 1875.

\section{FAMILLE DES SPARIDAE}

(Dorades, Sargues, Bogues, Pagres,

Pagels, Dentés, Brèmes de mer ;

$$
\text { Pargos) }
$$

Acanthopagrus Peters.

Allotaius WhITLEY 1937.

Archosargus GILL 1865.

Argyrops Swainson 1839: s./g. de Sparus L. 1758.

Argyrozona SмIтн 1938: s./g. de Polysteganus.

Aurata CATESBY 1771: non valide.

Aurata (CUVIER) OKen 1817: syn. de Sparus L. 1758.

Aurata Risso 1826: syn. de Sparus L. 1758.

Austropagrus.

Austrosparus Sмітн 1938.

Axineceps Sмгтн 1938 : s./g. de Polysteganus.

Boridia CUVIER et VALENCIENNES 1830.

Caeso Gistel 1848: syn. de Sparus L. 1758.

Calamus Swainson 1839.

Charax Risso 1826: pré-employé, syn. de Puntazzo B. 1876.

Cheimerius SмIтH 1938.

Chrysoblephus SWAINSON 1839: s./g. de Sparus L. 1758.

Chrysophris Cuvier 1829: syn. de Sparus L. 1758.

Chrysophrys: vide Chrysophris C. 1829.

Cymatoceps Sмгтн 1938.

Cynaedus Gronow 1763: non valide.

Cynaedus (G.) Scopolt 1777.

Datnia Cuvier 1829: syn. de Sparus L. 1758.

Daurada Stark 1828: syn. de Sparus L. 1758.

Denius Gistel 1848 : syn. de Diplodus R. 1810.

Dentex Cuvier 1815.
Diplodus Rafinesque 1810.

Dulosparus Fowler 1933: s./g. de Sparus L. 1758.

Eudynama GisTeL 1848: syn. de Sparus L. 1758.

Evynnis JoRDAN et THOMPSON 1912.

Grammateus POEY 1874.

Labeo BowDICH 1825 : pré-employé, syn. de Labeova W. 1958.

Labeova WHITLEY 1950

Lagodon HolBrooK 1855.

Lithognathus SWAINSON 1839.

Lobodus Costa 1866: syn. de Taenarichthys W. 1953.

Mylio (COMMERSON) DE LACÉPÈDE 1802: non valide.

Nudipagellus FOWLER 1925: s./g. de Sparus L. 1758.

Opsodentex FOWLER 1925: s./g. de Sparus L. 1758.

Otrynter Jordan et Evermann 1896.

Pagellus CuVIER 1828.

Pagrichthys BLEEKER 1860.

Pagrosomus Gill 1893.

Pagrus Cuvier 1817.

Paragyrops : errat. pro Parargyrops T. 1916.

Parargyrops.

Petrus SмITH.

Polysteganus KlunZinger.

Porcostoma SмITH 1938.

Prionosparus Sмттн 1943: s./g. d'Austrosparus S. 1938.

Pterogymnus SмIтн 1938.

Puntazzo BleEker 1876.

Rhabdosargus FOWLER 1933: s./g. de Diplodus R. 1810.

Rhamnubia WHITLEY 1951.

Roughleyia WhItLEY 1931.

Salema Jordan et Evermann 1896. Sargus KLEIN 1775: non valide, syn. de Diplodus R. 1810

Sargus CUVIER 1817: pré-employé, syn. de Diplodus R. 1810.

Semapagrus FoWLeR 1925: s./g. de Pagrus C. 1817.

Sparidentex MUNRo 1948.

Sparodon SмIтH 1938. 
Sparosoma SAUVAGE 1883 : pré-employé, syn. de Rhamnubia W. 1951.

Sparosomus GILL 1893: non valide, syn. de Pagrosomus GILl 1893. Sparus LinNé 1758.

Sphenosargus FOWLER 1940: s./g. de Selema J. et E. 1896.

Stenesthes JoRdAN 1917.

Stenotomus GILL : pré-employé, syn. de Stenesthes J. 1917.

Symphorus GÜNTHER 1872.

Synagris (KLEIN) BLEEKER 1876: syn. de Dentex C. 1815.

Taenarichthys WHITLEY 1953.

Taenarus WhITLEY 1951: syn. de Taenarichthys W. 1953.

Taius JORDAN et THOMPSON 1912.

FAMILLE DES ENOPLOSIDAE

Enoplosus DE LACÉPÈDE 1803.

FAM. DES DIPTERYGONOTIDAE $(=$ Inermiidae $)$

Dipterygonotus BLEEKER 1849.

Inermia POEY 1861.

\section{FAMILLE DES MAENIDAE (Merolepidae)}

Centracantha: vide Centracanthus R. 1810.

Centracanthus RAFINESQUE 1810 .

Centracantus: vide Centracanthus R. 1810.

Coleosmaris NoRMAN 1931.

Maena CUVIER 1829.

Maenas KLEIN 1775: non valide.

Maenas (KLEIN) BLEEKER 1876: syn. de Maena C. 1829.

Marsis Barnard 1927: syn. de Spicara R. 1810.

Merolepis RAFINESQUE 1810: syn. de Maena C. 1829.

Pterosmaris FowLER 1925.

Smaris CuvIER 1815: pré-employé, syn. de Spicara R. 1810.

Smarius Cuvier 1815: syn. de Spicara R. 1810.
Spicata RAFInESQUe 1810.

\section{FAMILLE DES GADOPSIDAE}

Gadopsis RICHARDSON 1844.

\section{FAMILLE DES SCORPIDAE}

(Half-moon fishes)

Agenor de Castelnau 1878: syn. de

Scorpis C. et V. 1831.

Atypichthys GüNTHER 1862.

Atyposoma Boulenger 1899.

Atypus GüNTHER 1860 : pré-employé,

syn. d'Atypichthys G. 1862.

Caesiosoma Kaup 1876: syn. de

Scorpis C. et V. 1831.

Diodyxodon Thомпо 1881.

Helotosoma Kaup 1863: syn. d'Atypichthys G. 1862.

Mecialuna Jordan et FesLer 1893.

Neoscorpis SMITH 1931.

Neatypus WaITE 1905.

Nepotichthys HutToN 1890.

Parepsettus SteIndachner 1875.

Parascorpis BLEEKER 1875.

Scorpis Cuvier et VAlenciennes 1831.

Tilodon THOMINoт 1881.

\section{FAM. DES MONODACTYLIDAE}

Acanthopodus DE LACÉPÈDE 1803 :

syn. de Monodactylus L. 1802.

Centropodus DE LACÉPÈDE 1802:

syn. de Monodactylus L. 1802.

Monodactylus DE LACÉPÈDE 1802.

Psettias JORDAN 1906.

Psetius (COMMERSON) DE LACÉPÈdE 1802: non valide.

Psettus (C.) Cuviter 1829 * syn. de

Monodactylus L. 1802.

Schuettea STEINDACHNER 1866.

FAMILLE DES PLATACIDAE

Platax Cuvier 1817. 


\section{FAMILLE DES EPHIPPIDAE}

Bramichthys WAITE 1905.

Chaetodipterus DE LACÉPÈDE 1803. Ephippus CUVIER 1817.

Ilarches CANTOR 1849: syn. d'Ephippus C. 1817.

Parephippus GILL 1861: syn. de Chaetodipterus L. 1803.

Zabidius WHITLEY 1930.

\section{FAM. DES DREPANICHTHYIDAE} (= Drepanidae)

Cryptosmilia Cope 1867: syn. de Drepanichthys B. 1831.

Drepane Cuvier et VAlENCIENNES 1831: pré-employé, syn. de Drepanichthys B. 1831.

Drepanichthys BONAPARTE 1831.

Enixe Gistel 1848: syn. de Drepanichthys B. 1831 .

Harpochirus CANTOR 1849 : syn. de Drepanichthys B. 1831.

\section{FAMILLE DES TOXOTIDAE}

(Perche-cracheuse)

Amblytoxotes BLEEKER 1876.

Protoxotes WhItLey 1951.

Toxotes CUVIER 1817.

Trompe Gistel 1848 : syn. de Toxotes C. 1817.

\section{FAMILLE DES KYPHOSIDAE} (= Cyphosidae)

(Poissons-pilotes ; Pilot-fishes)

Cridorsa WhITLEY 1938.

Cyphosus: vide Kyphosus L. 1802.

Dorsuarius DE LACÉPÈDE 1803: syn. de Kyphosus L. 1802.

Gregoryina FoWLER et BALL 1924.

Kyphosus de LACÉPÈdE 1802.

Leptokyphosus WHITLEY 1931: s./g. de Segutilum W. 1931.

Opisthistius GILL 1862.

Pimelepterus DE LACÉPÈDE 1803 : syn. de Kyphosus L. 1802.

Sectator JoRdAN et FESLER 1893.

Segutilum WhItLEY 1931.
Seleima Bowdich 1825: syn. de Kyphosus L. 1802.

Tahhmel ForsKaL 1775: non valide. Xyster DE LACÉPÈDE 1803: syn. de Kyphosus L. 1802.

Xysterus RAFINESQUe 1815: syn. de Kyphosus L. 1802.

\section{FAMILLE DES GIRELLIDAE}

(Girelles, Oblades, Grisets, Bogues,

$$
\text { Saupes) }
$$

Aplodon (DumÉrIL) THOMINot 1883 : pré-employé, syn. d'Incisidens G. 1862 .

Boops Cuvier 1815.

Box CuVIER et Valenciennes 1830 : syn. de Boops C. 1815.

Camarina Ayres 1854: syn. de Girella G. 1833 .

Cantharusa STARND 1928: syn. de Spondyliosoma C. 1849.

Cantharus CUvier 1817: pré-employé, syn. de Spondyliosoma C. 1849 .

Caranthus BARNARD 1927: syn. de Spondyliosoma C. 1849.

Coracinus GroNow 1763: non valide.

Crenidens Cuvier et VAlenciennes 1830.

Dichistius GILL 1888.

Dipterodon Cuvier 1829: pré-employé, syn. de Dichistius G. 1888.

Doydixodon VAlencIennes 1855.

Drepanoscorpis FowLER 1934: syn. de Dichistius G. 1888.

Eusalpa FOWLER 1925: remplace Sarpa B. 1831, si non valide.

Exocallus DE LA PYlaIE 1835: syn. de Boops C. 1815.

Girella GraY 1833.

Girellichthys KLUNZINGER 1872: syn. de Melambaphes G. 1863.

Girellipiscis WHITLEY 1931.

Girellops REGAN 1913.

Glyphodes GUICHENOT 1864 : pré-employé, syn. de Guichenotia W. 1950.

Guichenotia WHITLEY 1950. 
Gymnocrotaphus GüNTHER 1859.

Haplodon: errat. pro Aplodon (D.) T. 1883 .

Incisidens GILL 1862.

Iredalella WHITLEY 1931.

Leptometopon SмIтн 1940 : s./g. de Polyamblyodon N. 1935.

Melambaphes GüNTHER 1863.

Melanichthys TeMmincK et SCHLEgeL 1844: syn. de Girella G. 1833.

Neotephraeops DE CASTELNAU 1872: syn. de Melambaphes G. 1863.

Oblada CuVIER 1829.

Pachymetopon GüNTHER 1859.

Polyamblyodon Norman 1935.

Proteracanthus GüNTHER 1859.

Sarpa BONAPARTE 1831: parfois considéré comme non valide.

Scatharus CuVIER et VAlenciennes 1830.

Simocantharus FOWLER 1933 : s./g. de Spondyliosoma C. 1849.

Spondyliosoma CANTOR 1849.

Tephraeops GÜNTHER 1859.

Tripterodon PlayfaIR 1866.

\section{FAMILLE DES SCATOPHAGIDAE}

Cacodoxus Cantor 1849: syn. de Scatophagus C. et V. 1831.

Prenes Gistel 1848: syn. de Scatophagus C. et V. 1831.

Scatophagus CUVIER et VALENCIENNES 1831.

Selenotoca MYERS 1936.

FAM. DES CHAETODONTIDAE

(Papillons-de-mer ; Butterfly-fishes, Coral-fishes)

Acanthochaetodon BLEEKER 1876. Acarauna CATESBY 1771 : non valide. Angelichthys JoRdan et EVERMANN 1896.

Anisochaetodon KLUNZINGER 1884: syn. de Linophora K. 1860.

Apolemichthys BRUNNER 1933: s./g. de Holacanthus L. 1803.
Arusetta BRUNNER 1933.

Centropyge Kaup 1860: syn. de Holacanthus L. 1803.

Chaetodon LINNÉ 1758.

Chaetodontoplus BLEEKER 1876.

Chaetodontops BLEEKER 1876.

Chelmo: vide Chelmon C. 1817.

Chelmon CUVIER 1817.

Chelmon CUVIER 1817.

Chelmonops BLEEKER 1876.

Citharoedus KAUP 1860.

Coradion KauP 1860.

Desmoholacanthus FOWLER 1941.

Desmoprenes FOWLER et BEAN 1929: s./g. de Chaetodon L. 1758.

Diphreutes CANTOR 1849 : syn. d'Heniochus C. 1817.

Eteira Kaup 1860: syn. de Megaprotodon G. 1848.

Euxiphipops FRASER-BRUNNER 1934: s./g. d'Arusetta B. 1933

Forcipiger JoRDAN et MC GREGOR 1898.

Geniacanthus SwaINSON 1839: syn. d'Holacanthus L. 1803.

Genicanthus JoRDAN 1919: syn. d'Holacanthus L. 1803.

Gonochaetodon BLEEKER 1876.

Hemichaetodon BLEEKER 1876.

Hemitaurichthys BLEEKER 1876.

Heniochus CUVIER 1817.

Heteropyge Fraser-BRUNNER 1933 : pré-employé, syn. d'Euxiphipops B. 1934.

Holacantha RAFINESQUE 1815: syn. de Holacanthus L. 1803.

Holacanthus DE LACÉPÈdE 1803.

Lepidochaetodon BLEEKER 1876.

Linophora KAUP 1860.

Loa JoRDAN 1921 : pré-employé, syn. de Roa J. 1923.

Megaprotodon GUICHENOT 1848.

Micracanthus: vide Microcanthus S. 1839.

Microcanthus SWAINSON 1839.

Neochaetodon DE CASTELNAU 1873: syn. de Microcanthus S. 1839. 
Osteochromis FRANZ 1910.

Oxychaetodon BLEEKER 1876.

Paracanthochaetodon SCHMIDT 1930.

Plitops Fraser-BRUnNer 1933 : s./g. de Holacanthus L. 1803.

Pomacanthodes GILL 1862.

Pomacanthops SMITH 1955.

Pomacanthus DE LACÉPÈDE 1803.

Prognathodes GILL 1862.

Pygoplites Fraser-BRUNNer 1933.

Rabdophorus SwaInSon 1839: s./g. de Chaetodon L. 1758.

Rhabdophorus Agassiz 1846 : emend. pro Rabdophorus S. 1839.

Roa JoRDAN 1923.

Sarothrodus GILL 1861: syn. de Chaetodon L. 1758.

Taurichthys CUVIER 1829.

Tetrachaetodon WEBER et BEAUFORT 1936: s./g. de Chaetodon L. 1758 .

Tetragonopterus KLEIN 1775: non valide.

Tetragonopterus (K.) BLEEKER 1868: syn. de Chaetodon L. 1758.

Therapaina KAUP 1860: syn. de Microcanthus S. 1839.

Tholichthys GüNTHER 1868: larve Chaetodon L. 1758.

Tifia JoRDAN et JoRDAN 1923.

Vinculum Mc CULLOCH 1914.

Xiphypops Jordan et Jordan 1923.

FAMILLE DES HISTIOPTERIDAE (Boar-fishes)

Evistias JORDAN 1907.

Evistiopetrus WHITLEY 1932.

Gilchristia JORDAN 1907 : syn. de Pseudopentaceros B. 1876.

Glanertichthys WHITLEY 1945 : s./g. de Paristiopterus B. 1876.

Griffinetta WhItLEY et PHILlipPS 1939.

Histiopterus Temminck et Schlegel 1843.

Maccullochia WaItE 1906: syn. de Paristiopterus B. 1876.
Paristiopterus BleEKER 1876.

Pentaceropsis STEINDACHNER et DöDERLEIN 1883.

Pentaceros Cuvier 1829.

Quadrarius JORDAN 1907.

Quinquarius JORDAN 1907: syn. de Pseudopentaceros B. 1876.

Richardsonia DE CASTELNAU 1872: pré-employé, syn. de Paristiopterus B. 1876.

Undecimus WHITLEY 1934.

Zanclistius JORDAN 1907.

\section{FAMILLE DES NANDIDAE}

Acharnes Müller et Troschel 1848.

Afronandus MeINKen 1955.

Badis BLEEKER 1853.

Bedula Gray 1833: syn de Nandus C. et V. 1831.

Catopra Bleeker 1851 : syn. de Pristolepis J. 1848.

Nandopsis MEIKEN 1954: pré-employé, syn. d'Afronandus M. 1955 .

Nandus CUVIER et VALENCIENNES 1831.

Paranandus Day 1865: syn. de Pristolepis J. 1848.

Pomanotis Guichenot 1847.

Pristolepis JERDON 1848.

FAMILLE DES POLYCENTRIDAE Monocirrhus HECKEL 1840.

Polycentropsis Boulenger 1901.

Polycentrus Müller et Troschel 1848.

FAMILLE DES CICHLIDAE

(Perches des Tropiques)

Acara Heckel 1840: syn. d'Astronotus S. 1839.

Acarichthys EIgENMANN 1912.

Acaronia MYers 1940.

Acaropsis STEINDACHNer 1875: préemployé, syn. d'Acaronia M. 1940.

Aequidens Eigenmann et Bray 1897. 
Amphilophus Agassiz 1858. Apistogramma REgAN 1913. Aristochromis Trewavas 1935. Asprotilapia Boulenger 1901. Astatheros Pellegrin 1904. Astatochromis PeLlegrin 1905. Astatore: errat. pro Astatoreochromis P. 1904.

Astatoreochromis PELLEGRIN 1904. Astatotilapia Pellegrin 1904.

Astronotus SwaINSON 1839.

Aulonocara REgAN 1921.

Aulonocara Trewavas 1935: préemployé.

Aulonocranus Regan 1920.

Baiodon Agassiz 1858: syn. de Theraps G. 1862 .

Bathybates BOULENGER 1898.

Batrachops HeCKEL 1840.

Bayonia BOULENGER 1911.

Biotaecus EIGENMANN et KENNEDY 1903.

Biotodoma EIGENMANN et KENNEDY 1903.

Boggiania Perugia 1897.

Boulengerochromis PELLEgRIN 1904.

Callochromis REGAN 1920.

Callopharynx POLL 1948.

Caquetaia Fowler 1945.

Cardiopharynx POLL 1943.

Chaetobranchopsis EIGENMANN et WARD 1907.

Chaetobranchus HECKEL 1840.

Chaetolabrus Swainson 1839: syn. d'Etroplus C. et V. 1830.

Chaetostoma HeCKEL 1846.

Chaetostomus KNER 1854: emend. pro Chaetostoma H. 1846.

Champsochromis BOULENGER 1916: syn. d'Haplochromis H. 1888.

Chilochromis BOULENGER 1902.

Chilotilapia BOULENGER 1908.

Christiella Trewavas 1935.

Chromichthys DUMÉRIL 1858.

Chromidotilapia BOULENGER 1898.

Chromis CuVIER 1817.
Chromis (C.) GüNTHER 1872: syn. de Tilapia S. 1849.

Cichla Bloch et Schneider 1801.

Cichlasoma SwaInSON 1839.

Cichlaurus SwaInson 1839: syn. de Cichlasoma S. 1839.

Clinodon REgAN 1920.

Cnestrostoma Regan 1920.

Coptodon Gervais 1853.

Corematodus BOULENGER 1896.

Crenicara SteINDACHNER 1875.

Crenicichla Heckel 1840.

Ctenochromis PfeFfer 1893: syn. d'Haplochromis H. 1888.

Cunningtonia BOULENGER 1906.

Cyathochromis Trewavas 1935.

Cyathopharynx REGAN 1920.

Cynotilapia REgAN 1921.

Cyphotilapia REGAN 1920.

Cyrtocara Boulenger 1902.

Dicrossus (Agassiz) SteindachneR 1875.

Diplotaxodon Trewavas 1935.

Docimodus Boulenger 1896.

Ectodus Boulenger 1898.

Enantiopus BOULENGER 1906.

Eretmodus BOULENGER 1898.

Erythrichthys MEEK 1907.

Etroplus Cuvier et VALENCIENNES 1830.

Geophagus HECKEL 1840.

Gephyrochromis BOULENGER 1901.

Gobiochromis POLL 1939.

Gobiocichla KanazaWA 1951.

Grammatotria BOULENGER 1899.

Gymnogeophagus RIBEIRO 1918.

Haligenes GüNTHER 1859: syn. de Tilapia S. 1840.

Haplochromis HILGENDORF 1888.

Haplotaxodon BOULENGER 1906.

Hemibates REgAN 1920.

Hemichromis PeTERS 1857.

Hemitilapia BOULENGER 1902.

Herichthys BAIRD et GIRARD 1854 : syn. d'Astronotus S. 1839.

Heros Heckel 1840: syn. de Cichlasoma S. 1839. 
Heterochromis REGAN 1922.

Heterogramma REGAN 1906 : pré-employé, syn. d'Apistogramma R. 1913.

Heterotilapia Pellegrin 1904.

Heterotilapia REGAN 1920: pré-employé.

Hoplarchus Kaup 1860 : syn. de Cichlasoma S. 1839.

Hoplotilapia STEINDACHNER 1888.

Hygrogonus GüNTHER 186? : syn. d'Astronotus S. 1839.

Hypsophrys Agassiz 1858: syn. d'Astronotus S. 1839.

Julidochromis BOULENGER 1898.

Labeotropheus AHL 1927.

Labidochromis Trewavas 1935.

Labrochromis REGAN 1920.

Labrochromis DAGET 1952: pré-employé.

Lamprologus SCHILTHNIS 1890.

Lepidolamprologus PELLEGRIN 1904.

Leptochromis REGAN 1920.

Leptolamprologus PeLlegrin 1927.

Leptotilapia Pellegrin 1928.

Lestradea POLL 1943.

Lethrinops REGAN 1921.

Lichnochromis TREWAVAS 1935.

Limnochromis REGAN 1920.

Limnotilapia REGAN 1920.

Lipochromis REGAN 1920.

Lobochilotes BOULENGER 1916.

Macropleurodus REGAN 1922.

Melanochromis Trewavas 1935.

Melanogenes BLEEKER 1863.

Mesonauta GÜNTHER 1862.

Mesops GüNTHER 1862: pré-employé, syn. de Biotodoma E. et K. 1903.

Microgaster SwaINSON 1839: syn. d'Etroplus C. et V. 1830.

Mylochromis REgAN 1920.

Nandopsis GILL 1862.

Nannacara REgAN 1905.

Nannacara RibeIRo 1918: pré-employé, syn. de Parvacara W. 1951.
Nannochromis Pellegrin 1904.

Nanochromis JoRDAN 1920: syn. de Nannochromis P. 1904.

Neetroplus GüNTHER 1866.

Neochromis REGAN 1920.

Neopharynx Poll 1948.

Neopharyx: vide Neopharynx P. 1948.

Neotilapia Regan 1920.

Ophthalmotilapia PeLLegRIN 1904.

Oreochromis GüNTHER 1889.

Orthochromis GREENWOOD 1954.

Otopharynx REgAN 1920.

Paracara Bleeker 1878: syn. de Paratilapia B. 1868.

Parachromis Agassiz 1856: syn. de Cichlasoma S. 1839.

Parachromis REgAN 1922.

Paralabidochromis GREENWOOD 1956.

Paraneetroplus REgAN 1905.

Parapetenia REgAN 1905.

Paratilapia BLEEKER 1868.

Parectodus Poll 1943.

Paretroplus B LEEKER 1868: syn. d'Etroplus C. et V. 1830.

Parvacara Whitley 1951.

Pelmatochromis STEINDACHNER 1894.

Perissodus Boulenger 1898.

Petenia GüNTHer 1862.

Petrochromis BoUlenger 1898.

Petrotilapia Trewavas 1935.

Pintoichthys FOWLER 1954.

Plataxoides DE CASTELnAU 1855 : syn. de Pterophyllum H. 1840.

Platytaeniodus BOULENGER 1906.

Plecodus Boulenger 1898.

Pseudetroplus (BLEEKER) GÜNTHER 1862.

Pseudopercis RIBEIRO 1918.

Pseudoplesiops BOULENGER 1899: préemployé, syn. de Nannochromis P. 1904.

Pseudotropheus REGAN 1921.

Pterophyllum HeCKel 1840. 
Ptychochromis STEINDACHNER 1880.

Reganochromis WHITLEY 1929.

Retroculus EIgENMANN et BRAY 1894.

Rhamphochromis REGAN 1921.

Saraca (S.) JORDAN 1920 : vide Saracara S. 1875.

Saracara STEINDACHNER 1875: préemployé, syn. de Biotaecus E. et K. 1903.

Sargochromis REGAN 1920.

Sarotherodon RüPPELL 1852.

Satanoperca GüNTHER 1862.

Schubotzia BoULENGER 1914.

Schwetzochromis POLL 1848.

Serranochromis REGAN 1920.

Simochromis BOULENGER 1898.

Spathodus BoUlenger 1900.

Stappersetta WHITLE 1950.

Stappersia BOULENGER 1914 : pré-employé, syn. de Stappersetta W. 1950.

Steatocranus BoULENGER 1899.

Symphysodon HeCKEL 1840.

Taeniacara MYERS 1935.

Tanganicodus POLL 1950.

Teleogramma BOULENGER 1853.

Telmatochromis BOULENGER 1898.

Theraps GÜNTHER 1862.

Thorichthys MEEK 1904.

Tilapia SмITH 1840.

Tomocichla REGAN 1908.

Trematocara BOULENGER 1899.

Trematocranus TREWAVAS 1935.

Tristramella Trewavas 1942.

Tropheus BOULENGER 1898.

Tylochromis REGAN 1920.

Uaru HeCKel 1840.

Xenochromis BOULENGER 1899.

Xenotilapia BOULENGER 1899.

\section{FAMILLE DES CEPOLIDAE}

Acanthocepola BLEEKER 1874.

Cepola LINNÉ 1764.

Taenia Röse 1793 : pré-employé, syn. de Cepola L. 1764.
FAM. DES HENICICHTHYIDAE Henicichthys TANAKA 1915.

\section{FAMILLE DES CIRRHITIDAE}

Acanthocirrhitus FOWLER 1938.

Amblycirrhitus GILL 1862.

Cirrhites: vide Cirrhitus L. 1803.

Cirrhitichthys BLEEKER 1856.

Cirrhitoidea JENKINS 1903.

Cirrhitops SмIтH 1951.

Cirrhitopsis GILL 1862: syn. de Cirrhitichthys B. 1856.

Cirrhitus DE LACÉPÈde 1803.

Cyprinocirrhites 'TANAKA 1917.

Dactylophora DeVIS 1884.

Gymnocirrhites SмІтн 1951.

Hughichthys SchULtZ 1943.

Isobuna JORDAN 1907.

Lacepedia DE CASTELNAU 1873.

Neocirrhites DE CASTELNAU 1873: syn. de Cirrhitichthys B. 1856.

Oxycirrhites BLEEKER 1857.

Paracirrhites BLEEKER 1875 : syn. de Cirrhitus L. 1803.

Paracirrhites STEINDACHNER et DöDERLEIN 1883 : pré-employé, syn. d'Isobuna J. 1907.

Pseudocirrhites (MOWBRAY) BREDER 1927.

Psilocranium Macleay 1884: syn. de Dactylophora DV. 1884.

FAMILIE DES CHIRONEMIDAE

Chironemus CUVIER 1828.

Sciaenoides (SOLANDER) Richardson 1850: non valide.

Threpterius RICHARDSON 1850.

FAM. DES APLODACTYLIDAE

Aplodactylus Cuvier et VALENCIENNES 1831.

Crinodus GILL 1862.

Dactylosargus GILL 1862.

Goniistius GILL 1862.

Haplodactylus: vide Aplodactylus C. et V. 1831. 
Parhaplodactylus THOMINOT 1883. Zeodrius DE CASTELNAU 1878: syn. de Goniistius G. 1862.

FAM. DES CHEILODACTYLIDAE Acantholatris GILL 1862.

Cheilodactylus DE LACÉPÈDE 1803.

Chirodactylus GILL 1862.

Dactylopagrus GILL 1862: syn. de Sciaenoides B. 1860.

Dactylosparus GILL 1862: non valide, syn. de Dactylopagrus G. 1862.

Morwong WhITLEY 1957.

Nemadactylus RICHARDSON 1839.

Nematodactylus : vide Nemadactylus R. 1839.

Palunolepis BARNARD 1927.

Pteronemus VAN DER HOEVEN 1849: syn. de Cheilodactylus L. 1803.

Sciaenoides BLYTH 1860.

Trichopterus GRONOw 1854 : syn. de Cheilodactylus L. 1803.

\section{Sous-ordre des LABROIDEI}

FAMILLE DES EMBIOTOCIDAE Abeona Girard 1854: syn. de Micrometrus G. 1854.

Amphigonopterus HuBBs 1918.

Amphistichus AgASSIZ 1854.

Brachyistius GILL 1862: s./g. de Micrometrus G. 1854.

Bramopsis (Agassiz) Agassiz 1861 : syn. d'Hyperprosopon G. 1854.

Cymatogaster GiBBons 18 mai 1854. Cymatogaster GiBBons 21 juin 1854 : syn. d'Holconotus A. 1854.

Damalichthys GIRARD 1854.

Ditrema Temminck et Schlegel 1844.

Embiotoca AgASsIZ 1853.

Ennichthys GIRARD 1854 : syn. d'Hyperprosopon G. 1854 .

Holconotus Agassiz 1854.

Hyperprosopon GiBBoNs 1854.

Hypocryptichthys GILL 1862.
Hypsurus Agassiz 1861.

Maenichthys (KAUP) BLEEKER 1876 : non valide, syn. de Ditrema T. et S 1844 .

Metrogaster (AgASSIZ) AGASSIZ 1861 : syn. d'Holconotus A. 1854.

Micrometrus GiBBons 1854.

Microtremus: vide Micrometrus G. 1854.

Mytilophagus GirBons 1854: syn. d'Amphistichus A. 1854.

Neoditrema STEINDACHNER et DöDERLEIN 1883.

Pachylabrus GibBons 1854: syn. de Rhacochilus A. 1854.

Phanerodon GIR ARD 1854.

Rhacochilus AgASsIZ 1854.

Sema JoRdan 1878: syn. de Holconotus A. 1854.

Taeniotoca Agassiz 1861.

Tocichthys HuвBs 1918.

Zalembius JORDAN et EVERMANN 1896.

FAM. DES HYSTEROCARPIDAE

Dacentrus Jordan 1878 : syn. de Hysterocarpus G. 1854.

Hysterocarpus GibBons 1854.

Sargosomus (AgASSIZ) AgASSIZ 1861 : syn. d'Hysterocarpus G. 1854.

\section{FAM. DES POMACENTRIDAE}

Abudefduf ForsKal 1775.

Acanthochromis GILL 1863.

Actinicola Fowler 1904.

Actinochromis.

Agriopopa Whitley 1928.

Amblyglyphidodon BLEEKER 1874. Amblypomacentrus BLEEKER 1877. Ampliprion BLOCH et SCHNEIDER 1801.

Ayresia COOPER 1863.

Azurella JORDAN 1919.

Azurina Jordan et MC Gregor 1898.

Belonochromis FOWLER 1944.

Brachypomacentrus BI EEKER 1877. 
Centrochromis NORMAN 1922.

Cheiloprion WEBER 1913.

Chromis CUVIER 1815.

Chrysiptera SwaINSON 1839: préemployé : syn. d'Iredaleichthys W. 1928.

Ctenoglyphidodon FOWLER 1918.

Dascillus: vide Dascyllus C. 1829.

Dascyllus Cuvier 1829 : pré-employé, syn. de Tetradrachmum C. 1849.

Daya BLEEKER 1877.

Demoisellea Whitley 1928.

Dischistodus GILl 1864: vide Distichodus G. 1863.

Distichodus GILL 1863.

Dorychromis FowLER et BEAN 1928.

Eupomacentrus BLEEKER 1877.

Euschistodus GILL 1862: syn. de Abudefduf F. 1775.

Furcaria PoEY 1860.

Glyphidodon: vide Glyphisodon L. 1803.

Glyphidodontops BLEEKER 1877 : syn. d'Iredaleichthys W. 1928.

Glyphisodon DE LACÉPÈDE 1803: remplace Abudefduf F. 1775 s'il est considéré comme non valide.

Heliases Cuvier 1829: syn. de Chromis C. 1815.

Heliastes: vide Heliases C. 1815.

Hemiglyphidodon BLEEKER 1879.

Heptadecacanthus ALLEYNE et MACLEAY 1877: syn. d'Acanthochromis G. 1863.

Hermosilla JENKINS et EVERMANN 1888.

Homalogrystes All.eYne et Maci.fay 1877: syn. d'Acanthochromis G. 1863 .

Hoplochromis FOWLER 1918.

Hypsipops : vide Hypsypops G. 1861 .

Hypsypops GILL 1861.

Iredaleichthys WHITLEY 1928.

Jerdonia DAY 1870.

Lepicephalochromis FOWLER 1943.
Lepidochromis FOWLER et BEAN 1928.

Lepidozygus GÜNTHER 1862.

Lipophrys GILl 1896 : syn. d'Amphiprion B. et S. 1801 .

Mechaenichthys WHITLEY 1929.

Microspathodon GüNTHER 1862 : syn. de Stegastes J. 1842.

Negostegastes WhITLEY 1929.

Nexilaris: errat. pro Nexilarius G. 1896.

Nexilarius GILBERT 1896.

Nexilosus Heller et SNODgRass 1903.

Oliglyphisodon FOWLER 1941.

Omopomacentrus FOWLER 1944: s./g. de Pomacentrus L. 1803.

Onychognathus Troschel 1866.

Paraglyphidodon BLEEKER 1876.

Paramphiprion WANG 1941.

Parapomacentrus BLEEKER 1877.

Parma GüNTHer 1862

Pellochromis Fowler et BEAN 1928.

Phalerebus WhItLEY 1929.

Pholis Cuvier et VAlenciennes S. 1801 .

Pirene Gistel 1848: syn. de Tetradrachmum C. 1849.

Plectroglyphidodon F OWLER et BALL 1924.

Pomacentrus DE LACÉPÈdF 1803.

Pomataprion GILL 1863: syn. de Stegastes J. 1842.

Premnas CuVIer 1817.

Pristotis RüpPell 1835: syn. de Pomacentrus L. 1803.

Prochilus KLeIN 1775: non valide.

Pseudopomacentrus BLEEKER 1868.

Pterocyclosoma FOWLER 1941.

Pycnochromis FOWLER 1941.

Semadascyllus FOWLER 1941.

Serrichromis FOWLER 1943.

Siphonochromis FOWI ER 1946.

Stegastes JENYNS 1842.

Teixeirichthys SMITH 1953.

Tetradrachmum CANTOR 1849. 
Tetrades BleEker 1865: syn. de Tetradrachmum C. 1849.

Thrissochromis FOWLER 1941.

Wangia FOWI.ER 1954.

Zabuion WhITLEY 1928.

\section{FAMILLE DES LABRIDAE}

(Coquettes, Vieilles, Roucaous, Sublets)

Acantholabrus CUVIER et VALENCIENNES 1839.

Achoerodus GILL 1863.

Anchichoerops BARNARD 1927.

Artisia BEAUFORT 1939

Aspiurochilus FOWLER 1957: s./g. de Lepidaplois G. 1862.

Bodianus BLOCH 1790.

Bodianus CUVIER 1817 : pré-employé, syn. de Harpe L. 1803.

Centrolabrus GüNTHER 1861.

Choerodonoides KAMOHARA 1958.

Choerodon BLEEKER 1847.

Choerops RüPPELL 1852: syn. de Choerodon B. 1847.

Choeropsodes GILCHRIST et THOMPSON 1909.

Choiridon : errat. pro Choerodon B. 1847.

Cichla SCHNEIDER : pré-employé, syn. de Labrus L. 1758.

Cicla KLEIN 1775: non valide.

Cicla Röse 1793 : syn. de Labrus L. 1758.

Clepticus CUVIER 1829.

Coricus Cuvier 1817 : syn. de Crenilabrus C. 1815.

Corycus Cuvier 1815: syn. de Symphodus R. 1810.

Cossyphodes BLEEKER 1860: syn. de Choerodon B. 1847.

Cossyphus Cuvier et VALENCIENnes 1839: pré-employé, syn. de Harpe L. 1803.

Crenilabrus CUVIER 1815.

Ctenocorissa WhItLEY 1931.

Ctenolabrus CUVIER et VALENCIENNES 1839.
Cynaedus Swainson 1839: syn. de Ctenolabrus C. et V. 1839.

Decodon GüNTHER 1862.

Diastodon BowDICH 1825.

Euhypsocara GILL 1863.

Graus PHILIPPI 1887.

Gymnopropoma FowLER et BEAN 1928: s./g. de Lepidaplois G. 1862.

Gymnopropoma GILL 1863: syn. de Lepidaplois G. 1862.

Harpe DE LACÉPÈDE 1803.

Helops BROWNE 1789: non valide, syn. de Bodianus B. 1790.

Heterochoerops STEINDACHNER 1866.

Hiatula DE LACÉPÈDE 1800.

Hospilabrus WHITLE 1931.

Hypsigenys GüNTHER 1861: syn. de Choerodon B. 1847.

Labropsis SснмाDт 1930.

Labrus LINNÉ 1758.

Lachnolaimus CUVIER 1829.

Lachnoloemus: syn. de Lachnolaimus C. 1829.

Lappanella JORDAN 1890.

Lepidaplois GILL 1862.

Lienardella FOWLER et BEAN 1928: s./g. de Lepidaplois G. 1862.

Macrochoerodon FOWLER et BEAN 1928: s./g. de Crenilabrus C. 1815 .

Malacopterus GüNTHER : emend. pro Malapterus C. et V. 1839.

Malapterus CUVIER et VALENCIENNES 1839 : pré-employé, syn. d'Hosvilabrus W. 1931.

Pimelometopon GILL 1864.

Platychoerops KLUNZINGER 1880.

Pseudodax BLEeKer 1861.

Pteragogus Peters 1855.

Rhytejulis FOWLER et BEAN 1928. Ronchifex GISTEL 1848: syn. de Harpe L. 1803.

Scarus Gronow 1763: non valide.

Semicossyphus GÜNTHER 1861.

Suezia SMITH 1957: pré-employé, syn. de Suezichthys S. 1958 
Suezichthys Sмттн 1958.

Suillus CATESBY 1771: non valide, syn. de Lachnolaimus C. 1829.

Symphodus RAFINESQUE 1810.

Tautogolabrus GÜNTHER 1862.

Teleogramma BOULENGER 1899.

Tiricoris WHITLEY 1955.

Torresia dE CASTElnaU 1875: syn. de Choerodon B. 1847.

Trochopus GUiNTHER 1862.

Verreo Jordan et SNYDER 1902.

Verriculus JORDAN et EVERMANN 1903.

Wetmorella FOWI ER et BEAN 1928.

\section{FAMILLE DES CORIDAE}

(Girelles : Rasons)

Cette famille est le plus souvent considérée comme synonyme de la précédente.

Acarauna Sewastianof 1802: syn. de Gomphosus L. 1802.

Amorphocephalus BowDICH 1825: syn. de Xyrichthys C. 1820.

Ampheces JORDAN et SNYDER 1902. Anampses (Cuvier) QuoY et GAIMARD 1824.

Austrolabrus SteIndachner 1884.

Aygula RAFINESQUE 1815: syn. de Coris L. 1802.

Bermudichthys NichOLS 1920.

Cheilinoides BLEEKER 1851.

Cheilinus DE LACÉPÈDE 1802.

Cheilio DE LACÉPÈDE 1803.

Cheiliopsis STEINDACHNER 1863.

Cheilolabrus ALleyne et MACLEAY 1877: syn. de Thalliurus S. 1839.

Chlorichthys Swainson 1839: syn. de Thalassoma S. 1839.

Choerojulis GILL 1862: syn. de Hemiulis S. 1839.

Choeropsodes GILCHRIST et ThомPSON 1909.

Cirrhilabrus Temminck et Schlegel 1845.
Coris de Lacépède 1802.

Crassilabrus SwaINSON 1839: s./g. de Cheilinus L. 1802.

Cymolutes GüNTHER 1862.

Dimalacocentrus GILL 1863 : syn. de Xyrichthys C. 1815.

Diproctacanthus BLEEKER 1862.

Doratonotus GüNTHER 1862.

Dotalabrus WHITLEY 1930.

Duohemipteronotus FOWLER 1957 : s./g. d'Hemipteronotus L. 1802.

Duymaeria BLEEKER 1856.

Elops (COMMERSON) DE LACÉPÈDE 1802: non valide.

Elops (C.) BONAPARTE 1831 : non valide.

Emmeekia JoRDAN et EVERMANN 1896.

Epibulus CUVIER 1815.

Eupemis SwaINSON 1839: syn. de Cheilio L. 1803.

Eupetrichthys RAMSAY et OGILBY 1887.

Fowlerella SмIтH 1957: syn. de Labroides B. 1851.

Gomphosus DE LACÉPÈDE 1802.

Guntheria BLEEKER 1862.

Halichoeres RüPPELL 1835 : pré-employé, syn. de Hemiulis S. 1839.

Halinanodes WHITLEY 1931.

Hemicoris BLEEKER 1862.

Hemigymnus GüNTHER 1861 : syn. de Thalliurus S. 1839.

Hemipteronotus DE LACÉPÈDE 1802. Hemitautoga BLEEKER 1862: syn. de Guntheria B. 1862.

Hemitautoga BLEEKER 1862 : syn. de Coris L. 1802.

Hemijulis: vide Hemiulis S. 1839.

Hemiulis SwaINSON 1839.

Hiatula DE LACÉPÈDE 1800 : pré-employé, syn. de Tautoga M. 1814.

Hinalea JORDAN et JORDAN 1923.

Hologymnosus DE LACÉPÈDE 1802 : syn. de Coris L. 1802.

Ichthycallus SwaINSON 1839: syn. de Julis C. et V. 1815.

Iniistius GILL 1862. 
Iridio JoRDAN et EVERMANN 1896.

Iulis: vide Julis C. 1815.

Julichthys DE VIS 1885.

Julidio JoRDAN et EVERMANN 1896.

Julis CUVIER 1815.

Julis GüNTHER : syn. de Thalassoma S. 1839.

Labrastrum GUICHENOT 1860: syn. de Duymaeria B. 1856.

Labrichthys BLEEKER 1854.

Labroides BLEEKER 1851.

Leptojulis BLEEKER 1862.

Lunolabrus WhITLEY 1933 : s./g. de Pseudolabrus B. 1862.

Macropharyngodon BLEEKER 1862.

Malacocentrus GILI. 1862.

Micropodus RAFINESQUE 1815: syn. de Cheilio L. 1803.

Neanis Gistel 1848: syn. de Julis C. 1815 .

Novacula Cuvier 1817: syn. de Xyrichthys C. 1815.

Novaculichthys BLEEKER 1862: syn. de Xyrichthys C. 1815.

Octocynodon FOWLER 1904.

Ophthalmolepis BLEEKER 1862.

Oxycheilinus GILL 1862.

Oxyjulis GiLL 1863.

Parajulis BLEEKER 1865: syn. d'Hemiulis S. 1839.

Peaolopesia Sмітн 1949.

Pictilabrus GILl 1891.

Platiglossus KLEIN 1775 : non valide.

Platyglossus (K.) BLEEKER 1862: non valide.

Pseudanampses BLEEKER.

Pseudocheilinus BLEEKER 1862.

Pseudocoris BLEEKER 1862.

Pseudojulis BLEEKER 1862.

Pseudojuloides FowLER 1950

Pseudojulops FOWLER 1941.

Pseudolabrus BLEEKER 1862.

Semachlorella FOWLER et BEAN 1928: s./g. de Julis C. 1815.

Stethojulis GüNTHER 1861.

Tautoga MrTCHILL 1814.

Thalassoma SWAINSON 1839.
Thalliurus SwaINSON 1839.

Thysanocheilus KNER 1865.

Urichthys SwaINSON 1839: syn. de Cheilinus L. 1802.

Xenojulis BEAUfORT 1939.

Xiphocheilus BLEEKER 1856.

Xiphochilus

: emend.

pro Xiphocheilus B. 1856.

Xirichthys: vide Xyrichthys C. 1815.

Xyrichthys CUVIER 1815.

Xyrula JORDAN 1890.

\section{FAMILLE DES NEOLABRIDAE}

Neolabrus STEINDACHNER 1875.

\section{FAMILLE DES SPARISOMIDAE}

$$
\text { (= Scarichthyidae) }
$$

Calliodon Cuvier 1829 : pré-employé, syn. de Leptoscarus S. 1839.

Calotomus GILBERT 1890: syn. de Leptoscarus S. 1839.

Cryptotomus CoPE 1871: syn. de

Leptoscarus S. 1839.

Heteroscarus de CASTELNAU 1872.

Leptoscarus SWAINSON 1839.

Vicholsina Fowler 1921: s./g. de Leptoscarus S. 1839.

Scarichthys BLEEKER 1859.

Scaridea JENKINS 1901.

Scarus Cuvier : syn. de Sparisoma S. 1839.

Scarus (FoRSKAL) BI.EEKER 1849: syn. de Sparisoma S. 1839.

Sparisoma SwaINSON 1839.

\section{FAMILLE DES SCARIDAE \\ (= Callyodontidae) \\ (Perroquets-de-mer)}

Amphiscarus SwaInson 1839: syn. de Scarus F. 1775.

Aper (Plumier) de Lać́pède 1803 : non valide, syn. de Scarus F. 1775.

Bolbometopon SMTTH 1956.

Calliodon BLoch et SCHNEIDER 1801 : pré-employé, syn. de Scarus F. 1775. 
Callyodon Gronow 1763: non valide, syn. de Scarus F. 1775.

Callyodon (G.) Scopoli 1777: préemployé, syn. de Scarus F. 1775.

Cetoscarus SмIтH 1956.

Chlorurus SwaInson 1839: syn. de Scarus F. 1775.

Erychthys SwaINSON 1839: syn. de Searus F. 1775.

Euscarus Jordan et EVERMANN 1896. Hemistoma SwaInson 1839: syn. de Scarus F. 1775.

Hipposcarus SMITH 1956.

Loro JoRDAN et EVERMANN 1896

Margaritodon SMITH 1956.

Mormyra BROWNE 1789: non valide.

Novacula CATESBY 1771 : non valide.

Odax (COMMERSON) DE LACÉPÈDE 1802: non valide.

Petronason SwaInSON 1839: svn. de Scarus F. 1775.

Pseudoscarus BLEEKER 1861.

Psittacus CATESBY 1771: non valide.

Scaridea JENKINS 1904.

Scaroides Toula 1909.

Scarops Schultz 1958.

Scarus FoRSKAL 1775.

Xanothon SMITH 1956.

Xenoscarops Schultz 1958: s./g. de Scarops S. 1958.

Xenoscarus Evermann et RadCliffe 1917.

Ypsiscarus Schultz 1958: s./g. de Scarus F. 1775.

\section{FAMILLE DES ODACIDAE}

Coridodax GüNTHER 1862.

Haletta WhITLEY 1947.

Heteroscarus de CASTElnaU 1872.

Neoodax de Castelnau 1875.

Odax CUVIER 1829.

Olistherops GüNTHER : syn. d'Olisthops R. 1850.

Olisthops RICHARDSON 1850.

Sheardichthys WHITLEY 1947 : s./g. de Neoodax C. 1875.
FAM DES SIPHONOGNATHIDAE

Siphonognathus RICHARDSON 1857.

\section{Sous-ordre des TRACHINOIDEI}

\section{FAMILLE DES LATRIDAE}

Latridopsis GILL 1862.

Latris RICHARDSON 1839.

Mendosoma GUICHENOT 1848.

\section{FAM. DES TRICHODONTIDAE}

Arctoscopus JoRDAN et EVERMANN 1896.

Chaetichthys Gistel 1848: syn. de Trichodon (S.) C. 1829.

Trichodon (STELLER) CUVIER 1829.

FAM. DES OPISTHOGNATHIDAE

Gnathypops GILL 1862.

Lonchistium MYERS 1935.

Lonchopisthus GILL 1862.

Merogymnus OGILBY 1908.

Opisthognathus (CUVIER) OK E N 1817.

Stalix JordAN et SNYDER 1902.

Tandya WHITLEY 1930.

Upsilognathus FOWLER 1946.

FAMILLE DES FERCOPHIIDAE

Chriomystax GINSBURG 1955.

Percophis QuoY et GaIMARD 1824.

FAMILLE DES PINGUIPEDIDAE

$(=$ Parapercidae $=$ Mugiloididae $)$

Bathypercis ALCOCK 1893 : pré-employé.

Chilias Ogilby 1910: syn. de Parapercis $\mathrm{B} .1863$.

Macrias GILl et Townsend 1901. Mugiloides DE LACÉPÈDE 1803: syn. de Pinguipes C. 1829.

MyxonUm RAFINESQUE 1815 : syn. de Pinguipes C. 1829. 
Neopercis STEINDACHNER 1885.

Osurus JoRdan et Evermann 1903.

Parapercichthys WhItLEY et PHILLIPS 1939.

Parapercis BLEEKER 1863.

Parapercis STEINDACHNER 1884 : préemployé, syn. de Neopercis S. 1885 .

Percis Bloch et SCHNEIDER 1801: syn. de Pinguipes C. 1829.

Pinguipes CUVIER 1829.

Porteridia FoWLeR 1945.

Pseudopercis RIBEIRO 1903.

Trewara GRIFFIN 1933.

FAMILLE DES PTEROPSARIDAE (= Bembropsidae)

Acanthaphritis GüNTHER 1880.

Bathypercis ALCOCK 1880.

Bembrops STEINDACHNER 1876.

Chrionema Gilbert 1905.

Osopsaron Jordan et STARKS 1904.

Pteropsaron JoRdAN et SNYDER $190 \%$

\section{FAM. DES HEMEROCOETIDAE}

Enigmapercis WHITLEY 1936.

Hemerocoetus CUVIER et VALENCIENNES 1837.

Roxasella FOWLER 1943.

\section{FAMILLE DES \\ CHIMARRICHTHYIDAE}

Chimarrichthys HAAST 1874.

\section{FAMILLE DES CREEDIIDAE}

Apodocreedia BEAUfort 1948.

Creedia OGILby 1898.

Squamicreedia RENDAHL 1921.

FAMILLE DES TRACHINIDAE

(Vives ou Araignées-de-mer)

Corystion RAFINESQue 1810: syn. de Trachinus L. 1758.
Echiichthys BLEEKER 1862.

Hypsicometes GOODE 1881.

Pseudotrachinus BLEEKER 1862.

Trachinus LINNÉ 1758.

\section{FAM. DES LIMNICHTHYIDAE}

Chalixodes: errat. pro Chalixodytes S. 1943.

Chalixodytes Schultz 1943.

Limnichthys WAITE 1904.

Schizochirus WaIte 1904.

FAMILLE DES LEPTOSCOPIDAE Leptoscopus GILL 1859.

Matsubaraea TAKI 1953.

FAMILLE DES TRICHONOTIDAE

Crystallodytes FOWLER 1923.

Gobiotrichonotus FOWLER 1943.

Kraemeria STEINDACHNER 1906.

Lesueuriella FOWLER 1907: syn. de Lesueurina F. 1907.

I.esueurina FOWLER 1907.

Parkraemeria WhItLeY 1952.

Trichonotus BLOCH et SCHNEIDER 1801.

Taeniolabrus STEINDACHNER 1867: syn. de Trichonotus B. et S. 1801 .

Tewara GRIfFin 1933.

FAMILLE DES OXUDERCIDAE

Oxuderces VALENCIENNES 1842.

FAM. DES BATHYMASTERIDAE

Bathymaster COPE 1873.

Rathbunella JoRDAN et EvermanN 1896.

Ronquilus Jordan et SNYDER 1895.

FAMILLE DES ZAPRORIDAF:

Zaprora JORDAN 1896. 
FAMILLE DES URANOSCOPIDAE (Rat, Tapecon, Rascasses blanches)

Agnus GüNther 1860: syn. d'Astroscopus B. 1860

Anema GüNTHER 1860: syn. d'Ichthyoscopus S. 1839.

Ariscopus Jordan et SNYDER 1902. Astroscopus Brevoort 1860.

Benthoscopus LONGLEY et HILDEBRAND 1941.

Cathetostoma GiLl 1861 : vide Kathetostoma G. 1860.

Execistides JORDAN et THOMPSON 1905.

Genyagnus GILL 1861.

Gnathagnoides WHITLEY et PHILLIPPS 1939.

Gnathagnus GILI. 1861.

Ichthyoscopus SwaINSON 1839.

Kathetostomus GüNTHER 1860

Nematagnus GILL 1861.

Pleuroscopus BARNARD 1927.

Synnema HAAST 1873.

Upsilonphorus GILL 1861 : syn. d'Astroscopus B. 1860.

Uranoscopus LINNÉ 1758.

Zalescopus Jordan et HubBs 1925.

\section{FAMILLE DES OWSTONIIDAE} (parfois réunie aụx Cepolidae)

Oustonia TANAKA 1908.

Pseudocepola Kamohara 1935.

FAM. DES CHAMPSODONTIDAE

Centropercis OgILby 1895.

Champsodon GÜNTHER 1867.

FAM. DES HARPAGIFERIDAE

Harpagifer RICHARDSON 1844.

Sclerocottus FISCHER 1885.

\section{FAMILLE DES BOVICHTHIDAE}

Bovichthus: vide Bovictus C. et V. 1831.
Bovichthys: vide Bovictus C. et V. 1831.

Bovictus Cuvier et VALENCIENNES 1831.

FAM. DES CHAENICHTHYIDAE $(=$ Channichthyidae $)$

Chaenichthys RICHARDSON 1844.

Chaenocephalus REGAN 1913.

Chaenodraco REGAN 1914.

Champsocephalus GILL 1861.

Channichthys RICHARDSON 1844 : syn. de Chaenichthys R. 1844.

Chionodraco.

Cryodraco DoLlo 1900.

Dacodraco WAITE 1916.

Neopagetopsis NYBELIN 1947.

Pagetodes RICHARDSON 1844: * non valide, pas de nom d'espèce, et le type a été emporté par un chat (sic), JORDAN 1919 ».

Pagetopsis Regan 1913.

Parachaenichthys BOUI ENGER 1902.

Ponerodon ALCOCK 1390.

Pseudochaenichthys NORMAN 1937.

FAM. DES BATHYDRACONIDAE Aconichthys WAITE 1916.

Bathydraco GüNTHER 1878.

Cygnodraco WaITe 1916.

Gerlachea Dollo 1900

Prinodraco REgan 1914.

Psilodraco NORMAN 1937.

Racovitzia Dollo 1900: syn. d'Aconichthys W. 1916.

FAMILLE DES NOTOTHENIIDAE

Aphritis Cuvier et Valenciennes 1831: pré-employé, syn. de Phricus B. 1895.

Artedidraco LÖNINBERG 1905.

Aurion WaITE 1916.

Cottoperca SteIndachner 1876.

Dissostichus Sмттт 1898.

Dolloidraco RoUI.E 1913. 
Eleginops GILL 1861.

Eleginus CuVIER et VALENCIENNES 1830: pré-employé, syn. d'Eleginops G. 1861.

Gelidus WHITLEY 1937.

Histriodraco REGAN 1914.

Macronotothen GILL 1861.

Notothenia RICHARDSON 1844.

Pagothenia Nıchols et LA MONTE 1936.

Phricus Berg 1895.

Pleuragramma Boulenger 1902 : syn. de Gelidus W. 1937.

Pogonophryne REgAN 1914.

Pseudaphritis DE CASTELnAu 1872.

\section{Sous-ordre des CHIASMODONTOIDEI}

FAM. DES CHIASMODONTIDAE

Chiasmodon JoHNSON 1863.

Chiasmodus GüNTHER : emend. pro Chiasmodon J. 1863.

Dolichodon PARR 1931.

Dysalotus Mc GILCHRIST 1905.

Hemicyclodon PARR 1931.

Kali Lloyd 1909.

Myersiscus FOWLER 1934.

Odontonema WEBER 1913.

Pseudoscopelus LÜTKeN 1892.

\section{Sous-ordre des CALLIONYMOIDEI}

FAMILLE DES CALLIONYMIDAE

(Dragonnets, Doucets. Chiqueurs)

Brachycallionymus HERRE et MYERS 1936.

Callimucenus WhItLEY 1934.

Callionymus LINNÉ 1758.

Calliurichthys JORDAN et FOWLER 1903: s./g. de Callionymus L. 1758.
Calymmichthys JORDAN et THOMPSON 1914.

Dactylopus GLL 1859.

Dermosteira Schultz 1943.

Dicallionymus Fowler 1941.

Draculo SNYDER 1911.

Foetorepus WHITLEY 1931.

Orbonymus WhitLey 1947.

Paracallionymus BARNARD 1927.

Pogonymus Gosi.ine 1959.

Repomucenus WHUTI.EY 1931.

Synchiropus GILL 1859.

Velesionymus WHITLEY 1934.

Vulsus Güinther 1861: syn. de Dactylopus G. 1859.

Yerutius WhITLEY 1931.

FAM. DES DRACONETTIDAE

Centrodraco REgAN 1913.

Draconetta JORDAN et FOWLER 1.903.

\section{Sous-ordre des BLENNIOIDEI}

\section{FAMILLE DES BIENNIIDAE}

(Cagnotte, Mordocet ou Morde-au-sec Baveuse)

Adonis Gronow 1854: syn. de Blennius L. 1758.

didablennius WHITLEY 1947.

Alticops SмIтн 1948.

Alticus (COMMERSON) BLEEKER 1869: syn. de Rupiscartes S. 1839.

Alticus (C.) DE LACÉPÈDE 1800 : non valide.

Antennablennius FOWLER 1931: s./g. de Blennius L. 1758.

Aspidontus QuOY et GaImard 1834. Atrosalarias WhitLey 1933.

Basilisciscartes FOWLER 1939: s./g. de Salarias C. 1817.

Blennechis CUVIER et VALENCIENNES 1836: syn. de Petroscirtes R. 1828.

Blenniella REID 1943. 
Blenniolus JORDAN et EVERMANN 1898.

Blennitrachus SwaInson 1839: syn. de Chasmodes C. et V. 1836.

Blennius LINNÉ 1758.

Blennophis VALENCIENNES 1844 : préempioyé, syn. d'Ophioblennius G. 1860 .

Blennus KLEIN 1779: non valide.

Brachynectes ScotT 1957.

Brannerella GILBERT 1900.

Calliblennius Aoy.ıgI 1954: pré-employé,

Chalaroderma NORMAN 1943.

Chasmodes CUVIER et VALENCIENNES 1836.

Cirripectes SwaInson 1839: syn. de Rupiscartes S. 1839.

Coryphoblennius NoRMANN 1943.

Crenalticus WhtrLeY 1930.

Croaltus SмITH 1959.

Cruantus Sмrтн 1959.

Cyneichthys OGILBY 1910: s./g. de Petroscirtes R. 1828.

Cynoscartes Norman 1943 : s./g. de Scartichthys J. et E. 1898.

Dasson Jordan et HubBs 1925.

Dubiblennius WhItLEY 1930.

Ecsenius Mc Culloch 1923.

Enchelyurus PETERS 1868.

Entomacrodops FOWLER 1944.

Entomacrodus GILL 1859.

Erpichthys SwaINSON 1839: syn. de Salarias C. 1817.

Escadodus Sмттн 1959: s./g. d'Aspidontus Q. et G. 1834 .

Exallias JoRdAN et EVERMANN 1905.

Forsterygion WhITLEY et PhILLIPPS 1939.

Giffordella FOWLER 1932

Gilloblennius WhITLEY et PHILLIPPS 1939.

Gloriella Schultz 1941.

Glyptoparus SмIтH 1959.

Graviceps FOWLER 1903.

Halmablennius SMITH 1948.

Hepatoscartes FOWLER 1944.
Hirculops SмIтH 1959.

Homesthes GILBERT 1898.

Hypleurochilus GILL 1861.

Hypopleurochilus: vide Hypleurochilus G. 1861.

Hypsoblenniops SchULTZ 1943.

Hypsoblennius GILL 1861.

Ichthyocoris BONAPARTE 1836: syn. de Blennius L. 1758.

Isesthes JORDAN et GILBERT 1882: syn. d'Hypsoblennius G. 1861.

Istiblennius WHITLEY 1943.

Laiphognathus SмIтH 1956.

Lambeichthys HERRE 1936.

Latroblennius BORODIN 1928.

Leoblennius REID 1943.

Lipophrys GILL 1896.

Litanchus SMITH 1959: s./g. de Blennius L. 1758 ou d'Antennablennius F. 1931.

Lophalticus SмIтн 1957.

Lucaya BöHLKE 1957: pré-employé, syn. de Lucayablennius B. 1958.

Lucayablennius BöHLKE 1958.

Lupinoblennius HERRE 1943.

Macrurrhynchus OGILBY 1896: syn. d'Aspidontus Q. et G. 1834.

Meiacanthus NORMAN 1943.

Meiacanthus Томтуама 1956: préemployé,

Negoscartes WHITLE 1930.

Nixiblennius WHITLEY 1930.

Omobranchus (EHRENBERG) CUVIER et VAlenciennes 1836: syn. d'Aspidontus Q. et G. 1834.

Oncesthes JORDAN et HUBBS 1925.

Ophioblennius GILL 1860.

Ostreoblennius WHITLEY 1930 : s./g. de Petroscirtes R. 1828.

Parablennius RIBEIRO 1915.

Pauloscirtes WhItLEY 1935.

Pereulixia SMITH 1959.

Pescadorichthys Tомтуама 1955.

Petroscirtes RüPPEll 1828.

Pholis Cuvier 1817: pré-employé, syn. de Lipophrys G. 1896. 
Pictiblennius Whitley 1930.

Poroalticus FOWLER 1931.

Queriblennius WhITLEY 1933 : s./g. de Blennius L. 1758.

Rhabdoblennius WHITLEY 1930.

Rupiscartes SwaInson 1839.

Salaria FoRSKAL 1775: syn. d'Aidablennius W. 1947.

Salarias CUVIER 1817.

Salarichthys GUICHENOT 1867.

Salariichthys: vide Salarichthys G. 1867.

Scartella JORDAN 1886.

Scartes JoRdAN et EvermanN 1896: pré-employé, syn. de Scartichthys J. et E. 1898.

Scartichthys JORDAN et EVERMANN 1898.

Scartoblennius FOWLER 1946.

Semablennius FOWLER 1954.

Soldatovia TARANETZ 1958.

Somersia BeEbe et Tee-Van 1934.

Spinoblennius HERRE 1935.

Stanulus Sмгтн 1959.

Tagusa Herre 1935.

Tekla Nichols 1922.

Xenopholis Davis 1887 : pré-employé, syn. de Xenopholoides F. 1958 .

Xenopholoides FoWLER 1958.

Zeablennius WHITLEY 1930.

FAMILLE DES PARACLINIDAE nom. nov. (= Acanthoclinidae)

Acanthoclinus MocQuard 1885 : préemployé, svn. de Paraclinus M. 1888.

Paraclinus MocQUARD 1888.

\section{FAM. DES ANARHICHADIDAE}

(Chat marin ou Loup marin)

Anarchichas JORDAN 1923 : errat. pro Anarhichas L. 1758.
Anarhichas LINNÉ 1758.

Anarrhichas ARTEDI 1793 : errat. pro Anarhichas L. 1758.

Latargus : errat. pro Lathargus $\mathrm{K}$. 1775.

Lathargus KLEIN 1775: non valide. Lycichthys GILL 1877.

\section{FAMILLE DES CLINIDAE}

(Gonnelles ou Papillons-de-mer)

Acteis Jordan 1904.

Alloclinus HuBBs 1927.

Andracanthus LONGLEY 1928.

Auchenionchus GILL 1860.

Auchenipterus : errat. pro Auchenopterus G. 1861.

Auchenopterus GüNTHER 1861: préemployé, syn. de Cremnotekla W. 1940 .

Axoclinus FOWLER 1944.

Blakea STEINDACHNER 1876: syn. de Gibbonsia C. 1863.

Blennioclinus GILL 1860.

Blenniomimus SмIтн 1946.

Blennophis SwaInson 1839.

Brockius Hubss 1953 : s./g. de Labrisomus S. 1839.

Calliblennius BARBOUR 1912.

Calliclinus GILL 1860.

Cirrhibarbus CUVIER 1829.

Climacoporus BARNARD 1935

Clinitrachus SwaInson 1839: syn. de Cristiceps C. et V. 1836

Clinoides GILCHRIST et THOMPSON 1908.

Clinoporus BARnARD 1927.

Clinus CUVIER 1817.

Cologrammus GILL 1893.

Congrammus FoWler 1906.

Corallicola JORDAN et EverMANN 1898.

Coralliozetus Evermann et MARSH 1899.

Cremnobates GüNTHER 1861: préemployé, syn. de Cremnotekla W. 1940.

Cremnotekla WhitLey 1940.

ANN. PARASITO. HUM. ET COMP., 1962, 37, $\mathrm{n}^{\circ} 6^{\text {bis }}$ (fasc. suppl.) 
Cristiceps Cuvier et Valenciennes 1836.

Crockeridius CLARK 1936.

Cryptotrema GILBeRT 1890.

Ctenichthys RIVERO 1936.

Dialommus GILBERT 1890.

Emmnion JORDAN 1897.

Enneanectes JorDAN et GILBERT 1895.

Enneapterygius RüPPELL 1835.

Entomacrodus GILL 1859.

Ericentrus GILL 1893.

Ericteis JORDAN 1904.

Exerpes Jordan et EVERMANN 1896.

Fucomimus Sмітн 1946.

Gibbonsia COOPER 1863.

Gillias EvermanN et Marsh 1899.

Gobioclinus GILL 1860.

Gynutoclinus SмIтH 1946.

Helcogramma Mc CuLloch et WAITE 1918.

Heterostichus GIRARD 1854.

Histioclinus MetzelaAR 1921.

Labrisoma: vide Labrisomus S. 1839.

Labrisomus SwaINSON 1839.

Labroclinus Sмттн 1946.

Labrosomus: vide Labrisomus S. 1839.

Lepidoblennius STEINDACHNER 1867.

Lepidoblennius SAUVAGE 1874: préemployé, syn. de Sauvagea J. et S. 1906.

Lepisoma Dekay 1842: pré-employé, syn. de Labrisomus S. 1839.

Malacoctenus GILL 1860.

Mnierpes JoRdAN et EVERMANN 1896.

Muraenoclinus SMITH 1946.

Myersichthys HuBss 1952.

Myxodes CUVIER 1829.

Neoclinus GIRARD 1858.

Norfolkia FOWLER 1953.

Notoclinops WhItLey 1930.

Notoclinus GILL 1893.
Odontoclinus REID 1935: s./g. de Labrisomus S. 1839.

Ophthalmolophus GILL 1860.

Paracristiceps HeRre 1939.

Parviclinus FRASER-BRUNNER 1932.

Pavoclinus SMITH 1946.

Petraites OgILby 1885.

Petrognathus (GIRARD) JoRdan et EVERMANN 1898.

Petrognathus GIRARD 1859: non valide.

Phillippsichthys HuBBs 1952.

Sauvagea JoRdAN et SEALE 1906.

Smithichthys HuвBs 1952.

Starksia JoRdan et EVERMANN 1896.

Tekla Nichols 1922.

Trianectes Mc Culloch et WatTE 1918.

Tripterygion Risso 1826.

Tripterygium: vide Tripterygion $\mathbf{R}$. 1826.

Vauclusella WhITLEY 1931.

Verconectes WHITLEY 1931.

Xenopoclinus SмIтH 1948

Zacalles JORDAN et SNYDER 1902: pré-employé, syn. de Calliblen. nius B. 1912.

\section{FAM. DES EMBLEMARIIDAE}

Acanthemblemaria MetzelaAR 1920.

Coralliozetus EvermanN et MARSH 1899: syn. d'Emblemaria J. et G. 1882 .

Emblemaria JORDAN et GILBERT 1882.

Emblemariopsis LONGLEY 1928.

Hemiemblemaria LONGLEY et HILDEBRAND 1941.

Paremblemaria LONGLEY 1928.

Psednoblennius Jenkins et EvermanN 1888: syn. d'Emblemaria J. et G. 1882 .

FAMILLE DES RUNULIDAE

Andamia BLyth 1859. 
Damania SмrTH 1959.

Heteroclinus de Castelnau 1872.

Runula JoRdAN et Bollman 1889.

Runulops Ogilby 1910: s./g. de Runula J. et B. 1889.

FAMILLE DES ATOPOCLINIDAE Atopoclinus Vaillant 1894.

FAMILLE DES CHAENOPSIDAE Auchenistius Evermann et Marsh 1899: s./g. de Stathmonotus B. 1885 .

Chuenopsis GILL 1863.

Lucioblennius GILBERT 1890.

Parastathmonotus CHABANA UD 1942.

Pholidichthys BLEEKER 1856.

Stathmonotus BEAN 1885.

FAM. DES CEBIDICHTHYIDAE

Cebedichthys: vide Cebidichthys A. 1855.

Cebidichthys AYres 1855.

Neozoarces STEINDACHNER 1880.

Zoarchias JORDAN et SNYDER 1902.

\section{FAMILLE DES STICHAEIDAE}

Cryptacanthoides SOLDATOV et LINDBERG 1930.

Dinogunnellus HERZENSTEIN 1890.

Ernogrammus JORDAN et EVERMANN 1898.

Eumesogrammus GILL 1864.

Kasatkia Soldatov et PavlenKo 1923.

Notogrammus Bean 1881: syn. de Stichaeus R. 1837.

Ozorthe JORDAN et EVERMANN 1898. Stichaeopsis STEINDACHNER et KNER 1870.

Stichaeus REINHARDT 1837.

Ulvarius JORDAN et EVERMANN 1896.
FAMILLE DES LUMPENIDAE Acantholumpenus MAKUSHOK 1958. Allolumpenus HUBBS et SCHULtZ 1932.

Anisarchus GILL 1864.

Centroblennius GILL 1861.

Ctenodon NILsson 1855: pré-employé, syn. de Leptoclinus G. 1861.

Leptoblennius GILL 1860.

Leptoclinus GILL 1861.

Leptogunnellus AYRES 1855 : syn. de Lumpenus R. 1837.

Lumpenella HUBBS 1927.

Lumpeneopsis Soldatov 1923.

Lumpenus REINHARDT 1837.

Poroclinus BeAN 1890.

FAMILLE DES XIPHISTERIDAE (= Xiphidiontidae)

Epigeichthys HUBBS 1927.

Phytichthys HUBвs 1923.

Xhiphidion GIRARD 1857: pré-employé, syn. de Xiphister J. 1879.

Xiphister JORDAN 1879.

Xiphistes Jordan et Starks 1895.

FAMILLE DES PTILICHTHYIDAE

Ptilichthys BEAN 1881.

FAMILLE DES

CRYPTACANTHODIDAE

Cryptacanthodes STORER 1839.

Delolepis BEAN 1881.

Lyconectes GILBERT 1895.

FAMILLE DES PHOLIDAE

(Papillons-de-mer)

Abryois JORDAN et SNYDER 1902.

Alectrias Jordan et EvermanN 1898.

Alectridium GILBERT et BURKB 1912.

Anoplarchus GILl 1861.

Apodichthys GIRARD 1854. 
Askoldia PavlenKo 1910.

Asternopteryx (RüPPELL) GüNTHER 1861.

Azuma Jordan et SNYDER 1902.

Blenniops NiLsson 1855: syn. de Chirolophis S. 1839.

Blennophidium BOULENGER 1892 : syn. d'Opisthocentrus K. 1868.

Bryolophus JORDAN et SNYDER 1902: pré-employé, syn. de Bryozoichthys W. 1931.

Bryostemma JoRdAN et STARKS 1895 : syn. de Chirolophis S. 1839.

Bryosoichthys WHITLEY 1931.

Carelophus KRÖYER 1845: syn. de Chirolophis S. 1839.

Centronotus BLOCH et SCHNEIDER 1801: syn. de Pholis S. 1777.

Chirolophis SwaINSON 1839.

Dactyleptus RAFINESQUE 1815.

Dictyosoma Temminck et Schlegel 1845.

Enedrias JORDAN et EVERMANN 1898.

Eulophias SмIтH 1902.

Gunnellops BLEEKER 1874

Gunnellus Cuvier et VALENCIENNES 1836: syn. de Pholis S. 1777.

Gymnoclinus GILBERT et BURKE 1912.

Muraenoides DE LACÉPÈDE 1800: syn. de Pholis S. 1777.

Ophisomus SwaInson 1839: syn. de Pholis S. 1777.

Opisthocentrus KNER 1868.

Pholidapus BEAN et BEAN 1896. Pholis GRONOw 1763: non valide

Pholis SCOPOLI 1777.

Pholis RösE 1763.

Plagiogrammus BEAN 1893.

Plectobranchus GILBERT 1890.

Rhodymenichthys JORDAN et EVERMANN 1896.

Trigrammus Gracianov 1907.

Ulvicola GILBERT et STARKS 1897.

Urocentrus KNER 1868: syn. de Pholis S. 1777.

Xererpes Jordan et GILBERT 1895.
FAMILLE DES ANARRHICHTHYIDAE

Anarrhichthys AYRES 1855.

FAMILLE DES XIPHASIIDAE

Nemophis Kaup 1858: syn. de Xiphasia S. 1839.

Plagiotremus GILL 1863.

Xiphasia SwaInson 1839.

Xiphogadus GüNTHer 1862: syn. de Xiphasia S. 1839.

Ziphasia SwaInSON 1839 : errat. pro Xiphasia S. 1839.

FAM. DES XENOCEPHALIDAE

Xenocephalus KAUP 1858.

FAMILLE DES HALIOPHIIDAE

Halimuraena SMITH 1952.

Haliophis RüPPELL 1828.

FAM. DES CONGROGADIDAE

Blennodesmus GüNTHER 1871.

Congrogadoides BORODIN 1933.

Congrogadus GüNTHER 1862.

Halidesmus GÜNTHER 1871.

Hierichthys JORDAN et FOWI ER 1902:

syn. de Congrogadus G. 1862.

Machaerium RICHARDSON 1843 : préemployé, syn. de Congrogadus G. 1862 .

Programmus Gilchrist et Thompson 1916: syn. de Halidesmus G. 1871

FAMILLE DES GUNNELLICHTHYIDAE

Clarkichthys Sмітн 1958: s./g. de Gunnellichthys B. 1858.

Gunnellichthys BLEEKER 1858.

FAM. DES MICRODESMIDAE $(=$ Cerdalidae $)$

Cerdale Jordan et Gilbert 1881. 
Leptocerdale WeYMOUTH 1910. Microdesmus GÜNTHER 1864.

FAM. DES SCYTALISCIDAE nom. nov. (= Scytalinidae)

Scytalina JORDAN et GILBERT 1880 : pré-employé, syn. de Scytaliscus J. et G. 1883 .

Scytaliscus Jordan et GILBERT 1883.

\section{FAMILLE DES ZOARCIDAE (Loquette)}

Allolepis Jordan et HubBs 1925.

Aprodon Gilbert 1890.

Austrolichthys REGAN 1913.

Austrolycus Regan 1913.

Bassozetus GILL 1883.

Bilabria ScHMIDT 1936.

Bothrocarichthys ScHMIDT 1938.

Bothrocarina Suvorov 1935.

Commandorella TARANETZ et ANDRIASHEV 1935.

Crossolycus Regan 1913.

Dadynos WhITLEY 1951.

Davidijordania Popov 1931.

Derjugina Popov 1931.

Embry $\boldsymbol{x}$ JoRdan et EVERMANN 1898.

Enchelyopus Gronow 1763 : non valide, syn. de Zoarces C. 1829.

Eutyx Heller et SNodgrass 1903. Furcella Jordan et EvermanN 1896: pré-employé, syn. de Furcimanus J. et E. 1898.

Furcimanus JoRDAN et EVERMANN 1898.

Gymnelichthys FISCHER 1885.

Gymnelis REINHARDT 1838.

Gymnelopsis Soldatov 1923.

Hadropareia ScHмIDT 1904.

Hypolycodes Hector 1880.

Iluocoetes JENYNS 1842

Krusensterniella ScHMIDT 1904.

Leurynnis LOCKINGTON 1880 : syn. de Lycodopsis C. 1879.

Lycenchelys GILL 1884.
Lycias Jordan et EVERMANN 1898.

Lycocara GILL 1884.

Lycodalepis BLEEKER 1874.

Lycodes REINHARDT 1838.

Lycodophis VaILlant 1888: syn. de Lycenchelys G. 1884.

Lycodopsis COLLETT 1879.

Lycogramma GILBERT 1915.

Lycrogrammoides S O D A T O V et LINDBERG 1929.

Lyconema GILBERT 1895.

Lycozoarces PoPOV 1935.

Macrozoarces GILL 1863.

Mastigopterus RADCLIFFE 1913.

Maynea Cunningham 1871.

Melanostigma GÜNTHER 1881.

Nemalycodes HeRTZENSTEIN 1896.

Oculospinus KOEFOED 1928.

Ophthalmolycus REGAN 1913.

Pachycara ZUGMAYER 1911: pré-employé, syn. de Pachycarichthys W. 1931.

Pachycarichthys WHITLEY 1931.

Paralycodes BleEKER 1874.

Petroschmidtia TARANetz 1934.

Petrotyx Heller et Snodgrass 1903.

Pholidus RAfINESQUe 1815: syn. de Zoarces C. 1829.

Phycocoetes JENYNS 1842.

Platea STEINDACHNER 1898: pré-employé, syn. de Dadynos W. 1951.

Pogonolycus Norman 1937.

Pseudobythites MEEK et HILDEBRAND 1928 : syn. de Petrotyx H. et S. 1903.

Pterotyx : errat. pro Petrotyx H. et S. 1903.

Selachophidium GILCHRIST 1903.

Uronectes GüNTHER 1862: pré-employé, syn. de Lycocara G. 1884.

Zestichthys JoRDAN et HuBBS 1925.

Zoarces CUVIER 1829.

Zoarchus: syn. de Zoarces C. 1829.

Zoarcites ZUGMAYER 1914. 


\section{FAMILLE DES DEREPODICHTHYIDAE}

Derepodichthys GILBERT 1895.

\section{FAM. DES LYCODAPODIDAE}

Lycodapus GILBERT 1890.

Snyderidia GILBERT 1905.

\section{FAM .DES NOTOGRAPTIDAE}

Blanchardia DE CASTELNAU 1875: syn. de Notograptus G. 1867.

Notograptus GüNTHER 1867.

Sticharium GüNTHER 1867.

FAMILLE DES PERONEDYIDAE

Eucentronotus OGILBY 1898: syn. de Peronedys S. 1884.

Peronedys Steindachner 1884.

\section{FAMILLE DES OPHIOCLINIDAE}

Neoblennius de CASTELnaU 1875.

Neogunellus DE CASTELAN 1875: syn. d'Ophioclinus C. 1872.

Ophioclinops WhITLEY 1932.

Ophioclinus de CASTElnau 1872.

Scleropteryx Devis 1894 : non valide, syn. d'Ophioclinus C. 1872.

Stenophus DE CASTELNAU 1875.

FAM. DES DACTYLOSCOPIDAE

Cokeridia MeEk et Hildebrand 1928.

Crapatalus GüNTHER 1861.

Dactylagnus GILL 1862.

Dactyloscopus GILL 1859.

Esloscopus JoRDAN et EVERMANN 1896.

Gillellus GILBERT 1890.

Heteristius MYERS et WADE 1946.

Myxodagnus GILL 1861.
FAMILLE DES LEPTOSCOPIDAE Crapatulus GII L 1861 : pré-employé, Leptoscopus GILL 1859.

\section{Sous-ordre des OPHIDIOIDEI}

FAMILLE DES BROTULIDAE

Acanthonus GÜNTHER 1878.

Alcockia Goode et BEAN 1895.

Alexeterion VAILLANT 1888.

Aphyonus GüNTHER 1878.

Barathrites ZUGMAYER 1911.

Barathrodemus GoODE et BEAN 1886.

Barathronus Goode et BEAN 1886.

Bassobythites BRAUER 1906.

Bassogigas Goode et BEAN 1896.

Bathynectes GÜNTHER 1878.

Bathyonus GoODE et BEAN 1885.

Bathystorreus RIVERo 1934.

Bellottia Giglioli 1883.

Benthocometes Goode et BEAN 1895.

Bidenichthys BARNARD 1934.

Bothrocara BEAN 1890.

Bothrocaropsis GARMAN 1899.

Brosmophycis GILL 1861.

Brotella KauP 1858: syn. de Sirembo B. 1858.

Brotula CUVIER 1829.

Brotulina FOWLER 1946.

Brotulophis KAUP 1858.

Brotulotaenia PARR 1933.

Bythites REINHARDT 1837.

Calcarbrotula: vide Calcarobrotula F. 1946.

Calcarobrotula FOWLER 1946.

Cataety GÜNTHER 1887.

Celema Goode et Bean 1895.

Cherublemma TROTter 1926.

Cynophidium Regan 1914.

Dermatopsis OgILBy 1896.

Dermatopsoides SMITH 1948.

Dermatorus Alcock 1890.

Diancistrus OgILBY 1898. 
Dicrolene Goode et BeAN 1882. Dicromita GoODE et BEAN 1895. Dinematichthys BLEEKER 1855.

Diplacanthopoma GÜNTHER 1887. Dipulus WaITE 1905.

Enchelybrotula RADCLIFFE 1913. Eretmichthys GARMAN 1899.

Gadopsis FILIPPI 1855.

Geneiates TICKELL 1888: syn. de Brotula C. 1829.

Glyptophidium ALCocK 1889.

Grammonoides SMITH 1948.

Grimaldichthys Roule 1913 : syn. de Bassogigas G. et B. 1896.

Halias AYres 1859 : pré-employé, syn. de Brosmophycis G. 1861.

Hephthocara Alcock 1892.

Holcomycteronus GARMAN 1899 : syn. de Bassogigas G. et B. 1896.

Homostolus RADCLIFFE 1913.

Hoplobrotula GILL 1863.

Hypopleuron RADCLIFFE 1913.

Itatius Matsubara 1943.

Lamprogrammus AlCock 1891.

Leucicorus GARMAN 1899.

Leucobrotula KOEFOED 1952.

Leucochlamys ZUGMAYER 1911.

Lucifuga POEY 1860.

Luciobrotula RADCLIFFE 1913.

Lycodonus Goode et BEAN 1882.

Mastigopterus RADCLIFFE 1913.

Microbrotula GosLine 1953.

Mixonus GüNTHER 1887.

Moebia Goode et BeAN 1895.

Monomitopus Alcock 1890.

Monothrix OgILBy 1897.

Myxocephalus STEINDACHNER et DöDERLEIN 1887.

Nematobrotula GILL 1863

Nematonus GÜNTHER 1887.

Neobythites Goode et BEAN 1885.

Ogilbia Jordan et Evermann 1898.

Parabassogigas NeYBLIN 1957.

Parabrotula Zugmayer 1911.

Paradicrolene Alcock 1889.
Penopus Goode et Bean 1895.

Porogadus Goode et BFAN 1885

Poromitra Goode et BEAN 1882.

Pseudonus Garman 1899.

Pterodicromitra FOWLER 1925: s./g. de Sirembo B. 1858.

Pteroidonus GüNTHER 1887.

Pycnocraspedon Alcock 1889.

Pyramodon R.ADCliffe 1913.

Pyramodon RegaN 1914 : pré-employé.

Saccogaster ALcock 1889

Sciadonus GARMAN 1899.

Sirembo BLEEKER 1858.

Snyderidia GII.RERT 1905.

Spectrunculus JoRDAN et THOMPSON 1914.

Stygicola GILI. 1863.

Stygnobrotula BöHI.KE 1957.

Tilurella Roule 1911: larve de Brotulidae sp.

Tiluropsis Roule 1911: larve de Brotulidae sp.

Tilurus KöLlIKeR 1854: larve de Brotulidae sp.

Typhiias HuBBs 1938 : pré-employé, syn. de Typhliasina W. 1951.

Typhliasina WhItLey 1951.

Typhlonus GüNTHER 1878.

Volcanus GosLine 1954.

Watasea JORDAN et SNYDER 1901.

Xenobythites RADCLIFFE 1913.

FAM. DES RHODICHTHYIDAE

Rhodichthys COLLETT 1878.

\section{FAMILLE DES OPHIDIIDAE}

(Donzelles)

Cepolophis Kaup 1856 : syn. de Gymnelis R. 1838 : vide Zoarcidae.

Chilara Jordan et Evermann 1896.

Dannevigia WhItLey 1941.

Gengea Katayama 1949.

Genypterus PHILIPPI 1857. 
Gymnelis REINHARDT 1838 : vide Zoarcidae.

Hoplophycis KaUP 1858: s./g. de Genypterus P. 1857.

Lepophidium GILL 1895.

Leptophidium GILL 1863: pré-employé, syn. de Lepophidium G. 1895.

Ophidion LINNÉ 1758.

Ophidium : vide Ophidion L. 1758.

Otophidium GILl 1885.

Parophidion TORTONESE 1954.

Pseudophidium Gracianov 1907.

Rissola Jordan et EVERMANN 1896.

Umalius Herre et Herald 1950.

Xiphiurus Sмттн 1849: pré-employé, syn. de Genypterus P. 1857.

\section{FAMILLE DES CARAPIDAE (= Fierasferidae) (Aurins)}

Carapus RAFINESQue 1810.

Diaphasia Lowe 1843 : syn. de Carapus R. 1810.

Disparichthys HERRE 1935.

Echiodon THOMPson 1837 : syn. de Carapus R. 1810.

Encheliophiops REID 1940 : syn. d'Encheliophis M. 1843.

Encheliophis MüLLER 1843.

Fierasfer (CUVIER) OKEN 1817 : syn. de Carapus R. 1810.

Helminthodes GILL 1864.

Helminthostoma (Cocco) GüNTHER 1870 : non valide, larve de Carapus R. 1810.

Jordanicus GILBERT 1905: s./g. d'Encheliophis M. 1843.

Lefroyia JoNEs 1874 : syn. de Carapus R. 1810.

Leptofierasfer MEEK et HILDEBRAND 1928.

Onuxodon SMITH 1955.

Oxybeles RichaRdson 1844 : syn. de Carapus R. 1810.

Pirellinus WhItLEY 1928.

Porobronchus KAUP 1860: larve de Carapus R. 1810.

Rhizoiketicus VAILLANT 1893.
Schindleria Giltay 1934.

Vexillifer Gasco 1870: larve de Carapidae $\mathrm{sp}$.

Sous-ordre des AMMODYTOIDEI

FAMILLE DES AMMODYTIDAE

(Lançons ou Equilles ; Sandeels)

Ammodytes LINNÉ 1758.

Argyrotaenia Gill 1861 : syn. d'Ammodytes L. 1758.

Herklotsina FOWLER 1931

Hyperoplus GÜiNTHER 1862.

\section{FAMILLE DES BLEEKERIIDAE}

Ammodytoides DUNCKer et MOHR 1939.

Bleekeria GÜNTHER 1862.

Embolichthys JORDAN 1903.

Rhynchias GILL 1898.

FAMILLE DES HYPOPTYCHIDAE Hypoptychus STEINDACHNER 1880.

Sous-ordre des ACANTHUROIDEI

\section{FAMILLE DES ZANCLIDAE}

Gnathocentrum GUICHENOT 1866 : syn. de Zanclus (C.) C. et V. 1831.

Gonopterus Gronow 1854 : syn. de Zanclus (C.) C. et V. 1831.

Zanclus (COMMERSON) DE LACÉPÈDE 1803 : non valide.

Zanclus (C.) CUVIER et VAlencienNES 1831.

FAMILLE DES ACANTHURIDAE

$(=$ Teuthidae $=$ Hepatidae

$=$ Nasidae) (Chirurgiens)

Acanthocaulus Warte 1900 : syn. de Naso L. 1802. 
Acanthurus ForSKAL 1775.

Acronurus (GRONOW) GüNTHER 1854: larve d'Acanthurus F. 1775.

Apostasella WhitLey 1935.

Aspisurus DE LACÉPÈDE 1803: syn. d'Acanthurus F. 1775.

Atulonotus Smith 1955 : syn. de Naso L. 1802.

Axinurus CUvier 1829 : syn. d'Acanthurus F. 1775.

Burobulla WhITLEY 1931.

Callicanthus SwaInSON 1839: s./g. de Naso L. 1802.

Ceris ' vide Keris C. et V. 1835.

Colocopus GILL 1884.

Ctenochaetus GILL 1884.

Ctenodon Swainson 1839: pré-employé, syn. de Ctenochaetus G. 1884.

Ctenodon Bonaparte 1833 : non valide.

Ctenodon (S.) KLUNZINGER 1844 : pré-employé. syn. d'Acanthurus F. 1775 .

Cyphomycter FOWLER et BEAN 1929 : s./g. de Naso L. 1802.

Dasyacanthurus FOWLER 1944 : s./g. de Xesurus J. et E. 1896.

Harpurina FOWLER et BEAN 1929 : s./g. d'Harpurus F. 1778.

Harpurus FORSTER 1778.

Hepatus Gronow 1763 : non valide. Hepatus (G.) Jordan et Seale 1906 : syn. d'Harpurus F. 1778.

Keris Cuvier et VAlenciennes 1835 : syn. de Naso L. 1802.

Laephichthys OGILBY 1916.

Monoceros BLOCH et SCHNEIDER 1801 : pré-employé, syn. de Naso L. 1802.

Naseus (COMMERSON) DE LACÉPÈDE 1802 : non valide.

Naso DE LACÉPÈde 1802.

Nasonus RAFINESQUE 1815 : syn. de Naso L. 1802.

Priodon (CUviER) QuoY et GaImard 1824 : syn. de Naso L. 1802.

Priodontichthys BONAPARTE 1831: syn. de Naso L. 1802.
Prionurus DE LACÉPÈDE 1804: syn. de Naso L. 1802.

Rhinodactylus SMITH 1957.

Rhomboteuthis FOWLER 1944 : s./g. d'Acanthurus F. 1775.

Rhombotides KLEIN 1775: non valide.

Rhombotides (K.) BLEEKER 1863 : syn. d'Acanthurus F. 1775.

Scopas BONAPARTE 1831 : non valide. Scopas (B.) KNER 1865: syn. de Zebrasoma S. 1839.

Teuthis : pré-linnéen et donc non valide, syn. d'Acanthurus F. 1775.

Teuthis BROWNE 1789: non valide.

Teuthys: vide Teuthis B. 1789.

Teutis : vide Teuthis B. 1789.

Triacanthurodes FOWLER 1944.

Xesurus Jordan et Evermann 1896.

Zebrasoma SwaINSON 1839.

\section{Sous-ordre des SIG.ANOIDEI}

\section{FAMILLE DES SIGANIDAE

$$
\text { (= Teuthidae) }
$$

Amphacanthus Bloch et SCHNEIDER 1801 : syn. de Siganus F. 1775.

Buro DE LACÉPÈdE 1803: syn. de Siganus F. 1775.

Buronus RAFINESQUe 1815 : syn. de Siganus F. 1775.

Centrogaster HouTTUYN 1782: syn. de Siganus F. 1775.

Lo Jordan et Seale 1906.

Siganites FOWLER 1904.

Siganus FORSKAL 1775.

Teuthis LINNÉ 1766 : syn. de Siganus F. 1775 .

\section{Sous-ordre des BALISTOIDEI}

FAM. DES TRIACANTHODIDAE

Paratriacanthodes FOWLER 1934.

Triacanthodes BLEEKER 1858. 
Triradulifer FRASER-BRUNNER 1941: s./g. de Paratriacanthodes F. 1934.

\section{FAMILLE DES TRIACANTHIDAE}

Atrophacanthus FRASER-BRUNNER 1950.

Bathyphylax MYERS 1934.

Halimochirurgus.

Halimochirus Alcock 1899.

Hollardia POEY 1861.

Johnsonina MYERS 1934.

Macrorhamphosodes FOWLER 1934.

Marosia BEAUFORT : pré-employé, syn. de Marosichthys W. 1951.

Marosichthys WhITLEY 1951.

Parahollardia FRASER-BRUNNER 1941.

Paratriacanthodes FOWLER 1934.

Pseudotriacanthus FRASER-BRUNNER 1941.

Triacanthus (CUVIER) OKEN 1817.

Trixiphichthys FRASER-BRUNNER 1940 : s./g. de Triacanthus (C.) O. 1817.

Tydemania WEBER 1913.

\section{FAMILLE DES BALISTIDAE}

(Arbalétriers, Fanfrés)

Abalistes Jordan et Seale 1906.

Balistapus TrLesIUS 1820 .

Balistes LINNÉ 1758.

Balistoides FRASER-BRUNNER 1935.

Blandowskius WHITLEY 1931.

Canthidermis SwaINSON 1839.

Capriscus KI.EIN 1777 : non valide.

Capriscu: Röse 1793 : syn. de Balistes L. 1758.

Capriscus RAFINESQUe 1810 : syn. de Balistes L. 1758.

Capriscus Swainson 1839: syn. de Balistapus T. 1820.

Chalisoma SwaInson 1839: syn. de Balistes L. 1758.
Erythrodon RüPPELL 1852: syn. de Zenodon S. 1839.

Hemibalistes FRASER-BLUNNER 1935: s./g. de Sufflamen J. 1916.

Leiurus SwaINSON 1839: pré-employé, syn. d'Abalistes J. et S. 1906.

Melanichthys: vide Melichthys S. 1839.

Melichthys SWAINSON 1839.

Navodon WhITLEY 1930.

Nematobalistes FRASER-BRUNNER 1935.

Odonus Gister. 1848 : syn. de Zenodon S. 1839.

Oncobalistes FOWLER 1946.

Pachygnathus SwaINSON 1839: préemployé, syn. de Sufflamen J. 1916.

Pachynathus S. 1839 : errat. pro Pachygnathus S. 1839.

Parabalistes BLEEKER 1866.

Pseudobalistes BLEEKER 1866.

Pyrodon KauP 1855 : syn. de Zenodon S. 1839.

Rhinecanthus SwaINSON 1839: syn. de Balistapus T. 1820.

Scobinichthys WHITLEY 1931.

Sufflamen JoRDAN 1916.

Verrunculus JORDAN 1924 : s./g. de Balistes L. 1758.

Xanthichthys KAUP 1856.

Xenodon RüpPELL 1835 : pré-employé, syn. de Zenodon S. 1839.

Zenodon SwaINSON 1839.

\section{FAM. DES MONACANTHIDAE}

(Aluteridae) (Poissons-limes)

Acanthaluteres BLEEKER 1866.

Acreichthys FRASER-BRUNNER 1941 :

s./g. de Pervagor W. 1930.

Aleisterius: errat. pro Alutera (C.) O. 1817.

Aleuteres : errat. pro Alutera (C.) O. 1817.

Allomonacanthus FRASER-BRUNNER 1941.

Alutera (CUVIER) OKEN 1817. 
Aluterius (CUVIER) RichaRdSON 1844: vide Alutera (C.) O. 1817.

Aluterus Schinz 1822: syn. d'Alutera (C.) O. 1817.

Aluterus Cloquet 1816: syn. d'Alutera (C.) O. 1817.

Amanses GraY 1833.

Arotrolepis Fraser-BrunNer 1941.

Brachaluteres BLEEKER 1866.

Cantherines SwaINSON 1839: s./g. d'Amanses G. 1833.

Ceratacanthus GILL 1861.

Chaetodermis SwaInSON 1839.

Davidia RIBEIRO 1915.

Epimonus RAFINESQUE 1815: syn. de Monacanthus (C.) O. 1817.

Eubalichthys WHITLEY 1930.

Hanomacanthus SМाтн 1949.

Laputa Whitley 1930.

Leprogaster FRASER-BRUNNER 1941 : s./g. de Monacanthus (C.) O. 1817.

Liocornus TORTONESE 1947: s./g. de Amanses G. 1833.

Liomonacanthus BLEEKER 1866 : syn. de Cantherines S. 1839.

Meuschenia WHITLEY 1929.

Monacanthus (CUVIER) OKEN 1817.

Monoceros (PluMIER) DE LACÉPÈDE 1800 : non valide, syn. de Monacanthus (C.) O. 1817.

Nelus Whitley 1930: pré-employé, syn. de Nelusetta W. 1939.

Nelusetta WhItLey 1939.

Osbeckia JoRdAN et EVERMANN 1896.

Oxymonacanthus BLEEKER 1866.

Paraluteres BLEEKER 1866.

Paramonacanthus BLEEKER 1866.

Paramonacanthus STEINDACHNER 1867 : pré-employé,

Parika Whitley 1955.

Penicipelta WhItLey 1947.

Pervagor Whitley 1930.

Pseudalutarius BLEEKER 1865.

Pseudaluteres BLEEKER 1866: syn. de Pseudalutarius B. 1865.
Pseudaluterius : vide Pseudalutarius B. 1865 .

Pseudomonacanthus BLEEKER 1866.

Rudarius JORDAN et FOWLER 1902.

Scurrilichthys FRASER-BRUNNER 1941 : s./g. d'Arthrolepis F.-B. 1941.

Stephanolepis GILL 1861.

Tantalisor WHITLEY 1947.

Thamnoconus SMITH 1949.

Tobinia WhITLEY 1933.

Trichoderma SwaInson 1839: syn. d'Amanses Gray 1833.

Unicornis CATESBY 1771 : non valide.

\section{FAM. DES PSILOCEPHALIDAE}

Anacanthus Gray 1833: pré-employé, syn. de Psilocephalus S. 1839.

Pogonognathus BLEEKER 1849: syn. de Psilocephalus S. 1839.

Psilocephalus SWAINSON 1839.

\section{Sous-ordre des SCOMBROIDEI}

\section{FAMILLE DES SCOMBRIDAE}

(Maquereaux ; Péto ; Wahoo ; Mackrels)

Acanthocybium GILL 1862.

Acropoma OwEN : pré-employé, syn. d'Altipoma W. 1940.

Altipoma Whitley 1940.

Apodontis BennetT 1831: syn. de Scomberomorus L. 1802.

Apolectus BENNETT 1831: pré-employé, syn. de Scomberomorus L. 1802 .

Chenogaster LAHILLE 1903.

Chriomitra LockIngTON 1879: syn. de Scomberomorus L. 1802.

Cordylus Gronow 1854: syn. de Scomber L. 1758.

Cybiosarda WhITleY 1935 : s./g. de Scomberomorus L. 1802. 
Cybium Cuvier 1829 : syn. de Scomberomorus L. 1802.

Gasteroschisma RICHARDSON 1845.

Indocybium MuNRo 1943 : s./g. de Scomberomorus L. 1802.

Lepidocybium GILL 1862: syn. de Scomberomorus L. 1802.

Lepidosarda KISHINOUYE 1926.

Lepidothynnus GÜNTHER 1889.

Macrorhynchus (DE LACÉPÈDE) DUMÉRIL 1806.

Nesogrammus EvermanN et Seale 1907.

Pelamys KL.eIN 1775 : non valide.

Pneumatophorus JORDAN et GILBERT 1882.

Polipturus RAFINESQUe 1815 : syn. de Scomberomorus L. 1802.

Pseudosavara MunRo 1943: s./g. de Scomberomorus L. 1802.

Rastelliger JORDAN et STARKS 1908. Sawara JoRdan et HUBBS 1925.

Scomber LINNÉ 1758.

Scomberomorus DE LACÉPÈDE 1802. Sierra FOWLER 1905.

Wanderer WHITLEY 1937.

\section{FAMILLE DES THUNNIDAE}

(Thons ; Germons ; Bonite ;

Bonitou ; Thonine)

Albacora JORDAN 1888: syn. de Thunnus S. 1845.

Allothunnus SERVENTY 1948.

Auxis CUVIER 1829.

Creotroctes GISTEL 1848: syn. de Sarda C. 1829.

Euthynnus (LüTKEN) JORDAN et GILBERT 1882.

Germo JoRdan 1888.

Grammatorcynus GILL 1862.

Grammatorycnus GILL 1862: syn. de Grammatorcynus G. 1862.

Gymnosarda GILL 1862.

Katsuvonus KISHINOUYE 1923.

Kishinoella JoRdAN et HUBBS 1925. Megalolepis.
Neothunnus KISHINOUYE 1923.

Orcynopsis GILL 1862.

Orcynus CUVIER 1817 : pré-employé, syn. de Germo 1888.

Orycnopsis GILL 1862: vide Orcynopsis G. 1862.

Orycnus GILL 1862 : syn. de Thunnus S. 1845.

Palamita Bonaparte 1831 : syn. de Sarda C. 1829.

Parathunnus KISHINOUYE 1923.

Pelamichthys GigLIOLI 1880: syn. d'Orcynopsis G. 1862.

Pelamys Cuvier et VALENCIENNES 1831 : syn. de Sarda C. 1829.

Sarda Cuvier 1829.

Semathunnus FoWLER 1933.

Thinnus S. D. W(ood?) 1837: non valide.

Thunnus SouTH 1845.

Thynnichthy's GIG:.IOLI 1880: syn. d'Euthynnus (L.) J. et G. 1882.

Thynnus BROWNE 1789: non valide. Thynnus Cuvier 1817 : pré-employé, syn. de Thunnus S. 1845.

\section{FAMILLE DES ISTIOPHORIDAE}

(Voiliers, Marlins ou Poisson-pique)

Eumakaira Hirasaka et NaKamura 1947.

Histiophorus : emend. pro Istiophorus L. 1802.

Istiompax WHITLEY 1931.

Istipomax : errat. pro Istiompax W. 1931.

Istiophorus DE LACÉPÈDE 1802.

Kajikia HIRASAKA et NAKAMURA 1947 : syn. de Marlina G.

Lamontella SMITH 1958.

Macaria . vide Makaira L. 1803.

Machaera: vide Makaira L. 1803.

Makaira DE LACÉPÈDE 1803.

Marlina GRAY.

Marlina HIRAsaKa et NAKAMURA 1947 : pré-employé.

Notistium Herrmann 1804: syn. d'Istiophorus L. 1802.

Orthocraeros SмITH 1958. 
Pseudohistiophorus BUEN 1951. Skeponopodus NARDo 1833 : syn. de Tetrapturus R. 1810.

Tetrapturus RAFINESQUE 1810. Zanclurus SwaINSON 1839 : syn. d'Istiophorus L. 1802.

\section{FAMILLE DES XIPHIIDAE}

(Espadons, Gladiateurs ou Poisson-épée)

Phaetonichthys Nichols 1923. Xiphias LINNÉ 1758.

Sous-ordre des TRICHIUROIDEI

\section{FAMILLE DES GEMPYLIDAE}

(Rouvets, Escolars)

Acanthoderma CANTRAINE 1837 : syn. de Ruvetus C. 1833.

Acinacea BORY DE ST-VINCENT 1804 ; syn. de Gempylus C. 1829.

Aplurus Lowe 1841 : syn. de Ruvettus C. 1833.

Bipinnula JoRDAN et EVERMANN 1896: syn. d'Escolar J. et E. 1896.

Dicrotus GüNTHER 1860 (nec GuMBER): syn. de Macrorhynchus (L.) D. 1806 .

Diplogonurus NoRONHA 1926.

Diplogrammus GILL 1865.

Diretmus GüNTHER : pré-employé, syn. de Gyrionemus V. 1888.

Epinnula POEY 1854.

Escolar Jordan et Evermann 1896.

Gempylus CUVIER 1829.

Gyrionemus VAILlant 1888.

Jordanidia SNYDER 1911.

Leionura BLEEKER.

Lemnisoma LESSON 1830: syn. de Gempylus C. 1829.

Lucoscombrus VAN DER HOEVEN 1855: syn. de Gempylus C. 1829 .

Machaerope OgILby 1898.
Macrorhynchus (DE LACÉPÈDE) DUMF́RIL 1806: vide Scombridae.

Mimasea KAMOHARA 1936.

Nealotus JoHnSON 1865.

Neoepinnula Matsubara et IWAI 1952.

Nesiarchus JoHNSON 1862.

Nesogrammus.

Prometeichthys GILL 1893.

Prometheus Lowe 1841: pré-employé, syn. de Prometheichthys G. 1893.

Ptax Jordan et Evermann 1927.

Rexea WaIte 1911: syn. de Rexia W. 1911.

Rexia WaIte 1911: syn. de Jordanidia S. 1911.

Ruvettus Cocco 1833.

Scombralabrax RoU.e 1921.

Thyrsites I.ESSON 1830.

Thyrsites Cuvier 1829 : pré-employé, syn. de Leionura B.

Thyrsitoides FOWLER 1929.

Thyrsitops GILI. 1862 : syn. de Thyrsites $\mathrm{L}$.

Xenogramma.

F.AMILLE DES TRICHIURIDAE

(= Lepidopidae) (Jarretières)

Aphanopus Lowe 1839.

Assurger WhITI EY 1933.

Benthodesmus GoODE et BEAN 1882.

Diplospinus MaUI. 1948.

Enchelyopus KLEIN 1775: non valide.

En:helyopus (K.) BLEEKER 1862 : syn. de Trichiurus L. 1758.

Eupleurogrammus GILL 1862.

Evoxymetopon POEY 1863.

Gymnogaster GRONOW 1767.

Lepidopus GOUAN 1770 (nec GoNAU).

Lepturacanthus.

Lepturus (ARTEDI) GILL 1861: syn. de Trichiurus L. 1758. 
Sarcina RAFINESQUE 1810: syn. de Lepidopus G. 1770.

Symphocles RAFINESQUE 1815: syn. de Trichiurus L. 1758.

Tentoriceps WhItLEY 1950.

Trichiurus LINNÉ 1758.

Vandellia SHaw 1803: pré-employé, syn. de Lepidopus G. 1770.

Xiphotheca Montagu 1812: syn. de Lepidopus G. 1770.

Ziphotheca SwaInson 1839 : syn. de Xiphotheca M. 1812.

Zyphothyca SwaInson 1839 : syn. de Gempylus C. 1829.

\section{Sous-ordre des LUVAROIDEI}

\section{FAMILLE DES LUVARIDAE} (Louvaréou)

Ausonia Risso 1826 : syn. de Luvarus R. 1810.

Luvarus RAFINESQUE 1810.

Scrofaria Gistel 1848 : syn. de Luvarus R. 1810.

Sous-ordre des STROMATEOIDEI

FAM. DES LABRACOGLOSSIDAE

Bathystethus GILL 1893.

Cypselichthys STEINDACHNER et DöDERLEIN 1883 : syn. de Labracoglossa P. 1866.

Evistius GILL 1893.

Labracoglossa PeTERS 1866.

Platystethus GüNTHER 1860 : pré-employé, syn. de Bathystethus G. 1893.

\section{FAMILLE DES STROMATEIDAE (Fiatoles)}

Eucrotus Bean 1912.

Fiatola CUVIER 1817 : syn. de Stromateus L. 1758.
Fiatola Risso 1826: larve de Stromateus L. 1758.

Lepturus RAFINESQUE 1810: syn. de Stromateus L. 1758.

Palometa Jordan et EVERMANN 1896.

Parapsenes SmITH 1949.

Peprilus Cuvier 1829.

Poronotus GILL 1861.

Psenopsis GILL 1862.

Pterorhombus Fowler 1906.

Rhombus DE LACÉPÈDE 1800 : syn. de Peprilus C. 1829.

Seserinus (CUVIER) OKEN 1817 : syn. de Stromateus L. 1758.

Simobrama Fowler 1944

Stromateus LINNÉ 1758.

Toledia RIBEIRO 1915.

\section{FAMILLE DES PAMPIDAE}

Chondroplites GILL 1862.

Leptolepts (VAN HASSEI.T) GUICHENOT 1867: non valide.

Pampus Bonaparte 1837.

Pampus (B.) Fowler 1905 : syn. de Pampus B. 1837.

Stromateoides BLEEKER 1851: syn. de Pampus B. 1837.

\section{FAM. DES CENTROLOPHIDAE}

Acentrolophus Nardo 1827 : syn. de Centrolophus L. 1803.

Centrolophodes GILCHRIST et BONDE 1924.

Centrolophus DE LACÉPÈDE 1803.

Crius Valenciennes 1844: syn. de Schedophilus C. 1829.

Ectenias JoRDAN et THOMPSON 1914. Eurumetopos MORTON 1887: syn. d'Hyperoglyphe G. 1859.

Gymnocephalus Cocco 1838: syn. de Centrolophus L. 1803.

Hoplocoryphis GILL 1862.

Hyperoglyphe GüNTHER 1859 : s./g. de Palinurichthys B. 1859.

Icichthys JORDAN et GILBERT 1880. 
Leirus Lowe 1833 : syn. de Mupus C. 1833 .

Lirus: vide Leirus L. 1833.

Mupus Cocco 1833.

Ocycrius Jordan et HubBs 1925.

Palinurichthys BLEEKER 1859.

Palinurichthys GILL 1860: syn. de Palinurichthys B. 1859.

Palinurus DEKAY 1842: pré-employé, syn. de Palinurichthys B. 1859.

Pammelas GüNTHER 1860: syn. de Palinurichthys G. 1859.

Pompilus Lowe 1839: syn. de Centrolophus L. 1803.

Schedophilus Cocco 1829.

Tubbia WhItLeY 1943.

\section{FAMILLE DES NOMEIDAE}

$$
\text { (= Psenidae) }
$$

Ariomma JoRDAN et SNYDER 1904 : syn. de Psenes C. et V. 1833.

Atimostoma SмITH 1849: syn. de Psenes C. et V. 1833.

Bathyseriola Alcock 1890.

Caristioides WhITLEY 1950.

Cubiceps Lowe 1843 : syn. de Psenes C. et V. 1833.

Ictius JORDAN et THOMPSON 1914.

Mandelichthys NichOLS et MURPHY 1944 : s./g. de Psenes C. et V. 1833.

Mulichthys LLOYD 1909: syn. de Psenes C. et V. 1833.

Navarchus FilipPI et VeranY 1859 : syn. de Psenes C. et V. 1833.

Neptomenus GüNTHER 1860.

Nomeus CUVIER 1817.

Papyrichthys SмITH 1934: syn. de Icticus J. et T. 1914.

Paracubiceps Belloc 1937.

Psenes Cuvier et VALEnciennes 1833.

Seriolella GuICHENOT 1848.

Thecopsenes FOWLER 1944.

Trachelocirrus Doûmet 1863: syn. de Psenes C. et V. 1833.
FAM. DES TETRAGONURIDAE

Ctenodax Macleay 1886: syn. de Tetragonurus R. 1810.

Tetragonurus Risso 1810.

\section{Sous-ordre des ICOSTEOIDEI}

FAMILLE DES ICOSTEIDAE (Rag-fishes)

Icosteus LOCKINGTON 1880.

Schedophilopsis STEINDACHNER 1881 : syn. d'Icosteus L. 1880.

FAMILLE DES ACROTIDAE Acrotus Bean 1887.

Sous-ordre des ANABANTOIDEI

FAMILLE DES ANABANTIDAE (Perches grimpeuses)

Anabas CUVIer 1817.

Coius Hamilton-Buchanan 1822 : syn. d'Anabas C. 1817.

Ctenopoma Peters 1846.

Deschauensella FOWLER 1934.

Malpuletta Deraniyagala 1937.

FAM. DES OSPHRONEMIDAE

(Gouramis, Colises et Combattants)

Betta BLEEKER 1850.

Colisa Cuvier et Valenciennes 1831 : syn. de Trichogaster B. et S. 1801.

Ctenops Mc Clelland 1845: syn. d'Osphronemus L. 1801.

Lithulcus GISTEL 1848: syn. d'Osphronemus L. 1801.

Macropodus DE LACÉPÈDE 1802.

Macropus GüNTHER : emend. pro Macropodus L. 1802. 
Micracanthus SAUVAGE 1879: préemployé, syn. d'Oshimia J. 1919.

Oshimia JoRdan 1919.

Osphromenus : errat. pro Osphronemus L. 1801.

Osphronemus DE LACÉPÈDE 1801.

Parasphaerichthys PRASHAD et MUKERJI 1929.

Parophiocephalus Popta 1905 : syn. de Betta B. 1850.

Parosphromenus BLEEKER 1879.

Parosphronemus: errat. pro Parosphromenus B. 1879.

Pedites Gistel 1848 : syn. de Macropodus L. 1802.

Platypodus DE LACÉPÈdE 1804.

Platypus: vide Platypodus L. 1804.

Pseudosphromenus BLEEKER 1879.

Sphaerichthys CANESTRINI 1860.

Trichogaster BLOCH et SCHNEIDER 1801.

Trichopodus DE LACÉPÈDE 1802 : syn. d'Osphronemus L. 1801.

Trichopsis CANESTRINI 1860: syn. d'Osphronemus L. 1801.

Trichopus SHaw 1804: syn. d'Osphronemus L. 1801.

FAM. DES POLYACANTHIDAE

Belontia MYERS 1923.

Polyacanthus (KUHL) CUVIER 1829.

FAMILLE DES HELOSTOMIDAE

Helostoma (KUHL) CUVIER 1829.

Sandelia DE CASTELNAU 1861: syn. de Spirobranchus C. 1829.

Spirobranchus CUVIER 1829.

\section{FAM. DES LUCIOCEPHALIDAE}

Diplopterus GRAY 1831 : pré-employé, syn. de Luciocephalus B. 1851 .

Luciocephalus BLEEKER 1851.

\section{Sous-ordre des OPHIOCEPHALOIDEI}

FAM. DES OPHIOCEPHALIDAE

Channa Gronow 1763 : non valide. Channa (G.) Scopoli 1777: syn. d'Ophiocephalus B. 1794.

Ophicephalus BLOCH 1794.

Ophiocephalus (B.) HaMiLtoN 1822: emend. pro Ophicephalus B. 1794.

Parophiocephalus SENNA 1924 : s./g. d'Ophiocephalus B. 1794.

Philypnoides BLEEKER 1849: syn. d'Ophiocephalus B. 1794.

\section{Sous-ordre des KURTOIDEI}

FAMILLE DES KURTIDAE

Curtus : vide Kurtus B. 1786.

Cyrtus Agassiz 1846: emend. pro Kurtus B. 1786.

Kurtus BLOCH 1786.

\section{Sous-ordre des GOBIOIDEI}

FAM. DES MILYERINGIDAE

Milyeringa WHITLEY 1945.

FAM. DES RHYACICHTHYIDAE (= Platypteridae)

Platyptera (KuHL et VAN HASSELT) CUVIER 1829 : pré-employé, syn. de Rhyacichthys B. 1901.

Rhyacichthys BOULENGER 1901.

FAMILLE DES ELEOTRIDAE (incl. Gobiomoridae)

Alepideleotris HeRRE 1935. Alexurus JoRDAN 1895. Amblyeleotris BLEEKER 1874. 
Andameleotris HeRRE 1939: s./g. d'Amblyeleotris B. 1874.

Asellus (Plumier) De LACÉPÈde 1802: syn. de Gobiomorus L. 1800.

Asterropteryx RüPPell 1828.

Batanga HERRE 1946.

Batracheleotris FOWLER 1938.

Belobranchus BLEEKER 1856.

Boroda HerRe 1925.

Borodamirus WHITLEY 1935.

Bostrichthys DUMÉRIL 1806.

Bostrictis RAFINESQUE 1815 : syn. de Bostrichthys D. 1806.

Bostrychoides DE LACÉPÈdE 1802.

Bostrychus DE LACÉPÈDE 1802 : préemployé, syn. de Bostrichthys D. 1806 .

Brachyeleotris BLEEKER 1874.

Bunaka HERRE 1927.

Butis BLEEKER 1856.

Cabotia DE BUEN 1930 : pré-employé, syn. de Cabotichthys W. 1940.

Cabotichthys WHITLEY 1940.

Calleleotris GILL 1863.

Callieleotris FowLER 1934 : pré-employé, syn. de Borodamirus W. 1935.

Callogobius BLEEKER 1874: syn. de Valenciennea B. 1868.

Calumia SмIтH 1958.

Carassiops OgILby 1897.

Caulichthys OGILBy 1898 : syn. d'Hypseleotris G. 1863.

Cestreus Mc Clelland 1842.

Chriolepidops SMITH 1958.

Coryogalops SMITH 1958.

Cryptocentrops SMITH 1958.

Culius Bleeker 1856: syn. d'Eleotris B. et S. 1801.

Dactyeleotris SмIтн 1958.

Diaphoroculis FOWLER 1938.

Dormitator GILL 1861.

Eleotrica GINSBURg 1933.

Eleotriodes BLEEKER 1857: syn. de Brachyeleotris B. 1874.

Eleotrioides BLEEKER 1874.
Eleotris Bloch et SCHNEIDER 1801. Eleotris Gronow 1763 : non valide, syn. de Gobiomorus L. 1800.

Encaeura JORDAN et HUBBS 1925.

Epiphthalmus RAFINESQUE 1815 : syn. de Gobiomoroides L. 1800.

Erotelis POEY 1860.

Eviota JENKINS 1903.

Eviotops SмIтH 1957.

Fagasa Schultz 1943.

Fareleotris Sмттн 1958 : s./g. d'Amblyeleotris B. 1874.

Gergobius WhItLEY 1930: s./g. de Calleleotris G. 1863.

Gignimentum WHITLEY 1933.

Giuris SaUvage 1879.

Gobiomoroides DE LACÉPÈDE 1800.

Gobiomorphus GILL 1863.

Gobiomorus DE LACÉPÈDE 1800.

Gracieleotris HERRE 1953.

Grahamichthys WhITLEY 1956.

Guavina BLEEKER 1874.

Gymneleotris BLEEKER 1874.

Gymnobutis BLEEKER 1874.

Hemieleotris MEEK et HILDEBRAND 1916.

Hetereleotris BLEEKER 1784.

Hypseleotris GILL 1863.

Ichthyopogon: vide Ictiopogon R. 1815.

Ictiopogon RAFINESQUE 1815: syn. de Bostrichthys D. 1806.

Ioglossus BEAN 1882.

Kreffitius OgILby 1897: syn. de Mogurnda G. 1863.

Kribia Herre 1946.

Laccoeleotris FOWLER 1935.

Lairdina FOWLER 1953.

Leioeleotris FowLER 1934: syn. d'Hetereleotris B. 1874.

Lembus GüNTHer 1859 : syn. de Gobiomorus L. 1800.

Leptophilypnus MEEK et HILDEBRAND 1916.

Lindemanella WHITLEY 1935.

Lioteres SMITH 1958. 
Lizettea HERRE 1936.

Luzoneleotris HERRE 1938.

Meuschenula WhItLEY 1931.

Microeleotris MEEK et HILDEBRAND 1916.

Microphilypnus MYERS 1927.

Mogurnda GILL 1863.

Mucogobius Mc Culloch 1912 : syn de Valenciennea B. 1868.

Mulgoa OGILby 1897.

Nemateleotris FOWLER 1938.

Odonteleotris GILL 1863.

Odontobutis BLEEKER 1874.

Ophieleotris AURICH 1938.

Ophiocara GILL 1863.

Ophiorrhinus OGILBY 1897: syn. de Philypnodon B.

Oxyeleotris BLEEKER 1874.

Paloa Herre 1927.

Pariglossus : errat. pro Parioglossus R. 1912.

Parioglossus Regan 1912.

Parviparma HeRRE 1927.

Pelmatia Browne 1789 : non valide. Percottus DyBowshy 1877 : syn. de Gobiomorus L. 1800.

Philypnodon BLEEKER 1874.

Philypnus BLOCH et SCHNEIDER 1801 : syn. de Gobiomorus L. 1800.

Philypnus Cuvier et VALENCIENNES 1837 : syn. de Gobiomorus L. 1800 .

Platycephalops SMITH 1957.

Pogoneleotris BLEEKER 1875.

Pogonoculius FOWLER 1938.

Priolepis (EHRENBERG) B L E E K E R 1874: non valide, syn. d'Asterroptery $x$ R. 1828.

Prionobutis BLEEKER 1874.

Prochilus CUvier 1817 : pré-employé, syn. de Dormitator C. 1861.

Pseudolioteres SMITH 1958 : s./g. de Lioteres S. 1958.

Psiloides FISCHER 1813: syn. de Bostrychoides L. 1802.

Psilotris GINSBURg 1953.

Psilus Fischer 1813: syn. de Bostrychthys L. 1802.
Ptereleotris GILL 1863.

Pteroculiops FoWLER 1938.

Pterops RAFINESQUe 1815: syn. de Bostrychoides L. 1802.

Riukiuia FOWLER 1946.

Satulinus SмIтH 1958.

Shipwayia WHITLEY 1954.

Sineleotris SchULTZ 1943.

Tateurndia Nichols 1955.

Themistocles WHITLEY 1939: s./g. de Prionobutis B. 1874.

Typhleleotris PeTIT 1933.

Valenciennea BLEEKER 1868.

Valenciennesia BLEEKER 1868: syn. de Valenciennea B. 1868.

Vireosa JoRdAN et SNYDER 1901.

Zebreleotris HERRE 1953.

\section{FAMILLE DES GOBIIDAE}

(Gobies)

Aboma JoRdan et Starks 1895.

Abranches SMITH 1946.

Acanthogobius GILL 1859.

Acentrogobius BLEEKER 1874.

Actinogobius BLEEKER 1874.

Agunia Fowler 1946.

Ainosus Jordan et SNYDER 1901.

Alepidogobius BLEEKER 1874.

Allogobius WAITE 1904 : syn. d'Eviota J. 1903 (vide Eleotridae).

Amblychaeturichthys B L E E K E R 1874.

Amblygobius BLEEKER 1874.

Amma Ginsburg 1933: errat. pro Aruma G. 1933.

Amoya Herre 1927.

Anatirostrum ILJIN 1930.

Aparrius JORDAN et RICHARDSON 1908.

Aphyogobius WhItLEY 1931.

Apocryptes CUVIER et VALENCIENNES 1837.

Apocryptichthys DAY 1876.

Apocryptodon BLEEKER 1874. 
Apollonia ILIN 1927 : syn. de Ponticola I. 1927.

Aprolepis HuBBs 1921.

Arenigobius WHITLEY 1930.

Aruma GinsBurg 1933: s./g. de Gobiosoma G. 1859.

Asra ILJiN 1941.

Astrabe Jordan et SNyder 1901.

Atuona Herre 1935.

Aulopareia Sмгтн 1945.

Aurigobius WhitLey 1959: s./g. de Ctenogobius G. 1858.

Australaphia WhItLEY 1936.

Austrogobio OgILBy 1898.

Austrogobius DE BUEN 1951.

Austrolethops WHITLEY 1935.

Awaous STEINDACHNER 1860: syn. de Chonophorus P. 1860.

Barbatogobius Koumans 1941.

Barbulifer Evermann et EVERMANN 1888.

Bathygobius BLEEKER 1878.

Batman WhITLEY 1956.

Benthophiloides BELING et ILIN 1927.

Benthophilus EichWaLd 1831.

Berova Whitley 1928.

Biat Seale 1909.

Bikinigobius HERRE 1953.

Bollmannia JORDAN 1889.

Boreogobius GILL 1863.

Brachygobius BLEEKER 1874.

Brachyochirus NARDO 1841: préemployé, syn. de Pomatoschistus G. 1863.

Bryanina FOWLER 1932.

Bryaninops SMITH 1959.

Bubyr ILJIN 1930.

Buenia ILIN 1930.

Butigobius WhITLEY 1930.

Cabillus Sмттн 1959.

Cabotina DE BUEN 1930: pré-employé, syn. de Fagea B. 1940.

Caffrogobius SмIтt 1899.

Calamiana Herre 1945.
Caragobioides SMIтH 1945.

Caragobius Smith et Seale 1906.

Caspiosoma ILYIN 1933.

Cassigobius WHITLEY 1931.

Centrogobius BLEEKER 1874: syn. d'Oplopomus (E.) C. et V. 1837.

Cephalogobius BLEEKER 1874.

Chaenogobius GILL 1858.

Chaeturichthys RICHARDSON 1844.

Chaparrudo DE BUEN 1931.

Chasmias JORDAN et SNYDER 1901: pré-employé, syn. de Chasmichthys J. et S. 1901.

Chasmichthys JORDAN et SNYDER 1901.

Chicolepis Cockerell 1919 : syn. de Eleotriculus G. 1938.

Chlamydes JENKINS 1903.

Chlamydogobius WHITLEY 1930.

Chloea Jordan et SNYDER 1901 : préemployé, syn. de Chloeichthys W. 1940.

Chloeichthys WHITLY 1930.

Chonophorus POEY 1860.

Chriolepis GILBERT 1891.

Chromogobius DE BUEN 1931 : s./g. de Gobius L. 1758.

Cingulogobius Herre 1927 : syn. de Pleurogobius.

Clariger JORDAN et SNYDER 1901.

Clevelandia EVERMANN et EVERMANN 1888.

Congruogobius GINSBURG 1953 : s./g. de Gobionellus G. 1859.

Coronogobius HerRe 1945.

Coryphopterus GILL 1863: syn. de Rhinogobius G. 1859.

Cottogobius Koumans 1941.

Cotylopus Guichenot 1864.

Creisson Jordan et Seale 1907.

Cristatogobius HerRe 1927.

Croila Smith 1955: vide Croilia S. 1955.

Croilia SмITH 1955.

Cryptocentroides POPTA 1922.

Cryptocentrus (EHRENBERG) CUVIER et VALENCIENNES 1837. 
Cryptocentrus (E.) BLEEKER 1874 : syn. de Paragobius B.

Crystallogobius GILL 1863.

Ctenogobiops SмIтH 1959.

Ctenogobius GILL 1858.

Cyclogobius STEINDACHNER 1860 : syn. de Lepidogobius G. 1858.

Cyprinogobius Koumans 1937.

Deltentosteus GILL 1863.

Dilepidion GINSBURG 1933: s./g. de Gobiosoma G. 1859.

Doliichthys Sauvage 1874.

Doryptena SNYDER 1908.

Drombus JoRdan et SEALE 1905.

Ebomegobius HERRE 1946.

Eichwaldia SмIтT 1899 : pré-employé, syn. d'Eichwaldiella W. 1930.

Eichwaldiella WHITLY 1930.

Elacatinus JORDAN 1904.

Eleotriculus GINSBURG 1938: s./g. de Chriolepis G. 1891.

Ellerya DE CASTELnAU 1873 : syn. de Gobiodon (K. et V. H.) B. 1856.

Ellogobius WHITLE 1933.

Engrauligobius ILJIN 1930.

Enypnias Jordan et Evermann 1898.

Euctenogobius GILL 1859.

Eucyclogobius GILL 1862.

Eugnathogobius SMITH 1931.

Eutaeniichthys JORDAN et SNYDER 1901.

Evermannia JORDAN 1895.

Evermannichthys METZELAAR 1920.

Evorthodus GILL 1859.

Expedio SNYDER 1909.

Exyrias Jordan et Seale 1906.

Fagea DE BUEN 1940.

Favonigobius WhitLey 1930.

Feia SмIтн 1959.

Flabelligobius SMITH 1956.

Fluvicola ILJIN 1930.

Fusigobius WhITLEY 1930.

Fustis LIN 1932.

Galera HERRE 1927 : pré-employé, syn. de Herrea W. 1930.
Garmannia JoRDAN 1895.

Gerhardinus MEEK et HILDEBRAND 1928.

Gigantogobius FOWLER 1905.

Gillia GüNTher 1864: syn. de Gillichthys C. 1863.

Gillichthys COOPER 1863.

Gladigobius HERRE 1933.

Glossogobius GILL 1859.

Gnathogobius Sмтт 1945.

Gnatholepis BLEEKER 1874.

Gobatinus GinsBurg 1953 : s./g. de Gobionellus G. 1859.

Gobatus GinsBurg 1932: s./g. de Gobionellus G. 1859.

Gobica GinsBurg 1932: s./g. de Gobionellus G. 1859.

Gobicula GINSBURG 1945: s./g. de Garmannia J. 1895.

Gobiculina GINSBURg 1945 : s./g. de Garmannia J. 1895.

Gobidus Ginsburg 1953: s./g. de Gobionellus G. 1859.

Gobiella Sмттн 1931: syn. de Gobiopterus B. 1874.

Gobiex Ginsburg 1932: s./g. de Gobionellus G. 1859.

Gobiichthys KLUNZINGER 1871 : syn. d'Oxyurichthys B. 1858.

Gobileptes Swainson 1839.

Gobio KLEIN 1779 : non valide.

Gobiodon (KuHL et VAN Hasselt) BLEEKER 1856.

Gobiodonella LINDBERg 1934.

Gobiohelpis GINSBURG 1945: s./g. de Garmannia J. 1895.

Gobiolepis GINSBURG 1945: s./g. de Garmannia J. 1895.

Gobionellus GIRARD 1859.

Gobiopsis STEINDACHNER 1860.

Gobiopterus BLEEKER 1874.

Gobiopus GILL 1874 : syn. de Luciogobius G. 1859.

Gobiosoma GIRARD 1859.

Gobius LINNÉ 1758.

Gobulus GinSBURg 1933.

Gunnamatta WhITLEY 1928. 
Gymnogobius GILL 1863: syn. de Chaenogobius G. 1858.

Hanno Herre 1946: pré-employé, syn. de Hannoichthys H. 1950.

Hannoichthys HERRE 1950.

Hazeus Jordan et SNYDER 1901 : syn. de Gnatholepis B. 1872.

Hemigobius BLEEKER 1874.

Herrea Whitley 1930.

Herrea Sмттн 1931: pré-employé, syn. de Herreolus S. 1931.

Herreichthys Koumans : pré-employé.

Herreogobius Koumans 1940.

Herreolus SмITH 1931: syn. de Gobiopterus B. 1874.

Heteroplopomus Tоміуама 1936.

Hexacanthus NoRdMANN 1838: syn. de Benthophilus E. 1831.

Hypogymnogobius BLEEKER 1874.

Hyrcanogobius ILJIN 1928.

Iliana: vide Illana S. et S. 1906.

Iljinia DE BUEN 1930: s./g. de Pomatoschistus G. 1863.

Illana SMith et SEALE 1906.

Ilypnus Jordan et EvermanN 1896.

Innoculus WHITLEY 1952.

Intonsagobius HERRE 1945.

Inu SNYDER 1902.

Iotogobius.

Istigobius WHITLEY 1932: s./g. de Gobius L. 1758.

Itbaya HERRE 1927.

Kelloggella JORDAN et EVERMANN 1905.

Knipowitschia ILYIN 1928.

Koumansetta WHITLEY 1940.

Latrunculodes COLLETT 1875.

Latrunculus GüNTHER 1861 : pré-employé, syn. d'Aphyogobius W. 1931.

Lebetus WINTHER 1877.

Lebistes SмIтT 1899: pré-employé, syn. de Butigobius W. 1930.

Leme DevIs 1884.

Lentipes GÜNTHER 1861.

Lepidogobius GILL 1858.
Leptogobius BLEEKER 1874.

Lesueuria DUNCKER 1928: pré-employé, syn. de Lesueurigobius W. 1950.

Lesueurigobius WHITLEY 1950.

Lethops HuBBs 1926.

Leucopsarion HILGENDORF 1880.

Lizagobius WHITLEY 1933: s./g. d'Ellogobius W. 1933.

Lophiogobius GüNTHER 1873: préemployé, syn. de Cassigobius W. 1931.

Lophogobius GILL 1862.

Lubricogobius TANAKA 1915.

Lubulogobius Koumans 1940.

Luciogobius GILL 1859.

Luposicya SMTTH 1959.

Lythrypnus JORDAN et EVERMANN 1896.

Macgregorella SEALE 1909.

Macrodontogobius HERRE 1936.

Macrogobius DE BUEN 1930: s./g. de Gobius L. 1758.

Mahidolia Sмith 1932 : syn. de Waitea J. et S. 1906.

Mangarinus HERRE 1945.

Mapo Sмiтt 1899: syn. de Bathygobius B. 1878.

Mars Jordan et Seale 1906.

Mesogobius BLEEKER 1874.

Metagobius WHITLEY 1930.

Micrapocryptes HorA 1923 : syn. de Gobiopterus B. 1874.

Microgobius POEY 1875.

Micropercops F OWLER et BEAN 1920.

Microsicydium BLEEKER 1874.

Mindorogobius HERRE 1945.

Mirogobius Herre 1927: syn. de Gobiopterus B. 1874.

Mistichthys SмIтH 1902: syn. de Gobiopterus B. 1874.

Monishia SмIтн 1959.

Mugilogobius SмIтT 1899.

Mugilostoma HILDEBRAND et SCHREODER 1928 , 
Munrogobius WHTLEY 1951.

Myersina HERRe 1934.

Nematogobius BoULENGER 1910.

Neogobius.

Nes Ginsburg 1933 : s./g. de Gobiosoma G. 1859.

Nesogobius Whitley 1929.

Ninnia DE BuEN : pré-employé, syn. de Ninnigobius W. 1951.

Ninnigobius WHITLEY 1951.

Nudagobioides SHAW 1929.

Obliquogobius Koumans 1941.

Odondebuenia DE BUEN 1930.

Odonebuenia : errat. pro Odondebuenia B. 1930.

Odontogobius BLEEKER 1874.

Oligolepis BLEEKER 1874.

Ophiogobius GILL 1863.

Oplopomus SмIтH 1959: pré-employé.

Oplopomus (EHRENBERG) CUVIER et VALENCIENNES 1837.

Oplopomus STEINDACHNER 1860 : syn. de Oplopomus (E.) C. et V. 1837.

Opua JoRdAN 1925.

Oreogobius BOULENGER 1899.

Orissagobius HERRE 1945.

Orthostomus KNER 1868: pré-employé, syn. de Stomogobius W. 1931.

Ostreogobius WhITLEY 1930.

Othonops Sмгтн 1881: syn. de $\boldsymbol{T} \boldsymbol{y}$ phlogobius S. 1880.

Oxymetopon BLEEKER 1874.

Oxyurichthys BLEEKER 1858.

Padogobius BERg 1930.

Paeneapocryptes Herre 1927 : s./g. de Parapocryptes B. 1874.

Paleatogobius TAGAKI 1958.

Pallidogobius Herre 1954.

Palutrus SMITH 1959.

Pandaka Herre 1927.

Papenua Herre 1925.

Parachaeturichthys BLEEKER 1874.

Paragobiodon BLEEKER 1873.

Paragobiopsis,
Paragobius BLEEKER.

Paraphya MUNRo 1949 : pré-employé, syn. de Munrogobius W. 1951.

Parapocryptes BLEEKER 1874.

Paroxyurichthys BLEEKER 1876.

Parrella Ginsburg 1938.

Parvigobius WhITLEY 1930.

Percottus DYвowsKi 1877.

Pipidonia SмIтH 1931.

Platygobius BLEEKER 1874.

Pleurogobius.

Pleurosicya Weber 1913.

Pleurosicyops SмITH 1959.

Pogonogobius SMITH 1931.

Pomatoschistus GILL 1863.

Ponticola ILJIN 1927.

Porogobius BLEEKER 1874.

Proterorhinus SMITT 1899.

Psammichthys REGAN 1908.

Psammogobius SMITH 1935.

Pselaphias Jordan et Seale 1906.

Pseudaphya Iluin 1930.

Pseudapocryptes BLEEKER 1874.

Pseudogobiodon BLEEKER 1874.

Pseudogobiopsis Koumans 1935.

Pseudogobius.

Pseudotrypauchen HARDENBERG 1932.

Pterogobius GILL 1863.

Pycnomma RUTTER 1904.

Quietula Jordan et EVERMANN 1895.

Quisquilius JORDAN et EVERMANN 1903.

Radcliffella H и в в $\mathrm{s}$ 1921: syn. d'Evermannichthys M. 1920.

Ranulina JoRdan et Starks 1906.

Raogobius MUKERJ 1935.

Redigobius HERRE 1927.

Relictogobius PTCHELINA 1939.

Rewa WhItLEY 1951.

Rhinogobiops HUBBS 1926.

Rhinogobius GILL 1859.

Rictugobius Koumans 1932: syn. de Waitea J. et S. 1906. 
Risor GinsBurg 1933 : s./g. de Garmannia J. 1895.

Ruppellia SwaInson 1839: pré-employé, syn. de Paragobiodon B. 1873 .

Saccostoma SAUVAGE 1882 : pré-employé, syn. de Chasmichthys J. et S. 1901.

Sagamia Jordan et SNYDER 1901.

Salarigobius PFEFFER 1893.

Schismatogobius BEAUFORT 1912.

Sericagobioides HERRE 1931

Seychellea Sмrтн 1957 : syn. de Koumansetta W. 1940.

Sicya Jordan et EvermanN 1896 : pré-employé, syn. de Sicyosus J. et E. 1898 .

Sicydiops BLEEKER 1874.

Sicydium CUVIER et VALENCIENNES 1837.

Sicyogaster GILL 1860 : pré-employé, syn. de Lentipes G. 1861.

Sicyopterus GILI. 1860.

Sicyopus GILL 1864.

Sicyosus Jordan et Evermann 1898.

Silhouettea SMTTH 1959.

Sinogobius L.IN 1941: s./g. de Gobius L. 1758.

Smaragdus POEY 1860: syn. de Gobionellus G. 1859.

Smilogobius Herre 1934

Sphenentogobius FOWLER 1940.

Stenogobius BLEEKER 1874.

Stigmatogobius BLEEKER 1874.

Stiphodon WEBER 1895.

Stomogobius WhITLEY 1931.

Stupidogobius AURICH 1938.

Sufflogobius SMITH 1956.

Suruga JoRDAN et SNYDER 1901.

Synechogobius GILL 1863: syn. d'Acanthogobius G. 1859.

Syrrhothonus ChaBANAUD 1933.

Tamanka HERRE 1927.

Tasmanogobius ScotT 1935.

Thaigobiella Sмгтн 1931.

Thalassogobius HERRE 1954.
Tigrigobius FowleR 1931: s./g. de Garmannia J. 1895.

Tomiyamichthys SмIтH 1956.

Triaenapogon BLEEKER 1874.

Triaenophorichthys GILL 1858.

Triaenophorus GILL 1858: pré-employé, syn. de Triaenophorichthys G. 1858.

Trichopharynx OGII.BY 1898 : syn. de Chonophorus P. 1860.

Tridentiger GILL 1858.

Trifissus JORDAN et SNYDER 1900 : syn. de Tridentiger G. 1858.

Trimma JoRdan et SeAle 1906.

Tukogobius HERRE 1931: syn. de Rhinogobius G. 1859.

Typhlogobius STEINDACHNER 1880.

Ulcigobius FOWLER 1920.

Vailima Jordan et Seale 1906.

Vaimosa Jordan et Seale 1906 : syn. de Mugilogobius S. 1899.

Vanderhorstia SмІтн 1949.

Vitraria Jordan et EVERMANN 1903. W aitea Jordan et Seale 1906.

Waiteopsis WhITLEY 1930.

Weberogobius Koumans 1953.

Xenisthmus SNYDER 1908.

Xenogobius MetZelaAR 1920.

Yabotichthys HERRE 1945.

Yoga Whitley 1954: s./g. de Ctenogobius G. 1858.

Yongeichthys WhItLeY 1932.

Zalypnus Jordan et EVERMANN 1896.

Zebrus DE BUEN 1931: s./g. de Gobius L. 1758.

Zonogobius BLEEKER 1874.

Zostericola (ILYIN) BERG 1933 : préemployé, syn. de Zosterisessor W. 1935.

Zosterisessor WHITLEY 1935.

FAM. DES PERIOPHTHALMIDAE

(Poissons-promeneurs)

Apocryptes OSBECK 1762 : non valide. 
Apocryptes (O.) Cuvier et VAlenCIENNES 1837 : syn. de Beleophthalmus C. et V. 1837.

Boleophthalmus CUVIER et VALENCIENNES 1837.

Boleops GILl 1863 : syn. de Scartelaos S. 1839.

Euchoristopus GILL 1863.

Gobilepres (SwaInson) B L E E K E R B. et S. 1801 .

Periophthalmodon BLEEKER 1874 : syn. de Periophtalmus B. et S. 1801.

Periophthalmus BLOCH et SCHNEIDER 1801.

Scartelaos SwaInSON 1839.

\section{FAMILLE DES KRAEMERIIDAE}

Kraemeria STEINDACHNER 1906.

Psammichthys de Kraemeria S. 1906.

: syn.

Vitreola Jordan et SeAle 1906 : syn. de Kraemeria S. 1906.

\section{FAMILLE DES GOBIOIDIDAE}

(incl. Paragobioididae)

Cayennia Sauvage 1880: syn. de Gobioides L. 1800.

Gobioides DE LACÉPÈDE 1800.

Ognichodes SwaInson 1839: syn. de Gobioides L. 1800.

Paragobioides KendALl et GoldsBOROUGH 1911.

Paratyntlastes GILTAY 1925 : syn. de Gobioides L. 1800.

Plecopodus RAFINESQUE 1815: syn. de Gobioides L. 1800.

Psilosomus SWAINSON 1839.

Tyntlastes GüNTHER 1862: s./g. de Gobioides L. 1800.

\section{FAMILLE DES TAENIOIDIDAE}

Amblyopus Cuvier et VALEnciennes 1837: syn, de Taenioides L. 1800 ,
Brachyamblyopus BLEEKER 1874.

Gymnurus RAFINESQUE 1815: syn. de Taenioides L. 1800.

Pteropterus SwaInson 1839: syn. de Taenioides L. 1800.

Odontamblyopus BLEEKER 1874.

Taenioides DE LACÉPÈDE 1800.

\section{FAM. DES TRYPAUCHENIDAE}

Amblyotrypauchen HoRA 1924.

Ctenotrypauchen STEINDACHNER

1867: syn. de Trypauchenichthys B. 1860.

Trypauchen CUVIER et VALENCIENNES 1837.

Trypauchenichthys BLEEKER 1860 . Trypauchenophrys FRANZ 1910.

Trypauchenopsis VoLz 1903.

Sous-ordre des BATRACHOIDEI

\section{FAM. DES BATRACHOIDIDAE}

(Poissons-crapauds, Poissons-chanteurs)

Amphichthys SWAINSON 1839.

Austrobatrachus SМITH 1949.

Barchatus SмITH 1952.

Bathybatrachus GILCHRIST et BONDE 1924.

Batrachoides DE LACÉPÈDE 1800.

Batrachomoeus OGILBy 1908.

Batrachus BLOCH et SCHNEIDER 1801.

Batrichthys SмIтH 1935.

Batrictius RAFINESQUe 1815 : syn. de Batrachoides L. 1800.

Chatrabus SмIтн 1949.

Coryzichthys OGILBY 1908.

Daector Jordan et EVERMANN 1898.

Gymnobatrachus SMITH 1949.

Halobatrachus OGILBY 1908.

Halophryne GILL 1863.

Marcgravia JoRDAN 1886 : syn. d'Am. phichthys S. 1839. 
Marcgravichthys RIBEIRO 1915 : syn. d'Aphichthys S. 1839.

Nautopaedium JoRDAN 1919.

Opsanus RAFINESQUe 1818.

Phrynotitan GILL 1885.

Porichthys GIRARD 1854.

Pseudobatrachus DE CASTELNAU 1875: pré-employé, syn. de Batrachomoeus O. 1908.

Riekertia SмIтн 1952.

Sanopus SмITH 1952.

Thalassophryne GüNTHER 1861.

Thalassothia BERG 1895.

Tharbacus SMITH 1952.

Triathalassothia FOWLER 1944.

Sous-ordre des GOBIESOXOIDEI

FAMILLE DES GOBIESOCIDAE (Porte-écuelle, Barbiers, Ventouseurs) Acyrtops Schultz 1951.

Acyrtus Schultz 1944 : syn. d'Arcos S. 1944.

Apepton Gistel 1848 : syn. de Gouania N. 1832.

Aphos HubBs et Schultz 1939.

Apletodon Briggs 1955.

Arbaciosa Jordan et Evermann 1896.

Arcos Schultz 1944.

Aspasma Jordan et Fowler 1902.

Aspasmogaster WAITE 1907.

Aspasmichthys BRIGGS 1955.

Aspasmodes SMITH 1958.

Bryssetaeres JORDAN et EVERMANN 1896.

Bryssophilus JORDAN et EVERMANN 1898.

Caularchus GILL 1862.

Caulistius JORDAN et EVERMANN 1896.

Chorisochismus BRISOUT DE BARNEVILLE 1846.

Cochlioceps WhItLEY 1943.
Conidens Briggs 1955.

Coronichthys Herre 1943: syn. de Diademichthys P. 1942.

Cotylichthys JORDAN 1919.

Cotylis Müller 1843: syn. de Gobiesox L. 1800.

Cotylis GüNTHER : pré-employé, syn. de Cotylichthys J. 1919.

Creocele Briggs 1955.

Crepidogaster GüNTHER 1861: préemployé, syn. d'Aspasmogaster W. 1907.

Dellichthys BRIGgs 1955.

Diademichthys PfafF 1942.

Diplecogaster FRASER-BRUNNER 1938.

Diplocrepis GÜNTHER 1861.

Eckloniaichthys Sмгтн 1943.

Gastrocyathus BRIGGS 1955.

Gastrocymba BRIGgS 1955.

Gastroscyphus BRIGgS 1955.

Gobiesox DE LACÉPÈDE 1800.

Gouania NARDO 1832.

Haplocylix BRIGGS 1955.

Infratridens Schultz 1944.

Lecanogaster BRIGGS 1957.

Lepadichthys WAITE 1904.

Lepadogaster GOUAN 1770.

Leptopterygius TrosCHEL 1860 : syn. de Gouania N. 1832.

Liobranchia BRIGGS 1955.

Megaphalus RAFINESQUE 1815: syn. de Gobiesox L. 1800.

Mirbelia CANESTRINI 1864 : s./g. de Lepadogaster G. 1770.

Oliverichthus WHITLEY et PHILLIPPS 1939.

Opeatogenys BRIGGs 1955.

Parvicrepis Whitley 1931.

Pherallodiscus Briggs 1955.

Pherallodus BRIgGs 1955.

Piescephalus RAFINESQUE 1810 : syn. de Lepadogaster G. 1770.

Rimicola JoRDAN et EVERMANN 1896: s./g. de Gobiesox L. 1800.

Rupisuga SwaINSON 1839: syn. de Gouania N. 1832. 
Sicyases MüLler 1843.

Sicyogaster BRISOUT DE BARNEVILLE 1846:

Tomicodon BRISOUT DE BARNEVILLE 1846 : syn. de Sicyases M. 1843.

Tracheloschismus BRISOUT DE BARNEVILLE 1846.

Volgiolus WhItLey 1931: s./g. de Parvicrepis W. 1931.

\section{Sous-ordre des ECHENEOIDEI}

\section{FAMILLE DES ECHENEIDAE}

(Rémoras ou Sucets)

Echeneis LINNÉ 1758.

Leptecheneis GILL 1864: syn. d'Echeneis L. 1758.

Phtheirichthys GILL 1862.

Remilegia GILL 1862.

Remora CATESBY 1771: non valide.

Remora FORSTER 1771.

Remora Gill 1862 : syn. de Remora F. 1771 .

Remorina JORDAN et EVERMANN 1896.

Remoropsis GILl 1863: s./g. de Remora F. 1771.

Rhombochirus GILL 1863.

Sous-ordre des SCORPAENOIDEI

FAMILLE DES SCORPAENIDAE (Rascasses)

Abcichthys WhITLEY 1927.

Acutomentum Evermann et BeEson 1893: s./g. de Sebastes C. 1829.

Acutospina Evermann et Beeson 1893.

Adventor Whitley 1952: s./g. de Membracidichthys.

Allosebastes HunBs 1951: s./g. de Sebastodes G. 1861.
Amblyapistus BLEEKER 1876. Apistes Cuvier 1829: pré-employé, syn. de Pterichthys S. 1839.

Apistus: vide Apistes C. 1829.

Bathysebastes STEINDACHNER 1885. Brachirus Swainson 1839: syn. de

Dendrochirus S. 1839.

Brachypterois FOWLER 1938.

Brachyrus SwaInSON 1839: syn. de Dendrochirus S. 1839.

Centropogon GüNTHER 1860.

Coccotropsis BARNARD 1927.

Cocotropus KaUP 1858.

Corythobatus CANTOR 1849.

Cottapistus BLEEKER 1876.

Crossoscorpaena FOWLER 1938.

Daia OgILby 1904.

Decterias JoRdan et StaRKs 1904.

Dendrochirus SWAINSON 1839.

Dendroscorpaena SMITH 1957.

Ebosia Jordan et Starks 1904.

Ectreposebastes GARMAN 1899.

Emmelas Jordan et EVERMANN 1898.

Eosebastes JORDAN et EVERMANN 1896.

Erisphex JORDAN et STARKS 1904.

Eusebastes Sauvage 1878: syn. de Sebastes C. 1829.

Gadapistus BEAUfort 1949 : syn. de Neocentropogon M. 1943.

Glyptauchen GüNTHER 1860.

Gymnapistes SWAINSON 1839.

Hatumeus MATSUBARA 1943: s./g. de Sebastes C. 1829.

Helicolenus Goode et BeAn 1895.

Hipposcorpaena FOWLER 1938.

Hispanicus JoRDAN et EVERMANN 1896.

Holoscorpaena FOWLER 1944.

Hoplosebastes SCHMIDT 1929.

Hozukius Matsubara 1934.

Hypodytes Gistel 1848: syn. de Pterichthys S. 1839.

Hypomacrus EVERMAN et SEALE 1907. 
Iracundus JORDAN et EVERMANN 1903.

Kanekonia TANAKA 1915.

Kantapus Sмттн 1946: syn. de Sabastapistes G. 1878 .

Liocranium OGILbY 1904.

Lioscorpius GüNTHER 1880.

Lythrichthys JORDAN et STARKS 1904.

Macrochyrus SwaINSon 1839: syn. de Pterois (C.) O. 1817.

Macroscorpius FOWLER 1938.

Maxillicosta WHITLEY 1935.

Mebarus Matsubara 1950 : s./g. de Sebastes C. 1829.

Merinthe SNYDER 1904.

Metzelaaria JORDAN 1923.

Murasoius MATSUBARA 1943: s./g. de Sebastes C. 1829.

Nemapontinus FOWLER 1938.

Nemapterois Fowler 1938.

Neocentropogon Matsubara 1943.

Neohispanicus MATSUBARA 1943 : s./g. de Sebastes C. 1829.

Neomerinthe FoWLER 1935.

Neosebastes GUICHENOT 1867.

Notesthes OgILby 1904.

Oligoscorpaena FOWLER 1939.

Osorisa de Scorpaena L. 1758.

Panotus RAFINESQUe 1815: syn. de Pterichthys S. 1839.

Parabrachirus MATSUBARA 1943 : syn. de Parapterois B. 1876.

Paracentropogon BLEEKER 1876 : syn. de Pterichthys S. 1839.

Paraminous FowLER 1943.

Parapterois BLEEKER 1876.

Parascorpaena BLEEKER 1876.

Parascorpaenodes SMITH 1957.

Parocosia Whitley 1958: s./g. d'Amblyapistus B. 1876.

Paronescodes SMITH 1958.

Pentaroge Günther 1860: syn. de Gymnapistes S. 1839.

Peristrominous WhITLEY 1952.

Phenacoscorpius FOWLER 1938.
Platypterus SwaInson 1839 : pré-employé, syn. de Tetraroge G. 1860.

Plectrogenium GILBERT 1905.

Pogonoscorpius Regan 1908.

Polemius Kaup 1858: syn. de Petrichthys S. 1839.

Pontinus POEY 1860.

Primospina Evermann et BeEson 1893.

Prosopodasys CANTOR 1849 : syn. de Vespicula J. et R. 1910.

Pseudopterus KLEIN 1775 : non valide.

Pseudopetrus (K.) BLEEKER 1876 : syn. de Pterois (C.) O. 1817.

Pseudosebastes Sauvage 1878.

Ptarmus SMITH 1946.

Pterichthyodes BLEEKER 1859.

Pterichthys SwaINSON 1839.

Pteroidichthys BLEEKER 1856.

Pteroidichthys BLEEKER 1876.

Pterois (CUVIER) OKEN 1817.

Pteroleptus Swainson 1839: syn. de Pterois (C.) O. 1817.

Pteropelor FowLer 1938.

Pteropodus Evermann et Beeson 1893.

Pteropterus SwaInson 1839: syn. de Pterois (C.) O. 1817.

Ranipterois WHITLEY 1952.

Rhabdosebastes FOWLER et BEAN 1922.

Richardsonichthys SмIтH 1958.

Rosicola JoRDAN et EVERMANN 1896.

Ruboralga WHITLE 1931.

Scorpaena LINNÉ 1758.

Scorpaenella FowLER 1938.

Scorpaenichthys GIRARD 1854.

Scorpaenichthys BLEEKER 1856 : préemployé, syn. de Scorpaenopsis H. 1837.

Scorpaenodes BLEEKER 1857.

Scorpaenopsella FOWLER 1938.

Scorpaenopsis HeCKEL 1837.

Scorpius (Plumier) DE LACÉPÈde 1802 : non valide. 
Sebastapistes GILL 1878.

Sebastavus JoRdAn et GILbERT 1919.

Sebastella TANAKA 1918.

Sebastes CUVIER 1829.

Sebastichthys GILL 1862.

Sebastinus JoRdAN 1920.

Sebastiscus Jordan et Starks 1904.

Sebastocarus JORDAN et EVERMANN 1927.

Sebastocles Jordan et HubBs 1925 : s./g. de Sebastes C. 1829.

Sebastoessus JORDAN 1920.

Sebastaessus JORDAN 1920.

Sebastodes GILL 1861.

Sebastolobus GILL 1881.

Sebastomus GILL 1864.

Sebastoplus GILL 1863.

Sebastopsis GILL 1862: syn. de Scorpaenodes B. 1857.

Sebastopsis Sauvage 1873: syn. de Scorpaenodes B. 1857.

Sebastopyr JORDAN et EVERMANN 1927.

Sebastosemus GILL 1905.

Sebastosomus GILL 1863.

Setarches JOHNSON 1863.

Sibogapistus BEAUFORT 1949.

Taenionotus DE LACÉPÈDE.

Takenokius MATSUBARA 1943 : s./g. de Sebastes C. 1829.

Tetraroge GüNTHER 1860.

Thysanichthys JORDAN et STARKS 1904.

Trachyscorpia GINSBURG 1953.

Trichophasia SwaInSON 1839: syn. de Vespicula J. et R. 1910.

Trichosomus SwaINSON 1839: syn. de Vespicula J. et R. 1910.

Vadesuma WhItLeY 1933.

Vespicula JORDAN et RICHARDSON 1910.

\section{FAMILLE DES TRIGLIDAE (Grondins)}

Aoyagichthys Whitley 1958: s./g. de Paratrigla O. 1911.
Aspitrigla Fowler 1925.

Bellator JORDAN et EVERMANN 1896 : pré-employé, syn. de Vexillitrigla W. 1931.

Bovitrigla FOWLER 1939.

Chelidonichthys Kaup 1873 : syn. de Trigla L. 1758.

Chriolax JoRDAN et GILBERT 1878 : syn. de Prionotus L. 1802.

Colotrigla GILL 1905.

Corystion KLEIN 1775 : non valide.

Currupiscis WhITLEY 1931.

Dinichthys KaUP 1872 : pré-employé, syn. de Marubecula W. 1958.

Dixiphichthys FOWLER 1938.

Dixiphistes FOWLER 1938.

Dixiphistops FOWLER 1938.

Eutrigla Fraser-BRUNNer 1938.

Exolissus JORDAN 1923.

Fissala GILL 1905.

Gurnardus JORDAN et EVERMANN 1898.

Hatha WhITLEY 1959.

Hoplonotus GUICHENOT 1866: préemployé, syn. de Petrygotrigla W. 1899.

Lepidotrigla GÜNTHER 1860.

Lyrichthys KauP 1873 : syn. de Trigla L. 1758.

Marubecula WHITLEY 1950.

Merulinus JoRdAn et EverMANN 1898: pré-employé, syn. de Triscurrichthys W. 1931.

Mierotrigla KAUP 1873.

Ornichthys SwaINSON 1839: syn. de Prionotus L. 1802.

Otohime JORDAN et STARKS 1907 : syn. de Pterygotrigla W. 1899.

Pachytrigla FOWLER 1938.

Palaenichthys KAUP 1873: syn. de Lepidotrigla G. 1860.

Paraheminodus Kamohara 1957.

Parapterygotrigla MATSUBARA 1937.

Paratrigla OgILby 1911.

Prionotus DE LACÉPÈDE 1802.

Pristhoplotrigla FOWLER 1938 : s./g. de Lepidotrigla G. 1860. 
Pterygotrigla WaITE 1899.

Satyrichthys de Lepidotrigla G. 1860.

Stagonotrigla FOWLER 1938.

Trigla LINNÉ 1758.

Trigloporus SмIтH 1934: s./g. de Trigla L. 1758.

Triscurrichthys WHITLEY 1931.

Uradia KamOHARA 1938.

Vexillitrigla WhItLeY 1931.

\section{FAMILLE DES PERISTEDIIDAE (Malarmats)}

Acanthistedion FOWLER 1943.

Gargariscus SМाтн 1917.

Heminodus SмІтн 1917.

Nemaperistedion FOWLER 1938.

Octonus RAFINESQUe 1810: syn. de Peristedion L. 1802.

Panichthys Whitley 1933: s./g. de Peristedion L. 1802.

Peristedion DE LACÉPÈDE 1802.

Peristethidium de Peristedion L. 1802.

Peristethion: vide Peristedion L. 1802.

Peristethus Kaup 1858 : syn. de $\boldsymbol{P e}$ ristedion L. 1802.

Scalicus JoRdAN 1922.

Vulsiculus JORDAN et EVERMANN 1896.

\section{FAM. DES CARACANTHIDAE}

Amphiprionichthys BLEEKER 1855 : syn. de Caracanthus K. 1845.

Caracanthus KRÖYER 1845.

Centropus KNER 1860: pré-employé, syn. de Caracanthus K. 1845.

Crossoderma Guichenot 1869.

Micropus Gray 1831 : pré-employé, syn. de Caracanthus K. 1845.

Trachycephalus DE VIS 1884: préemployé, syn. de Caracanthus K. 1845 .
FAMILLE DES SYNANCEJIDAE

Bufichthys Swainson 1839.

Choridactylodes GILCHRIST 1902.

Choridactylus RICHARDSON 1848.

Chorismodactylus GüNTHER 1860: errat. pro Choridactylus R. 1848.

Chorismopelor CHEvey 1927.

Corythobatus CANTOR 1849: syn. de Minous C. et V. 1828.

Dampierosa WhItLeY 1932.

Deleastes Seale 1906.

Emmydrichthys JORDAN et RUTTER 1896 : syn. de Synanceja B. et S. 1803.

Erosa SWAINSON 1839.

Erosa (S.) Jordan et Starks 1904.

Glyptauchen GÜNTHER 1860.

Inimicus JORDAN et STARKS 1904: syn. de Pelor C. 1829.

Leptosynanceia BLEEKER 1874.

Lysodermus SMITH et POPE 1906.

Minous CUVIER et VALENCIENNES 1829.

Nofua Whitley 1930 : s./g. de Synanceja B. et S. 1803.

Pelor Cuvier 1829.

Peloropsis GILbert 1905.

Polycaulus GüNTHer 1860.

Pseudosynanceia DAY 1875.

Rhinopias GILL 1905.

Simopias GILL 1905.

Spurco (Commerson) Cuvier et VALENCIENNES 1829 : non valide.

Synanceia : vide Synanceja B. et S. 1803.

Synanceichthys BLEEKER 1863: syn. de Synanceja B. et S. 1803.

Synanceja BLOCH et SCHNEIDER 1803.

Synanchia Swainson 1839: syn. de Synanceja B. et S. 1803.

Synancidium Müller 1843: syn. de Synanceja B. et S. 1803.

Trachicephalus SWaINSON 1839: préemployé, syn. de Polycaulus G. 1860. 
Uranoblepus Gill 1861 : syn. de Polycaulus G. 1860.

\section{FAMILLE DES PATAECIDAE}

Neopataecus STEINDACHNER 1884.

Pataecus RICHARDSON 1844.

\section{FAM. DES GNATHACANTHIDAE}

Beridia de CASTelnau 1877 : syn. de Gnathacanthus B. 1855.

Gnathacanthus BLEEKER 1855.

Holoxenus GüNTHER 1876: syn. de Gnathacanthus B. 1855.

\section{FAM. DES HEXAGRAMMIDAE}

Acantholebius GILL 1861 : syn. d'Hexagrammos (S.) T. 1809.

Agrammus GüNTHER 1860.

Chiropsis GIRARD 1857 : syn. d'Hexagrammos (S.) T. 1809.

Chirus (Steller) Pallas 1811: syn. d'Hexagrammos (S.) T. 1809.

Decagrammus HuBBs 1928.

Grammatopleurus GILL 1861 : syn. d'Hexagrammos (S.) T. 1809.

Hexagrammoides Gracianov 1907 : syn. d'Hexagrammos (S.) T. 1809.

Hexagrammos (STEller) Tilesius 1809.

Hexagrammus : vide Hexagrammos (S.) T. 1809.

Labrax Pallas 1810 : pré-employé, syn. d'Hexagrammos (S.) T. 1809.

Lebius (Steller) Pallas 1811 : syn. d'Hexagrammos (S.) T. 1809.

Octogrammus BLEEKER 1874: syn. d'Hexagrammos (S.) T. 1809.

Pleurogrammus GILL 1861.

Stellistius JoRdan et TANAKA 1927.

Xanthocles JoRDAN 1917.
FAM. DES ANOPLOPOMATIDAE

$$
\text { (= Anoplomidae) }
$$

Anoplopoma AYRES 1858.

Scombrocottus PeTers 1872: syn. d'Anoplopoma A. 1858.

\section{FAMILLE DES ERILEPIDAE}

Ebisus JORDAN et SNYDER 1901 : syn. d'Erilepis G. 1893.

Erilepis GILL 1893.

Myriolepis LocKINGTON 1880 : préemployé, syn. d'Erilepis G. 1893.

FAMILLE DES OPHIODONTIDAE Ophiodon GIRARD 1854.

Oplopoma G I R A R D 1856: syn. d'Ophiodon G. 1854.

FAMILLE DES OXYLEBIIDAE

Oxylebius GILL 1862.

FAMILLE DES ZANIOLEPIDAE

Xanthocles JORDAN 1917.

Zaniodermis GüNTHER 1860: errat. pro Zaniolepis G. 1857.

Zaniolepis GIRARD 1857.

FAM. DES PLATYCEPHALIDAE Amora Gray 1833: serait syn. de Thysanophrys O. 1898.

Anoara GRAY 1833: errat. pro Amora.

Bambradon JORDAN et RICHARDSON 1908.

Bembradium GILBERT 1905.

Bembras CuVier et VALENCIENNES 1829.

Brachybembras FOWLER 1938.

Cacumen WhITLEY 1931.

Calliomorus DE LACÉPÈDE 1800 : syn. de Platycephalus B. 1795.

Centranodon DE LACÉPÈDE 1803 : syn. de Platycephalus B. 1795. 
Cocciella Whitley 1940.

Coccius Jordan et HubBs 1925 : préemployé, syn. de Cocciella W. 1940.

Colefaxia WhItLEY 1935: s./g. de Neoplatycephalus C. 1872.

Cumbel WhitLey 1952.

Cymbacephalus FOWLER 1938.

Elates Jordan et SEALE 1907.

Grammoplites FOWLER 1904.

Hyalorhynchus OGILBY 1910: syn. d'Elates J. et S. 1907.

Inegocia JORDAN et THOMPSON 1913.

Insidiator JORDAN et SNYDER 1900 : pré-employé, syn. de Suggrundus W. 1930.

Kumococius Matsuraba et OchiaI 1955.

Levanaora WHITLEY 1933.

Leviprora WHITLEY 1931.

Longitrudis WHITLEY 1931.

Neoplatycephalus DE CASTELNAU 1872.

Onigocia JoRDAN et THOMPSON 1913.

Papilloculiceps Fowler et STEINITZ 1956.

Parabembras BleEKer 1874.

Planiprora WhItLey 1931.

Platycephalus BLOCH 1795.

Ratabulus JoRDAN et HUBBS 1925.

Repotrudis WhItLEY 1930: s./g. de Suggrundus W. 1930.

Rogadius JORDAN et RICHARDSON 1908.

Sorsogona HerRe 1934.

Suggrundus WhitLeY 1930.

Thysanophrys OgILBy 1898.

Trudis WhITLEY 1931.

Wakiyus JoRdAN et HuBBs 1925.

\section{FAMILLE DES APLOACTIDAE}

Acanthosphex FOWLER 1938.

Aniculerosa Whitley 1933.

Aploactis Temminck et Schlegel 1843.
Aploactisoma dE CASTelnaU 1872: syn. d'Aploactis T. et S. 1843.

Aploactoides Fowler 1938.

Cocotropus KaUP 1858.

Insopiscis WHITLEY 1933.

Membracidichthys WHITLEY 1933.

Paraploactis BLEEKER 1865.

Sthenopus RICHARDSON 1848: préemployé, syn. de Trichopleura K. 1858 .

Trichopleura KAUP 1858.

FAM. DES BATHYAPLOACTIDAE

Bathaploactis: vide Bathyaploactis W. 1933.

Bathyaploactis WHITLEY 1933.

Kleiwegia BEAUFORT 1952.

FAMILLE DES CONGIOPODIDAE

$$
\text { (= Agriopidae) }
$$

Agriopus Cuvier 1829: syn. de Congiopus P. 1811.

Cephalinus Gronow 1854: syn. de Congiopus P. 1811.

Congiopodus Regan 1913 : vide Congiopus P. 1811.

Congiopus PERRY 1811.

Ocosia Jordan et STARKS 1904.

Perryena WhItLeY 1940.

Snyderina Jordan et Starks 1901.

Zanclorhynchus GüNTHER 1880.

\section{FAM. DES HOPLICHTHYIDAE}

Acanthoplichthys FOWLER 1943.

Hoplichthys CUVIER et VALENCIENNES 1829.

Monhoplichthys FOWLER 1938.

Oplichthys: vide Hoplichthys C. et V. 1829.

Pristhoplichthys FOWLER 1938.

Rhinoplichthys FOWLER 1938. 


\section{Sous-ordre des COTTOIDEI}

\section{FAMILLE DES JORDANIIDAE}

Alcidea Jordan et Evermann 1898.

Jordania STARKS 1895.

Paricelinus Evermann et EvermanN 1889.

\section{FAMILLE DES ICELIDAE}

Agonocottus Pavlenko 1910.

Allartedius HubBs 1926: syn. d'A $\boldsymbol{r}$ tediellus J. 1885.

Alloricuzenius Matsubara et Iwal 1951 : s./g. de Ricuzenius J. et S. 1904.

Archaulus GILBERT et BURKe 1912. Archistes Jordan et GILBERT 1898. Artedielloides Soldatov 1923: syn. d'Artediellus J. 1885.

Artediellus JORDAN 1885.

Artedius GIRARD 1856.

Aspicottus GIRARD 1854.

Astrocottus Bolin 1936.

Astrolytes Jordan et Starks 1895. Atopocottus Bolin 1936.

Axyrias StARKs 1896.

Calycilepidotus AYRES 1855.

Ceratocottus GILL 1859.

Chitonocottus LOCKINGTON 1881.

Clypeocottus Ayres 1854 : syn. d'Aspicottus G. 1854.

Daruma Jordan et StARKs 1904.

Enophrys SwaINSON 1839.

Hemilepidotus CUVIER 1829.

Icelichthys SснміDT 1935: s./g. d'Icelus K. 1845.

Icelinus JORDAN 1885.

Icelus KRÖYER 1845.

Medicelinus BoLIN 1935: s./g. d'Icelinus J. 1885.

Melletes BEAN 1879.

Noviricuzenius BoLIN 1936: s./g. de Ricuzenius J. et S. 1904.

Ochotskia Schмidт 1923.
Orthopnias Starks et MANn 1911. Parartedius HubBs 1926.

Parastrolytes HubBs 1926.

Penicelinus Bolin 1936 : s./g. d'Icelinus J. 1885.

Prionistius BeAN 1883.

Pterygiocottus BeAn et Weed 1920.

Radulinus GILBERT 1890.

Rastrinus J O R D A N et EVERMANN 1896.

Ricuzenius JoRdan et STARKS 1904.

Ruscariops HUBBS 1926.

Ruscarius JORDAN et STARKS 1895.

Schmidtia Jordan et Starks 1904: pré-employé, syn. de Schmidtina J. et S. 1904.

Schmidtina Jordan et STARKS 1904.

Stelgidonotus JoRDAN et THOMPSON 1905.

Stelgistrops HubBs 1926.

Stelgistrum JORDAN et GILBERT 1898.

Sternias Jordan et EVERMANN 1898. Stlengis Jordan et StarKs 1904.

Tarandichthys JORDAN et EVERMANN 1896.

Temnistia RICHARDSON 1836: syn. d'Hemilepidotus C. 1829.

Thyriscus GILBERT et BURKE 1912. Triglops REINHARDT 1832.

FAMILLE DES BLEPISIIDAE

Blepsias CUVIER 1829.

Histiocottus GILL 1888: syn. de Blepsias C. 1829.

Nautichthys GIRARD 1859.

Nautiscus Jordan et EVERMANN 1898.

Peropus Lay et BenNetT 1839 : préemployé, syn. de Blepsias C. 1829.

\section{FAMILLE DES}

SCORPAENICHTHYIDAE

Scorpaenichthys GIRARD 1854. 


\section{FAMILLE DES COTTIDAE}

\section{(Chabots)}

Acanthocottus GIRARD 1851 : syn. de Myoxocephalus (S.) T. 1811.

Ainocottus Jordan et STARKS 1904. Alcichthys JORDAN et STARKS 1904. Allocottus HubBs 1926.

Antipodocottus BoLIN 1952.

Aphobus Gistel 1848 : syn. de Cen-

tridermichthys R. 1844.

Argyrocottus Herzenstein 1892.

Bero JoRdAN et STARKS 1904.

Blennicottus GILL 1861.

Boreocottus GILl 1859: syn. de Myoxocephalus (S.) T. 1811.

Bunocottus KNER 1868 (serait un Scorpaenidae fide JORDAN 1923).

Centridermichthys RICHARDSON 1844

Cephalocottus Gracianow 1907.

Clinocottus GILL 1861.

Cottiusculus SchMIDT 1904.

Cottopsis GIRARD 1851.

Cottunculoides BARNARD 1927.

Cottunculus COLLetT 1875.

Cottus LINNÉ 1758.

Crossias JoRdan et STARKS 1904: syn. de Prococottus G. 1859.

Dasycottus BEAN 1890.

Dialarchus GREeLEY 1901.

Ebinania SAKAMOTO 1932.

Elaphichthys JORDAN et STARKS 1904.

Elaphocottus Sauvage 1878: syn. de Gymnecanthus S. 1839.

Evermanniana TARANETZ 1935.

Eximia GREELEY 1901 : pré-employé, syn. de Greeleya J. 1920.

Furcina Jordan et Starks 1904.

Greeleya JORDAN 1920.

Gymnacanthus SwaINSON 1839 : vide Gymnocanthus S. 1839.

Gymnocanthus SWAINSON 1839.

Hoplocottus KaUP 1858: syn. de Pseudoblennius T, et S. 1850.
Leiocottus GIRARD 1856

Leocottus TALIEV 1958: s./g. de Paracottus.

Leptocottus GIRARD 1854.

Malacocottus BEAN 1890.

Megalocottus GILL 1861: syn. de Myoxocephalus (S.) T. 1811.

Mesocottus Gracianow 1907.

Metacottus TALIEV 1946.

Microcottus SCHMIDT 1941.

Montereya HuвBs 1926.

Myoxocephalus (STELLER) Tilesius 1811.

Neohemilepidotus SaKaмото 1932. Ocynectes JORDAN et SNYDER 1904.

Oligocottus GIRARD 1856.

Onchocottus : emend. pro Oncocottus G. 1861.

Oncocottus GILL 1861.

Oxycottus J O R D A N et EvermanN 1898.

Paracottus.

Pegedictis RAFINESQUe 1820 : syn. de Cottus L. 1758.

Phallocottus SchULz 1938.

Phasmatocottus BoLIN 1936.

Phobetor KRÖYER 1845: syn. de Gymnacanthus S. 1839.

Podabrus RICHARDSON 1848 : pré-employé, syn. de Vellitor J. et S. 1894.

Potamocottus GILL 1861: syn. de Cottopsis G. 1851.

Prococottus GILL 1859.

Pseudoblennius Temminck et SCHLEGEL 1850.

Pseudoclinus Temminck et Schlegel 1850 : non valide.

Ptyonotus Günther 1860: syn. de Triglopsis G. 1854.

Radulinellus Bolin 1950: s./g. de Radulinus G. 1890: Icelidae.

Radulinopsis SOLDATOV et LINDBERG 1930.

Rheopresbe JoRdan et STARKs 1904.

Rusciculus GREeley 1901.

Rusulus Starks et MANN 1911. 
Scorpichthes BONAPARTE 1845: non valide, syn. de Myoxocephalus (S.) T. 1811.

Sigmistes RUTTER 1898.

Tauridea JORDAN et RICE 1878.

Taurocottus Soldatov et PAVLENKo 1915: syn. de Trichocottus S. et P. 1915.

Taurulus Gracianow 1907.

Thecopterus SMIтH 1904.

Trachidermis JORDAN 1919: vide Trachydermis H. 1837.

Trachydermis HECKEL 1837 : pré-employé, syn. de Centridermich thys R. 1844.

Trichocottus Soldatov et PAVLenKo 1915.

Triglopsis GIRARD 1854.

Uranidea DEKAY 1842.

Uranoscopus GRONOW 1763: non valide.

Vellitor Jordan et Starks 1904.

Zesticelus J O R D A N et EVERMANN 1896.

FAM. DES ABYSSOCOTTIDAE

Abyssocottus BERG 1906.

Asprocottus Berg 1906.

Batrachocottus BERG 1903.

Cottinella BERg 1907.

Limnocottus BERG 1906.

Linnocottus BERG 1906: errat. pro Limnocottus B. 1906.

Procottus Gracianow 1902.

FAM. DES ASCELICHTHYIDAE Ascelichthys JoRDAN et GILBERT 1880.

FAM. DES PSYCHROLUTIDAE

Eurymen GILBERT et BURKe 1912. Gilbertidia BERG 1899.

Gilbertina JORDAN et STARKS 1895 : pré-employé, syn. de Gilbertidia B. 1899.
Japanopsychrolutes NoJiмA 1937. Platycottus.

Psychrolutes GüNTHER 1861.

\section{FAMILLE DES}

NEOPHRYNICHTHYIDAE

Besnardia LAHILLE 1913.

Neophrynichthys GüNTHER 1876.

FAMILLE DES SYNCHIRIDAE Synchirus BEAN 1889.

FAMILLE DES EREUNIIDAE Ereunias JORDAN et SNYDER 1901.

FAM. DES RHAMPHOCOTTIDAE

Rhamphocottus GüNTHER 1874.

FAM. DES HEMITRIPTERIDAE

Hemitripterus CUVIER 1829.

Ulca JoRdan et EVERMANN 1896.

FAMILLE DES COTTOCOMEPHORIDAE

Baicalocottus BERG 1903: syn. de Cottocomephorus P. 1900.

Cottocomephorus Pellegrin 1900.

\section{FAMILLE DES COMEPHORIDAE}

Comephorus DE LACÉPÈDE 1800.

Elaeorhous Pallas 1811: syn. de Comephorus L. 1800.

\section{FAMILLE DES AGONIDAE}

(Souris-de-mer)

Acanthostelgis FOWLER 1959

Agonomalus GUICHENOT 1866.

Agonopsis GILL 1861.

Agonus Bloch et SchNeIder 1801. 
laspidophorus DE LACÉPÈDE 1802 : syn. d'Agonus B. et S. 1801.

Asterotheca Gilbert 1915.

Averruncus JoRdan et StaRKs 1895.

Bathyagonus GILBERT 1890.

Bothragonus (GILL) JORDAN et GILBERT 1882 .

Brachyopsis GILL 1861.

Cataphractus KLEIN 1777 : non valide.

Cataphractus (K.) Fleming 1828: syn. d'Agonus B. et S. 1801.

Cheiragonus HeRzENSTEIN 1890 : syn. d'Hypsagonus GILL 1861.

Draciscus JORDAN et SNYDER 1901.

Ganoideus WhITLEY 1950.

Hippocephalus SwaINSON 1839: syn. de Percis S. 1777.

Hypsagonus GILL 1861 .

Iburiella JoRDAN et HuBBS 1925.

Iburina JORDAN et HUBBS 1925 : s./g. de Occa J. et E. 1898.

Leptagonus GILL 1861.

Occa Jordan et Evermann 1898.

Occella JoRdan et HuBBs 1925.

Odontopyxis LOCKINGTON 1879.

Pallasia NARDo 1840.

Pallasina CRAMER 1895.

Paragonus Gill 1862 : syn. de Podothecus G. 1861.

Paragonus Guichenot 1869 : syn. de Podothecus G. 1861.

Paragonus RIBEIRo: pré-employé, syn. de Ribeiroa J. 1920.

Percis Scopoli 1777.

Phalangistes PALLAS 1811: syn. d'Agonus B. et S. 1801.

Podothecus GILL 1861.

Ribeiroa JORDAN 1920.

Sarritor CRAMER 1896.

Siphagonus STEINDACHNER 1876 : syn. de Brachyopsis G. 1861.

Stelgis Cramer 1896: syn. d'Acanthostelgis F. 1959.

Stelengicottus BOLIN 1936.

Stelengis JoRDAN et STARKS 1896 : pré-employé, syn. de Ganoideus W. 1958.
Stellerina CRAMER 1896.

Tilesina SCHMIDT 1904.

Xeneretmus (GILBERT) JORDAN 1903.

Xenochirus GILBERT 1890: pré-employé, syn. de Xeneretmus (G.) J. 1903.

Xystes Jordan et STARKs 1895.

\section{FAMILLE DES ASPIDOPHOROIDIDAE}

Anoplagonus GILL 1861.

Aspidophoroides DE LACÉPÈDE 1802.

Canthirhynchus SwaINSON 1839: syn. d'Aspidophoroides L. 1802.

Percis (SCOPOLI) RAFINESQUe 1815: syn. d'Aspidophoroides L. 1802.

Ulcina CRAMER 1896.

\section{FAM. DES CYCLOPTERIDAE} (Mollet, Gros seigneur, Lièvre-de-mer) Cyclolumpenus TANAKA 1912. Cyclopsis Popov 1931.

Cyclopterocottus POPOV 1930. Cyclopteroides Garman 1892.

Cyclopteropsis Soldatov et Popov 1929.

Cyclopterus LINNÉ 1758.

Eumicrotremus GILL 1862.

Lethotremus GILBERT 1895.

Lumpus Cuvier 1817: syn. de $\boldsymbol{C y}$ clopterus L. 1758.

Lumpus RAFINESQUE 1815: non valide.

Lumpus OKen 1782: syn. de $\boldsymbol{C y}$ clopterus L. 1758.

Oncotion KLEIN 1777 : non valide.

Pelagocyclus LINDBERG et LegezA 1955.

FAMILLE DES LIPAROPIDAE

Aptocyclus de La PylaIE 1835.

Cyclopterichthys STEINDACHNER 1881: syn. d'Atopocyclus P. 1835 . 
Liparoides LLLOYD 1909.

Liparops GARMAN 1892.

\section{FAMILLE DES LIPARIDAE}

(= Cyclogasteridae $)$

(Marmotte-de-mer, Limace-de-mer)

Acantholiparis GILBERT et BURKE 1912.

Allinectes J O R D A N et EvermanN 1898

Allochir JoRdan et EVERMANN 1896.

Allurus JORDAN et EVERMANN 1896: pré-employé, syn. d'Allinectes J. et E. 1898 .

Amitra Goode 1880: pré-employé, syn. de Paraliparis C. 1878.

Amitrichthys JORDAN et EVERMANN 1896: syn. de Paraliparis C. 1878 .

Bathyphasma GILBERT 1895.

Careliparis Garman 1892.

Caremitra J o R D A N et EvermanN 1896.

Careproctus KRÖYER 1861.

Crystallias JORDAN et SNYDER 1902.

Crystallichthys JORDAN et GILBERT 1898.

Cyclogaster Gronow 1763 : non valide.

Cyclogaster (G.) BURKE 1912: syn. de Liparis S. 1777.

Elassodiscus GILBERT et BURKE 1912

Elephantichthys HuBBS et SchULTZ 1934.

Enantioliparis VAILlant 1888.

Gymnolycodes VAILlant 1888.

Gyrinichthys GILBERT 1895.

Hilgendorfia GoODE et BEAN 1895.

Liparis SCOPOLI 1777.

Liparis RösE 1793 : syn. de Liparis S. 1777.

Lipariscus GILBERT 1915.

Liparius RAFINESQUE 1815 : non valide.

Lyoliparis JORDAN et EVERMANN 1896.
Massaria Gistel 1848 : syn. de Liparis S. 1777.

Monomitra Goode 1883: syn. de Paraliparis C. 1878.

Nectoliparis GILBERT et BURKE 1912: syn. de Paraliparis C. 1878.

Neoliparis STEINDACHNER 1875.

Paraliparis COLlett 1878.

Polypera BURKe 1912.

Prognutus Jordan et Gilbert 1898.

Psednos BARNARD 1927.

Pseudoliparis ANDRIYASHEW 1955 : s./g. de Careproctus K. 1861.

Rhinoliparis GILBERT 1895.

Temnocara BURKE 1930.

Trimegistus JORDAN et SNYDER 1904

FAM. DES EUTELICHTHYIDAE

Eutelichthys TORTONESE 1959.

FAMILLE DES

MARUKAWICHTHYIDAE

Marukawichthys SАKАMOTO 1931.

\section{Sous-ordre des \\ DACTYLOPTEROIDEI}

FAM. DES DACTYLOPTERIDAE (= Cephalacanthidae)

(Grondins volants)

Cephacandia RAFINESQUe 1815 : syn. de Dactylopterus L. 1803.

Cephalacanthus DE LACÉPÈDE 1803 : juvénile de Dactylopterus L. 1803.

Dactyloptena JORDAN et RICHARDSON 1908.

Dactylopterus DE LACÉPÈDE 1803.

Daicocus JORDAN et RICHARDSON 1908.

Ebisinus JORDAN et RICHARDSON 1908.

Gonocephalus Gronow 1854: syn. de Dactylopterus L. 1803. 


\section{Sous-ordre des PEGASOIDEI}

\section{FAMILLE DES PEGASIDAE}

(Pégases-dragons)

Acanthopegasus Mc CULLOCH 1915. Cataphractus GRONOW 1763: non valide.

Eurypegasus BLEEKER 1863 : syn. de Pegasus L. 1758.

Leptopegasus BLEEKER 1873 : syn. de Parapegasus B. 1870.

Parapegasus BLEEKER 1870.

Pegasus LinNÉ 1758.

Zalistes JoRDAN et SNYDER 1901 : syn. de Pegasus L. 1758.

\section{Ordre des \\ PLEURONECTIFORMES}

Sous-ordre des PSETTODOIDEI

\section{FAMILLE DES PSETTODIDAE}

Psettodes BenNetT 1830.

Sphagomorus COPE 1867.

\section{Sous-ordre des PLEURONECTOIDEI}

FAMILLE DES BOTHIDAE

(Turbots et Barbues)

Achiropsetta Norman 1930. Anticitharus GÜNTHER 1880.

Apsetta KYLE 1900.

Aramaca JORDAN et Goss 1885 : syn. de Syacium R. 1840.

Arnoglossus BLEeKer 1862.

Asterorhombus TANAKa 1915.

Azevia JoRdAN et Goss 1888.

Bascanius SchIöDTE 1868: syn. de Chascanopsetta A. 1894.

Boopsetta ALCock 1896.
Bothus RAfinesque 1810.

Brachypleurops FOWLER 1934.

Caulopsetta Gill 1893 : syn. d'Arnoglossus $\mathrm{B} .1862$.

Charybdia FACCIOLA 1885: larve de Platophrys S. 1839.

Chascanopsetta ALCock 1894.

Chopinopsetta WhITLEY 1931: syn. de Citharus R. 1793.

Citharichthys BLEEKER 1862.

Citharoides HuBBs 1915.

Citharus Röse 1793.

Citharus BLEEKER 1862: pré-employé, syn. de Citharus R. 1793.

Coccolus (Bonaparte) Cocco 1844 : larve de Platophrys S. 1839.

Crossobothus FowLER 1934: s./g. de Bothus R. 1810.

Crossolepis Norman 1927: syn. de Psettina H. 1915.

Crossorhombus Regan 1920.

Cyclopsetta GILL 1888.

Delothyris Goode 1883: larve de Monolene G. 1880.

Dollfusetta WHITLEY 1950: s./g. d'Arnoglossus B. 1862.

Dollfusina Chabanaud 1933: préemployé, syn. de Dolfusetta W. 1950.

Embassichthys JoRDAN et EVERMANN 1896.

Engyophrys JORDAN et BOLLMAN 1889.

Engyprosopon GÜNTHER 1862.

Etropus JoRDAN et GILBERT 1881: syn. de Citharichthys B. 1862.

Eucitharus GILl 1888: syn. de Citharus R. 1793.

Europus KLEIN 1775 : non valide.

Grammatobothus NORMAN 1926.

Hemirhombus BLEEKER 1862: syn. de Syacium R. 1840.

Imhoffichthys WHITLEY 1951.

Istiorhombus WHITLEY 1931.

Kamoharaia KURONUMA 1940.

Kyleia Chabanaud 1933.

Laeops GüNTHER 1880.

Laeoptichthys HUBBS 1915. 
Laiopteryx WEBER 1913.

Lambdopsetta SMITH et POPE 1906. Lepidoblepharon WEBER 1913.

Lepidopsetta GüNTHER 1880: préemployé, syn. de Mancopsetta G. 1905 .

Lepidorhombus GÜNTHER 1862.

Leptolaeops FOWLER 1934 : s./g. de Laeops G. 1880.

Liopteryx : errat. pro Laiopteryx W. 1913.

Lophonectes GÜNTHER 1880.

Lophopsetta GILL 1861.

Lophorhombus MACLEAY 1882: syn. de Lophonectes G. 1880.

Mancopsetta GILL 1905.

Metoponops GILL 1864: syn. d'Orthopsetta G. 1862.

Monolene Goode 1880.

Vematops GüNTHER 1880.

Orthopsetta GILL 1862.

Parabothus NORMAN 1931.

Paracitharus REgAN 1920.

Passer (KLeIN) VAlenciennes 1855 : pré-employé, syn. de Platophrys S. 1839 .

Pelecanichthys Gilbert et CRAMER 1897.

Peloria Cocco 1844 : larve de Platophrys S. 1839.

Perissias Jordan et Evermann 1898.

Phrynorhombus GüNTHER 1862.

Plagiopsetta Franz 1910.

Platophrys SWAINSON 1839.

Platotichthys Nichols 1921.

Psetta KLEIN 1775 : non valide.

Psetta Swainson 1839.

Psettina HubBs 1915.

Psettylis Alcock 1890.

Pseudocitharichthys WEBER 1913. Rhomboides GoldFuss 1820: syn. de Bothus R. 1810.

Rhomboidichthys BLEEKER 1856: syn. de Platophrys S. 1839.

Rhombus Cuvier 1817 : syn. de Bothus R. 1810.

Rhombus KLEIN 1775: non valide, syn. de Bothus R. 1810.
Scaeops Jordan et Starks 1904.

Scianectes ALCOCK 1890.

Scidorhombus TANAKA 1915.

Scophthalmus RAFINESQUE 1810.

Solea CATESBY 1771: non valide.

Syacium RANZANI 1840.

Symboulichthys CHABANAUD 1927.

Taeniopsetta GILBERT 1905.

Thyris Goode 1880: pré-employé, larve de Monolene G. 1880.

Thysanopsetta GÜNTHER 1880.

Trachypterophrys FRANZ 1910: syn. de Chascanopsetta A. 1894.

Trichopsetta GILL 1888.

Zeugopetrus GoTTSCHE 1835.

\section{FAM. DES PARALICHTHYIDAE}

Ancylopsetta Gill 1863: syn. de Pseudorhombus B. 1862.

Chaenopsetta GILL 1861: syn. de Paralichthys G. 1859.

Gastropsetta GoOde et BEAN 1894. Hippoglossina STEINDACHNER 1876. Lioglossina GILBERT 1890.

Neorhombus de CASTELnaU 1875.

Notosema GoODE et BEAN 1882.

Paralichthys GIRARD 1859.

Pseudorhombus BLEEKER 1862.

Ramularia JORDAN et EVERMANN 1898.

Rhombiscus JORDAN et SNYDER 1900 : s./g. de Pseudorhombus B. 1862 .

Spinirhombus Oshima 1928: syn. de Pseudorhombus B. 1862.

Tarphops JORDAN et THOMPSON 1914.

Tephrinectes GüNTHER 1862.

Tephritis GüNTHER 1862: pré-employé, syn. de Tephrinectes G. 1862.

Teratorhombus MACLEAY 1881 : syn. de Pseudorhombus B. 1862.

Uropsetta GILL 1862: syn. de Paralichthys G. 1859.

Velifracta Jordan 1907 : syn. de Te. phrinectes G. 1862. 
Verecundum JORDAN 1890.

Xystreurys JoRdAN et GILBERT 1890.

\section{FAM. DES HIPPOGLOSSIDAE}

(Flétan ou Halibut) •

Acanthopsetta ScHMIDT 1904.

Araias Jordan et Starks 1904 : syn. de Dexistes Jordan et Starks 1904.

Atheresthes JORDAN et GILBERT 1880.

Citharus ReINHARDT 1838: syn. d'Hippoglossoides G. 1835.

Cleisthenes JORDAN et STARKs 1904. Cynopsetta Schmidt 1903.

Dexistes JoRdan et StaRKS 1904.

Drepanopsetta GILL 1861: syn. d'Hippoglossoides G. 1835.

Eopsetta JoRDAN et Goss 1885.

Hippoglossoides GotTSCHE 1835.

Hippoglossus CUVIER 1817.

Lyopsetta JoRDAN et Goss 1885 .

Platysomatichthys BLEEKER 1862 : syn. de Reinhardtius GILL 1861.

Pomatopsetta GrL.L 1864 : syn. d'Hippoglossoides G. 1835 .

Protopsetta SchMIDT 1904.

Reinhardtius GILL 1861.

Veraequa Jordan et Starks 1904.

Verasper Jordan et GILBERT 1898.

Xystrias JORDAN et STARKS 1904: syn. d'Eopsetta J. et G. 1885.

\section{FAM. DES PLEURONECTIDAE}

(Flet, Plie, Carrelet, Limande)

Alaeops Jordan et Starks 1904.

Brachyprosopon BLEEKER 1862: syn. de Cynocoglossus B. 1837.

Clidoderma BLEEKER 1862.

Curioptera WHITLEY 1951.

Cynocoglossus Bonaparte 1837.

Cynoglossa Bonaparte 1845 : syn. de Cynicoglossus B. 1837.

Errex JORDAN 1919.
Euchalarodus GILL 1864: syn. de Liopsetta G. 1864.

Flesus Moreau 1881 : syn. de Platychthys G. 1854.

Gareus HubBs 1915.

Glyptocephalus GoTTSCHE 1835.

Heteroprosopon BLEEKER 1862: syn. de Pleuronichthys G. 1844.

Hypsopsetta GILL 1862.

Inopsetta JORDAN et Goss 1885.

Isopsetta LOCKINGTON 1882.

Kareius JORDAN et SNYDER 1900 : syn. de Platychthys G. 1854.

Lepidopsetta GILL 1862.

Limanda GoTTSCHE 1835.

Limandella JoRDAN et STARKS 1906 : syn. de Pseudopleuronectes B. 1862.

Liopsetta GILL 1864.

Marleyella FOWLER 1925 : s./g. de Poecilopsetta G. 1880.

Microstomus GotTSCHE 1835 : syn. de Cynicoglossus B. 1837.

Myzopsetta GiLL 1861 : syn. de Limanda G. 1835.

Neoetropus Hildebrand et SchroeDER 1928.

Oncopterus STEINDACHNER 1875 : syn. de Curioptera W. 1951.

Paralimanda BREDER 1927 : syn. de Poecilopsetta G. 1880.

Parophrys GIRARD 1854.

Passer KLEIN 1775: non valide.

Platessa Cuvier 1817: syn. de Pleuronectes L. 1758 .

Platichthys GIRARD 1854.

Pleuronectes LINNÉ 1758.

Pleuronichthys GIRARD 1844.

Pluviopsetta TANAKA 1916.

Poecilopsetta GÜNTHER 1880.

Psettichthys GIRARD 1854.

Pseudoplatichthys HIKITA 1934.

Pseudopleuronectes BLEEKER 1862.

Tanakius HubBs 1918: s./g. de $\boldsymbol{C y}$ nicoglossus B. 1837. 
FAMILLE DES SAMARIDAE (= Paralichthodidae)

Brachypleura GüNTHER 1862. Paralichthodes GILCHRIST 1902. Samaris GRAY 1831.

Samariscus GII.BERT 1905.

\section{FAM. DES RHOMBOSOLEIDAE}

Adamasoma Whitley et Phillipps 1939: s./g. de Rhombosolea G. 1862 .

Ammotretis GüNTHER 1862.

Azygopus Norman 1926.

Colistium NORMAN 1926.

Peltorhamphus GüNTHER 1862.

Psammodiscus GüNTHER 1862.

Rhombosolea GÜNTHER 1862.

\section{Sous-ordre des SOLEOIDEI}

\section{FAMILLE DES ACHIRIDAE}

Achiropsis SteIndachner 1876.

Achirus de Lacépède 1803.

Achirus Chabanaud 1928: syn. de Trinectes R. 1832 .

Amate Jordan et Starks 1906.

Anathyridium ChABANAUD 1928: s./g. de Baiostoma B. 1882.

Apionichthys KAUP 1858.

Baeostoma : vide Baiostoma B. 1882.

Baiostoma BEAN 1882.

Catathyridium CHABANAUD 1928: s./g. de Baiostoma B. 1882.

Grammichthys KAUP 1858: syn. d'Achirus L. 1803.

Gymnachirus KAUP 1858.

Heteromycteris : syn. d'Amate J. et S. 1906.

Hypoclinemus Chabanaud 1928.

Monochirus Kaup 1858 : syn. d'Achirus L. 1803.

Nodogymnus Chabanaud 1928.

Normanetta WHITLEY 1931.
Pnictes Jordan 1919.

Rendahlia Chabanaud 1930.

Soleonasus EIGENMANN 1912.

Soleotalpa G ü N T H E R 1862: syn. d'Apionichthys K. 1858.

Trinectes RAFINESQUE 1832.

Typhlachirus HARDENBERG 1932.

\section{FAMILLE DES SOLEIDAE (Soles)}

Aseraggodes Kaup 1858.

Austroglossus REgAN 1920.

Barbourichthys CHABANAUD 1934.

Barnardichthys CHABANAUD 1927.

Bathysolea Roule 1916.

Beaufortella CHABANAUD 1943.

Bowenia HAAST 1873.

Buglossidium CHABANAUD 1930.

Buglossus GüNTHER 1862: syn. de Microchirus R. 1814.

Capartella ChaBANAUd 1950.

Coryphillus ChaBanaud 1931.

Dicologlossa Chabanaud 1927.

Dicologoglossa : vide Dicologlossa C. 1927.

Echinosolea ChaBANAUd 1927.

Eusolea Roule 1919: validité douteuse.

Haplozebrias ChaBANaUd 1943.

Heteromycteris KAUP 1858.

Leptosoma NARDO 1827: pré-employé, syn. de Monochirus R. 1814.

Liachirus GÜNTHER 1862.

Microbuglossus GüNTHER 1862.

Microchiropsis Chabanaud 1956.

Microchirus RAFINESQUE 1814.

Microchirus BONAPARTE 1840: syn. de Microchirus R. 1814.

Monochir Cuvier 1829: syn. de Microchirus R. 1814.

Monochirus (CUVIER) OKEN 1817.

Monochirus BONAPARTE 1837: syn. de Microchirus R. 1814.

Monodichthys ChaBanaud 1926 : syn. d'Heteromycteris K. 1858. 
Nematozebrias ChaBANAUd 1943.

Odontolepis FISCHER 1813: syn. de Monochirus (C.) O. 1817 ou de Symphurus R. 1810.

Pardachirus GüNTher 1862.

Pegusa GüNTHER 1862.

Pelotretis WAITE 1911.

Phyllichthys Mc Culloch 1916.

Pseudaustroglossus CHABANAUD 1937.

Quenselia JORDAN 1888.

Rhinosolea FOWLER 1946.

Solea KLEIN 1775 : non valide.

Solea Quensel 1806.

Solea RAFInESQue 1810 : syn. de Solea Q. 1806.

Solea Cuvier 1817: syn. de Solea Q. 1806.

Spanius Gistel 1848 : syn. de Monochirus (C.) O. 1817.

Strabozebrias Chabanaud 1943.

Synclidopus ChaBanaud 1943.

Trichobrachirus CHABANAUD 1943.

Vanstraelenia CHABANAUD 1950.

Xenobuglossus ChaBanaud 1950.

Zevaia Chabanaud 1943.

\section{FAMILLE DES SYNAPTURIDAE}

Achiroides BLEeKER 1851.

Achylopa Whitley 1947 : syn. d'Euryglossa K. 1858.

Aesopia KaUp 1858.

Anisochirus GüNTHER 1862.

Austroglossus Regan 1920.

Brachirus SwaInson 1839 : syn. d'Euryglossa K. 1858.

Brachyrus SwaINSON 1839: syn. de Synaptura C. 1849.

Chabanaudetta WHITLEY 1931.

Coryphaesopia CHABANAUd 1930.

Dendrochirus SWaINSON 1839: syn. d'Euryglossa K. 1858.

Dexillichthys WhItLey 1931.

Dexillus Chabanaud 1930: pré-employé, syn. de Dexillichthys W. 1931.

Euryglossa KAUP 1858.
Eurypleura KAUP 1858: syn. d'Euryglossa K. 1858.

Heterobuglossus ChaBanaud 1931. Holonodus ChaBAnaUd 1938: s./g. de Zebrias J. et S. 1900.

Mischominatus CHABANAUD 1938: s./g. d'Euryglossa K. 1858.

Mischommata : syn. de Dexillichthys W. 1931.

Mischommatus : vide Mischommata.

Paradicula WhITLEY 1931.

Pseudoaesopia Chabanaud 1934 : s./g. de Zebrias J. et S. 1900.

Soleichthys BLEEKER 1860: syn. d'Aesopia K. 1858.

Strandichthys WhITLEY 1937: syn. de Dexillichthys W. 1931.

Synaptura CANTOR 1849.

Synapturichthys CHABANAUD 1927. Whitleyia Chabanaud 1930 : pré-employé, syn. de Paradicula W. 1931 .

Zebrias JORDAN et SNYDER 1900.

\section{FAM. DES CYNOGLOSSIDAE}

Acedia Jordan 1888.

Ammopleurops GüNTHER 1862: syn. de Symphurus R. 1810.

Aphoristia Kaup 1858 : syn. de Symphurus R. 1810.

Arelia KAUP 1858.

Areliscus JoRdAN et SNYDER 1900.

Bibronia Cocco 1844 : larve de Sym. phurus R. 1810.

Cantoria KauP 1858: pré-employé, syn. de Cantorusia W. 1940.

Cantorusia WhItLey 1940.

Compsomidiama CHABANAUD 1951.

Cynoglossoides vON BONDE 1922.

Cynoglossoides SMITH 1949: pré-employé, syn. de Notrullus W. 1951.

Cynoglossus Hamilton - Buchanan 1822.

Dexiourius ChaBanaUd 1947.

Dollfusichthys CHABANAUd 1931.

Eupnoea GISTEL 1848 : syn. de Symphurus R. 1810. 
Euprorista Gistel 1848: syn. de Symphurus R. 1810.

Glossichthys GILL 1861: syn. de Symphurus R. 1810.

Grammichthys KAUP 1858.

Icania KAUP 1858.

Notrullus WhITLEy 1951.

Paraplagusia BleEKER 1865.

Plagiusia JoRdan 1919 : vide Plagusia B. 1845 .

Plagusia Browne 1789 : non valide.

Plagusia RAFINESQUE 1815: non valide.

Plagusia (B.) Cuvier 1817 : syn. de Symphurus R. 1810.

Plagusia Swainson 1839: syn. de Paraplagusia B. 1865.

Plagusia Bonaparte 1845: syn. de Symphurus R. 1810.

Rhinoplagusia BLEEKER 1870. Symphurus RAFINESQUE 1810.

Trulla KaUP 1858.

Usinosita JORDAN et SNYDER 1900 : syn. de Rhinoplagusia B. 1870. Usinostia : errat. pro Unsinosita J. et S. 1900 .

\section{Ordre des MASTACEMBELIFORMES}

Sous-ordre des
CHAUDHURIOIDEI

FAM. DES CHAUDHURIIDAE Chaudhuria ANNANDALE 1918.

\section{Sous-ordre des MAST ACEMBELOIDEI}

FAM. DES MASTACEMBELIDAE

Bdellorhynchus JORDAN et TANAKA 1927.

Caecomastacembelus POLL 1958.
Macrognathus DE LACÉPÈDE 1800 . Mastacembelus GRONOW 1763: non valide.

Mastacembelus SCOPOLI 1777.

Pararhynchobdella BLEEKER 1874. Rhynchobdella BLOCH et SCHNEIDER 1801: syn. de Macrognathus L. 1800 .

\section{Ordre des LOPHIIFORMES}

\section{Sous-ordre des LOPHIOIDEI}

\section{FAMILLE DES LOPHIIDAE}

(Baudroies, Lotes-de-mer)

Batrachus KLEIN 1775: non valide, syn. de Lophius L. 1758.

Chirolophius REGAN 1903: syn. de Lophiodes G. et B. 1895.

Chironectes RAFINESQUE 1815: syn. de Lophius L. 1758.

Conomus RAFINESQUE 1815 : syn. de Lophius L. 1758.

Discolophius FOWLER 1944.

Lophidius RAFINESQUE 1815 : syn. de Lophius L. 1758.

Lophiodes GoOdE et BEAN 1895.

Lophiomus GILL 1882.

Lophiopsides GUICHENOT 1867.

Lophius LINNÉ 1758.

Sladenia REGAN 1908.

\section{Sous-ordre des ANTENNARIOIDEI}

FAMILLE DES ANTENNARIIDAE

Abantennarius SchULTZ 1957.

Antennarius (COMMERSON) DE LACÉPÈDE 1798: non valide.

Antennarius (C.) CUVIER 1817.

Antennatus Schultz 1957.

Batrachops JoRDAN 1917 : errat. pro Batrachopus G. 1820. 
Batrachopus Goldfuss 1820: syn. d'Antennarius (C.) C. 1817.

Capellaria GISTEL 1848: syn. d'Antennarius (C.) C. 1817.

Chironectes CUVIER 1817: pré-employé, syn. d'Antennarius (C.) C. 1817.

Echinophryne Mc CULloch 1918.

Fowlerichthys BARBOUR 1941 : s./g. d'Antennarius (C.) C. 1817.

Golem Whitley 1957.

Histrio FISCHER 1813.

Histriophryne GILL 1863.

Kanazawaichthys Schultz 1957.

Lophiocharodon : errat. pro LophioCharon W. 1933.

Lophiocharon WHITLEY 1933.

Nudiañtennarius SchULtz 1957.

Phrynelox WhitLey 1931.

Plumantennatus SchULTZ 1957: s./g. d'Antennarius (C.) C. 1817.

Pterophryne GILl. 1863: pré-employé, syn. de Histrio F. 1813.

Pterophrynoides GILL 1878: syn. d'Histrio F. 1813.

Pterophrynus: vide Pterophryne G. 1863.

Saccarius GÜNTHER 1861.

Tetrabrachium GüNTHER 1880.

Trianectes Mc Culloch 1918.

Triantennatus SCHULTZ 1957 : s /g. de Phrynelox W. 1931.

Trichophryne Mc CuLloch 1918.

Uniantennatus SCHULTZ 1957 : s./g. de Lophiocharon W. 1933.

Xenophrynichthys ScHULTZ 1957: s./g. d'Antennatus S. 1957.

FAMILLE DES

BRACHIONICHTHYIDAE

Brachionichthys BLEEKER 1855.

Brachyonichthys: vide Brachionichthys B. 1855.

Sympterichthys GILL 1878.
FAMILLE DES CHAUNACIDAE

Chaunax LOWE 1846.

Sous-ordre des OGCOCEPHALOIDEI

FAM. DES OGCOCEPHALIDAE

$$
\text { (= Malthidae) }
$$

(Chauve-souris-de-mer)

Astrocanthus Swainson 1839: syn. d'Halieutaea C. et V. 1837.

Brephostoma ALCOCK 1889.

Coelophrys BRAUER 1902.

Dibranchichthys GARMAN 1899.

Dibranchopsis GARMAN 1899.

Dibranchus Peters 1875.

Halicmetus ALCOCK 1891.

Halieutaea CUVIER et VALENCIENNES 1837.

Halieutella GoOdE et BEAN 1885 .

Halieutichthys POEY 1863.

Halieutopsis GARMAN 1899.

Malthae: emend. pro Malthe C. 1817.

Malthe Cuvier 1817 : syn. d'Ogcocephalus F. 1813.

Malthopsis AlcocK 1891.

Ogcocephalus FISCHER 1813.

Oncocephalus : corrig. pro Ogcocephalus F. 1813.

Rhycherus OGILBY 1907.

Tathicarpus OGILBY 1907.

Zalieutes JoRDAN et EVERMANN 1896.

\section{Sous-ordre des CERATIOIDEI}

FAM. DES MELANOCETIDAE

Lyocetus GÜNTHER 1887.

Melanocetus GüNTHER 1864.

Xenoceratias Regan et Trewavas 1932. 
FAM. DES HIMANTOLOPHIDAE

Aegonichthys Clarke 1878.

Corynolophus GILL 1878.

Corynophorus Osorio 1912.

Himantolophus REINHARDT 1838.

Lipactis REgAN 1925.

Paroneirodes Alcock 1890.

Pentherichthys Regan et Trewavas 1932.

Tyrannophryne REGAN et TREWAVAS 1932.

\section{FAMILLE DES ONEIRODIDAE}

Bertelsenna WHITLEY 1954.

Chaenophryne REGAN 1925.

Danaphryne BERTELSEN 1951.

Lasiognathus REgAN 1925.

Lophodolos LLOOYD 1909.

Oneirodes LÜTKEN 1871.

Oneiroides : errat. pro Oneirodes L. 1871.

Spiniphryne BERTELSEN 1951: syn. de Bertelsenna W. 1954.

Trematorhynchus REgAN et TREWAVAS 1932.

FAM. DES GIGANTACTINIDAE

Gigantactis BRAUER 1902.

FAM. DES CAULOPHRYNIDAE

Caulophryne GoODE et BEAN 1895.

\section{FAMILLE DES CERATIIDAE}

Acentrophryne REGAN 1926.

Amacrodon Regan et Trewayas 1932.

Anomalophryne REgAN et TREWAVAS 1932.

Bathyceratias BEEBE 1934.

Borophryne REGAN 1925.

Caranactis REgan et Trewavas 1932.
Centrocetus Regan et Trewavas 1932.

Ceratias KRÖYER 1845.

Ceratocaulophryne ROULE et ANGEL 1932.

Chirophryne Regan et Trewavas 1932.

Cryptopsaras GILL 1883.

Ctenochirichthys REgAN et TREWAVAS 1932.

Dermatias RADCLIFFE 1912: s./g. de Dolopichthys G. 1899.

Dolopichthys GARMAN 1899.

Edriolychnus REGAN 1925.

Laevoceratias PARR 1927.

Leptacanthichthys REgAN et TREWavas 1932 : s./g. de Dolopichthys G. 1899.

Mancalias GILl 1878 : syn. de Ceratias K. 1845.

Microlophichthys Regan et TrewaVAS 1932: s./g. de Dolopichthys G. 1899.

Miopsaras GILBERT 1905.

Monoceratias GILBERT 1915.

Nannoceratias REgan et Trewavas 1932.

Paraceratias TANAKA 1908.

Parrichthys BARBOUR 1942.

Pentherichthys Regan et Trewavas 1932.

Photocorynus REgAN 1925.

Reganichthys BIGELOW et BARBOUR 1944 : pré-employé, syn. de $\boldsymbol{R e}$ ganula B. et B. 1944.

Reganula BIGELOW et BARBOUR 1944.

Rhynchactis REGAN 1925.

Rhynchoceratias REGAN 1925.

Teleotrema REgAN et Trewavas 1932.

Thaumatichthys SMITH et RADCLIFFE 1912.

Typhlopsaras GILL 1883: syn. de Ceratias G. 1878.

Typhoceratias BARBOUR 1942. 
FAMILLE DES NEOCERATIIDAE

Neoceratias PAPPENHEIM 1914.

FAMILLE DES LINOPHRYNIDAE

Allector Heller et SNodgrass 1903.

Cryptolynchus Regan et Trewavas

1932: syn. d'Allector H. et S. 1903.

Diabolidium BEEBE 1926.

Haplophryne REGAN 1912.

Linophryne COLLetT 1886.
FAMILLE DES ACERATIIDAE Aceratias BRAUER 1902.

Hyaloceratias KOEFOED 1944.

FAMILLE DES

AESCHYNICHTHYIDAE nom. nov. (= Diceratiidae)

Aeschynichthys OGILBy 1907.

Bufoceratias WHITLEY 1931.

Diceratias GüNTHER 1887: pré-employé, syn. d'Aeschynichthys $\mathrm{O}$. 1907.

Phrynichthys Pietschman 1927 : préemployé, syn. de Bufoceratias W. 1931.

\section{Sous-classe des \\ Brachiopterygiens (Brachyopterigia)}

Ordre des POLYPTERIFORMES

FAMILLE DES POLYPTERIDAE (Bichir)

Polypterus Geoffroy - St - Hillaire 1798.
FAMILLE DES

CALAMOICHTHYIDAE

(Poisson-roseau)

Calamoichthys SмIтн 1866.

Erpetoichthys SMITH 1865: syn. de Calamoichthys S. 1866.

Herpetoichthys (S.) GüNTHER 1865 : pré-employé, syn. de Calamoichthys S. 1866.

\section{Sous-classe des Dipneustes (Dipneustia)}

Ordre des CERATODIFORMES

FAMILLE DES CERATODIDAE (= Ceratodontidae) (Barramunda)

(Le genre type de la famille, Ceratodus est fossile).

Epiceratodus Teller 1891: syn. de Neoceratodus C. 1876.

Ompax de Castelnau 1879: validité douteuse.

Neoceratodus DE CASTelnau 1876.
FAM. DES LEPIDOSIRENIDAE (Lolach)

Amphibichthys Hogg 1841: syn. de Lepidosiren F. 1837.

Lepidosiren FITZINGER 1837.

FAMILLE DES PROTOPTERIDAE Protomelus Hogg 1841 : syn. de Protopterus O. 1839.

Protopterus OWEN 1839.

Rhinocryptis PeTERS 1844: pré-employé, syn. de Protopterus O. 1839. 


\section{Sous-classe des}

\section{Crossopterygiens (Crossopterygia)}

\section{Ordre des COELACANTHIFORMES}

FAM. DES COELACANTHIDAE

Latimeria SMITH 1941.

Malania SмrтH 1953 : syn. de Latimeria S. 1941. 


\section{TABLE ALPHABÉTIQUE}

(les chiffres renvoient aux pages du catalogue)

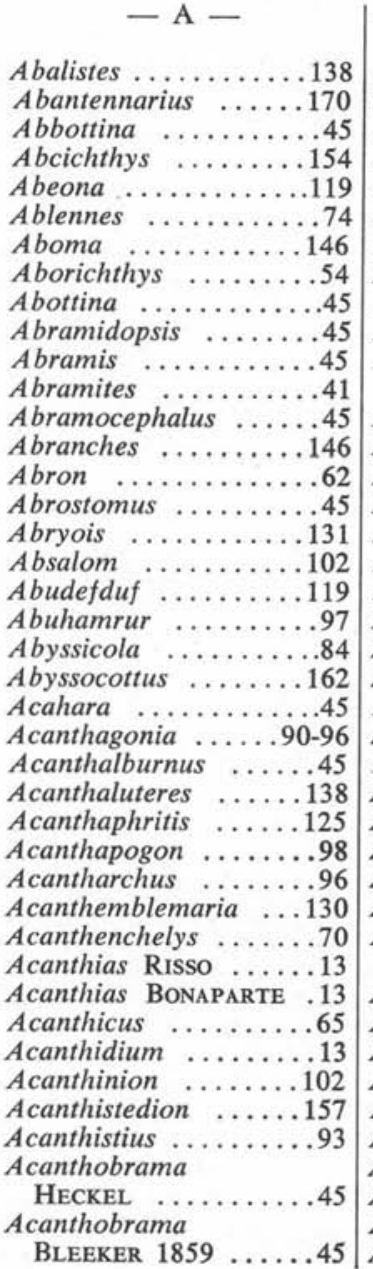

Acanthobrama
BLEEKER $1865 \quad \ldots .45$ Acanthocaulus ........136 Acanthocephalus ...79-93 Acanthocepola .......118 Acanthochaenus .....89 Acanthochaetodon ...114 Acanthocharax .......36 Acanthochromis ....119 Acanthocirrhitus .....118 Acanthocleithron ....61 Acanthoclinus JENYNS .95 Acanthoclinus

MocQuard ........129

Acanthocobitis .......54

Acanthocottus .......161

Acanthocybium ......139

Acanthocemus ..........

Acanthodemus .....65

Acanthoderma ......141

Acanthodermus ....6.65

Acanthodoras .......57

Acanthognathus ......82

Acanthogobio ......45

Acanthogobius .....146

Acantholabrus ......121

Acantholatris .......119

Acantholebius .......158

Acantholepis .......25

Acantholiparis .......164

Acantholumpenus ....131

Acanthomullus .....110 Acanthonotus (T.)

DAY ...........45 Acanthonotus Gray ..60 Acanthonotus BLOCH ..73 Acanthonus .........134 Acanthopagrus .......111 Acanthopegasus ....165 Acanthoperca ......97 Acanthophthalmus ....54 Acanthoplesiops .....95 Acanthoplichthys ...159 Acanthopodus .......112
Acanthopolyipnus ...27

Acanthopoma ......64

Acanthopsetta ........167

Acanthopsis

Van Hasselt .....54

Acanthopsis Agassiz ..54

Acanthopsoides .....54

Acanthorhinus ......13

Acanthorhodeus .....45

Acanthorutilus .......45

Acanthosoma .......36

Acanthosphex ......159

Acanthostelgis .....162

Acanthostethus ......76

Acanthostracion ......33

Acanthurus .........137

Acapoeta ..........45

Acara ............115

Acarana ............33

Acarauna CATESBY ..114 Acarauna

Sewastianof $\ldots \ldots 122$

Acarichthys .........115

Acaronia .........115

Acaropsis ............115

Acedia ...........169

Acentrachme .......73

Acentrogobius .......146

Acentrolophus ......142

Acentronichthys.....62

Acentronura .......82

Acentrophryne .....172

Aceratias .........173

Acerina GülLDENSTäDT .100

Acerina CUVIER 1817.100

Acerina CUVIER 1820.100

Acestra JARDINE .....82

Acestra KNER .......65

Acestridium .......65

Acestrocephalus .....36

Acestrorhamphus .....36

Acestrorhynchus ......36

Acharnes ..........115

Acheilognathus .......45 
Achiroides ..........169 Achirophichthys .....70 Achiropsetta .......165 Achiropsis .........168 Achirostomias .....28 Achirus ..........168 Achoerodus ........121 Achylopa ..........169 Acinacea ..........141 Acipenser . . . . . . . 18 Acmonotus ........82 Acnodon ............36 Acomus ...........44 Aconichthys .......126 Acoura ...........54 Acourus ...........54 Acra ............45 Acreichthys .......138 Acrobrycon .........36 Acrocheilichthys .....45 Acrocheilus .........45 Acrochordonichthys ...60 Acronurus .........137 Acroperca ........991 Acropoecilia .......80 Acropoma T. et S. ...97 Acropoma .........139 Acrossochilus ......45 Acroteriobatus .......14 Acrotus ..........143 Acteis .............129 Actenolepis ........93 Actinicola .........119 Actinoberyx .......88 Actinochromis ......119 Actinogobius ........146 Acus MüLler ......82 Acus Valmont .....8.82 Acus Catesby .......19 Acus Plumier .......76 Acus Swainson .....8.82 Acuticurimata .......41 Acutomentum .......154 Acutospina ........154 Acyrtops ...........153 Acyrtus ..........153 Adamacypris ......45 Adamasoma ........168 Adenapogon .......98 Adenops ...........77 Adinia ............79 Adiniops .........79 Adioryx ..........88 Adiposia .........56 Adonis ...........127 Adrianichthys .......82 Adventor ..........154 Aeglefinus ..........85 Aegonichthys .....172 Aelurichthys .......56
Aemasia .........66 Acoliscus .........73 Aequidens

STEINDACHNER .....36 Aequidens

EIGENMANN et BraY .115 Aeschrischthys .....77 Aeschynichthys .....173 Aesopia ...........169 Aethaloperca ......93 Aethoprora .........31 Aetiasis ...........105 Aetobatis ...........16 Aetobatus ..........16 Aetomylaeus ........16 Aetoplatea ..........16 Afronandus ........115 Agalaxias .........26 Agamyxis ........57 Ageneiogarra ......45 Ageneiosus ..........57 Ageniogobio .......45 Agenor ..........112 Aglyptosternon .....66 Agmus ..........6.63 Agnathomyzon .......7 Agnus ............126 Agoniates ............36 Agonocottus .......160 Agonomalus .......162 Agonopsis ..........162 Agonostomus .......77 Agonus ............162 Agosia ..........45 Agrammus .........158 Agriopopa ........119 Agriopus ..........159 Agriosphyraena .....77 Agrostichthys ......87 Agunia ............146 Ahlia ............70 Ahynnodontophis ...666 Aida .............76 Aidablennius .......127 Aidaprora ........76 Ailia ...........6.60 Ailichthys .......66 Ailurichthys ........56 Ainocottus ..........161 Ainosus ..........146 Akamefugu ..........34 Akysis ..........60 Alabes ...........72 Alaeops ............167 Alausa ............21 Alausella .........21 Alazon ...........81 Albacora ..........140 Albatrossia .......84 Albula OsBeck ......25
Albula Gronow .......19

Albula CATESBY ......77

Albula SCOPOLI ......19

Albula B. et S. .......19

Albulichthys .......45

Alburnellus ........45

Alburnoides .......45

Alburnops ........45

Alburnus CATESBY ...108

Alburnus RAFINESQUE .45

Alcichthys ........161

Alcidea ............ 160

Alcockella ........20

Alcockia ............134

Aldrichetta .......777

Aldrovandia .......73

Alectis ............102

Alectrias ...........131

Alectridium ........131

Aledon ..............36

Aleisterius ....... 138

Alepes .............102

Alepideleotris ......144

Alepidogobius .......146

Alepidomus ........77

Alepidosaurus ............

Alepisaurus .........32

Alepocephalus .....20

Aleposomus GILL ....20

Aleposomus Roule ...20

Alestes ...............37

Alestiops ............37

Alestobrycon .......37

Alestogrammus .....37

Alestopetersius .......37

Aleuteres ..........138

Alexeterion .........134

Alexurus ............144

Alfaro ............81

Algansea .........45

Algoa ..............85

Algoma ..........45

Allabenchelys ......61

Allanetta ..........77

Allartedius ...........160

Allector ...........173

Allenina ..........4.

Allinectes ...........64

Allochela ..........45

Allochir ...........164

Alloclinus .........129

Alloconger .......68

Allocottus .........161

Allocyttus .........90

Allodanio .........45

Allodontichthys .....80

Allodontium .......81

Allogambusia ......81

Allogobius ..........146

Alloheterandria .....81 
Alloleps ............133 Allolumpenus ........131 Allomonacanthus ....138 Allomycterus ........35 Alloophorus ........80 Allophallus .........79 Allopoecilia ..........81 Alloricuzenius ........160 Allosebastes ........154 Allosmerus .........25 Allosomus .........25 Allotaius ...........111 Allothunnus ........140 Allotis ............96 Allotoca ..........80 Allouarnia .........30 Allurus ...........164 Alopecias ............99 Alopias ...........99 Alosa LincK ........21 Alosa Cuvier .......21 Alphestes ..........93 Alpismaris ..........30 Alticops ..........127 Alticus (C.) LACÉP. . . 127 Alticus (C.) BLKR. ..127 Altigena .........45 Altipoma ..........139 Altiserranus ........991 Altona ...........43 Alutarius ................... Alutera ..........138 Aluterius .........139 Aluterus ..........139 Alvarius ..........99 Alvordius .........999 Alysia .............31 Amacrodon ........172 Amanses ..........139 Amaralia .........6.63 Amarginops ........58 Amate .............168 Ambassis . . ........97 Amblodon ......44-108 Ambloplites ........96 Amblyapistus ......154 Amblyceps .......660 Amblychaeturichthys .146 Amblycirrhitus ......118 Amblydoras .........57 Amblyeleotris .......144 Amblygaster ........21 Amblyglyphidodon ...119 Amblygobius ......146 Amblyopsis ........76 Amblyopus .........152 Amblyotrypauchen ...152 Amblypharyngodon ...45 Amblypomacentrus ...119 Amblyraja
Amblyrhynchichthys ..45 Amblyrhynchotus ....35 Amblyscion ........108 Amblystilbe .........37 Amblytoxotes .......113 Ameiurus ..........59 Amentum .........22 Amia Linné ........19 Amia Gronow $1763 \ldots 98$ Amia Gronow $1854 \ldots 98$ Amia Browne .......19 Amiatus .............19 Amiichthys ........99 Amioides ..........98 Amitra ...........164 Amitrichthys .......164 Amiurus ...........59 Amma ...........146 Ammocoetes ........7 Ammocoetus ........7 Ammocrypta .......999 Ammodytes .........136 Ammodytoides ......136 Ammopleurops ......169 Ammotretis .........168 Amneris ..........76 Amniataba ...........108 Amora ............158 Amorphocephalus ....122 Amoya ............146 Ainphacanthus .....13? Ampheces .........122 Amphelikturus .....82 Amphibichthys .....173 Amphichthys ........152 Amphigonopterus ...119 Amphilius ........60 Amphilophus ........116 Amphiodon ........20 Amphipnous .......72 Amphiprion ........119 Amphiprionichthys ...157 Amphiscarus .......123 Amphisile ........73 Amphisilen ........74 Amphistichus .......119 Amphisyle ........74 Amphitherapon ......108 Amphotistius ........16 Amplolabrius ......45 Amplova J. et S. ....24 Amplova W. .......24 Anabarilius .......45 Anabas ...........143 Anableps Gronow ...81 Anableps (G.) ScoP. . .82 Anableps (G.)

BL. et SCHDR. . ...82 Aanacanthobatis .....16 Anacanthus (Eh.) Cuv. .16
Anacanthus GRAY ....139

Anacyrtus ............37

Anadoras ..........57

Anago ............68

Anagramma ...........

Analithis ..........15

Anampses .........122

Anarchias ........66

Anarchichas .......129

Anarchopterus .......82

Anarhichas .......129

Anarmostus . ........107

Anarrhichas .......129

Anarrhichthys ......132

Anastomus ........41

Anathyridium ... ...168

Anatirostrum .......146

Anatolichthys .......79

Ancharius .........56

Anchichoerops .....121

Anchisomus ........34

Anchoa ...........24

Anchovia .........24

Anchovicypris ......45

Anchoviella ........24

Ancistrus ..........65

Ancylodon .........101

Ancylopsetta .......166

Ancylostylos .......21

Andameleotris .......145

Andamia ..........130

Andersonia .......60

Andracanthus ......129

Andreasenius .......79

Anema ...........126

Anemanotus ........56

Anematichthys .....45

Anemoces ..........99

Anemura ...........106

Anepistomon .......70

Angelischthys .......114

Angola ............21

Anguilla ..........66

Anguillichthys .....6.67

Anguisurus .......70

Aniculerosa .........159

Anisarchus ........131

Anisistia ........42

Anisocentrus ......76

Anisochaetodon ....114

Anisochirus ........169

Anisochromis ......93

Anisotremus ........107

Annamia .........55

Anoara ............158

Anodontoglanis ......57

Anodontostoma .....23

Anodus ............41

Anogramma ........91

Anomalodon ........107

ANN. PARASITO. HUM. ET COMP., 1962, 37, $\mathrm{n}^{\circ} \mathbf{6}^{\mathrm{bis}}$ (fasc. suppl.) 


\begin{tabular}{|c|c|c|}
\hline Anomalophryne & Aphododerus & \\
\hline Anomalops & Aphoristia . & Apostasella \\
\hline Anomalopterichthys & Aphos ... & Apostasis \\
\hline Anomalopterus .... & Aphredoderus & Apostata ............ \\
\hline Anoplagonus .......163 & Aphritis & Aprion \\
\hline Anoplarchus ...... & phrodedirus & Aprion \\
\hline Anopleutropius ... & Aphthalmichthys .. & Aprionodon \\
\hline Anoplocapros ..... & Aphyocharax .... & Apristurus ....... \\
\hline Anoplogaster .......89 & Aphyocheirodon ... & Aprodon ............ \\
\hline Anoplopoma . .....158 & Aphyocypris ..... & Aprolepis \\
\hline Anoplopetrus .......60 & Aphyocyproides .....45 & 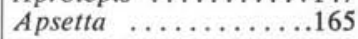 \\
\hline plus ..........110 & Aphyodite ....... & Apseudobranchus .. \\
\hline$\ldots \ldots \ldots 7$ & Aphyogobius ..... & cephalus ... \\
\hline tichthys ...... & Aphyonus & .105 \\
\hline$u s \ldots \ldots \ldots$ & semion $\ldots$. & richte ........... \\
\hline omoides ..... & Apionichthys ....... 168 & chthys $\ldots$ \\
\hline omos & Apiorhynchus ......45 & Apterigia ............72 \\
\hline nus (G.) SCOP. .41 & $a \ldots \ldots$. & Apterogasterus .......22 \\
\hline mus Cuv. Ок. ..41 & $s \ldots \ldots \ldots \ldots 154$ & ronotus. \\
\hline terus ..........29 & gramma $\ldots .37-116$ & urus. \\
\hline ia WARREN ... & ann. & Apterygia \\
\hline gia Boulgr. .. & phis . & Apterygoc \\
\hline giichthys $\ldots \ldots$. & .96 & .163 \\
\hline$\ldots \ldots \ldots$ & .153 & $\begin{array}{l}\text { Aptocyctus } \ldots . . . . \\
\text { Aptychotrema } \ldots\end{array}$ \\
\hline himaera ......18 & Apl & \\
\hline ablennius .....127 & .159 & .34 \\
\hline rius $\quad \ldots \ldots \ldots 170$ & oma ..... & $\begin{array}{l}\text { Apureaoras } \ldots \ldots \ldots \ldots \\
\text { Aracana } \ldots \ldots \ldots \ldots\end{array}$ \\
\hline atus $\ldots \ldots \ldots$ & 159 & Aracanostracion ......33 \\
\hline ......... & is $\ldots .$. & 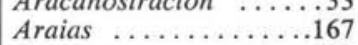 \\
\hline . $\ldots \ldots \ldots \ldots 91$ & chthys... & $\begin{array}{l}\text { Arrasas } \\
\text { Aramaca } \ldots \ldots \ldots \ldots \ldots 16\end{array}$ \\
\hline icus $\quad . . \ldots . . .991$ & $s \quad \ldots \ldots$ & $\begin{array}{l}\text { Aramaca } \\
\text { Arapaima }\end{array}$ \\
\hline arus ........165 & .26 & \\
\hline (n...... & lus ..... & ciosa $\ldots \ldots \ldots \ldots$ \\
\hline imaera .... & notus & amia $\ldots \ldots \ldots \ldots$ \\
\hline$\ldots \ldots \ldots$ & on ..... & 160 \\
\hline ocottus .... & 141 & 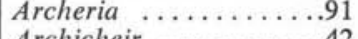 \\
\hline anc..... & 45 & $\ldots .$. \\
\hline tius $\ldots . .$. & es (O.) & … \\
\hline rodon ........ & 152 & enidia .. \\
\hline$\ldots \ldots \ldots$ & et $\mathrm{V}$. & erca $\ldots .$. \\
\hline & thys ... & plites \\
\hline hys & don. & rgus \\
\hline$\ldots$ & $\operatorname{nax}$. & scion ..... \\
\hline hys & & $A$ \\
\hline don & dia & dus \\
\hline$i s \ldots \ldots \ldots$ & lanis & $i j a$. \\
\hline & & copus. \\
\hline & & enus \\
\hline on $\ldots$. & hyoides & biscis \\
\hline & hys & \\
\hline & & scus \\
\hline canthus .. & ps $\ldots \ldots \ldots$. & A \\
\hline$\ldots \ldots$ & & ithys \\
\hline & & bius \\
\hline opus $\ldots$ & BENT. & Argentina \\
\hline 106 & thys & Arges \\
\hline thys & Ila & Argo \\
\hline Aphelikturus ....... & Apol & Argonectes .... \\
\hline & $\cos$ & Argopleura \\
\hline & & \\
\hline
\end{tabular}


Argyctius ...........8.87 Anomiolepis .........108 Argylepes ............ Argyrea ...........77 Argyreiosus .......102 Argyreus ........45 Argyripnus ........27 Argyrlepes ..........106 Argyrocottus .......161 Argyropelecus .......27 Argyrops .........111 Argyrosomus PYL. . . 108 Argyrosomus Agz. ....25 Argyrotaenia .......136 Argyrozona ........111 Arhynchobatis .......15 Ariodes .......... 56 Ariomma ............143 Ariopsis GILL .......56 Ariopsis PEYER ......56 Ariosoma .........69

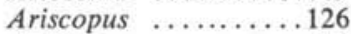
Aristeus .........76 Aristichthys .......45 Aristichromis ......116 Aristommata ........65 Aristostomias ......28 Arius ............56 Arizonichthys ......79 Arlina ...........999 Armatogobio .......45 Arndha ...........66

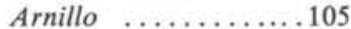
Arnion .............77 Arnoglossus .........165 Arnoldichthys .......37 Arotrolepis ..........139 Arothron ..........34 Arrhamphus ........74 Arripis ...........105 Artedidraco .........126 Artedielloides .......160 Artediellus ........160 Artedius ...........160 Arthrobatis ........15 Arthrophallus ......81 Arthropterus .......15 Artisia ...........121 Aruma ........... 147 Arusetta ...........114 Ascelichthys .......162 Asellus

(P.) LACPD. . . . . 145

Asellus VAL. .......86 Aseraggodes .........168 Asiphonichthys ......37 Askoldia .........132 Aspasma ..........153 Aspasmagaster .......153
Aspasmichthys

Aspasmodes ............153 Asper .............100 Aspericorvina ...... 108 Asperina ...........108 Asperulus SchäFER . . 100 Asperulus KLEIN .....100 Asperulus (K.) GILL ..100 Aspicottus ........160 Aspidobagrus .......58 Aspidontus ........ 127 Aspidoparia ........45 Aspidophoroides .....163 Aspidophorus .....58-163 Aspidoras ..........65 Aspiobarbus ........45 Aspiolucius ........45 Aspiopsis ..........45 Aspiorhynchus .....45 Aspiscis ..........998 Aspistor .........556 Aspisurus .........137 Aspitrigla .......... 156 Aspiurochilus ......121 Aspius ..........45 Aspredinichthus ....663 Aspredinichthys ....663 Aspredo GRONOW ....663 Aspredo (G.) Scop. ....63 Aspro Cuv. et VAL. . . 100 Aspro (C.) LACEPD. . . . 998 Asprocottus ........162 Asproperca ..........999 Asprotilapia ........ i16 Asquamiceps ........20 Asra ..................... Assesor ..........96

Assiculus ........996 Assurger ..........141 Astatheros ........116 Astatichthys .......999 Astatochromis ......116 Astatore ..........116 Astatoreochromis ...116 Astatotilapia ........116 Astea ............... Astemomycterus ....664 Asternopteryx ......132 Asternotremia ......73 Asterophysus .......57 Asterorhombus ......165 Asterotheca ........ 163 Asterropteryx .......145 Asthenurus ........85 Astrabe ............ 147 Astrape ........... 17 Astrapogon ........98 Astroblepus ........64 Astrocanthus .......171 Astroconger ........69
Astrocottus ....... 160

Astrodoras .........57

Astrodermus .......990

Astrolytes ........ 160

Astronesthes .......27

Astronotus .........116

Astroscopus ........126

Astyacinus ...................

Astyanax ..................

Asymbolus ......... 12

Ataeniobius ........80

Atahua ...........110

Ateleobrachium ......84

Ateleopus .......... 32

Atelomycterus .......12

Atelurus ..........82

Atheresthes .................. 167

Atherina ..........77

Atherinason ........77

Atherinella .........77

Atherinichthys ......77

Atherinoides .......77

Atherinomorus .....77

Atherinops .........77

Atherinopsis .......77

Atherinosoma ......76

Atherion ..........77

Atherodon .........84

Atherthyring .......77

Athlennes .........74

Atimostoma ......... 143

Atomaster .........41

Atopichthys .......69

Atopochilus .......61

Atopoclinus ........131

Atopocottus ........ 160

Atopomesus ...........37

Atopomycterus ......35

Atractoperca .......91

Atractophorus .......13

Atractoscion ........101

Atractosteus .........19

Atrilinea ..........446

Atrophacanthus .....138

Atropus ..........102

Atrosalarias .........127

Attilus ............108

Atule JoRD. ........102

Atule Nichls. ...... 102

Atulonotus ...........137

Atuona ............................

Aturius ..........46

Atypichthys ....... 112

Atyposoma ........112

Atypus ............112

Auchenalepoceps ....20

Auchenaspis .......58

Auchenionchus ......129

Auchenipterichthys ...57 
Auchenipterus

Cuv. et Val. .....57

Auchenipterus GTHR. .129

Auchenistius ........131

Auchenoceros ......85

Auchenoglanis .......58

Auchenopterus ......129

Aulacocephalus ......93

Aulichthys .........76

Auliscops ..........76

Aulixidens ..........37

Aulohalaelurus ......12

Aulohoplostethus ......88

Aulonocara REgAN ...116

Aulonocara

Trewavas ........116

Aulonocranus ......116

Aulopareia ..........147

Aulophallus ........81

Aulopus ...........30

Aulopyge .........46

Aulorhynchus ......76

Aulostoma ........7.73

Aulostomatomorpha ...20

Aulostomus .........73

Aulotrachichthys .....88

Aulus ...........73

Aurata CATEsBy .....111

Aurata (Cuv.) Ок. ...111

Aurata Risso ........111

Aurigequula ........106

Aurigobius .........147

Aurion ...........126

Ausonia ..........142

Australaphia .......147

Australuzza ........77

Austranchovia ......24

Austroatherina .....78

Austrobatrachus ....152

Austroberyx ........88

Austrochanda ......997

Austrocobitis .......26

Austrofundulus ......79

Austroglossus . ...168-169

Austrogobio . . . .....147

Austrogobius .......147

Austrolabrus .......122

Austrolethops ......147

Austrolichthys ......133

Austrolicichthys .........

Austrolycus ........133

Austromenidia ......78

Austropagrus .......111

Austroperca .........999

Austrophycis .......85

Austrosparus ........111

Austrotirus ...........30

Autanadoras .......57

Autanichthys ........37

Autisthes .........108
Auxis ............140

Averruncus .......163

Avocettina ........72

Avocettinops .......71

Awaous ...........147

Axineceps .........111

Axinurus .........137

Axoclinus ..........129

Axvrias ............160

Aygula ............122

Aylopon ...........91

Ayresia ............119

Azevia ................65

Azteca ..........46

Aztecula .........46

A zuma ............ 132

Azurella ..........119

Azurina ............119

Azygopus . . .......168

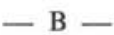

Badis ...............115

Baeostoma ..........168

Bagarius ...........60

Bagre Catesby ......59

Bagre (Cuv.) Ок. .....56

Bagrichthys ........58

Bagroides ..........58

Bagropsis . .........62

Bagrus ...........58

Bahaba ...........101

Baicalocottus ........162

Baillonus ..........102

Baiodon ...........116

Baione ...........24

Baiostoma .......... 168

Bairdiella ........... 108

Bajacalifornia ......20

Balantiocheilos ......46

Balistapus ..........138

Balistes .............138

Balistoides ............138

Balitora ............55

Balitoropsis ........55

Ballerus ...........46

Balsadichthys ......80

Bambradon .........158

Bangana .........46

Banjos .................110

Barathrites ..........134

Barathrodemus .....134

Barathronus .......134

Barbantus ........21

Barbatogobius .......147

Barbatula ..........54

Barbellion W. .......46

Barbellion Germar .....

Barbichthys .......46

Barbodes .............. 46
Barbodon ..........46

Barbopsis ..........46

Barbourichthys ......168

Barbourisia ...... 33-89

Barbulifer ..........147

Barbupeneus .......110

Barbus ...........446

Barchatus ........152

Barilius ..........46

Bario .............37

Barnardichthys .....168

Barrosia ..........96

Bartamiolus ..........96

Bartschina ........96

Barynotus .........46

Bascanichthys ......70

Bascanius ...........165

Basilichthys .......78

Basiliscartes ...........

Basilisciscartes ......127

Bassanago ........6.69

Bassetina ..........102

Bassobythites ........134

Bassogigas .........134

Bassozetus .........133

Batanga ..........145

Batasio ............60 60

Bathaploactis .......159

Bathophilus Gigliol. ..28

Bathophilus MrLes ....6 64

Bathyaethiops .......37

Bathyagonus ........163

Bathyalopex .......18

Bathyanthias ........91

Bathyaploactis ......159

Bathybates .........116

Bathybatrachus .....152

Bathyceratias .......172

Bathyclarias .......66

Bathyclupea .........75

Bathycongrus ......6.69

Bathyairaco .........126

Bathyembryx .......28

Bathygadus ..........84

Bathygobius .......147

Bathylaco ...........30

Bathylagoides ........26

Bathylagus ..........26

Bathylychnops ......26

Bathylychnus .... 27

Bathymacrops .....26-26

Bathymaster ........125

Bathymicrops ........31

Bathymyrus ........70

Bathymyzon ........7

Bathynectes .........134

Bathyonus ........134

Bathyophis ....... 27

Bathypercis

ALCOCK $1880 \ldots 125$ 
Bathypercis

ALCOCK 1893

Bathyphasma .......124

Bathyphylax ..........138

Bathypropteron .......20

Bathypterois .........32

Bathypygidium .....6.64

Bathyraja ...........15

Bathysaurops .....30-31

Bathysauropsis ........31

Bathysaurus .........30

Bathysebastes .......154

Bathyseriola ........143

Bathyphaera .......28

Bathysidus ...........33

Bathysolea ..........168

Bathysphyraenops ....998

Bathystethus ........142

Bathystoma SCUDDER .107

Bathystoma FITZGER ...46

Bathystorreus ......134

Bathysudis ..........31

Bathythrissa ........20

Bathytoshia ........16

Bathytroctes .......20

Bathytyphlops ........31

Bathyuroconger .....6.69

Batis .............15

Batman .........147

Batracheleotris .....145

Batrachocephalus

BLKR. $1847 \ldots \ldots .56$

Batrachocephalus

BLKR. $1858 \ldots \ldots .56$

Batrachocottus ........162

Batrachoglanis ......62

Batrachoides ........152

Batrachomoeus ......152

Batrachops HeCKEL ...116

Batrachops BIBRON ....35

Batrachopus ........171

Batrachus KLEIN ...170

Batrachus

BL. et SCHNR. ....152

Batrichthys .........152

Batrictius .........152

Bayonia ...........116

Bdellorrhynchus ....170

Bdellostoma ...........7

Beanea ............88

Beaufortella ...........168

Beaufortia ..........55

Bedotia ...........76

Bedula ............115

Beirabarbus .........46

Bellator ............156

Belligobio .........46

Bellocia .............20

Bellottia ..........134

Belobranchus ........145
Belodonichthys .....58 | Biotaecus ..........116

Beloholocentrus .....88 Biotodoma .........116

Belone ............74 Bipinnula ...........141

Belonesox .........81 Bitricarinata .......41

Belonichthys .......82 Bivibranchia ........37

Belonocharax ......41 Biwia ..........46

Belonochromis ......119 Blakea ...........129

Belonoglanis ......6.60 Blanchardia ..........134

Belonoperca .......991 Blandowskiella .....995

Belonophago .......43 Blandowskius ........138

Belonopsis ........72 Blavania ..........55

Belonopterois ........32 Bleekeria CASTLN. ...96

Belonopterygion .....96 Bleekeria GNTHR. ...136

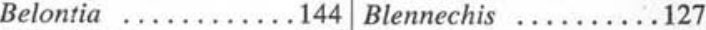

Belophlox ..........100 Blennicottus ...........161

Bembradium .......158 Blenniella ..........127

Bembras ...........158 Blennioclinus ......129

Bembrops .........125 Blenniolus ........128

Bendilisis .........46 Blenniomimus ......129

Bengala ..........46 Blenniops ..........132

Bengalichthys ......17 Blennitrachus .....128

Bennettia ..........105 Blennius .............128

Bentenia ..........91 Blennodesmus .......132

Benthalbella ........28 Blennophidium ......132

Benthenchelys .....66 Blennophis SwaIns ...129

Benthobatis .........17 Blennophis VAL. ....128

Benthocometes ......134 Blennus ...........128

Benthodesmus .......141 Blepharichthys .....102

Benthophiloides .....147 Blepharis ...........102

Benthophilus ........147 Blepsias ...........160

Benthosaurus ........33 Bleptonema .........37

Benthoscopus .........126 Blicca .............46

Benthosema ..........31 Bliccopsis .........46

Benthosphyraena .....20 Bodianus BLOCH ..93-121

Bergia ............37 Bodianus CUVIER ..93-121

Bergiaria ........6. Boesemanichthys .....34

Bergiella .........62 Boggiania ...........116

Beridia ........................ Bogimba

Bermudichthys ......122 Bogmarus ........87

Bero .....................97

Berowa ...........147 Bogoslovius ........ 84

Bertelsenna .........172 Bogota ...........97

Bertinichthys .......46 Bola HaMILT. ........108

Bertinius .........46 Bola GNTHR. .......46

Bertinulus ........72 Bolbometopon .......123

Bertoniolus .........37 Boleichthys .........99

Beryx .......... 88 Boleophthalmus ..... 152

Besnardia ....................... Bolep2

Betta ............143 Boleosoma .........99

Bhanotia .........82 Bollmannia ........ 147

Bhanotichthys ......82 Bombonia ........82

Bhavania .........55 Bonapartia ........26

Biat ........................41

Bibronia ................. Bondichthys ......

Bidenichthys .......134 Boops GRoNow ......97

Bidyanus ..........100 Boops CUVIER .......113

Bikinigobius .......147 Boopsetta ............165

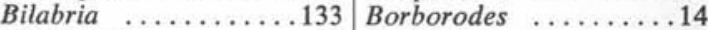

Binghamia .........20 Borborys ..........79

Binghamichthys .....20 Boreocottus .......161 


\begin{tabular}{|c|c|c|}
\hline ogadus & chymesistius & Brunichthys \\
\hline Boreogaleus ... & Brachymullus .... & Brustiarius ... \\
\hline Boreogobius . & Brachymystax $\ldots$ & Brvanina \\
\hline preophryne .. & Brachymystus ........28 & Bryaninops \\
\hline Boridia .......... & Brachynectes .......128 & Brycinus \\
\hline Boroda & Brachyochirus .... & Brycochandus \\
\hline Borodamirus . & Brachyonichthys .. & Brycon \\
\hline Borodinula ...... & Brachyopsis .........163 & Bryconacidnus .... \\
\hline Borostomias $\ldots \ldots$ & Brachyoptera ....... & Bryconaethiops ..... \\
\hline Bostockia ..... & Brachypetersius ... & Bryconalestes ....... \\
\hline Bostrichthys ....... & Brachyplatysoma ......62 & Bryconamericus .......37 \\
\hline Bostrictis .......... & Brachypleura ..... & Bryconodon .........37 \\
\hline Bostrychoides .... & Brachypleurops .... & Bryconops ......... \\
\hline Bostrychus ..........145 & Brachypomacentrus ..119 & Bryolophus ........132 \\
\hline agonus $\ldots \ldots \ldots 163$ & Brachyprosopon ......167 & emma ........ \\
\hline Botrocara & Brachypterois ... & Bryozoichthys ... \\
\hline Bothrocarichthys .. & Brachyrhamdia .. & Bryssetaeres ...... \\
\hline Botrocarina $\ldots \ldots \ldots 133$ & amphichthys ..43 & Bryssophilus ...... \\
\hline Bothrocaropsis .... & Brachyrhaphis ......81 & Bryttosus \\
\hline Bothrolaemus .... & Brachyrhinus .......991 & Bryttus \\
\hline Bothus $\ldots \ldots \ldots \ldots 165$ & yrus $\ldots \ldots \ldots 154-169$ & Bryx \\
\hline Botia & Brachysc & alichthys \\
\hline gerella .... & Brachysynodontis .. & Bubyr ..... \\
\hline gerina ..... & Brama KLEIN .......46 & $B u$ \\
\hline Boulengerochromis ...116 & Brama (K.) BLKR. ... & \\
\hline htus .........126 & $a$ BL. et SHDR. . . .990 & hthys \\
\hline thys $\ldots \ldots \ldots 126$ & ichthys $\ldots \ldots \ldots 113$ & ratias $\ldots .$. \\
\hline ctus $\ldots . . . \ldots$. & Bramocharax ....... & sidium . \\
\hline gla $\ldots$ & .119 & .168 \\
\hline Bowenia ..... & .102 & naricus .... \\
\hline Bowersia ......... & stegus RAFSQ. .101 & Bunaka ...... \\
\hline$\ldots \ldots \ldots$ & tegus GILL ..58 & \\
\hline 104 & $c a \ldots \ldots \ldots 64$ & phalichthys \\
\hline aelurus... & $\mathrm{Br}$ & phalus ... \\
\hline teres ...... & .128 & ttus. \\
\hline chthys ... & os $\ldots$. & thalmus \\
\hline optera $\ldots . .$. & einia $\ldots \ldots \ldots 60$ & $\ldots \ldots$ \\
\hline tilon $\ldots . . .$. & $a \ldots \ldots$. & bulla . \\
\hline . . . . 154-169 & $m a \ldots$ & 137 \\
\hline estes $\ldots . . .$. & .56 & nga . \\
\hline blyopus ....152 & nis $\ldots . .$. & obius \\
\hline abras .... & bio $\ldots . .$. . & 144 \\
\hline allionymus $\ldots 127$ & $\ldots \ldots 105$ & Butyrinus \\
\hline alus & & Bythites \\
\hline cinus & & \\
\hline heirophis $\ldots \ldots 70$ & innella .. & $\mathrm{C}$ \\
\hline gger BLKR. & $a \ldots \ldots$ & \\
\hline ger NORMAN. & & Cabdio \\
\hline yconger REGAN ..70 & Brockius .. & illus \\
\hline nio $\ldots \ldots \ldots 46$ & Brontes. & \\
\hline iterus & & ichthys \\
\hline eleotris .......145 & culus & Cabotina .. \\
\hline dus ... & & \\
\hline laxias & phycis & \\
\hline genys $\ldots . . . \ldots 107$ & Brosmus & Cacodoxus \\
\hline anis $\ldots$. & & Cacumen \\
\hline ucosome & $B r$ & \\
\hline bius .... & 134 & Caecoharbus \\
\hline$n a$ & & \\
\hline & & \\
\hline
\end{tabular}


Caecorhamdia ......62

Caecula ...........70

Caenotropus .........41

Caenthigaster ..........33

Cnesio ..........105-107

Caesiomorus .......102

Caesioperca .........91

Caesioscorpis ........105

Caesiosoma ........112

Caeso ..............111

Caffrogobius ........147

Cainosilurus ........57

Calamiana .........147

Calamoichthys .....173

Calamuroena .......70

Calamus ............111

Calcarbrotula ......134

Calcarobrotula ......134

Callanthias .........91

Callarias ..........85

Callaus ...........108

Callechelys .........70

Calleleotris ..........145

Calliblennius

BARBOUR .........129

Calliblennius AOYAgI .128

Callicanthus .......137

Callichthys GRONOw ..65

Callichthys (G.) Scop. .65

Callichrous .........58

Calliclinus .........129

Callidulus .........91

Callieleotris ........145

Callimucenus .......127

Calliodon

BL. et SCHNDR. . . ...123

Calliodon Cuv. .......123

Calliomorus ........158

Callionymus ........127

Calliscyllium .........11

Calliurichthys ......127

Calliurus ..........96

Callochromis .......116

Callogobius .........145

Callomystax ......66

Callepanchax ......79

Callopharynx ........116

Callophysus ........62

Calloplesiops .........96

Calloptilum

RICHARDSON .......85

Calloptilum Hutron ...85

Callorhynchus

Gronow ........18

Callorhynchus

(G.) Cuv. .......18

Callosphyraena ......77

Callyodon Gronow ..124

Callyodon (G.) Scop. .124

Callyogobius
Calophysus

Calopomus

Calotomus

.62

Calumia .............145

Calycilepidotus .....160

Calymmichthys ......127

Camarina ..........113

Cambellina .........88

Campichthys ........82

Campogramma .....102

Camposella ........42

Camposichthys

Travas. ........... 37

Camposichthys WHITY .42

Campostoma .......46

Campylodon ........73

Campylomormyrus ...29

Candidia ..........46

Caninoa ............13

Caninotus .............13

Cannorhynchus ....73

Cantharus .........113

Cantharusa ...........113

Cantherines .........139

Canthidermis ........138

Canthigaster .........34

Canthirhynchus ......163

Canthophrys .......54

Canthopomus ......6.65

Cantoria ............169

Cantorusia ..........169

Capartella ...........168

Capellaria ..........171

Capoeta ..........46

Capoetobrama .......46

Caprichthys ..........33

Capriscus KI.EIN ....138

Capriscus Röse ......138

Capriscus RaFnsQ. ...138

Capriscus SwaIns ....138

Caprodon ...........91

Capromimus ........990

Caprophonus .......990

Capropygia ..........33

Capros ............90

Caprupeneus ........110

Caquetaia ...........116

Caracanthus ..........157

Caragobioides ........147

Caragobius .........147

Caragola ..............7

Caralophia ........70

Caranactis ........172

Carangichthys .......102

Carangola ...........7

Carangoides ........102

Carangops .........102

Carangulus .........102

Carangus Agz ........102

Carangus GIRARD ....102
Caranthus .........113

Caranx ............102

Caranxomorus ......991

Carapo ............43

Carapus RAFNSQ. . 44-136

Carapus

(Cuv.) Mül. Trosch.

Carapius................ 43

Carassioides .........46

Carassiops ..........145

Carassius ..........46

Carcharhinus ........11

Carcharias RAFNSQ. ...10

Carcharias GISTEL . ...13

Carcharias Cuv. .......11

Carcharocles .........10

Carcharodon .........10

Cardiopharynx ......116

Careliparis ..........164

Carelophus .........132

Caremitra ...........164

Careproctus ...........164

Caribrhegma ........91

Cariburus ...........84

Carinotetraodon .....34

Carinotus .........6.65

Caristioides ........143

Caristius ..........88

Carlana ..............37

Carlhubbsia ........79

Carlia ..............37

Carnegiella ........41

Carpio S.D.W. .....44

Carpio HeCKel ......46

Carpiodes .........44 44

Carpionichthys .......46

Cascadura ........6.65

Caspialosa ........22

Caspiomyzon .........7

Caspiosoma .........147

Cassigobius ........ 147

Castelnauina .......82

Catabasis ............37

Catablemella ........31

Cataety ..............134

Catalufa ...........98

Cataphractops ......65

Cataphractus KLEIN. . . 163

Cataphractus

(K.) Flemg. . . . . . 163

Cataphractus

GRonow ..........165

Cataphractus BLOCH ...65

Cataphractus CATESBY .57

Catastoma ..........56

Catathyridium .......168

Cathetostoma .......126

Cathorops .........56

Catla ............46 
Catlocarpio ........46

Catochaenum .......109

Catonotus ...........999

Catophorhynchus .....35

Catopra ..........115

Catroprion ...........37

Catostomus ........44

Catulus ValmT. ........12

Catulus Sмттн .......12

Catulus (V.) Garm. ...12

Caudania ..........20

Caularchus ..........153

Caulichthys ........145

Caulistius ..........153

Caulolatilus ...........101

Caulalepis ........89

Caulophryne .......172

Caulopsetta ........165

Caulopus ...........32

Cauque ...........78

Cavelampus .........31

Cayennia ..........152

Cebedichthys .......131

Cebidichthys ........131

Celema ............134

Cenisophius .........46

Centaurus .......... 36

Centracantha .......112

Centracanthus .......112

Centracantus .......112

Centracion ..........9

Centranodon ........158

Centratherina .......76

Centrarchops .......95

Centrarchus Cuv. ....996

Centrarchus

Jodn. et Evmn. . ....96

Centridermichthys ...161

Centrina ............13

Centriscops ........74

Centriscus ..........74

Centristhmus ........93

Centroberyx .........88

Centroblennius .......131

Centrobranchus .......31

Centrocetus ........172

Centrochir .........57

Centrochromis ......120

Centrodoras .........57

Centrodraco .........127

Centrogaster .........137

Centrogenys ........93

Centrogobius ........147

Centrolabrus .......121

Centrolophodes ......142

Centrolophus ........142

Centromochlus .....57-58

Centronotus LACPD. ...102

Centronotus

BL. et SHNDR.
Centropercis .......126

Centropholis .......91

Centropholoides .....91

Centrophoroides .....13

Centrophorus

Müll-Henl. . . . . . 13

Centrophorus KNER ...42

Centropodus ........112

Centropogon .......154

Centropomus ........95

Centropristes .......91

Centropristis .......91

Centropus ..........157

Centropyge .........114

Centroscyllium ........13

Centroscymnus .......13

Centroselachus ........13

Centruriphis ........70

Cephacandia ........164

Cephalacanthus ......164

Cephalaspis ........87

Cephalepis .........87

Cephaleutherus ......15

Cephalinus .........159

Cephalocassis ........56

Cephalocottus .......161

Cephalofarer ........88

Cephalogobius .....147

Cephalokompsus ......46

Cephalopholis ......993

Cephalopsis FITzGr. . . .46

Cephalopsis RAFsQ. ...35

Cephaloptera ........17

Cephalopterus .......17

Cephaloscyllium .....12

Cephalosilurus ......62

Cephalurus ..........12

Cephalus BPTE. ......46

Cephalus ScHaw .....36

Cephalus (PLM.) .....77

LACEPD. .........77

Cephalus BasilwK. ....46

Cephimmus .........100

Cephus ............85

Cepola ............118

Cepolophis .........135

Ceratacanthus ......139

Ceratias ...........172

Ceratichthys

BD. et GIRD. . .....46

Ceratichthys GIRD. ....46

Ceraticthys ........46

Ceratobatis ............... 17

Ceratobranchia .......37

Ceratocaulaphryne ...172

Ceratocheilus .........57

Ceratocottus ........160

Ceratoglanis ........58

Ceratoptera ..........17

Ceratothrynchus ........57
Ceratostethus .......76

Cerdale ............132

Cerdo .............85

Cerictius ............13

Ceris ..............137

Cerna .............93

Cernua SchFr. ........100

Cernua Flmg. ........100

Ceromitus ..........72

Cestracion KLeIN .....99

Cestracion Cuv. ......9

Cestracion (K.) GILL . . 12

Cestracion (W.) Ogly. 12

Cestraeus ..........777

Cestreus KLEIN .......77

Cestreus MCCLld. ...145

Cestreus Gronow .....101

Cestrorhinus ........12

Cetengraulis ........24

Cetomimoides ......89

Cetomimus .......899

Cetonurus ..........84

Cetopsis ..........64

Cetopsogiton ......664

Cetopsorhamdia .....6.62

Cetorhinus ............9

Cetoscarus .........124

Cetostoma ........89

Chabanaudetta ......169

Chaca ............60

Chaenichthys .......126

Chaenobrythus .......96

Chaenobryttus ......997

Chaenocephalus ......126

Chaenodraco ........126

Chaenogaleus .........11

Chaenogobius .......147

Chaenomugil ........77

Chaenophryne .......172

Chaenopsetta ........166

Chaenopsis .........131

Chaenothorax ........65

Chaetichthys .......124

Chaetobranchopsis ...116

Chaetobranchus ......116

Chaetodermis .......139

Chaetodipterus .......113

Chaetodon .........114

Chaetodontoplus ....114

Chaetodontops .......114

Chaetolabrus .......116

Chaetomus ........24

Chaetopterus .........105

Chaetostoma .......116

Chaetostomus .......116

Chaturichthys .......147

Chagunius .........46

Chalaroderma .......128

Chalcalburnus ......46

Chalceus .............37 
Chalcinopelecus $\ldots \ldots .37$
Chalcinopsis ...........37
Chalcinus

Cuv. et Val. .......37

Chalcinus RAFNSQ. ....37

Chalinura ...........84

Chalinurus .........84

Chalisoma .........138

Chalixodytes .........125

Chalmina ..........84

Chamaigenes .......6.63

Champsoborus ..........37

Champsocephalus ...126

Champsochromis .....116

Champsodon .......126

Chanda ...........97

Channa Gronow ......144

Channa (G.) ScoP. . . . 144

Channalabes .......6.61

Channichthys .......126

Channomuraena ....66

Chanodichthys .......46

Chanos ............21

Chapalichthys ......80

Chaparrudo ........147

Chapinus ...........33

Characidium ......42

Characinus L. .......37

Characinus LACPD. . . . 37

Characinus Cuv. . . . . 37

Characodon ........80

Charalia ..........78

Charax Gronow ......37

Charax Risso .......111

Charaxodon .........37

Charisella ..........78

Charybdia ..........165

Chascanopsetta .....165

Chasmenchelys .....66

Chasmias ..........147

Chasmichthys ......147

Chasmistes .........44

Chasmocephalus .....62

Chasmacranus ......62

Chasmodes ........128

Chatoessus ...........23

Chatrabus ...........152

Chaudhuria .........170

Chauliodus .........28

Chaunax ...........171

Chedrus ..........46

Cheilichthys .........34

Cheilinodes .......122

Cheilinus ..........122

Cheilio ............122

Cheiliopsis .........122

Cheilobarbus .......446

Cheilobranchus ......72

Cheilodactylus ......119

Cheilodipterops .......98
Cheilodipterus .........98

Cheilolabrus ..........122

Cheilonemus .......46

Cheilopogon ........75

Cheiloprion ........120

Cheilotrema ........108

Cheimerius .........111

Cheiragonus ........163

Cheirocerus ........62

Cheirododus ........65

Cheirodon ..........37

Cheirodontops ........37

Cheirodus ..........65

Cheiromuraenesox ....66

Cheironebris .......108

Cheiroxenichthys ....105

Chela ...........46

Chelaethiops .......47

Chelidonichthys ......156

Chelidoperca ........91

Chelmo ...........114

Chelmon ..........114

Chelmonops ........114

Chelon ............77

Cheloniger ..........107

Chelonodon ...........34

Chelonodontops ......34

Chelonopsis .........36

Chenia ............91

Chenogaster .........139

Cheonda ..........47

Cherublemma .......134

Chiasmodon ........127

Chiasmodus ........127

Chicolepis .........147

Chilara ...........135

Chilatherina ........76

Chilias ...........124

Chilochromis ......116

Chiloconger ........69

Chilodipterus ........98

Chilodus .........4.42

Chiloglanis ........60

Chilogobio

BERg 1914

Chilogobio

BERG $1915 \ldots \ldots .47$

Chilomycterus .........35

Chilomyzon .......42

Chilopterus ............

Chilorhinus ........70

Chiloscyllium ........12

Chilotilapia .........116

Chimaera ............18

Chimarrhoglanis .....60

Chimarrichthys HAAST .125

Chimarrichthys SAUVG. .60

Chionodraco ........126

Chirocentrodon ......22

Chirocentrus .........21
Chirodactylus ......119

Chirodon ............37

Chirodorus ........75

Chirolophis .......132

Chirolophius .......170

Chironectes ......170-171

Chironemus .........118

Chirophryne .........172

Chiropsis .........158

Chirostoma .......78

Chirostomias .......28

Chirus .........95-158

Chitala ..........20

Chitonotus ..........160

Chlamydes .........147

Chlamydogobius ......147

Chlamydoselachus .....9

Chlevastes .........70

Chlidichthys .......96

Chloea ............147

Chloeichthys .......147

Chlopsis .........68

Chlorichthys .......122

Chlorophthalmus ......31

Chloroscombrus .....103

Chlorurus .........124

Choenorhinos ........35

Choerodonoides .......121

Choerodon .........121

Choeroichthys ......82

Choerojulis ........122

Choeroplotosus ......57

Choerops ..........121

Choeropsodes ........121

Choiridon ........121

Chologaster .........76

Chondrochilus ......47

Chondroplites .......142

Chondrorhynchus ....47

Chondrostoma ......47

Chonerhinos .........35

Chonerhinus ..........35

Chonophorus .......147

Chopinopsetta .......165

Chopraia ..........55

Choregon .........25

Choridactylodes ......157

Choridactylus ........157

Chorimycterus ......42

Chorinemus .........103

Chorismodactylus ....157

Chorismopelor ......157

Chorisochismus ......153

Chorististium .......93

Chorinorhinus ......70

Chriodorus .......74

Chriolax ..........156

Chriolepidops ..... 145

Chriolepis ........147

Chriomitra ............139 


\begin{tabular}{|c|c|c|}
\hline $\operatorname{tax}$ & ectes & glanis \\
\hline Chrionema .... & Cirriscyllium .... & Cnidon \\
\hline Chriope ..... & Cisco .......... & MUL et TSCHL. 1849.95 \\
\hline Chriopeoides ...... & Citharichthys ... & Cnidon \\
\hline Chriopeops ........79 & Citharidium $\ldots \ldots \ldots 43$ & Mul. et TsCHL. 1845.37 \\
\hline Christiella ......... & Citharinus ....... & Cobitichthys $\ldots \ldots \ldots 54$ \\
\hline Chromanthias & Citharoedus ... & Cobitiglanis ....... \\
\hline Chromichthys ... & Citharoides $\ldots .$. & Cobitinula \\
\hline Chromidotilapia ... & Citharus RösE .... & Cobitis ... \\
\hline Chromis Cuv. 1815. & Citharus REINHDT. . . . 167 & Cibitophis \\
\hline Chromis Cuv. $1817 \ldots 116$ & Citharus BLKR. .....163 & Coccia \\
\hline Chromis (C.) GüHR. ..116 & Citula ............103 & Cocciella \\
\hline LUM.) & Citularia & Coccius \\
\hline$\ldots \ldots \ldots$ & Cladoselache ...... & Coccogenia \\
\hline Chromis GRONOW ... & $\ldots \ldots \ldots \ldots 109$ & Coccolus \\
\hline Chromogobius ......147 & Cla & Coccorella ... \\
\hline Chrosomus ........47 & Clariallabes ....... & Coccotis \\
\hline hys $\ldots \ldots \ldots 59$ & Clarias Gronow ....61 & Coccotropsis. \\
\hline Chrysiptera ....... & Clarias (G.) Scop. . & Coccotropus \\
\hline Chrysobagrus ........59 & Claricola ......... & Cochlefelis .... \\
\hline blephus .......111 & Clariger ..........147 & Cochlioceps $\ldots . .$. \\
\hline Chrysomelanus .....993 & oides & Cochliodon \\
\hline Chrysoperca ........ & Clarisilurus .......58-61 & Cochlognathus. \\
\hline kekadion ....47 & hthys $\ldots \ldots \ldots 132$ & Coclonotus .... \\
\hline Chrysophris $\ldots . . .$. & Clarkina .......... & Cocotropus .... \\
\hline tosus .........87 & Clarotes ........ & Codoma. \\
\hline teryx $\ldots$. & $\ldots \ldots \ldots 88$ & -ilophis \\
\hline Chuanchic & enes ....... & Coelocephalus. \\
\hline Cibotion ..........33 & us a e & Coelonotus ...... \\
\hline Cic & .147 & phrys \\
\hline R. & $m a \ldots \ldots$ & Coelorhynchus \\
\hline CHNDR. . . . . & Climacoporus .......129 & Coelurichthys ... \\
\hline$a \ldots \ldots \ldots$ & Clinitrachus ... & Cogrus ......... \\
\hline 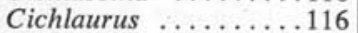 & $\ldots \ldots \ldots 161$ & $\ldots \ldots \ldots$ \\
\hline te & Clinodon .........116 & Coius .. \\
\hline Cic & Clin & ridia \\
\hline Cicla RösE ....... & 129 & axia \\
\hline Ciliata & tomus & Coleosmaris. \\
\hline Cilus & $\ldots \ldots \ldots$ & ropis ... \\
\hline . & Cliols & ........143 \\
\hline 100 & Clodalus & Coliscus ......... \\
\hline ius ... & 98 & 168 \\
\hline bus .... & 22 & .31 \\
\hline leus ........ & lodon. & Collichthys ...... \\
\hline rus ... & 22 & .90 \\
\hline$n s \quad \ldots .$. & 37 & tger \\
\hline iraena $\ldots . . . .70$ & chthys $\ldots \ldots \ldots 22$ & Colocopus \\
\hline$\ldots$. & $\operatorname{arax}$. & mmus . \\
\hline ys. & & \\
\hline$\ldots \ldots \ldots \ldots, 47$ & $\ldots \ldots 105$ & Colomesus ......... \\
\hline & Ila KessLR. ..22 & Colossoma .... \\
\hline & lla BERG. . & 156 \\
\hline ….... 118 & Clup & Colpichthys. \\
\hline hys .... & petersius & gnathus \\
\hline & .61 & Colt \\
\hline Cirrhitops . . . . . . 118 & Clupi & Columbia \\
\hline psis ... & Clypeobarbus & \\
\hline $\mathrm{C} I T$ & .160 & ndorella \\
\hline yllium..... & Cnestrodon .......881 & Compsomidiama . \\
\hline & & \\
\hline
\end{tabular}




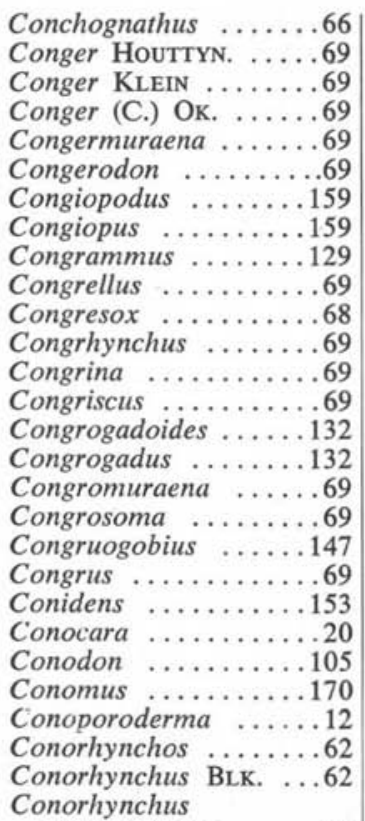

(NozmN.) Gill ....20

Conostome ........62

Conta .............58

Contusus ...........34

Cookeolus ..........91

Copeichthys .........24

Copeina ............38

Copelandellus .......999

Copelandia ........997

Copella ................38

Copidoglanis ........58

Copiodoglanis .......58

Coptodon ...........116

Coptostomabarbus ...47

Coracinus Gronow ...113

Coracinus Pallas ....108

Coradion ............114

Coraglanis .........60

Corallicola .........129

Coralliozetus ....129-130

Cordorinus ........65

Cordylus .........139

Coregoni ...........25

Coregonus .........25

Coreius ............47

Corematodus .......116

Coreobagrus ........59

Coreoleuciscus .......47

Coreoperca ........993

Coreosiniperca .......993

Coreospiniperca .......93

Corica
Coricus

Coridodax ..............

Coripareius .......47

Coris ..............122

Corizichthys ......152

Corniger .........88

Coronichthys .......153

Coronogobius ......147

Corusculus ........93

Corvina ...........108

Corvula ...........108

Corycus ..........121

Corydoras ........6.65

Corymhophanes ....665

Corynolophus ........172

Corynophorus ......172

Corynopoma ........38

Corynopomops ......38

Coryogalops ........ 145

Coryphaena ........91

Coryphaenoides .........

LACPED. ..........101

Coryphaenoides

GUNNER .........84

Coryphaesopia .....169

Coryphillus ........ 168

Coryphoblennius .....128

Coryphopterus .......147

Coryphus ..........91

Corystion KLEIN ....156

Corystion RAFNSQ. ...125

Corythobatus ....154-157

Corythoichthys .....82

Corythroichthys .......82

Coscinoxyron ........38

Cosmochilus

SAUVG. 1878

Cosmochilus

SAUVG. 1882 ......47

Cossyphodes .......121

Cossyphus

Cuv. et VaL .....121

Cossyphus

MCCLD. .......6.61

Cottapistus ..............

Cottinella ...........162

Cottiusculus .......161

Cottocomephorus ...162

Cottogaster ........999

Cottogobius ........147

Cottoperca ..........126

Cottopsis ..........161

Cottunculoides ......161

Cottunculus .........161

Cottus ...........161

Cotylephorus ......6.63

Cotylichthys .......153

Cotylis MLr. .......153

Cotylis GTHR. ........153

Cotylopus ........147
Couchia ..........85

Couesius ..........47

Cranoglanis ........59

Crapatalus GILL .....134

Crapatalus GTHR. ...134

Crassilabrus ........122

Crassinarke .........17

Crassispinus .......90

Craterocephalus .....78

Cratinus ...........91

Crayracion ..........34

Creagrudite .........38

Creagrutops ...........38

Creagrutus .........38

Creatochanes ..........38

Creedia ..............125

Creisson ...........147

Cremnobates ........129

Cremnotekla .......129

Crenalticus ..........128

Crenicara ..........116

Crenichthys ........79

Crenicichla ........116

Crenidens .........113

Crenilabrus ........121

Crenimugil .........77

Crenuchus ...........38

Creocele ...........153

Creolus ...........91

Creotroctes .........140

Crepidogaster .......153

Creolus ...........91

Creotroctes ........140

Crepidogaster ........153

Cridorsa ...........113

Crinodus ..........118

Cristatogobius .......147

Cristiceps ..........130

Cristivomer ........24

Crius ............142

Croaltus ...........128

Crockeridius .......130

Croila .............147

Croilia ...........147

Cromeria ..........24

Cromileptes .......993

Cromis ...........108

Crossias ...........161

Crossobothus ........165

Crossocheilichthys ...47

Crossocheilus ......47

Crossochir ..........88

Crossoderma .......157

Crossolepis .........165

Crossolycus ........133

Crossorhinus .......10

Crossorhombus .....165

Crossoscorpaena ......154

Crossostoma .........54

Crotalophis .......70 
Crotalopsis .........70

Cruantus ..........128

Cruriraja ...........15

Cruxentina ........42

Cryodraco .........126

Cryptacanthodes .....131

Cryptacanthoides .....131

Cryptocentroides ...147

Cryptocentrops ......145

Cryptocentrus

(EHBG.) C. et V. . . . 147

Cryptocentrus

(EHBG.) B.

Cryptolynchus

Cryptophehus ........173

Cryptophthalmus
Cryptops

Cryptopsaras .......172

Cryptopterenchelys ...770

Cryptopterus

Gürthr. .........58

Cryptopterus Kaup ...70

Cryptopterygum ...770

Crvptosmilia .........113

Cryptotomus ........123

Cryptotrema ........130

Crystallaria ........99

Crystallias .........164

Crystallichthys .....164

Cristallodytes ........125

Crystallogobius ......148

Ctenichthys ..........130

Citenobrycon ........38

Ctenochaetus .......137

Ctenocharax .........38

Ctenochirichthys ....172

Ctenochromis ......116

Ctenocorissa .......121

Ctenodax ..........143

Ctenodon

NILSSN. .........131

Ctenodon

Swns.

Ctenodon

BPTE.

Ctenodon

'Swns.) Klzgr. . . ...137

Ctenoglyphidodon ....120

Ctenoglyphiorton .........

Ctenogobiops ........148

Ctenogobius ........148

Ctenolabrus .........121

Ctenolates .........93

Ctenolucius ........41

Ctenophallus ........76

Ctenopharyngodon ...447

Ctenopoma .........143

Ctenopristis ........15

Ctenops ..........143

Ctenosciaena .......108

Ctenoscolopsis .....107
Ctenoscopelus

Ctenotrypauchen

Cualac ...........79

Cubanichthys ........79

Cubiceps ...........143

Cugupuguacu ........93

Culaea ...........75

Culius .............145

Culter ............47

Culticula .........47

Cultrichthys .......47

Cultriculus ........47

Cultrops ..........47

Cumbel ............159

Cunningtonia ........116

Curimata .........42

Curimatella ........42

Curimatichthys .....42

Curimatoides .......38

Curimatopsis .......42

Curimatorbis .......42

Curimatus .........42

Curioptera .........167

Currupiscis .........156

Curtipenis ........81

Curtus ...........144

Cyanichthys ..........35

Cyathochromis ......116

Cyathopharynx .....116

Cybiosarda .......139

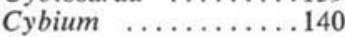

C'ycleptus ........44

Cyclichthys ........35

Cyclocheilichthys ....47

Cyclogaster GRONOW .164

Cyclogaster $(G$.)

BRK. ............ 164

Cyclogobius .........148

Cyclolumpus .......163

Cyclonarce .........17

Cyclophichthys ......70

Cyclopium .......64

Cyclopsetta .........165

Cyclopsis .........163

Cyclopterichthys ....163

Cyclopterocottus .....163

Cyclopteroides ......163

Cyclopteropsis .......163

Cyclopterus ........163

Cyclothone ........26

Cyema ..........72

Cygnodraco .........126

Cylindrosteus ........19

Cymatoceps .........111

Cymatogaster GrBBS ..119

Cymbacephalus .....159

Cymolutes ........122

Cynaedus Gronow ...111

Cynaedus (G.) Scop. . . 111

Cynaedus Swns. .....121
Cyneichthys ........128

Cynias ................

Cynichthys .......93

Cynicoglossus ......167

Cynocephalus KLEIN ..11

Cynocephalus (K.)

GILL ...........11

Cynocharax .........38

Cynodon SpIX .......38

Cynodon Campos ....38

Cynodonichthys .....81

Cynoglossa .........167

Cynoglossoides

v. BND. . . . . . . . 169

Cynoglossoides

Sмітн .........169

Cynoglossus .......169

Cynolebias ........79

Cynomacrurus ......84

Cynopanchax ......79

Cynoperca .........100

Cynophidium ......134

Cynopoecilus .......79

Cynoponticus .....6.68

Cynopotamus ........38

Cynopsetta ..........167

Cynosaartes ........128

Cynoscion ..........101

Cynothrissa ........22

Cynotilapia ..........116

Cypho ..............96

Cyphocharax .......38

Cyphomycter .......137

Cyphoscopelus .......31

Cyphosus ..........113

Cyphotilapia .......116

Cyprichthys .........34

Cyprinella .......47

Cyprinion ........47

Cyprinocirrhites ....118

Cyprinodon ........79

Cyprinogobius .......148

Cyprinopsis BPTE. ...47

Cyprinopsis BLCHD. ..47

Cyprinus ...........47

Cypselichthys ......142

Cypselurus ........75

Cypsilurus .........75

Cyrene ..........47

Cyrtocara ..........116

Cyrtocharax ........38

Cyrtorhynchus ........31

Cyrtus ............144

Cytharinus ........43

Cyttoides ..........90

Cyttoidops .........90

Cyttomimus ........90

Cyttopsis .........90

Cyttosoma .........90 
Cyttula Cyttus

90
90

\section{D}

Daba

Dacentrus .........119

Dacodraco ...........126

Dactylagnus ..........134

Dactylanthias .......91

Dactyleleotris .........145

Dactyleptus .........132

Dactylobatus ..........15

Dactylopagrus .......119

Dactylophallus ........80

Dactylophora ........118

Dactyloptena .........164

Dactylopterus .......164

Dactylopus ..........127

Dactylosargus .......118

Dactyloscopus .......134

Dactylosparus .......119

Dactylostomias ......28

Dacymha ..........107

Dadynos ............133

Daector ...........152

Daemomanta .......17

Daia ..............154

Daicocus ..........164

Dajaus ............77

Dalatias ............13

Dallia ...............30

Dalophis .............70

Damalichthys ......119

Damania ..........131

Dameus ............66

Dampieria .........96

Dampierosa .........157

Danaphos .........26

Danaphryne ........172

Dangilia ..........47

Danglia ...........47

Danichthys ........75

Danio ...........47

Danioides ..........47

Daniops ..........47

Dannevigia ..........135

Darienichthys .......81

Daruma ............160

Dascillus ........22-120

Dascyllus ............120

Dasson .............128

Dastilbe ...........22

Dasyacanthurus ......137

Dasyatis ............16

Dasybatis ........... 15

Dasybatus KLEIN .....16

Dasybatus BPTE. . .....15

Dasybatus (K.) GRMN. .16
Dasybatus (K.-W.)

OgILby ...........16

Dasycottus .........161

Dasyscopelus ..........31

Datnia Cuv. .......111

Datnia Cuv. et VAL. .108

Datniodes .........108

Datnioides ..........105

Daurada ............111

Davalla .......57-62

Davidia ..........139

Davidijordania .....133

Daya ............120

Deania ..............13

Deaniops ...........13

Decadactylus ........44

Decagrammus ......158

Decapogon

Egmn, et Egmn. . . . . 65

Decapogon RIBR. ....103

Decapterus BLKR. ....103

Decapterus POEY ....103

Decodon ...........121

Decterias ...........154

Dekaya .............101

Deleastes ..........157

Dellichthys ........153

Delolepis ..........131

Delothyris .........165

Delsmania .........29

Deltatylosurus ......74

Deltentosteus ........ 148

Deltistes .........44

Delturus ...........65

Demicoilia .........24

Demiurga ............14

Demoisellea ........120

Denarusia .........95

Dendrochirus ....154-169

Dendroscorpaena .....154

Denius ............111

Dentex ............111

Denticeps ..........24

Dentiraja ...........15

Deportator ........58

Derepodichthys ......134

Derichthys ........66

Derjugina .........133

Dermatias .........172

Dermatocheir .......38

Dermatogenys ......74

Dermatolepis ......93

Dermatopsis .......134

Dermatopsoides ......134

Dermatorus ........134

Dermatostethus .....882

Dermocassis .......59

Dermogenys .......74

Dermosteira ........127

Deschauensella......143
Desmoamia .......998

Desmoholacanthus ...114

Desmoprenes . . . . . . 114

Deuteracanthus ......91

Deuterodon ...........38

Deuterophysa .......54

Deuteropterus ......999

Devario .........47

Deveximentum ......106

Devisina ..........96

Dexillichthys .......169

Dexillus ...........169

Dexiourius .........169

Dexistes ............ 167

Diabasis ............107

Diabolichthys .......17

Diabolidium ........173

Diacantha ........54

Diacope ...........105

Diademichthys .....153

Diagramma ........107

Diagramella .........107

Dialarchus .........161

Dialommus ........130

Diana ............90

Diancistrus ........134

Dianema ..........57

Diapaletoplites .....6.65

Diaphanichthys ....669

Diaphasia .........136

Diaphoroculius ......145

Diaphus ............31

Diapoma ...........38

Diapterus .........109

Diastatomycter ......58

Diastobranchus .....68

Diastodon .........121

Dibranchichthys ....171

Dibranchopsis ......171

Dibranchus ..........171

Dicallionymus ......127

Dicentrarchus ......95

Diceratias .........173

Dicerobatus .........17

Dicerophallus ......79

Dichistius .........113

Dichotomycter .......34

Dichotomycterus .....34

Dichrotomycter ......34

Dicologlossy. ......... 168

Dicologoglossa .....168

Dicotylichthys .......35

Dicrolene ..........135

Dicromita ..........135

Dicrossus ..........116

Dicrotus ...........141

Dictynopterus .......88

Dictyosoma ........132

Didymophysa .......54

Dietrichthys ........68 
Dikellorhynchus ....101 Dilepidion ..........148

Dillonia ...........47

Dilobomycter ........34

Dimalacocentrus .....122

Dinectus ...........18

Dinematichthys .....135

Dinematochirus ....28

Dinemus .........88

Dinichthys .........156

Dinogunnellus .......131

Dinolestes .........98

Dinoperca ..........93

Dinotopteroides .....61

Dinotopterus .......6. 61

Diodon .............35

Diodyxodon ........112

Diogenichthys .......31

Dionda ...........47

Dioplites ..........97

Diphreutes .........114

Diphyacantha $\ldots \ldots .81$

Diplacanthopoma ....135

Diplanchias .........36

Diplecogaster .......153

Diplectrum .........91

Diplesion ...........99

Diplobatis ..........17

Diplocheilichthys ....47

Diplocheilus .......47

Diplocrepis ........153

Diplodus ..........111

Diplogrammus .....141

Diplogonurus ......141

Diplolepis ...........108

Diplolychnus .......27

Diplomystax .......56

Diplomyste DUMRL. ...56

Diplomystes (D.)

BLKR. ..........56

Diplomystus ........24

Diplophos ........27

Diplophysa ........54

Diplophysoides ......54

Diploprion ..........104

Diplopterus ........144

Diplospinus ........141

Diplotaxodon .......116

Diproctacanthus ....122

Dipterodon LACPD. ....998

Dipterodon Cuv. ....113

Dipterygonotus ......112

Dipturus ..........15

Diptychus .........47

Dipulus ..........135

Diretmus JHNS. . . . . 88

Diretmus GTHR. .....141

Dirrhizodon .........11

Disceus .............16

Discobatis .........16
Discobatus ..........15

Discognathichthys ....47

Discognathus ......47

Discogobio ........47

Discolabeo .........47

Discolophius ........170

Discopyge .........17

Discotrygon ........16

Discus ............88

Disparichthys .......136

Dissomma .........32

Dissostichus .......126

Distichodina ........43

Distichodomicrura ...443

Distichodura .......43

Distichodus

ML. et Trschl. . . . 43

Distichodus GILL . . . 120

Distoechodon .......47

Distoechus ...........38

Ditrema ..........119

Ditropichthys ......89

Dixiphichthys ......156

Dixiphistes ........156

Dixiphistops .......156

Dixonina .........20

Djabud ............108

Djulongius ........74

Dobula ...........47

Docimodus ........116

Dodecatrema ........7

Doderleinia ........93

Doiichthys .........56

Dolichallabes ......61

Dolichodon .........127

Dolichopteryx .......20

Dolichostomias ......28

Doliichthys ........148

Doliodon ..........103

Dollfusetta ........165

Dollfusichthys ......169

Dollfusina ...........165

Dolloa ............84

Dolloidraco .........126

Dolopichthys ........172

Doraops ...........57

Doras ...........57

Doration ..........99

Doratonotus .......122

Dormitator ........145

Dorosoma .........23

Dorsuarius .........113

Dorybelone ........74

Dorychromis .......120

Doryichthys ........82

Doryptena .........148

Doryrhamphus .....8.82

Dotalabrus ...........122

Doumea .........6. 60

Doydixodon .........113
Draciscus ...........163

Draconetta .........127

Draculo ...........127

Drepane ...........113

Drepanichthys ......113

Drepanopsetta .......167

Drepanoscorpis ......113

Drombus ..........148

Dropsarus .........85

Dubiblennius ........128

Dules ...........95

Dules Cuv. .........91

Dulichthys .......92

Dulosparus ........111

Dunckerocampus ......82

Duohemipteronotus ..122

Duopalatinus ......62

Dupouyichthys .....63

Dussumieria .......23

Duxordia .........59

Duymaeria ..........122

Dysalotus ..........127

Dysichthys .......6.63

Dysomma .......6.68

Dysommina ......6.68

Dysommopsis ......68

- E -
Ebinania $\ldots \ldots \ldots \ldots 161$

Ebisinus ........... 164

Ebisus .............158

Ebomegobius .........148

Ebosia .............154

Echelus ...........70

Echemythes .......87

Echeneis ...........154

Echidna ..........66

Echiichthys ........125

Echinomacrurus .....84

Echinophryne ......171

Echinorhinus .......14

Echinosolea .......168

Echiodon ..........136

Echiopsis ...........70

Echiostoma ........28

Eckloniaichthys ......153

Ecsenius ...........128

Ectenias .......91-142

Ectodus ..........116

Ectrepopterus .......38

Ectreposebastes ......154

Edelia .............95

Edriolychnus .......172

Ehirava .........22

Eichwaldia ........148

Eichwaldiella ........148

Eigenmannia ........44

Eigenmannina .......42

Eillichthys .......47 


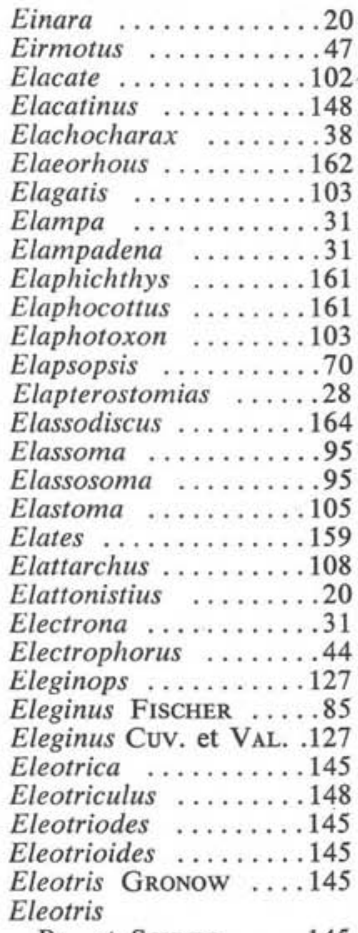

BL. et SCHNDR. ....145

Elephantichthys .......164

Elephenor ...........90

Eleria .............103

Eleutheractis ........93

Eleutherochir ........78

Eleutheronema .......79

Eleuthurus ........666

Elipesurus ...........16

Ellerkeldia .........93

Ellerya .............148

Elliops ............59

Ellischthys ........6.65

Ellochelon ..........77

Ellogobius ..........148

Ellopostoma ........55

Ellops ..............18

Elops LinnÉ ...........19

Elops (C.) LACPD. .....122

Elops (C.) BPTE. ....122

Elopichthys ........47

Elopomorphus ......42

Elxis ..............54

Embassichthys ......165

Embiotoca ...........119

Emblemaria ........130

Emblemariopsis .......130

Embolichthys .......136
Embryx ..........133| Entosphenus .......

Emissola ............11 Entoxychirus .........13

Emmeekia ........122 Enypnias .........148

Emmelanthias ......92 Eomyrophis .......70

Emmelas ..........154 Eomyrus ..........70

Emmelichthyops ....104 Eonemachilus ......54

Emmelichthys .......104 Eopeyeria .........56

Emmnion ........130 Eopsetta ............167

Emmydrichthys .....157 Eosebastes .........154

Empetrichthys .....80 Epalzeorhynchus .....48

Emphycus ........85 Epapterus .........57

Enantioliparis .......164 Epelytes ........92-93

Enantiopus .........116 Eperlanio .........25

Encaeura ..........145 Eperlanus GMRD. ....25

Encheliophiops .....136 Eperlanus BsLWKI. ...25

Encheliophis .......136 Ephippicharax .......38

Encheloclarias ......6. Enhippion ..........34

Enchelybrotula .....135 Ephippus .........113

Enchelycore ........67 Epibulus .......... 122

Enchelycotte ......6.67 Epiceratodus ........173

Enchelynassa ......67 Epicopus ..........85

Enchelyopus Gronow .133 Epicyrtus ..........38

Enchelyopus KLEIN ..141 Epidesmus .........87

Enchelyopus (K.) Epigeichthys .......131 BLKR. ..........141

Enchelyopus

BL. et SCHNDR. . ...85

Enchelyurus ........128

Encrasicholina .......24

Encrasicholus (C.)

LACPD. ..........24

Encrasicholus FLMG. ..24

Encrotes ...........79

Endeconger .........70

Endorrhis ...........58

Enedrias ...........132

Englottogaster .......47

Engraulicypris ......47

Engrauligobius ......148

Engraulis .........24

Engyophrys ........165

Engyprosopon .......165

Enigmapercis ....... 125

Enixe .............113

Enneacanthus ......97

Enneacentrus ......993

Enneanectes .........130

Enneapterygius ......130

Enneistus ..........93

Ennichthys .........119

Enobarbichthys .....54

Enophrys ...........160

Enoplosus ..........112

Entelurus ..........82

Enteromius ........47

Entomacrodops .......128

Entomacrodus ...128-130

Entomocorus .......55

Entomolepis ..........38

Entonanthias ........92
Epigeichthys $\ldots \ldots \ldots .69$
Epigonus $\ldots \ldots \ldots \ldots .69$

Epimonus .........139

Epinephalus ........993

Epinephelides ......93

Epinephelus ........93

Epinnula ...........141

Epipedorhynchus ......34

Epiphthalmus ........145

Epiplatys .........79

Episema .........48

Epitomynis .......24

Epitrachys .........100

Eptatretus .........7

Eques .............108

Equetus ...........108

Equula ............106

Equulites .........106

Eremichthys .......48

Eremophilus ......6.64

Erethistes .........60

Erethistoides .......58

Eretmichthys ........135

Eretmodus .........116

Eretmophorus ......87

Ereunias ...........162

Ericara ...........20

Ericentrus .........130

Erichaeta .........97

Ericius ...........88

Ericosma ..........99

Ericteis ...........130

Ericymba .........48

Eridacnis ...........11

Erilepis ...........158

Erimonax ........48

Erimystax $\ldots \ldots \ldots 48$ 


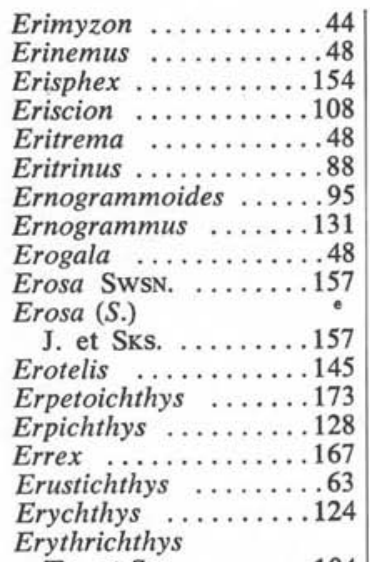

TK. et SgL. ........104

Erythrichthys BPTE. ...38

Erythrichthys MEEK ...116

Erythrinus (P.) LACPD. .88

Erythrinus GRONOW ...38

Erythrinus (G.) Scop. . . 38

Erythrinus Agsz. . . . . 38

Erythrobussothen ....998

Erythrocles ..........104

Erythroculter ........48

Erythrodon ........138

Escadodus .........128

Escolar ..........141

Escualosa .........22

Eslopsarum ........78

Esloscopus ..........134

Esomus . . ........48

Esosynodus ..........30

Esox ................30

Estevea ..........48

Estrella ..........100

Esunculus ........20

Eteira .............114

Etelides ...........105

Etelinus ...........105

Etelis ............105

Eteliscus ..........93

Etheostoma RAFNSQ. .100

Etheostoma

JD. et Evmn. . . . . . 100

Ethestoma ..........100

Ethmalosa .........22

Ethmidium ........22

Etmopterus ..........13

Etroplus ...........116

Etropus ...........165

Euanemus .........57

Eubalichthys .......139

Eubleekeria .........106

Eucalia ..........75

Eucentrarchus .......97
Eucentronotus .....134

Euchalarodus .........167

Euchilichthys ......62

Euchiloglanis ......6.60

Euchilomycterus ......35

Euchoristopus .......152

Eucinostomus ......109

Eucirrichthys ......48

Eucitharus .........165

Euclichthys .......86

Euclyptosternum ....60

Eucrossorhinus ......10

Eucrotus ..........142

Euctenogobius .......148

Eucyclogobius ......148

Eucynopotamus .....38

Eudontomyzon .......7

Eudulus ..........92

Eudynama .........111

Euelatichthys .......107

Eugaleus ...........11

Eugerres ...........106

Eugnathichthys .....43

Eugnathogobius ....148

Eugnathosaurus .......32

Eugomphodus ........10

Euhypsocara .......121

Eulamia ...........11

Euleptorhamphus ....74

Euleptocephalus .....69

Euleptorhampus .....74

Eulophias ........132

Eumakaira .........140

Eumecichthys ......87

Eumeda ..........58

Eumegistus ........90

Eumesogrammus ....131

Eumicrotremus ......163

Eumycterias ........34

Eunarce ............17

Eupemis ............122

Eupetrichthys ......122

Euplatygaster ......22

Eupleurogrammus ....141

Eupnoea ...........169

Eupomacentrus .....120

Eupomotis ........97

Euporista .........170

Euproserpa .......26

Euprotomicrus ......14

Euristhmus ........58

Europus ...........165

Eurumetopos ....107-142

Euryarges .........78

Eurycaulus .......74

Euryglossa .........169

Eurymen .........162

Eurymyctera ......667

Eurypegasus .......165

Eurypharynx
Eurypleura ........169

Eurystola ..........78

Eurystomus

Eusalpa ..........113

Euscarus ..........124

Euschistodus .......120

Eusebastes ..........154

Eusolea ............168

Eusphyra ..........12

Eustira .........48

Eustomatodus ......103

Eustomias FHL. .....28

Eustomias VLNT. . ...28

Eutaeniichthys ......148

Eutaeniophorus .....33

Eutelichthys ........164

Eutherapon .........108

Euthynnus .........140

Euthyopteroma .....106

Eutrigla ..........156

Eutropiellus

Eutropiichthys ......61

Eutropius ........6.61

Eutrumeus ........23

Eutychelithus ........108

Eutyx ............133

Euxiphipops ........114

Evapristis ........107

Evarra ..........48

Evemichthys ........38

Evenchelys .......67

Evepigymnus .......103

Evermanella

FOWLER .........32

Evermannella

EIGENMANN .......38

Evermannia .......148

Evermanniana .....161

Evermannichthys ...148

Evermannolus ......38

Eviota ............145

Eviotops ..........145

Evistias ..........115

Evistiopterus ........115

Evistius ..........142

Evolantia ..........75

Evoplites ...........105

Evorthodus ........148

Evoxymetopon .....141

Evynnis ...........111

Exallias ...........128

Execestides ........126

Exerpes ...........130

Exilichthys ........58

Eximia ............161

Exocallus ...........113

Exocoetus ........75

Exodon ...........38

Exoglossops .......48

Exoglossum ........48 


\begin{tabular}{|c|c|c|}
\hline Exoles & Fodiator & Galeus VALMT. .. \\
\hline Exolissus $\ldots \ldots \ldots \ldots 156$ & Fodifoa ... & Galeus KLEIN .. \\
\hline Exomegas $\ldots \ldots \ldots \ldots 8$ & Foetorepus ........ & Galeus RAFNSQ. . \\
\hline Exonautes .........75 & Fonnio ... & Gallichthys .... \\
\hline Exostoma ..........60 & Fontinus ......... & Gallus ... \\
\hline Expedio & Forbesella .......... & Gambusia \\
\hline Exotirichthys ..... & Forcipiger ....... & Gambusinus \\
\hline Extrarius $\ldots \ldots \ldots \ldots 48$ & Formosania ....... & Ganoideus .. \\
\hline Exyrias ...........148 & Forskalichthys .......69 & Gardonus ........ \\
\hline & rygion $\ldots . .$. & eus ........... \\
\hline F & $\begin{array}{l}\text { Fowlerella } \ldots \ldots \ldots \ldots 122 \\
\text { Fowleria } \ldots \ldots \ldots \ldots \ldots 98\end{array}$ & $\begin{array}{l}\text { Gargariscus } \ldots \ldots \ldots \\
\text { Gargilius } \ldots \ldots \ldots\end{array}$ \\
\hline acciolella $\quad . . .$. & erichthys ..... & Garichthys ......... \\
\hline Fagasa ............145 & Fowlerina ...........38 & ella ...... \\
\hline Fagea ............. 148 & Franciscodoras .... & Garmanichthys ... \\
\hline ula $\ldots \ldots \ldots$. & Franzia & Garmanina...... \\
\hline Falcularius $\ldots \ldots \ldots 48$ & ella....... & Garmannia ....... \\
\hline Fareleotris ..........145 & Fucomimus ........ & $\ldots \ldots \ldots$ \\
\hline Faremusca $\quad \ldots \ldots \ldots 89$ & ....... & \\
\hline Farer $\ldots \ldots \ldots \ldots \ldots 89$ & chthys $\ldots . .$. & canthus ......75 \\
\hline .............106 & opanchax ... & elecus \\
\hline Farhians ........ & losoma ...... & \\
\hline$\ldots \ldots \ldots \ldots \ldots \ldots 24$ & Fundulus $\ldots \ldots \ldots \ldots 79$ & Gas \\
\hline nella $\ldots \ldots \ldots \ldots 26$ & $\ldots \ldots \ldots \ldots$ & \\
\hline iscis ....... & $\ldots$. & sma \\
\hline ella $\ldots . . . .$. & $l a \ldots \ldots \ldots$. & $e a \ldots . .$. \\
\hline igobius .........148 & ianus $\ldots . . .$. & teus ... \\
\hline 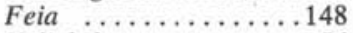 & $\mathrm{Fu}$ & tokeus. \\
\hline thys $\ldots \ldots \ldots$. & nis $\ldots \ldots \ldots$ & $\ldots .$. \\
\hline ia $\ldots \ldots \ldots \ldots$ & $u s \ldots \ldots \ldots$ & anchus \\
\hline tris . & $i x \quad \ldots \ldots \ldots$ & athus. \\
\hline lex $\ldots$. & 48 & $m b a \ldots$ \\
\hline Cuv. ........ & ius $\ldots . . .$. & yzon $\ldots .$. \\
\hline Risso . . . . & ricaria $\ldots$. & mus \\
\hline$\ldots \ldots \ldots \ldots$ & n....... & hysus .... \\
\hline fer $\ldots \ldots \ldots \ldots$ & tgia . & ietta ... \\
\hline$\ldots \ldots \ldots \ldots$ & & terus .... \\
\hline sa $\ldots \ldots \ldots \ldots$. & G & cyphus .... \\
\hline pus $\ldots . . .$. & & tomus \\
\hline ius ............79 & 13 & $\ldots \ldots \ldots \ldots$ \\
\hline$r a \ldots .$. & 154 & 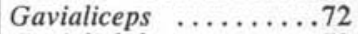 \\
\hline upea ..... & .86 & ys ... \\
\hline ares ....... & ulus $\ldots . . . .$. & $\operatorname{charax} \ldots$ \\
\hline torpedo ... & 84 & $\ldots$. \\
\hline n......... & is RCHDSN. . & \\
\hline$\ldots \ldots \ldots \ldots \ldots 156$ & psis FIPPI. . .86-135 & lus \\
\hline ia $\ldots . . . \ldots$ & $\ldots \ldots$ & tes \\
\hline .81 & 60 & $n$ \\
\hline gobius .......148 & psarus ... & G \\
\hline ria $\ldots . .$. & opsarus . . & thus... \\
\hline omias & cus & thus \\
\hline$s \ldots \ldots \ldots \ldots \ldots 13$ & .26 & deus..... \\
\hline .89 & & adius \\
\hline 167 & thys & us \\
\hline Floridichthys $\quad \ldots \ldots \ldots 79$ & scerdo..... & emus ... \\
\hline ........ & & $\operatorname{arax} \ldots$ \\
\hline & & rus \\
\hline ola $\ldots \ldots \ldots \ldots 148$ & lamnoides .. & Genyonemus .. \\
\hline Flu & hinus & \\
\hline & & \\
\hline
\end{tabular}

ANN. PARASITO. HUM. ET COMP., 1962, 37, $\mathrm{n}^{\circ} \mathbf{6}^{\text {bis }}$ (fasc. suppl.) 


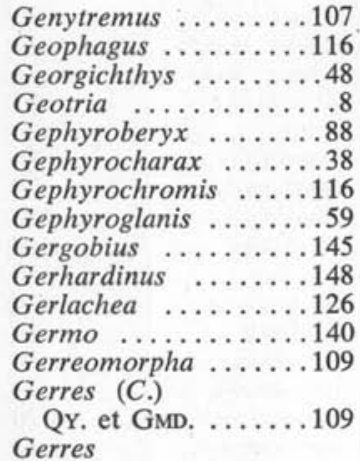

JD. et Evmn. . . . . 109

Ghanan ............107

Gibberichthys .......89

Gibbonsia .........130

Gibelion ..........48

Giffordella ..........128

Gigantactis ........172

Giganthias ........92

Gigantogobius .......148

Gigantura ..........87

Gigliolia ..........73

Gignimentum ......145

Gila .............48

Gilbertella WAITE ....93

Gilbertella EgmN. .....38

Gilbertia ..........92

Gilbertidia ..........162

Gilbertina .........162

Gilbertolus ..........38

Gilchristella ........23

Gilchristia ........115

Gillellus ..........134

Gillia ............ 148

Gillias ................ 130

Gillichthys .........148

Gillisqualus .........11

Gilloblennius ........128

Ginglymostoma .....10

Girardinichthys ......80

Girardinus .........81

Girella ..............113

Girellichthys .......113

Girellipiscis .......113

Girellops ..........113

Giscenchelys .......71

Giton ............ 44

Giuris ............145

Glabrilutjanus .......105

Glabrobarbus........48

Gladiogobius ........148

Gladiunculus ........75

Glanapteryx ........64

Glanertichthys ......115

Glandulocauda ........38
Glanidium .........57

Glaniopsis .........55

Glanis A. 1829 ......56

Glanis A. 1856 ..... 58

Glanis Gronow .....20

Glaphyrodus ........10

Glaridichthys ......81

Glaridodon ........81

Glaridoglanis ......6.60

Glaucosoma ........105

Glaucostegus ........14

Glaucus KLEIN ......103

Glaucus (K.) BLKR. . . 103

Glaucus (K.) FwLR. . . 103

Glaucus (K.) JD. HBs. .103

Gloriella ............128

Glossamia ........98

Glossonodon .......26

Glossichthys .......170

Glossodon RAFNSQ. . ...20

Glossodon HKL. .....20

Glossodus .........20

Glossogobius .......148

Glossolepis ........76

Glossoplites .........97

Glyphidodon ........120

Glyphidodontops ...120

Glyphisodon .......120

Glyphodes .........113

Glyptauchen ....154-157

Glyptocephalus .....167

Glyptoparus .......128

Glyptophidium .....135

Glyptosternon ......60

Glyptosternum ......66

Glyptothorax .....660

Gnathacanthonotus ...73

Gnathacanthus .....158

Gnathagnoides ......126

Gnathagnus .........126

Gnathanodon .......103

Gnathobagrus ......59

Gnathobolus .......22

Gnathocentrum ......136

Gnathocharax .......38

Gnathodentex ........106

Gnathodolus .......42

Gnathodon ............103

Gnathogobius ........148

Gnatholepis .......148

Gnathonemus ......29

Gnathophis .......69

Gnathopogon ......48

Gnathostomias .....28

Gnathypops .........124

Gobatinus .........148

Gobatus .............148

Gobica ..........148

Gobicula ..........148

Gobiculina ..........148
Gobidus ..........148

Gobiella ...........148

Gobiesox ..........153

Gobiex ............148

Gobiichthys ......148

Gobilepres .........152

Gobileptes ..........148

Gobio KLEIN ....... 148

Gobio Cuv. ........48

Gobiobarbus .......48

Gobiobotia .......54

Gobiochromis ......116

Gobiocichla .......116

Gobioclinus ........130

Gobiodon .........148

Gobiodonella .........148

Gobiohelpis .......148

Gobioides ........152

Gobiolepis ..........148

Gobiomoroides ......145

Gobiomorphus ......145

Gobiomorus .......145

Gobionellus ..........148

Gobionichthys .....48

Gobiopsis ........148

Gobiopterus .......148

Gobiopus .........148

Gobiosoma GRD. . . . 148

Gobiosoma Dвwк. ....48

Gobiotrichonotus ....125

Gobius ............148

Gobitis ..............54

Gobulus ..........148

Goeldiella ........62

Gogrius ...........59

Golem ...........171

Gomphosus .........122

Gonenion ..........102

Gonialosa ........23

Goniichthys ..........31

Goniistius .........118

Goniobatis .........16

Goniodermus ........33

Goniodus .............14

Gonionarce .........17

Gonioperca ........92

Gonioplectrus .......93

Gonocephalus .......164

Gonochaetodon ......114

Gonoproktopterus ....48

Gonoproktopterys ...48

Gonopterus ........136

Gonorhynchus

McCld. ........48

Gonorhynchus

Gronow ........29

Gonorhynchus SCOP. . 30

Gonostoma RAFNSQ. ...26

Gonostoma v. HsLT. ..23

Gonostomyxus ......77 


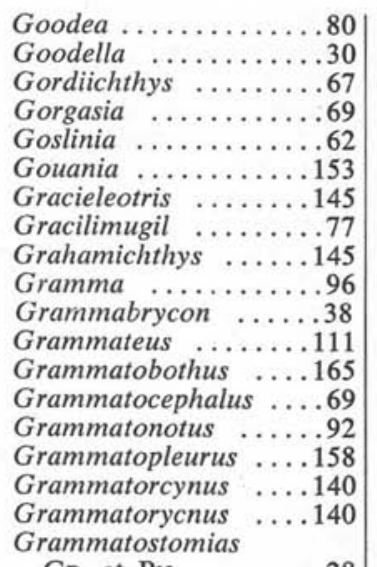
GD. et BN. .......28 Grammatostomias HT. et BYE. .......28

Grammatotria .......116 Grammichthys ..168-170 Grammicolepis ......90 Grammiconotus .....74 Grammistes .........993 Grammistops ......993 Grammonoides .......135 Grammoplites ......159 Grandisquamachela ...48 Graodus ..........48 Graus ............121

Graviceps ..........128

Greeleya ..........161

Gregoryina ........113

Gremilla ...........100

Grenurus ..........84

Griffinetta ........115 Grimaldia .........25 Grimaldichthys ......135 Grimatroctes ......20 Gristes ...........997 Gronias ..........59 Gronovichthys ......98 Grundulus ..........38 Grystes ...........97 Guaperva ..........103 Guavina ..........145 Gudusia ..........22 Guentheridia .........34 Guichenotia .......113 Guiritinga ........56 Gulaphallus ........76 Gulapinnus .........81 Gularus ...........19 Gulliveria ..........998 Gunnamatta .......148 Guntheria ........122 Gunnellichthys .....132
Gunnellops ........132 Gunnellus .........132 Gurnardus .........156 Guttigadus .........86 Gymnacanthus .....161 Gymnachirus .......168 Gymnallabes .......61 Gymnapistes .......154 Gymnapogon .......98 Gymnarchus ......29 Gymneleotris .......145 Gymnelichthys .....133 Gymnelis ......133-136 Gymnelopsis .......133 Gymnepignathus .....103 Gymnetrus .........87 Gymnobatrachus ...152 Gymnobutis .......145 Gymnocaesio ........107 Gymnocanthus .....161 Gymnocephalus BLOCH ..........100 Gymnocephalus Cocco ...........142 Gymnochanda

FSR. BRNR. . . . . . 995 Gymnochanda BSMN. ..95 Gymnocharacinus ....38 Gymnocirrhites ......118 Gymnoclinus .......132 Gymnocorymbus .....38 Gymnocranius .....106 Gymnocrotaphus ...114 Gymnocypris ......48 Gymnodiptychus ....48 Gymnogaster

Gronow .......141 Gymnogaster BRNCH. ..87 Gymnogeophagus ...116 Gymnognathus .....48 Gymnogobius ......149 Gymnolycodes ......164 Gymnomuraena ....667 Gymnopropoma GILL . 121 Gymnopropoma

F. et B. ........121 Gymnopsis .......67 Gymnorhamphichthys .43 Gymnosarda .......140 Gymnoscopelus ......31 Gymnosimenchelys ...66 Gymnostomus ......48 Gymnotes ........44 Gymnothorax BLocH ..67 Gymnothorax GNTHR. .67 Gymnotichthys ......38 Gymnotorpedo .......17 Gymnotus L. $1758 \ldots 44$ Gymnotus L. 1766 ...44 Gymnura ...........16
Gymnurus ........152

Gynutoclinus .......130

Gyrinichthys .......164

Gyrinocheilops ......55

Gyrinocheilus ......55

Gyrinochylus ......48

Gyrinomimus .......89

Gyrinurus ........64

Gyrionemus .......141

Gyropleurodus .......9

$\mathrm{H}$

Habrolepis .........48

Hadropareia ........133

Hadropterus ........100

Haemomaster .......64

Haemonaster ........64

Haemulon ..........107

Haemulopsis .......107

Haemylum .......... 107

Hainania .........48

Halaelurus ..........12

Halaphya ........26

Halargyreus .........86

Halatractus .......103

Haletta ............124

Halex .............106

Halias ............135

Halicampoides .....82

Halicampus ........82

Halichoeres ........122

Halicmetus .........171

Halidesmus ..........132

Halieutaea .........171

Halieutella ..........171

Halieutichthys .....171

Halieutopsis .......171

Haligenes ...........116

Haliichthys ........82

Halimochirurgus .....138

Halimochirus ......138

Halimuraena ........132

Halinanodes .......122

Haliophis ......67-132

Haliperca ..........92

Halisauriceps .......20

Hallecula ..........22

Halmablennius ......128

Halobatrachus .......152

Halocypselus ........75

Halophryne ........152

Haloporphyrus .....87

Halosaurichthys .....73

Halosauropsis ......73

Halosaurus .......73

Halsydrus ..........99

Hamatichthys ......42

Hamiltonia ........997

Hampala ..........48 
Hanno .............149

Hannoichthys ......149

Hanomanctus ........139

Hapalogenys .......107

Haploblepharus .....12

Haplochilichthys .....79

Haplochilus ........79

Haplochiton ........26

Haplochromis ......116

Haploclonus .......28

Haplocylix ..........153

Haplodactylus .......118

Haplodon .........114

Haploglossa ........7

Haploidonotus ......108

Haplophryne .......173

Haplostomias ......28

Haplotaxodon .......116

Haplozebrias ........168

Hara ............60 60

Harengula ........22

Harengus GeOFY. . ...22

Harengus CATESBY ...22

Harengus KLEIN .....22

Harpage ..........89

Harpagifer ...........126

Harpe ............121

Harpochirus ........113

Harpodon ...........30

Harpurina ........137

Harpurus . . . . . ...137

Hatha .............156

Harriotta . . . . . . . 18

Harttia ...........65

Hasemania ........38

Hassar ............65

Hatcheria .........664

Hatumeus .........154

Haustor ...........59

Hazeus ............149

Hectoria .........993

Hedinichthys .......54

Heegerius . .........48

Hegerius ..........48

Helcogramma ......130

Helgia ...........55

Heliases ...........120

Heliastes ...........120

Helicobranchus ......29

Helicolenus ........154

Helicophagus .......66

Heioperca .........97

Helmichthys ......669

Helmictis .........71

Helminthodes ........136

Helminthostoma ....136

Helops BRWN. ...993-121

Helops

BRDT. et RATZBg.
Helostoma . ........112

Helotes .......... 108

Helotosoma .......144

Hemerocoetes .......125

Hemerorhinus .......71

Hemiancistrus ......6.65

Hemianthias ........92

Hemiarius ........56

Hemibagrus ........59

Hemibalistes ........138

Hemibarboides .....48

Hemibarbus .........48

Hemibates .........116

Hemibrycon ..........38

Hemicaranx .......103

Hemicetopsis .......64

Hemichaetodon ......114

Hemichromis .......116

Hemiconiatus ........34

Hemicoris ........122

Hemiculter .......448

Hiemiculterella ......48

Hemicurimata ......42

Hemicyclodon ......127

Hemidoras .........55

Hemieleotris .......145

Hemiemblemaria ....130

Hemiexocoetus ......75

Hemigaleops ........11

Hemigaleus .........11

Hemiglyphidodon ...120

Hemigobius .........149

Hemigrammalestes ....38

Hemgirammocapoeta

PELgRn. ........48

Hemigrammocapoeta

Estev. .........48

Hemigrammocharax ...38

Hemigrammocypris . ..48

Hemigrammonannocha-

$\operatorname{rax} \ldots \ldots \ldots \ldots . . .38$

Hemigrammopetersius .38

Hemigrammopuntius ..48

Hemigrammus '.......38

Hemigymnus ........122

Hemijulis ..........122

Hemilepidotus .....160

Hemiloricaria ......6.65

Hemilutjanus .......99

Hemimacrurus .......84

Hemimarsupium .....82

Hemimyzon ........55

Heminodus .........157

Hemiodon .........65

Hemiodontichthys ....665

Hemiodopsis ......43

Hemiodus . . . . . . . ...44 43

Hemicplites .........997

Hemipimelodus ......56

Hemiplatystoma .....62
Hemiplus ..........448

Hemipsilichthys ....665

Hemipterois .........32

Hemipteronotus ....122

Hemirhamphodon ...75

Hemirhamphus ......75

Hemirhombus .......165

Hemisalanx ........25

Hemiscapirhynchus ....18

Hemisciaena ........108

Hemiscyllium .......12

Hemisilurus .........58

Hemisorubim .....6.62

Hemistichodus .......43

Hemistoma ........124

Hemisynodontis ......62

Hemitaurichthys ....114

Hemitautoga .......122

Hemithylacus .......82

Hemitilapia .........116

Hemitremia ........48

Hemitriakis .........11

Hemitripterus ......162

Hemitrygon .........16

Hemiulis .........122

Hemixiphophorus ....81

Henicichthys .......118

Henicorhynchus .....48

Heniochus ..........114

Henochilus ........38

Henonemus ........64

Hepatoscartes ........128

Hepatus GRoNow . . . 137

Hepatus (G.)

JRD. et SL. . . . . . 137

Hepatus Röse .......99

Hephaestus ........108

Hephthocara ........135

Hepsetia .........78

Hepsetus ...........38

Heptadecacanthus ....120

Heptanchus .........9

Heptapterus ........62

Heptatrema ........7

Heptatretus ........7

Heptranchias ........99

Herichthys ........116

Heringia .........22

Herklotsella ......22-58

Herklotsichthys ......22

Herklotsina .........136

Hermosilla ........120

Herops ............95

Heros .............116

Herpetoichthys (S.)

GNTHR. .........173

Herpetoichthys

KaUP ..........71

Herrea WhTLY. .......149

Herrea Sмітн .......149 


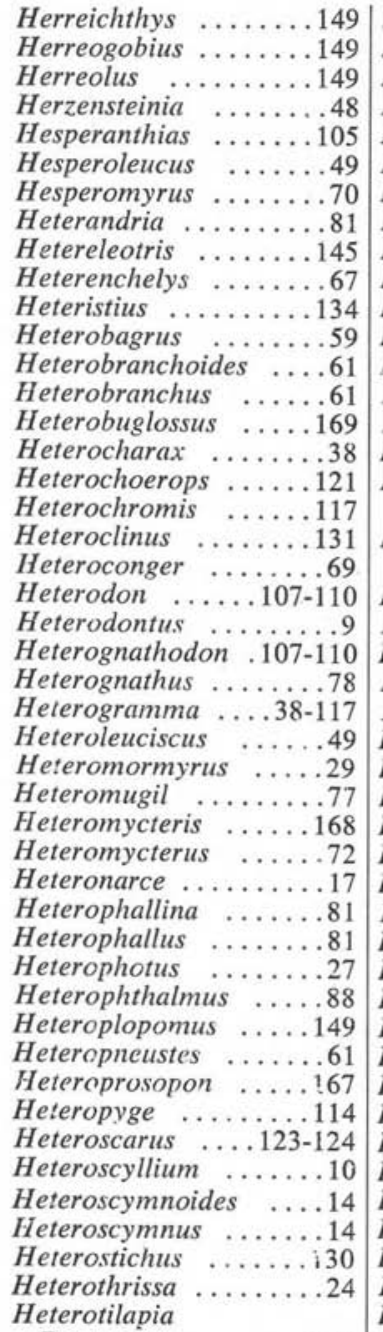

PeLLGRN.

Heterotilapia

REGAN

Heterotis ............117

Heterythrinus .........38

Hexabranchus ........7

Hexacanthus ........149

Hexagrammoides ....158

Hexagrammos ........158

Hexagrammus .......158

Hexanchus ..........9

Hexanematichthys .....56

Hiatula .......121-122

Hierichthys ........132
Hierops ............31

Hieroptera ...........15

Higanfugu .........34

Hildebrandella ......103

Hildebrandia .......69

Hildebrandichthys ....22

Hilgendorfia .........164

Hilsa .............22

Himantolophus .....172

Himantura .........16

Hime ..............30

Hinalea ..........122

Hingobio .........49

Hintonia ...........31

Hiodon ..........20

Hippichthys ........82

Hippocampus

RAFSe.

82

Hippocampus

LeACH .........82

Hippocampus Cuv. ....82

Hippocephalus ....163

Hippoglossina .......166

Hippoglossoides ....167

Hippoglossus .......167

Hippohystrix ........82

Hipposcarus .......124

Hipposcorpaena ......154

Hippopotamyrus .....29

Hippurus .........91

Hirculops ........128

Hirundinichthys .....75

Hirundo ...........75

Hisonotus .........65

Hispanicus ........154

Histiobranchus ....6.68

Histioclinus ........130

Histiocottus ........160

Histiodromus ......42

Histiogamphelus .....83

Histiophorus .......140

Histiopterus .......115

Histrio ...........171

Histriobranchus ......68

Histriodraco ........127

Histriophryne ......171

Hito Haigg. . . . . . ...58

Hito Herre . ........58

Hitoichthys ........58

Hobar ..............105

Hogbinia ...........110

Holacantha ........114

Holacanthus LACPD. . . 114

Holacanthus GRONOW .34

Holanthias .........92

Holcomycteronus .....135

Holconotus ........119

Holesthes ...........39

Hollandichthys ......39

Hollardia .........138
Holobrycon .........39

Holocentrum .......89

Holocentrus GRoNow . .89

Holocentrus (G.)

Scop ...........89

Holodus . . ..........9

Hologenes ........6.62

Hologymnosus .....122

Holohalaelurus ......12

Hololepis ...........100

Holonodus .........169

Holoprion ..........39

Holopristis ..........39

Holopterura ........70

Holorhinus .........16

Holoscorpaena .....154

Holoshesthes .......39

Holotaxis ...........39

Holotrachys .......89

Holoxenus .........158

Holtbyrnia ........21

Homalogrystes ..994-120

Homalopomus ......85

Homaloptera ........55

Homalopteroides ......55

Homalopterula ......55

Homatula ...........54

Homea .............7

Homesthes ........128

Homodemus .......94

Homodiaetus .......64

Homodon .........105

Homolenus ........83

Homoprion . . . . . . . 108

Homostolus .......135

Hopladelus ........59

Hoplarchus .......117

Hoplerythrinus ......39

Hoplias ...........39

Hoplichthys ........159

Hoplisoma .........65

Hoplobrotula .......135

Hoplochromis ......120

Hoplocoryphis ..........442

Hoplocottus ........161

Hoplodoras .........57

Hoplognathus ......110

Hoplolatilus .........101

Hoplomyzon ......6.63

Hoplonotus ........156

Hoplopagrus ........105

Hoplophycis . . ......136

Hoplopteryx ........88

Hoplosebastes .......154

Hoplosternum ........ 5

Hoplotethus .......88

Hoplotilapia .......117

Hoplunnis .........69

Horandandia .......49 49

Horaglanis .......6. 61 


\begin{tabular}{|c|c|c|}
\hline & Hyphalonedrus & 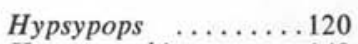 \\
\hline Horalabiosa .......49 & Hyphessobrycon ... & Hyrcanogobius \\
\hline Hospilabrus ........ & Hypleurochilus ... & Hyrtlinus \\
\hline Howella ......... & Hyplophus ...... & Hysterocarpus \\
\hline Hozukius & Hypnarce ......... & Hysteronotus .... \\
\hline ubbesia & Hypnos ........ & Hystricodon $\ldots .$. \\
\hline Hubbsichthys .... & Hypoatherina & \\
\hline Hubbsiella .......78 & Hypocaranx ......103 & I \\
\hline .........79 & Hypoclydonia ......999 & \\
\hline Hucho & Hypoclinemus ......168 & \\
\hline$\ldots \ldots \ldots$ & Hypocolpterus .......65 & Iburiella \\
\hline ichthys $\ldots \ldots \ldots$ & Hypocryptichthys ...119 & Iburina \\
\hline Humejordia ........106 & Hypodis ..........103 & Icania \\
\hline$\ldots \ldots \ldots 97$ & Hypodoras ..........57 & Icelichthys ..... \\
\hline$\ldots \ldots \ldots$ & ytes $\ldots \ldots \ldots 154$ & Icelinus ... \\
\hline cceratias $\ldots . .$. & $H y$ & Icelus \\
\hline yalorhynchus ..26-159 & logobius & Ichthaelurus \\
\hline nathus ......49 & us $\ldots \ldots \ldots 100$ & lis . . \\
\hline$\ldots \ldots \ldots$ & Hypolophus ....... & Ichthyacus ........ \\
\hline nchus .......49 & $\ldots \ldots 153$ & Ichthyapus .....667-71 \\
\hline ira $\ldots \ldots \ldots$ & rus .....154 & borus ........43 \\
\hline gyra .........79 & icus $\ldots \ldots \ldots 42$ & Ichthycallus $\ldots . .$. \\
\hline ........39 & $\ldots 25$ & obus ..........44 \\
\hline nus $\quad . . \ldots .$. & michthys ..49 & campus .. \\
\hline$\ldots \ldots \ldots$ & hthalmus $\quad . \ldots 62$ & Ichthyococcus .... \\
\hline onichthys .. & odes ... & colla ...... \\
\hline Hydrocyonoides .... & Hyp & ocoris ... \\
\hline lagus $\ldots \ldots \ldots \ldots 18$ & chilus ....128 & oelephas ... \\
\hline cus $\ldots . . . \ldots$ & 135 & yzon ... \\
\hline$\ldots \ldots$ & .......105 & ......6.67 \\
\hline rdus ....... & us $\ldots \ldots \ldots 43$ & ogon ....... \\
\hline lox ........ & Hy & mphos \\
\hline onus $\quad \ldots \ldots \ldots 117$ & don & oscopus .. \\
\hline zon $\ldots \ldots \ldots \ldots 44$ & $s \ldots \ldots$ & hys $\ldots \ldots \ldots \ldots 142$ \\
\hline gnathus ... & $m a \ldots 65$ & 143 \\
\hline halus ... & 136 & .59 \\
\hline gadus ... & hus ... & 143 \\
\hline us .... & .94 & .44 \\
\hline hysa .... & Hy & gon \\
\hline physa .... & $s \ldots \ldots \ldots \ldots 65$ & nthus $\ldots \ldots \ldots \ldots 27$ \\
\hline ..... & 163 & piscis \\
\hline lus & & \\
\hline$\ldots \ldots$ & rus .......59 & hthys ... \\
\hline urus ... & us $\ldots$. & ectes. \\
\hline us $\ldots \ldots$. & hus & hthys. \\
\hline$m a \ldots \ldots$. & etes .... & a $\ldots \ldots$ \\
\hline mus . & & Illa \\
\hline & & Ilic \\
\hline tus $\ldots . .$. & .49 & Ilictis \\
\hline & & Ilisha \\
\hline$\ldots \ldots \ldots 110$ & 120 & 149 \\
\hline ium $\ldots \ldots \ldots 44$ & $\ldots \ldots \ldots 85$ & Illana \\
\hline oristus. & $.86-87$ & Iinocoetes \\
\hline us ........97 & niops . . . . 128 & on .. \\
\hline & nius & .68 \\
\hline yphe ...107-142 & & \\
\hline pisus $\quad \ldots \ldots$ & $\operatorname{chax} .$. & Imhoffichthys \\
\hline$\ldots$ & Hyp & 100 \\
\hline & Hyp & rales \\
\hline Hyperprosopon .. & Hypsurus & Imparfinis \\
\hline
\end{tabular}




\begin{tabular}{|c|c|}
\hline Incisidens $\quad . \ldots \ldots \ldots 114$ & Isuropsis \\
\hline Incisilabeo ..........49 & Isurus. \\
\hline Indialosa ........ & Itaiara $\ldots$ \\
\hline Indocybium .......140 & Itatius ............135 \\
\hline Indomanta $\ldots \ldots \ldots \ldots 17$ & Itbaya...$\ldots \ldots$ \\
\hline Indosphyraena .... & Iulis ...... \\
\hline Indostomus $\quad \ldots \ldots \ldots 76$ & \\
\hline Inegocia ..........159 & $-\mathbf{J}-$ \\
\hline Inermia ........104-112 & Jadamga \\
\hline Infratridens $\ldots . \ldots 153$ & Jamsus. \\
\hline Iniistius $\quad . \ldots \ldots \ldots \ldots 122$ & Japanopsychrolutes \\
\hline Inimicus $\ldots \ldots \ldots \ldots 157$ & Japonoconger .......69 \\
\hline Innoculus $\ldots \ldots \ldots$ & Jenkinsia . . \\
\hline 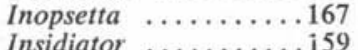 & Jenkinsiella ........71 \\
\hline $\begin{array}{l}\text { Insidiator } \ldots \ldots \ldots \ldots 159 \\
\text { Insopiscis }\end{array}$ & Jenynsella ........ \\
\hline $\begin{array}{l}\text { Insopiscis } \ldots \ldots \ldots \ldots \ldots 159 \\
\text { Intonsagobius } \ldots \ldots \ldots 149\end{array}$ & Jenynsia . . \\
\hline 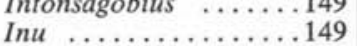 & Jerdonia ..........120 \\
\hline Iou $\ldots \ldots \ldots \ldots \ldots \ldots \ldots 100$ & Jobertina $\quad \ldots \ldots \ldots \ldots 43$ \\
\hline Ioamia.......... & Johnius $\ldots \ldots \ldots \ldots$ \\
\hline Ioglossus $\ldots \ldots \ldots \ldots 145$ & $\begin{array}{l}\text { Johnsonina } \ldots \ldots \ldots \\
\text { Joinvillea }\end{array}$ \\
\hline Iosillago ...........101 & $\begin{array}{l}\text { Joinvillea } \\
\text { Jordanella }\end{array}$ \\
\hline Iotichthys .........49 & $\begin{array}{l}\text { Joraane } \\
\text { Jordania ................ }\end{array}$ \\
\hline Iotogobius $\ldots \ldots \ldots 149$ & Jordanichthys ........105 \\
\hline Ipnoceps $\ldots \ldots \ldots \ldots 32$ & Jordanicus ....... \\
\hline Ipnops ............ & Jordanidia ... \\
\hline ........ & Jordanites $\ldots . . .$. \\
\hline cypris $\ldots \ldots \ldots \ldots$ & Joturus ..............77 \\
\hline Iredaleichthys $\ldots \ldots 120$ & Julichthys \\
\hline Iredalella...$\ldots \ldots$ & Julidio . . \\
\hline Irex $\ldots \ldots \ldots \ldots \ldots$ & Julidochromis \\
\hline $\begin{array}{l}\text { Iridio } \ldots \ldots \ldots \ldots \ldots \\
\text { Irillion }\end{array}$ & Julis Cuv. . . \\
\hline $\begin{array}{l}\text { Irillion } \ldots \ldots \cdots \cdots \cdots \\
\text { Irolita }\end{array}$ & Julis GNTHR. \\
\hline $\begin{array}{l}\text { Irolita } \\
\text { Irvineia }\end{array} \ldots \ldots \ldots \ldots \ldots \ldots{ }^{15}$ & Juncurus . . \\
\hline Isacia $\ldots \ldots \ldots \ldots \ldots \ldots$ & Juvenella ... \\
\hline Isaciella $\ldots \ldots \ldots \ldots 107$ & \\
\hline ops $\ldots . . \ldots \ldots$. & \\
\hline Ischikaunia ...... & Kajikia \\
\hline chnomembras ... & Kali \\
\hline nosoma ....... & Kamoharaia \\
\hline Isesthes ...... & Kanazawaichthys \\
\hline Isichthys $\ldots \ldots \ldots$ & Kanekonia . \\
\hline Isistius $\ldots \ldots \ldots \ldots$ & Kanduka . . \\
\hline 78 & Kantaka \\
\hline Isobuna & Kantapus \\
\hline phalus..... & Kareius .... \\
\hline atha ........ & Kasatkia ... \\
\hline Isogomphodon ... & Kathetostomus . \\
\hline Isonotus & wonus ..... \\
\hline Isopisthus ....... & Kaupichthys . \\
\hline Isoplagiodon $\quad \ldots \ldots \ldots 11$ & Kaupus ... \\
\hline etta $\ldots \ldots \ldots$ & Kelloggella \\
\hline lennius ... & Kendallia . \\
\hline Istigobius . . . . . . & $\bar{K}$ enoza ........ \\
\hline Istiompax .... & Kentrocapros ... \\
\hline omax & Keris \\
\hline Istiophorus ..... & Kessleria \\
\hline hombus. & Ketengus \\
\hline & \\
\hline
\end{tabular}

Ketengus

BLKR. $1858 \ldots \ldots .56$

Kirtlandia .........78

Kishinoella ........ 140

Klamatella ........49

Kleiwegia .........159

Klunzingerina ......96

Kneria ..........21

Knipowitschia ......149

Knodus ............39

Koinga ...........13

Konopickia ........95

Konosirus .........23

Korsogaster ........88

Kosswigichthys .....79

Koumansetta .......149

Kowala .............22

Kraemeria .....125-152

Kreffitus ......... 145

Kribia ......... 145

Krohnutus .........84

Kronia ..........78

Kronichthys ......65

Kroseriphus .......108

Krusensterniella .....133

Kryptopterichthys ...58

Kryptophanaron .....88

Kryptopterus .....58

Kuhlia .........95

Kumococius ......159

Kurandapogon .....98

Kurtus ............ 144

Kurtaflammeo .......89

Kyleia ............. 165

Kyphosus .......113

$$
\text { - L - }
$$

Labeo Cuv. ........49

Labeo BowDCH. .......111

Labeobarbus RupL. . . . .49

Labeobarbus BLKR. . . 49

Labeotropheus .....117

Labeova ...........111

Labichthys .........72

Labidesthes ........78

Labidochromis .....117

Labidorhamphus ....75

Labracinus ........96

Labracoglossa ...... 142

Labracopsis .........94

Labrastrum ........123

Labrax KLeIN .......95

Labrax (K.) Cuv. ....95

Labrax PaLlas ......158

Labrichthys ........ 123

Labrisoma ........130

Labrisomus .........130

Labristoma . . . . . . . .996

Labrochromis REGAN . . 117 


\begin{tabular}{|c|c|c|}
\hline & Lampichthys & otherapon \\
\hline Labroclinus & Lamprichthys & Leiopsis BNNTT. \\
\hline Labroides & Lampris ........... & Leiopsis BPTE. \\
\hline Labroperca & Lamprogrammus .. & Leiostomus \\
\hline Labropsis ........ & Lamprologus .... & Leiosynodontis \\
\hline Labrosomus ...... & Lamprossa $\quad . . .$. & Leirus ........ \\
\hline Labrus...$\ldots \ldots$ & Lamprotoxus ..... & Leisomus .... \\
\hline Laccoeleotris ..... & Lampugus ....... & Leitectus .. \\
\hline pedia $\ldots \ldots \ldots .92-118$ & Lanceabarbus ..... & Leluranus \\
\hline Lachneria .........98 & Landonia ..........39 & Leiurus KAUP. \\
\hline Lachnolaimus ......121 & Lanioperca ........98 & Leiurus $(K$.$) DumL.$ \\
\hline noloemus ......121 & Lappanella .........121 & Leiurus SwSN. ..... \\
\hline arius ........... & Laputa ... & Leius ....... \\
\hline ophrys ........33 & Larimichthys ..... & Lembesseia . . \\
\hline .33 & Larimodon & Lembus .. \\
\hline tricola ....... & Larimus .........109 & Leme. \\
\hline tris $\ldots \ldots \ldots$. & Larvicampus ........83 & nisoma .. \\
\hline avia $\ldots \ldots \ldots \ldots$ & mcistrus $\ldots \ldots \ldots 65$ & $L$ \\
\hline zargus ....... & Lasiognathus .......172 & Leoblennius \\
\hline nolyta $\ldots \ldots \ldots$ & Latargus $\quad \ldots \ldots \ldots \ldots 129$ & Leocottus .. \\
\hline$m a \ldots \ldots \ldots 86$ & Latebrus $\ldots . . \ldots \ldots$ & hthys \\
\hline lodes & Lateolabrax ........ & Lepadogaster ....... 153 \\
\hline modus ... & Lates ............95 & Lepibema ..... \\
\hline$\ldots \ldots \ldots$ & Lathargus .......... & $h a$ \\
\hline chthys $\ldots \ldots \ldots$ & Latilus & alochromis \\
\hline chthys $\ldots \ldots \ldots 137$ & Latimeria & ia $\ldots . . . \ldots$. \\
\hline ja $\ldots \ldots \ldots$ & dopsis & lois . \\
\hline 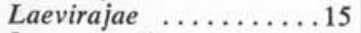 & Latr & Le \\
\hline ratias ....... & Latroblennius ... & blennius \\
\hline halus $\quad . .$. & $\ldots \ldots$ & \\
\hline ila $\ldots \ldots \ldots$ & ulodes ... & ennius \\
\hline$\ldots \ldots 111$ & ulus .... & \\
\hline lla $\ldots$. & $c a$. & $L$ \\
\hline phalus ..... & Laurida ... & alichthys \\
\hline ......... & $i a \ldots . .$. & is $\ldots .$. \\
\hline$\ldots \ldots$ & 149 & don \\
\hline .61 & Leb & romis ... \\
\hline$m a \ldots$ & 79 & ium $\ldots . .$. \\
\hline & 39 & $L$ \\
\hline aathus .... & tes Sмitт . . . . . 149 & bius ........149 \\
\hline$\ldots \ldots$ & tes FLPI. ... & prologus \\
\hline & 58 & rus \\
\hline 166 & iogaster & $\ldots .54$ \\
\hline$s \ldots \ldots$ & yia $\ldots$. & gas $\ldots . .$. \\
\hline dra .... & & $L$ \\
\hline & .62 & hanes ... \\
\hline ichthys ... & $L$ & mus \\
\hline & atus KLEIN & 107 \\
\hline 11 & atus RFNSQ. . & etta GILL \\
\hline & tus BLVIL. .. & etta GNTHR. . \\
\hline & sis & \\
\hline 108 & tus $\ldots . .$. . & ygopsis \\
\hline$m a$ & $n$ SwNS. . & idus ... \\
\hline 140 & OO OWEN ... & inus \\
\hline ichthys ... & don WooD. .. & ombus \\
\hline & eotris. & Lepidorhynchus \\
\hline todes & & .140 \\
\hline iyctus & lossus. & Lepidosiren \\
\hline & athus & Lepidosoma \\
\hline & & \\
\hline
\end{tabular}


Lepidothynnus

Lepidotrigla ..........156

Lepidotus .........90

Lepidozygus .......120

Lepimphis .........91

Lepinannocharax ......43

Lepiopomus .........97

Lepipinna ........42

Lepipterus ..........109

Lepisacanthus ......88

Lepisoma ..........130

Lepisosteus .........19

Lepodus ...........90

Lepogenys .........20

Lepomis ...........97

Lepophidium ........136

Leporellus ........42

Leporinodus .......42

Leporınus ........42

Leprogaster ........139

Leptacanthichthys ....172

Leptagoniates ........39

Leptagonus ..........163

Leptanthias ........92

Leptarius ..........56

Leptaspis .........103

Leptecheneis ........154

Leptenchelys .......70

Lepthaemulon ........107

Leptoancistrus .......65

Leptobarbus (B.) 1859

Leptobarbus $(\boldsymbol{B}$.) 1863

Leptoblennius

Leptobotiu .........5

Laptobrama ........98

Leptobrycon .........39

Leptocarcharias ......11

Leptocarias .........11

Leptocephalichthys ...69

Leptocephalus

GRONOW

Leptocephalus

SCOPOL

Leptocephalus

BASLWSK. ........49

Leptocerdale ........133

Leptocharias .........11

Leptochilichthys .....20

Leptochromis BLKR. ...96

Leptochromis REGAN .117

Leptoclinus .........131

Leptocottus .........161

Leptocypris ........49

Leptoderma .........20

Leptodes ...........28

Leptodoras ........57

Leptofierasfer ......136

Leptogadus .........86
Leptogaster .........23

Leptoglanis BLGR. ...59

Leptoglanis EIGMN. . ...62

Leptognathus .......7.71

Leptogobius .........149

Leptogunnellus ......131

Leptoichthys ........83

Leptojulis ..........123

Leptokyphosus ......113

Leptolaeops .........166

Leptolamprologus ...117

Leptolebias ........80

Leptolepis ..........142

Leptoligoplites .......103

Leptolucania .......80

Leptometopon .......114

Leptonotus .........83

Leptonurus .........24

Leptopegasus ......165

Leptoperca .........100

Leptophidium ......136

Leptophilypnus .....145

Leptophycis . .......86

Leptops ...........59

Leptopterygius .....153

Leptopus ..........87

Leptorhamdia ......62

Leptorhaphis ......81

Leptorhinophis ......71

Leptorhynchus SMITH .71

Leptorhynchus LowE 72

Leptoscarus ........123

Leptoscolopsis ........107

Leptoscopus .....125-134

Leptosoma ..........168

Leptostomias ........28

Leptosynanceia .......157

Leptotilapia .........117

Lepturacanthus ......141

Lepturichthys .......55

Lepturus

GRONOW .......84

Lepturus RAsQ. . .....142

Lepturus (ARTD.)

GILL ............141

Lermichthys ........80

Lestidiops ............31

Lestidium .............31

Lestradea ..........117

Lestrolepis ............31

Lesueuria ..........149

Lesueuriella .........125

Leusueurigobius ....149

Lesueurina ...........125

Letharchus .........71

Lethenteron .........7

Lethops ............149

Lethostole .........78

Lethotremus ........163

Lethrinella ..........110
Lethrinichthys ......110

Lethrinops ........117

Lethrinus ..........110

Leucalburnus

BGR. 1914 ......49

Leucalburnus

BgR. 1916 ......449

Leucaspis ..........49

Leucaspius .........49

Leucichthys ........25

Leucicorus ..........135

Leucidius ..........49

Leucisculus .........49

Leuciscus KLEIN .....49

Leuciscus Cuv. ......49

Leucobrotula ........135

Leucochlamys .......135

Leucoglossa .........103

Leucogobio .........49

Leucops ...........100

Leucopsarion ........149

Leucoraja ...........15

Leucos .............49

Leucosoma .......25-49

Leucosomus .......49

Leuresthes .........78

Leuroglossus .......26

Leurynnis ........133

Levanaora .........159

Leviprora ...........159

Lewinichthys .......74

Lhotskia ..........74

Liachirus ..........168

Liauchenoglanis .....59

Lichia ............103

Lichnochromis ......117

Lienardella ........121

Lile ..............22

Limamuraena ......67

Limanda ...........167

Limandella ........167

Limia (P.) 1854 .....81

Limia (P.) $1861 \ldots . .81$

Limiculina .........74

Limnichthys ........125

Limnochromis .....117

Limnocottus .......162

Limnothrissa .......22

Limnotilapia ........117

Limnurgus ..........80

Limparhomaloptera ...55

Lindemanella ........145

Linnocottus ........162

Linophora .........114

Linophryne ........173

Liobagrus ..........59

Liobranchia .........153

Liocaesio ...........107

Liocassis . ..........59

Liocornus ............139 
Liocranium .......155

Lioglossina .........166

Liomonacanthus ....139

Lioniscus ...........18

Lionurus ..........84

Liopempheris ......98

Lioperca ...........94

Liopropoma .......94

Liopsetta .........167

Liopteryx ..........166

Liosaccus ...........34

Lioscorpius ........155

Liostomus .........109

Lioteres ...........145

Lipactis ..........172

Liparis Scop. . . . . . 164

Liparis RösE .......164

Lipariscus ..........164

Liparius ...........164

Liparoides ..........164

Liparops ............164

Liparus ...........49

Lipochromis .......117

Lipogenys ........73

Lipomyzon ........44

Lipophrys ......120-128

Lipopterichthys .....665

Liposarcus .........65

Lirus ...........143

Lissocampus ........83

Lissocheilichthys .....49

Lissochilus .........49

Lissorhynchus .......49

Litanchus ...........128

Lithodoras .........57

Lithogenes .........65

Lithognathus ........111

Litholepis ...........19

Lithoxus .........65

Lithulcus ...........143

Litocara ...........100

Lixagasa .........26

Liza ...............77

Lizagobius .........149

Lizettea ...........146

Lloydiella ..........20

Lo ................ 137

Loa ...............114

Lobianchia ..........31

Lobocheilus ..........49

Lobochilotes ........117

Lobodeuterodon ......39

Lobodus ............111

Lobopterus .........88

Lobotes ...........105

Loligorhamphus ....75

Lomanetia .........76

Lonchistium ........124

Lonchiurus ..........109

Lonchogenys
Lonchopisthus

Lonchurus ............109

Longiculter ........499

Longirostrum ........103

Longitrudis .........159

Longmania ..........11

Longurio ..........49

Lophalticus ........128

Lopharis ...........102

Lophenchelys ......67

Lophidius ...........170

Lophiobagrus .......59

Lophiocharodon ......171

Lophiocharon .......171

Lophiodes .........170

Lophiodon ..........35

Lophiogobius .......149

Lophiomus . . . . . . . 170

Lophiopsides ........170

Lophiosilurus .....662

Lophius ...........170

Lophocephalus ......89

Lophodolos ........172

Lophodolus

Lophogobius .......149

Lopholatilus ........101

Lophonectes ........166

Lophopsetta .......166

Lophorhombus ......166

Lophotes .........87

Lophotopsis ........87

Loricaria .........65

Loricariichthys ....665

Loro ..............124

Lota ............86

Lotella ...........86

Lotta ...........86

Louti ...........994

Lovamia .........98

Lovettia ..........26

Lowenia ............31

Loxodon ...........11

Loxolutjanus ........105

Loxopseudochromis ...96

Lubricogobius .......149

Lubulogobius ........149

Lucania ...........80

Lucaya ..............128

Lucayablennius ......128

Lucifer ...........28

Lucifuga ...........135

Lucigadella ........84

Lucigadus .........84

Luciobarbus .......49

Lucioblennius .......131

Luciobrama ........49

Luciobrotula ........135

Luciocephalus .......144

Luciocharax ........41

Luciocyprinus
Luciogobius .......149

Luciolates ...........95

Lucioperca ..........100

Luciopimelodus .....62

Luciosoma .........49

Luciosudis ...........31

Luciotrutta ........25

Lucius Geof. ........30

Lucius KLEIN .......30

Lucius RAFNSQ. .......30

Lucoscombrus ......141

Lucubrapiscis ..........34

Luetkenia ..........39

Lumpenella ........131

Lumpeneopsis ......131

Lumpenus .........131

Lumpus Cuv. .......163

Lumpus OKEN .....163

Lumpus RAFNSQ. ....163

Lunicauda .........105

Lunolabrus ........123

Lupinoblennius ......128

Luposicya .........149

Lutianus .............105

Lutjanus ...........105

Lutodeira ..........21

Luvarus ..............142

Luxilinus .........49

Luxilus ............50

Luxinus ............50

Luzoneleotris .......146

Luzonichthys ....92-107

Lycenchelys .......133

Lycengraulis .......24

Lychnophora .......31

Lychnopoles .......27

Lycias ..............133

Lycichthys ........129

Lycocara ............133

Lycocyprinus ......80

Lycodalepis ........133

Lycodapus .........134

Lycodes ...........133

Lycodontis ........67

Lycodonus ........135

Lycodophis ........133

Lycodopsis ...........133

Lycogenis ..........107

Lycogramma ........133

Lycogrammoides ....133

Lycondes .........8.84

Lyconectes .........131

Lyconema ...........133

Lyconodes .........84

Lyconus .........84

Lycothrissa .......24

Lycozoarces ........133

Lyocetus .........171

Lyoliparis ..........164 
Lyopsetta ........167

Lyosphaera ..........35

Lyragalaxias .......26

Lyrichthys ........156

Lysodermus .......157

Lythrichthys .......155

Lythrulon .........107

Lythrurus ........50

Lythrypnus ........149

\section{M}

Macaria .......... 140

Maccullochella .....992

Maccullochia ........115

Maccullochina ......998

Macdonaldia .......73

Macgregorella .......149

Machaenichthys .......

Machaera .......... 140

Machaerenchelys .....71

Machaerium ........132

Machaerochilus .....50

Machaerope .......141

Machephilus .......13

Macilentichthys .....106

Macleayina ........83

Macolor ...........105

Macquaria ........994

Macrhybopsis ......50

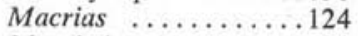

Macristium ........21

Macrocephalus

BRWN.

Macrocephalus (B.)

BLKR.

95

Macrocephenchelys ...68

Macrochirichthys ....50

Macrochoerodon .....121

Macrochyrus .......155

Macrodon ScHz. .....101

Macrodon MLR. .....39

Macrodonophis ......71

Macrodontogobius ...149

Macrognathus

GRONOW '.......74

Macrognathus

LCPD. . . . . . . . . 170

Macrogobius .......149

Macrolepis ........98

Macromastax .......20

Macromystax ........30

Macrones ..........59

Macronichthys .......59

Macronoides ........59

Macronotothen ......127

Macroparalepis ......31

Macropharyngodon ..123

Macropharynx ......36

Macrophthalmia .....8
Macropinna .......25

Macropleurodus ..... 117

Macropodus ........143

Macrops .............105

Macropsobrycon ......39

Macropterobagrus ....59

Macropteronotus .....61

Macropus .........143

Macrorhamphosodes ..138

Macrorhamphosus ...74

Macrorhynchus ..140-141

Macroscorpius ......155

Macrostoma ..........32

Macrostomias .......28

Macrostomoides .....28

Macrotrema .......72

Macrouroides .......84

Macrourus ........84

Macrozoarces ......133

Macrura ..........22

Macrurocyttus ......90

Macruronus ........84

Macruroplus ....... 84

Macrurrhynchus .... 128

Macrurus .........84

Maculocoetus ......75

Madigania ......... 108

Maena .............112

Maenas KLEIN .....112

Maenas (K.)

BLKR.

Maenichthys .......119

Maenioides ..........110

Magnisudis ..................

Mahidolia ...........149

Maina ............ 110

Makaira ........... 140

Malacanthus .........101

Malacobagrus ......62

Malacobatis ........ 15

Malacocanthus .....992

Malacocentrus .....123

Malacocephalus ....884

Malacocottus ........161

Malacoctenus ......130

Malacopterus ........121

Malacorhina ........15

Malacosarcus .......899

Malacosteus ..........32

Malakichthys ......94

Malania ........... 174

Malapterurus ......62

Malapterus ......... 121

Mallotus .........25

Malpuletta ......... 143

Malthae ...........171

Malthe ..........171

Malthopsis .........171

Malvoliophis ......71

Manacopus .......76
Mancalias .........172

Mancopsetta ........166

Mandelichthys .....143

Mandibulacra .......50

Manducus .........26

Mangarinus ......... 149

Mannichthys .......50

Manta .....................

Mapo .................... 149

Mapolamia .........11

Marcgravia ........152

Marcgravichthys ....153

Marcusenius ........29

Margariscus ........50

Margaritodon ......124

Margrethı .......27

Markiana .............39

Mar leyella ....... 167

Marlina .................. 140

Marosia .............138

Marosichthys .......138

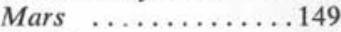

Marsis .......... 112

Marouba ..........83

Marubecula ........ 156

Marnkawichthys .... 164

Mascalongus ........ 30

Massaria ........... 164

Mastacembelus

KLEIN ........74

Mastacembelus

GRONOW .........170

Mastacembelus

SCOPOLI ........ 170

Masticobarbus .......50

Mastigopterus ....133-135

Masturus .....................

Mataeocephalus .....84

Matsubaraea .......125

Matsyu ........... 50

Maugeclupea ......22

Maurolicus ........27

Maxillicosta .......155

Maxillingua .......50

Mayerina ..........6.69

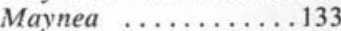

Mayoa ...........50

Meadia ............68 68

Mearnsella .........50

Mebarus ..........155

Mechaenichthys .....120

Meda ............54

Medialuna ..........112

Medicelinus ............... 160

Medipellona ........22

Megaderus .........67 67

Megagobio ........50

Megalamphodus ......39

Megalaspis .............. 103

Megalepis ..........110 
Megalepocephalus ...20 Megalobrama .......50 Megalobrycon .......39 Megalocottus .......161 Megalodoras .......57 Megalolepis .....110-140 Megalonema .......62

Megalopharynx .......36

Megelops ..........19

Megaphalus ........153

Megapharynx .......44

Megaperca ........994

Megaprotodon ......114

Megarasbora ........50

Megastomatobus ......44

Meiacanthus .......128

Mekongina ........50

Meladerma ........102

Melambaphes ........114

Melamphaes .......89

Melanichthys .......138

Melanichthys

TEM. et SCHGL. . . . . 114

Melaniris .........78

Melanobranchus .....84

Melanocetus .......171

Melanochromis ......117

Melanodactylus .....59

Melanogenes . . . . . . 117

Melanogloea .........33

Melanogrammus .....86

Melanonectes ......28

Melinonosoma ......86

Melanonus .........86

Melanorhinus ......78

Melanostigma .......133

Melanostoma ......998

Melanostomias ......28

Melanotaenia ......76

Melantha ..........109

Melanura ............30

Melapedalion ........75

Melbanella ..........103

Meletta ...........22

Melichthys .........138

Melletes ........... 160

Melloina ..........39

Memarchus ........44

Membracidichthys ...159

Membras .........78

Mendosoma .......124

Mene ............104

Menephorus ........994

Meneus ...........104

Menidia BRwn. .....24

Menidia BPTE. ......78

Menidiella .........78

Menticirrhus .......109

Mentiperca .......992

Mentodus
Merinthe .........155

Merinthichthys ......70

Merlangius ........85

Merlangus

RFNSQ. ......... 85

Merlangus (C.) Ок. ...86

Merlinus .........86

Merluccius

RAFNSQ. .........85

Merlucius GRoNow ...85

Merlus ...........85

Merogymnus .......124

Merolepis .........112

Merou ...........994

Merulinus .........156

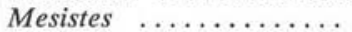

Mesites ...........26

Mesoborus ........43

Mesocottus .........161

Mesocyprinus

CHENG. ..........50

Mesocyprinus

FENG.

Mesogobius .........149

Mesogonistius ......997

Mesomisgurnus ......54

Mesonauta .........117

Mesoprion .........105

Mesopristes .........108

Mesops ...........117

Metacottus .........161

Metagobius ........149

Metahomaloptera .....55

Metallites ..........50

Metopias .........89

Metopomycter ......6.69

Metoponops ........166

Metrogaster .......119

Metynnis .............39

Metzelaaria ........155

Metzia ............50

Meuschenia ........139

Meuschenula .......146

Micracanthus SvgE. ..144

Micracanthus ......114

Micralestes ..........39

Micrapocryptes ......149

Micraspius ........50

Micristius ........80

Micristodus ..........11

Microbarbus ........50

Microbrotula .......135

Microbrycon .........39

Microbuglossus ......168

Microcaelurus .......39

Microcanthus .......114

Microcephalocongrus ..69

Microcharax .......43

Microchirichthys ....28

Microchiropsis ......168
Microchirus RAFNSQ. .168

Microchirus BPTE. ...168

Microconger ......66

Microcottus ........161

Microdesmus .......133

Microdistichodus ....43

Microdonophis ......70

Microdontostomias ...28

Microdus .........42

Microeleotris ........146

Microgadus ........86

Microgaster ........117

Microgenys .........39

Microglanis ......6.62

Micrognathus .......83

Microgobius .......149

Microichthys ......98

Microlepidalestes ....39

Microlepidium .......86

Microlepidogaster ....6.65

Microlepis ........50

Microlophichthys ...172

Micromesistius .....86

Micromesus ........17

Micrometrus .......119

Micromugil ........80

Micronema .........58

Micropanchax ......8 80

Microperca

Castln. ......95-98

Microperca

Putnam ..........100

Micropercops ......149

Microphallus .......81

Microphilypnus .....146

Microphis .........83

Microphysogobio ....50

Micropodus ........123

Micropoecilia ....80-81

Micropogon .......109

Micropogonias .......109

Micropterus .........97

Micropteryx ........103

Micropus KNER .....103

Micropus GRAY ....157

Microrasbora ......50

Microschemobrycon ...39

Microsicydium ......149

Microspathodon ....120

Microstoma ........26

Microstomatichthyobo-

rus ............44

Microstomus .......167

Microsynodontis ....662

Microthrissa .......22

Microtremus .......119

Microtrigla ........156

Milyeringa .........144

Mimagoniates .......39

Mimasea ............141 
Mimocubiceps .......998

Mimoperca .........100

Mindorogobuus ......149

Miniellus ..........50

Minnilus ..........50

Minomus ..........44

Minous ...........157

Minytrema ........44

Mionorus .........998

Miopsaras .........172

Mirapinna ...........33

Mirbelia ............153

Mirognathus ........20

Mirogobius .........149

Mirolabrichthys ...92-105

Mirorictus ........20

Mischominatus .....169

Mischommata ......169

Mischommatus .....169

Misgurnus .........54

Mistichthys .......149

Mitchillina .........20

Mitotichthys .......83

Mitsukurina .........10

Miuroglanis ......664

Mixobrycon .........39

Mixonus ...........135

Mnierpes ..........130

Moapa ...........50

Mobula ..........17

Mochokus .......662

Modigliania ........54

Moebia ...........135

Moenkhausia ........39

Mogurnda .........146

Mohanga ..........80

Moharra ...........110

Mola KLRTHR. .......36

Mola LINCK .........36

Mola Cuv. ..........36

Mola HeCKel .......50

Molacanthus .........36

Molarii ...........667

Mollienesia .........81

Mollienisia .........81

Molochophrys ........9

Molua ............86

Molva .............86

Molvella .........85

Monacanthus .......139

Monacoa ..........25

Monhoplichthys .....159

Moniana ..........50

Monishia .........149

Monistiancistrus ....665

Monocentris ........88

Monoceratias .......172

Monoceros (P.)

LACPD.
Monoceros

BL. et SCHNDR. . . 137

Monochir ..........168

Monochirus (C.) OK. . 168

Monochirus BPTE. ...168

Monochirus KAUP. ...168

Monocirrhus .......115

Monodactylus ......112

Monodichthys .......168

Monognathus ..........36

Monolene ........166

Monomitopus .........135

Monomitra ........164

Monoprion ........98

Monopterhinus ......9

Monopterus ........72

Monosira ..........98

Monostichodus ......43

Monotaxis ..........110

Monothrix ..........135

Monotocheirodon .....39

Monotreta ..........34

Monotretus .........34

Montalbania .......23

Montereya .........161

Moojenichthys .......39

Moolgarda ..........77

Mora ............87

Moralesia ..........39

Moralesicus .......................

Morara ..........50

Mordacia .........

Morhua ..........86

Moringua .........67

Mormyra ..........124

Mormyrhynchus .....42

Mormyrodes ........29

Mormyrops ........29

Mormyrostoma ......57

Mormyrus LINNÉ ......29

Mormyrus CATSBY. . . 110

Moroco ..........50

Morone ...........95

Moronopsis .......95

Morrhua .........86

Morua ..........86

Morulius ...........50

Morwong ..........119

Moseleya .........84

Motella ...........85

Moxostoma ........44

Mrigala ............50

Mucogobius .........146

Mugil ............77

Mugilogobius .......149

Mugiloides .........124

Mugilomorus ........19

Mugilops .........78

Mugilostoma .......149

Mulgoa ............146
Mulichthys .........143

Mullhypeneus .......110

Mulloides .........110

Mulloidichthys ......110

Mullus ...........110

Munrogobius ......150

Mupus ...........143

Muraena ........66

Muraenesox .....6.68

Muraenichthys ......70

Muraenoblenna LACPD. 7

Muraenoblenna KAUP. 67

Muraenoclinus ......130

Muraenoides .......132

Muraenolepis ......87

Muraenophis Cuv. ...67

Muraenophis KaUP ...71

Muraenophis LACPD. . .67

Muraenopsis ......71

Muraenosaurus ......68

Murasoius .........155

Murrayia .........94

Musteia (C.) Ок. ....85

Mustelichthys ......994

Mustellus ...........11

Mustelus VaLmt. ....11

Mustelus Linck .......11

Mustelus LEACH

Mustelus Cuvier .....11

Mycteroperca .......994

Myctophum .........32

Myersglanis .......60

Myersichthys .......130

Myersina .........150

Myersiscus ........127

Mylacrodon ........107

Myleocollops ........39

Mylesinus ...........39

Myletes Cuv. .......39

Myletes

MLr. et TSCHL. . ...39

Myleus ..............39

Mylio ............111

Myliobatis .........17

Mylocheilus .........50

Mylochromis .......117

Myloleuciscus .......50

Myloleucops .........50

Myloleucus COPE .....50

Myloleucus GNTHR. ...50

Mylopharodon .......50

Mylopharyngodon ....550

Myloplus ............39

Mylorhina .........17

Mylossoma .........39

Myoglanis ........6. 62

Myomyrus .......29

Myoxocephalus ......161

Myrichthys .......71

Myriodon .........94 
Myriolepis .........158

Myriosteon .........15

Myripristis .........89

Myrmillo ..........11

Myroconger ........66

Myrophis ..........70

Myropterura .......70

Myrus KaUP ........70

Myrus Cuv. .......770

Mystacoleucus .......50

Mystaconurus .......84

Mystibranchus ......32

Mystidens ..........11

Mystriophis ........71 Mystus

GRONOW $1763 \ldots 56-59$

Mystus (G.) SCOP. . ..59

Mystus KLEIN .......50

Mystus LACPD. ......24

Mystus GRONOW 1854

Mytilophagus .......119

Myxine ...........7

Myxocephalus ......135

Myxocyprinus ......44

Myxodagnus .......134

Myxodes ...........130

Myxonum .........124

Myxostoma .........44

Myxus ...........77

Myzopsetta ........167

$-\mathrm{N}-$
Nandina $\ldots \ldots \ldots \ldots .50$

Nandopsis GILL . . . . 117

Nandopsis MeIKeN ...115

Nandus ...........115

Nangra ...........60

Nannacara REGAN . . .117

Nannacara RIBEIRo ..117

Nannaethiops ......43

Nannapogon .......98

Nannatherina ......76

Nannobrachium .....32

Nannobrycon .......43

Nannocampichthys ...83

Nannocampus ......83

Nannoceratias .......172

Nannocharax ......43

Nannochromis .....117

Nannoglanis .......63

Nannoperca ........995

Nannopetersius .......39

Nannorhamdia .....6.63

Nannostomus ......43

Nanochromis .......117

Nanognathus .......43

Nanostoma .........100

Nansenia ..........26

Naqua .............105
Narcacion KLEIN

Narcacion $(K$.$) B .....17$

Narcetes ............20

Narcine ........... 17

Narcinops . . . . . . . 17

Narcobatis . . .......17

Narcobatus .........17

Narke ..............17

Narooma ..........26

Naseus ..................

Nasisqualias . . . . . 13

Nasistomias .........28

Naso ................ 137

Nasocassis .........59

Nasolychnus ........32

Nasonus .......... . 137

Nasus ............ 5!

Nauclerus .........103

Naucrates ...........103

Naucratopsis ........103

Naurua ..........92

Nautichthys .........160

Nautiscus ...........160

Nautopaedium ......153

Navarchus .........143

Navodon ...........138

Nazatexico ........50

Neacanthopsis .......54

Nealosa ...........23

Nealotus ...........141

Neamia ...........98

Neanis ...........123

Neanthias .........92

Neatypus .........112

Nebris ............101

Nebrius ................10

Nebrodes ............10

Nectamia ..........999

Nectarges ........ 78

Nectoliparis .........164

Nector ............109

Niedystoma ........56

Neenchelys ........68

Neetroplus .........117

Negambassis ........97

Negaprion ..........11

Negogaleus .........11

Negoscartes .........128

Negostegastes .......120

Negotirus ............30

Nelus .............139

Nelusetta .........139

Nemabathytroctes ....21

Nemacheilus ........54

Nemachilichthys .....54

Nemachilus .........54

Nemadactylus .......119

Nemadoras .........57

Nemalycodes ........133

Nemamyxine ..........
Nemanthias .......92

Nemaperistedion ......157

Nemapontinus .......155

Nemapterois ........155

Nemapteryx .......56

Nemasiluroides ......61

Nematabramis .......50

Nematagnus ........126

Nematalosa ........23

Nemateleotris ......146

Nematistius .........104

Nematobalistes ......138

Nematobrotula .....135

Nematobrycon ......39

Nematocentris ...... .76

Nematochromis ......96

Nematodactylus ....119

Nematogenys ......664

Nematogobius .......150

Nematonurus ......84

Nematonus ........135

Nematoparodon ......43

Nematopoma ........39

Nematoprora ......72

Nematops ...........166

Nematostoma .......83

Nematostomias ......28

Nematozebrias .....169

Nemichthys ........72

Nemipterus .........106

Nemobrama .........88

Nemocampsis ......97

Nemochirus .....87-87

Nemophis .........132

Nemotherus ........87

Nemuroglanis ......6.63

Neoanthias ........92

Neoarius .........56

Neoatherina .......76

Neobagrus ........59

Neoblennius .......134

Neobola ...........50

Neoborus BLGR. 1898.43

Neoborus BLgR. 1899 ...39

Neobythites ........135

Neocarassius .......50

Neocentropogon .....155

Neoceratias .........173

Neoceratodus .......173

Neochaetodon .......114

Neochanna ........26

Neochela .........50

Neochromis ........117

Neocirrhites .........118

Neoclinus ..........130

Neoconger ........69

Neocyttus .........90

Neoditrema ........119

Neoepinnula ........141

Neoetropus ........167 
Neofundulus ........80

Neogastromyzon .......50

Neogobius ...........150

Neogunellus ..........134

Neohariotta ..........18

Neohemilepidotus ....161

Neoheterandria ......81

Neohispanicus ........155

Neohomaloptera ......55

Neolabrus ..........123

Neolebias .......43-80

Neolethrinus ........111

Neoliparis ..........164

Neomacheilus ........54

Neomaenis ...........105

Neomerinthe ........155

Neomesoprion .......105

Neomordacia .......8

Neomugil .........77

Neomuraena ........67

Neomyripristis ......89

Neomyxine .........7

Neomyxus ..........77

Neonesthes ........27

Neoniphon CASTLN. ...89

Neoniphon DEVIS ....92

Neoodax ...........124

Neoophorus ........80

Neoopisthopterus ....22

Neopagetopsis .......126

Neopangasius ......6.61

Neoparascyllium ......10

Neopataecus ........158

Neopempheris . ......98

Neopercis ..........125

Neopharynx ........117

Neophos ..........27

Neophrynichthys ....162

Neoplatycephalus ....159

Neoplecostomus .....65

Neoplotosus .........58

Neorhombus .......166

Neopoecilia ..........81

Neorohita ..........50

Neosalanx ..........25

Neoscombrops ......999

Neoscopelarchoides ...32

Neoscopelus ........99

Neoscopelus CASTLN. ...32

Neoscopelus JHSN. ....32

Neoscorpis ..........112

Neosebastes .........155

Neosillago ..........101

Neosilurus STNDCHR. ...58

Neosilurus CAstLn. . . . . 58

Neosphyraena .......999

Neostethus ........76

Neosteus ..........22

Neostoma VAILT. ....27

Neostoma FILHOL . ...27

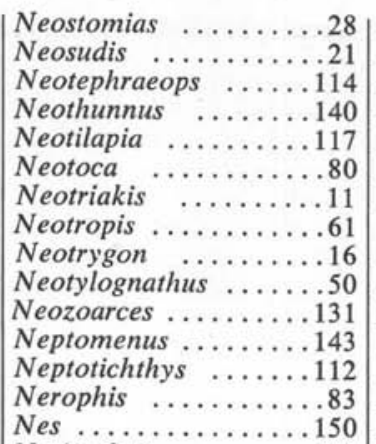

Nesiarchus ..........141

Nesiotes ..........96

Nesocongrus ......6.69

Nesogalaxias ........26

Nesogobius .........150

Nesogrammus ...140-141

Nessorhamphus .....68

Nestis ...........77

Nettastoma .......68

Nettastomella ........68

Nettastomops .......68

Nettodarus ........69

Nettophichthys .....68

Netuma ..........56

Newtonscottia ........30

Nexilaris ............120

Nexilarius ..........120

Nexilosus ..........120

Nezumia .........84

Nibea ................ 109

Nicholsiculter .......50

Nicholsicypris .......50

Nicholsina ..........123

Nicholsopuntius .....50

Nigracus .........83

Nilotica ..........22

Ninnia ............150

Ninnigobius .......150

Niphon ..........994

Nivicola ............100

Niviperca .........100

Nixiblennius . . . . . 128

Nocomis .........50

Nodogymnus ..........168

Nofua ..............157

Nomalus ...........101

Nomarhamphus .....75

Nomeus ...........143

Nominostomias ....28

Nomoctes ..........21

Nomorhamphus ....7.75

Norfolkia .........130

Noriona ...........21

Normanetta ........168

Normania ..........21
Normanichthys .....93

Normichthys .........21

Notacanthus .......73

Notarius ............56

Notastrape .........17

Notemigonus ........50

Notesthes .........155

Nothobranchius .....80

Nothonotus ........100

Notidanus ..........9

Notistium ..........140

Notocheirus .......78

Notoclinops ........130

Notoclinus .........130

Notogaleus .........11

Notoglanidium ......59

Notoglanis . . . .....63

Notognidion ........94

Notogrammus .......131

Notograptus .........134

Notolepidomyzon ....45

Notolepis ...........31

Notolychnus .........32

Notomyxine ........7

Notophthalmus .....6.62

Notopodichthys .....28

Notopogon ........74

Notopterus .......20

Notorabula .......67

Notoraja ............15

Notorhynchus .......9

Notoscopelus .......32

Notosema .........166

Notosudis ...............

Notothenia ........127

Notropis ..........50

Notropocharax .......39

Notrullus ..........170

Noturus ..........59

Novacampus .......83

Novacula CASTBY ...124

Novacula Cuv. ......123

Novaculichthys .....123

Novanthias .......92

Novarchus

Noviricuzenius .....160

Novumbra ...........30

Nuchequula ........106

Nudagobioides ........150

Nudiantennarius .....171

Nudipagellus ........111

Nukta .............50

Nuria ...........50

Nyctimaster ............32

Nyctophus ...........32

Nystactes .........69

Nystactichthys .......69 


\begin{tabular}{|c|c|c|}
\hline - & Ogcocephalus & Ophicephalus \\
\hline & Ogilamia $\ldots \ldots \ldots$ & Ophichthus \\
\hline liquogobius & $\ldots \ldots \ldots$ & Ophichthys \\
\hline tortiophagus & hodes.. & Ophidium \\
\hline 管 & Okamejei ......... & ieleotris \\
\hline 163 & Okkelbergia ....... & oblennius \\
\hline ceanomyzon & Oliglyphisodon ... & Ophiocara \\
\hline Oceanops ... & Oligobrycon .........39 & Ophiocephalops .. \\
\hline obius ... & Oligocephalus ......100 & Ophiocephalus .... \\
\hline nacanthus & ottus ........ & clinops .... \\
\hline tskia $\ldots$ & Oligolepis $\quad . \ldots \ldots$. & linus ...... \\
\hline 9 & Oligoplites.....$\ldots$. & $\ldots$. \\
\hline hthys & Oligopodes ...... & Ophiognathus ... \\
\hline nodon..... & Oligopodus $\ldots \ldots \ldots \ldots 91$ & gobius $\ldots \ldots$ \\
\hline ammus & Oligopus .........91 & hinus $\ldots . .$. \\
\hline ma MARTNS. ...55 & $\ldots \ldots \ldots$ & ion ........ \\
\hline ma Hzstn. ......55 & rcus $\ldots . . . .$. & \\
\hline ematichthys ....59 & orpaena $\ldots .$. & oma \\
\hline nus $\ldots \ldots \ldots \ldots 15$ & rops ........ & nus ......... \\
\hline$\ldots \ldots \ldots \ldots \ldots 19$ & ops $\quad \ldots \ldots \ldots$. & non $\ldots \ldots \ldots 7$ \\
\hline spinus $\quad \ldots \ldots \ldots 133$ & ........ & apus ...... \\
\hline (2) & hthys $\ldots .$. & \\
\hline ... & lon ........ & $a x$ \\
\hline tes & is $\ldots \ldots$. & olepi \\
\hline & hys ..... & olophus \\
\hline 4.) LACPD. & $\ldots \ldots$ & lycus \\
\hline ........ & $r a \ldots$. & pelton \\
\hline$s a \quad \ldots \ldots$ & ichus ... & tilapia \\
\hline nia $\ldots . .$. & ys $\quad \ldots \ldots$ & . \\
\hline$\ldots \ldots$ & $\ldots \ldots$ & dus \\
\hline yopus ... & acentrus .. & \\
\hline ........ & $\ldots \ldots \ldots$ & ntrus \\
\hline$\ldots$. & ......... & athus \\
\hline$\cdots \cdots$ & $\ldots \ldots$ & $a$ \\
\hline tis $\ldots \ldots$. & 96 & ctus \\
\hline inus $\ldots \ldots \ldots$ & 128 & erus \\
\hline$s \ldots$. & halus ... & eilus \\
\hline is & us $\ldots . .$. & \\
\hline hus $\ldots . .$. & es $\ldots \ldots$ & hus \\
\hline ius $\ldots \ldots 150$ & lalus ... & 59 \\
\hline$a x \quad \ldots$ & $\ldots \ldots$ & \\
\hline ... & chus ... & SмITH. \\
\hline crurus ... & $\ldots \ldots$ & ) \\
\hline tes $\ldots .$. & & VAL. \\
\hline & $\cdots$ & pl \\
\hline$\ldots$ & les .... & INR. \\
\hline nphus & $\ldots \ldots \ldots$ & ias ... \\
\hline$\ldots$ & & 153 \\
\hline rnarchus .. & & \\
\hline ... & & hthys \\
\hline hus. & & \\
\hline pstomias ... & .50 & dochromis \\
\hline nops & & $\ldots 111$ \\
\hline is Cocco & athus .. & .........57 \\
\hline & toma $\quad$. & \\
\hline & & dus \\
\hline & & Optiv \\
\hline & genys $\ldots .$. & Optonurus \\
\hline & & \\
\hline
\end{tabular}




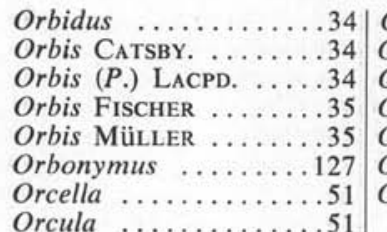

Orcynopsis .........140

Orcynus RAFNSQ. ...103

Orcynus Cuv. .......140

Orectolobus .........10

Oregonichthys .......51

Oreias ...........55

Oreichthys ........51

Oreinus .........51

Oreochromis ........117

Oreoglanis ........60

Oreogobius ........150

Oreoleuciscus .......51

Oreonectes .........51

Oreosoma ..........99

Orestias $\ldots \ldots \ldots \ldots 80$

Orfus ................

Orinocodoras .......57

Orissagobius ........150

Ornichthys ...........156

Oroptychus ........9

Orqueta ..........103

Orthagoriscus ........36

Orthagus .............

Orthichthys .......74

Orthochromis .......117

Orthocolus ........25

Orthocraeros ..........140

Orthodon ..........51

Ortholeucos .........51

Orthomyleus ........40

Orthopnias ........160

Orthopristis ........ 107

Orthopsetta ........166

Orthosternarchus ....44

Orthostoechus ......107

Orthostomus .......150

Orthrias ..........55

Orycnopsis .........140

Orycnus ............ 140

Oryzias ............80

Osbeckia ..........139

Oshimia ............144

Osmeri ............25

Osmerus .........25

Osopsaron ........... 125

Osorina .........68

Osorisa ..........155

Ospatulus ..........51

Osphromenus ......144

Osphronemus ......144

Osphyolax ........83

Osteichthys ......899
Osteobagrus .......59

Osteobrama .........51

Osteochilichthys .....51

Osteochilus ........51

Osteochromis ......115

Osteogaster .......65

Osteogeneiosus

B. $1847 \ldots \ldots \ldots 56$

Osteogeneiosus

B. $1858 \ldots \ldots \ldots 56$

Osteoglossum (V.)

Cuv. ..........29

Osteoglossum BASLWK. .24

Osteomystax $\ldots \ldots \ldots 57$

Ostichthys (LGSDFF.)

CUV. et VAL. . . . . . 89

Ostichthys (LGSDFF.)

JD. et Evmn. . . . . .899

Ostophycephalus .....58

Ostorhinchus ...99-110

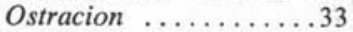

Ostracoberyx $\ldots \ldots .88$

Ostreoblennius .....128

Ostreogobius .......150

Osurus ...........125

Otakia .............. 51

Otalia ...........78

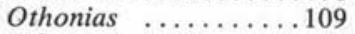

Othonocheirodus ....440

Othonophanes ......40

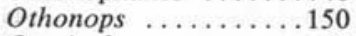

Otocinclus .......65

Otohime .......... 156

Otolithes .............. 101

Otolithoides ..........101

Otolithus ..........101

Otoperca ............ 107

Otopharynx .......117

Otophidium ........136

Otothyris .......665

Otrynter ............111

Ovoides LCPD. .......35

Ovoides Cuv. ........35

Ovoides DuMrL. ......35

Ovum ........................

Owsianka .........51

Owstomia

Owstonia ........... 126

Oxuderces $\ldots \ldots \ldots 125$

Oxybarbus .........51

Oxybeles ...................

Oxycephas .......84

Oxychaetodon .....115

Oxycheilinus .......123

Oxycirrhites ........118

Oxyconger ........69

Oxycottus $\ldots \ldots \ldots 161$

Oxydoras ..........57

Oxyeleotris $\ldots \ldots \ldots 146$

Oxygadus $\quad \ldots \ldots \ldots . . .64$
Oxygaster .......51-55

Oxygeneum ........51

Oxyglanis .......6. 62

Oxyjulis ....................

Oxulabrax ........995

Oxylebius $\ldots \ldots \ldots . . \ldots 158$

Oxyleyna $\ldots \ldots \ldots \ldots 83$

Oxyloricaria .......66

Oxymacrurus ......84

Oxymetopon .......150

Oxymonacanthus ...139

Oxymormyrus ......29

Oxymugil ..........77

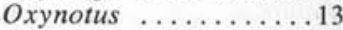

Oxyodon .........97

Oxyporhamphus .....75

Oxyrhina ......... 10

Oxyropsis ........66

Oxystomus $\ldots \ldots \ldots 71$

Oxyurichthys $\ldots \ldots \ldots 150$

Oxyurus ...........69

Oxyzygonectes $\ldots \ldots 80$

Ozodura ....................

Ozorthe ..........131

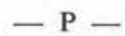

Pachycara .........133

Pachycarichthys ....133

Pachychilon

S. $1866 \ldots \ldots \ldots . .51$

Pachychilon

S. $1882 \ldots \ldots \ldots .51$

Pachygnathus ....... 138

Pachylabrus ........ 119

Pachymetopon ......114

Pachynathus ......138

Pachypanchax .......880

Pachypops ......... 109

Pachypterus .......61

Pachystomias .......28

Pachystomus .......51

Pachytrigla ........156

Pachyula ..........56

Pachyurus Agsz. ..... 109

Pachyurus Swsw. .....67

Рacu ...........442

Padogobius .......... 150

Paeneapocryptes ....150

Pagellus ...........111

Pagetodes ...........126

Pagetopsis ........126

Pagothenia ......... 127

Pagrichthys .........111

Pagrosomus .......111

Pagrus (P.)

LACPD.

Pagrus Cuv. ............111

Palaenichthys ....... 156

Palamita ..........140

ANN. PARASITO. HUM. ET COMP., 1962, 37, $\mathrm{n}^{\circ} 6^{\text {bis }}$ (fasc. suppl.) 
Paleatogobius ......150

Palinurichthys BLKR. . 143

Palinurichthys GILL ...143

Palinurus ..........143

Pallasia ..........36-163

Pallasina ...........163

Pallidogobius .......150

Palmichthys ........78

Paloa ..............146

Palometa .........142

Palosoma .........81

Palsartia ..........92

Palunolepis ........119

Palutrus ..........150

Pama ..............101

Pammelas .........143

Pampanoa .........103

Pamphoria ........81

Pamphorichthys .....81

Pampus BPTE ......142

Pampus B. FwlR. . . 142

Panamichthys ......81

Panaque ..........66

Panchax ..........80

Pandaka ............150

Pangasianodon ......61

Pangio .............55

Pangasius ..........61

Panichthys .........157

Panotus ...........155

Pantanodon .........80

Pantodon ..........29

Pantolabus ..........103

Pantophos ..........32

Pantosteus .........45

Panturichthys ......67

Papenua ...........150

Papillopogon ........99

Papillocheilus .......51

Papilloculiceps .....159

Papuservus ..........108

Papyrichthys .......143

Papyrocephalus .....84

Parabagrus ........63

Parabalistes ........138

Parabarbus ........51

Parabarilius .........51

Parabassogigas .....135

Parabathymyrus .....70

Parabelonichthys ....83

Parabembras ........159

Parablennius ........128

Parabodianus ........109

Parabothus .........166

Parabrachirus ......155

Parabramis ........51

Parabranchioica .....64

Parabrotula .........135

Paracaesio ..........107

Paracallionymus ....127
Paracanthobrama ....51

Paracanthochaetodon .115

Paracanthopoma ....64

Paracanthostracion ....33

Paracara ...........117

Paracentropogon ...155

Paracentropristis ....92

Paracentroscyllium ....13

Paraceratias ........172

Paracetopsis (G.)

BLKR.

Paracetopsis

EGMN. et BN. ......64

Parachaenichthys ....126

Parachaeturichthys ...150

Paracheilognathus ....51

Parachela ..........51

Parachromis AGsz. . . 117

Parachromis REgAN .....

Paracirrhites BLKR. ...118

Paracirrhites

STDChNR, et DöDLN. .118

Paracitharus .......166

Paraclinus .........129

Paracobitis ........55

Paraconodon .......107

Paracottus ..........161

Paracristiceps .......130

Paracrossocheilus ......51

Paracrossochilus ......51

Paracubiceps ........143

Paracyttopsis .......90

Paradanio .........51

Paradentex .............106

Paradicichthys ......105

Paradicrolene .......135

Paradicula ..........169

Paradiodon ............35

Paradiplomystax .....56

Paradiplomystes .....56

Paradiretmus ........88

Paradistichodus .....43

Paradoxichthys .....73

Paradules BLKR. ......95

Paradules KLZGR. ....995

Paragalaxias ........26

Paragaleus ..........12

Paragambusia .......81

Paraglyphidodon ....120

Paragobiodon .......150

Paragobioides .......152

Paragobiopsis .......150

Paragobius .........150

Paragoniates ........40

Paragonus GILL .....163

Paragonus GCHNT. ...163

Paragonus RIBRo. ...163

Paragyrops ..........111

Parahalecula .......22

Paraheminodus ......156
Parahemiodon ......66 66

Parahollardia ....... 138

Parahynnodus .......99

Parailia .........6.61

Parajulis ..........123

Parakuhlia .........95

Parakysis .........60

Paralabidochromis . .117

Paralabrax ........92

Paralarimus ...........109

Paralaubuca .........51

Paralepidocephalus ....55

Paralepis ...........31

Paraleucogobio ......51

Paralichthodes .......168

Paralichthys .......166

Paralimanda .......167

Paraliparis ..........164

Paralonchurus ......109

Paralosa BLKR. ......22

Paralosa Regan ......22

Paraluteres .........139

Paralycodes .........133

Paramacrurus ......84

Parambassis ........97

Paramia ...........99

Paramicrophis ......83

Paraminous ........155

Paramisgurnus .......55

Paramonacanthus

BLKR. .......... 139

Paramonacanthus

STDCHR. ..........139

Paramphilius .......60

Paramyloplus ......40

Paraphiprion ........120

Paramyomyrus .....29

Paramyrus .........70

Paramyxine ......... 7

Paranandus ........115

Parancistrus ........56

Paraneetroplus .....117

Paranotropis ........51

Paranthias ........92

Parapegasus ........165

Parapelecus .........51

Parapempheris ......998

Parapercichthys ....125

Parapercis BLKR. ...125

Parapercis

STNDCHR. .......125

Parapetenia ........117

Paraphago .........43

Paraphotichthys ....27

Paraphoxinus .......51

Paraphractura ......6.60

Paraphya ..........150

Parapimelodus .....6.63

Paraplagusia ........170

Paraplesichthys ......101 


\begin{tabular}{|c|c|c|}
\hline Paraplesiops ......996 & Paratylognathus & Parvacara \\
\hline Paraploactis ......159 & Paratyntlastes ..... & Parviclinus $\ldots \ldots \ldots$ \\
\hline Paraplotosus & Parauchenipterus ....55 & Parvicrepis .......... \\
\hline Parapocryptes. & Parauchenoglanis & Parvigobius ....... \\
\hline Parapoecilia ... & Paravandellia $\ldots \ldots .64$ & Parviparma $\ldots \ldots \ldots$ \\
\hline Parapomacentrus & xenomystax $\ldots \ldots 68$ & Parvoraja ........ \\
\hline Parapriacanthus & Parazenopsis .......90 & Paschalestes ....... \\
\hline Parapristipoma : & achirus & Passer KLEIN \\
\hline Parapristiurus .... & Parecbasis ........40 & Passer (K.) Valcs . \\
\hline Paraprocypris .....51 & Parectodus ........ 117 & inaca DEKAY .... \\
\hline Paraprotomyzon & don & ca Gronow .. \\
\hline Paraprotosalanx & haphis $\ldots \ldots 66$ & nacae $\ldots \ldots \ldots$ \\
\hline Parapsenes ...... & hina $\quad \ldots \ldots 66$ & nachus $\ldots \ldots \ldots$ \\
\hline ettus ... & lemaria . & cus $\ldots \ldots \ldots$. \\
\hline decheneis & ippus ... & cea $\ldots \ldots \ldots$ \\
\hline Parapsilorhynchus & $s \ldots \ldots$ & oscirtes ..... \\
\hline pterois .... & .110 & clinus ...... \\
\hline terygotrigla & 117 & $\ldots \ldots$ \\
\hline asbora .... & iloglanis ... & lopesia .. \\
\hline ops ....... & mias $\quad \ldots$. & Pedalibrycon .... \\
\hline rhodeus .... & pius . . & lion $\ldots \ldots \ldots$ \\
\hline rhynchobdella & $\ldots 75$ & Pedites \\
\hline rius ...... & 75 & $u s, \ldots \ldots$ \\
\hline .160 & $u m$ & dictis $\ldots$ \\
\hline utilus & & $\mathrm{Pe}_{2}$ \\
\hline Parasalanx & ictylus & ocyclus \\
\hline irhynchus & $\begin{aligned} 55 \\
60\end{aligned}$ & Pelamichthys. \\
\hline othorax & 0 & $\begin{array}{l}P \\
P\end{array}$ \\
\hline 107 & 139 & $\begin{array}{l}P \\
P\end{array}$ \\
\hline brops & .64 & $P e$ \\
\hline ena & glossus & anichthys \\
\hline enodes & $63-64$ & inomimus \\
\hline 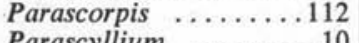 & opterus . . & $u s \ldots \ldots$ \\
\hline um & .106 & hhus. \\
\hline mer & aemeria & grinia \\
\hline Pa & .120 & olus \\
\hline & aturus .. & iromis \\
\hline chthys & husus & $\mathrm{Pe}$ \\
\hline thias & .155 & Pell \\
\hline ops & $\ldots 43$ & Pellonulops : \\
\hline nonotus & ontaspis . & \\
\hline philus & lontops .... & atochromis \\
\hline as $\ldots$. & sarcus. & lichthys . \\
\hline$m a \ldots$ & 103 & Pelopsia ... \\
\hline tes. & rodes. & Pelo \\
\hline ateus & codes... & Pelc \\
\hline oma & .136 & $P$ \\
\hline & halus & tis \\
\hline nathus & .167 & rtia .. \\
\hline ophorus & 103 & \\
\hline pus. & & ops \\
\hline Paratherina & inthias & obagrus \\
\hline .140 & hromenus & larpodon. \\
\hline & $a$. & amphus \\
\hline ichthys & clus & $P$ \\
\hline$P a$ & ichthys & herichthys \\
\hline Inou & 150 & $P$ \\
\hline Paratrigla & thys .. & urus \\
\hline Paratrygon .........10 & Parupeneus $\quad \ldots \ldots \ldots 11$ & Penetropteryx $\ldots \ldots$ \\
\hline
\end{tabular}




\begin{tabular}{|c|c|c|}
\hline & & \\
\hline enicelinus & & agoniates \\
\hline $\begin{array}{l}\text { enicipelta } \ldots \ldots \ldots \\
\text { ennahia }\end{array}$ & escadorichthys. & Pherallodiscus ..... \\
\hline $\begin{array}{l}\text { Pennahia } . \\
\text { Pennon } . .\end{array}$ & Petacara $\ldots \ldots \ldots$ & $\begin{array}{l}\text { Phillippsichthys .... } \\
\text { Philypnoides }\end{array}$ \\
\hline 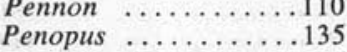 & $\begin{array}{l}\text { Petalichthys } \ldots \ldots \ldots 74 \\
\text { Petalosoma }\end{array}$ & $\begin{array}{l}\text { Philypnoides } \\
\text { Philypnodon }\end{array}$ \\
\hline Pentaceropsis .......115 & Petalurichthys .......81 & Philypnus. \\
\hline aceros ... & Petenia & Phobetor \\
\hline Pentanchus .. & Petersialestes .......40 & Phocaegadus \\
\hline Pentanemus. & Petersius & Pholidapus \\
\hline entapodus ... & Petimbuabo .... & Pholidichthys \\
\hline Pentaprion ..........106 & $\ldots \ldots \ldots 130$ & Pholidus \\
\hline ipus ... & Petrocephalus ... & Pholis SCOP. \\
\hline roge. & Petrochromis ... & Pholis GRONOW. \\
\hline Pentherichthys & Petrognathus GIRD. ..130 & Pholis RösE ..... \\
\hline Peprilus .... & Petrognathus $(G)$. & Pholis Cuv. \\
\hline Perca & et Evmn. . . & Pholis Cuv. et VAL. \\
\hline -labrax & Petrometopon ......94 & Phosichthys ....... \\
\hline$a x \rightarrow$ & $\ldots \ldots \ldots$ & Photoblepharon \\
\hline 5 & 124 & Photocorynus \\
\hline lates ... & Petroschmidtia ... & enis \\
\hline$a \ldots$ & scirtes $\ldots . .$. & $P h$ \\
\hline hias & Petrotilapia ........117 & Photonectoides \\
\hline rinodes & Petrotyx ......... & Photonectops \\
\hline ina.. & $\ldots \ldots$ & omias \\
\hline hthys . & $\ldots \ldots$ & $\ldots \ldots 21$ \\
\hline lia.... & Pfrille & Phoxargyrea \\
\hline $\mathrm{Pe}$ & don & nellus \\
\hline KLein & omonas ... & ...... \\
\hline Per & pogon $\ldots$ & Phoxinus RAFNSQ. . \\
\hline & onichthys . & us Agsz. . \\
\hline Percis S & $\ldots \ldots \ldots \ldots \ldots 43$ & $\ldots \ldots \ldots \ldots 51$ \\
\hline Percis (S.) $\mathrm{RA}_{\mathrm{A}}$ & borus ...... & psis \\
\hline cypris & .61 & ephalus \\
\hline his & 29 & iemus. \\
\hline sis ... & romacrurus ... & $P$ \\
\hline oma & onotus ... & hthys .. \\
\hline tus $\ldots$ & istes $\ldots .$. & tobius $\ldots . .$. \\
\hline & $\ldots$. & $\ldots$. \\
\hline mato & hynus ... & 171 \\
\hline pus & hys & thys \\
\hline & .81 & mbus \\
\hline hthalmo & tus $\ldots .$. & $P$ \\
\hline hthalmu & chus ... & thys \\
\hline & thus ... & ÖSE \\
\hline us & nus $\ldots \ldots . .81$ & \\
\hline & ates $\ldots$ & CHNDR. \\
\hline idium & ranchus. & \\
\hline hion & halus. & $\ldots \ldots 133$ \\
\hline & $\ldots \ldots$ & $y s \quad \ldots \ldots$ \\
\hline inous & keus & yra \\
\hline sia & $87-96$ & $m m a$ \\
\hline & 161 & \\
\hline edys ... & tostoma ... & Phyllophichthus \\
\hline era & chthys & Phyllophorus \\
\hline & .51 & teryx \\
\hline nichthys & Phenacobrycon ... & Physailia \\
\hline & gaster ... & Physiculus \\
\hline & mus & odon \\
\hline Pertica ..... & oscorpius ......155 & Physogaster \\
\hline Perugia & Phenacostethus & Physopyxis \\
\hline
\end{tabular}


Phytichthys

Piabarchus

Piabina

Piabuca ............. 40

Piabucidium ........440

Piabucina .........40

Piabucus .........440

Piaractus .........440

Picorellus ..........30

Pictiblennius .......129

Pictilabrus .........123

Piescephalus .......153

Pigus ...........51

Pikea ...........994

Pileoma ..........100

Pilodictis .........59

Pimelepterus .......113

Pimelodella ........63

Pimelodina ........6.63

Pimelodus .........63

Pimelometopon ......121

Pimelonotus .......63

Pimelotropis .......63

Pimephales ........51

Pingalla .........92

Pinguilabrum ......992

Pinguipes ........125

Pinirampus .......63

Pinjalo ..........107

Pinnacorvina .......109

Pintoichthys .......117

Pipetella ..........83

Pipettella ........83

Pipidonia ........150

Piramutana .......6.63

Pirarara ..........63

Piratinga ........663

Pirellinus ........136

Pirene ...........120

Pisciregia .........78

Pisodonophis .......71

Pisodontophis ......71

Pisoodontophis .....771

Pithecocharax ......42

Pithecomyzon .....445

Placopharynx ......45

Plagiogeneion ...104-108

Plagiognathops .....552

Plagiognathus .......52

Plagiogrammus .....132

Plagiopsetta .......166

Plagioscion ..........109

Plagiotremus ......132

Plagiusia .........170

Plagopterus ........52

Plagusia Browne ... 170

Plagusia RAFNSQ. ... 170

Plagusia (B.) Cuv. . . 170

Plagusia SwnsN. ..... 170

Plagusia BPTE. ...... 170
Plagyodus PLLs.

Plagyodus (S.) GNTHR. . 32

Planaltina ........40

Planctanthias ......92

Planerocephalus .....16

Planiliza ..........77

Planiprora ..........159

Plargyrus .........52

Platacanthus .......55

Plataplochilus ......80

Platax ............ 112

Plataxoides ........117

Platea ............ 133

Platessa ........... 167

Platichthys .........167

Platiglossus ........123

Platirostra ..........19

Platistus .........64

Platophrys ........166

Platopterus ........ 15

Platotichthys ....... 166

Platuronides ......72

Platybelone ........74

Platyberyx ........88

Platycanthus .........33

Platycara .........5 52

Platycephalops .....146

Platycephalus RIBRo ..95

Platycephalus BL. ...159

Platychoerops .......121

Platycottus ......... 162

Platydoras ........57

Platygaster .........23

Platyglossus ...... 123

Platygobio .......52

Platygobius ....... 150

Platyinius ....................

Platylepes ......... 102

Platynematichthys ...63

Platypanchax .......80

Platypharodon ......55

Platypodon ..........12

Platypodus ......... 144

Platypoecilus .......8.81

Platypogon .......6.63

Platypornax ........... 14

Platyptera ......... 144

Platypterus ........155

Platypus ..........144

Platyrhina ......... 15

Platyrhinoidis ......15

Platysilurus .......63

Platysoma .........95

Platysomatichthys ...167

Platysome ........95

Platysomus .........103

Platysqualus .......12

Platystacus KLeIN ...664
Platystacus $(K$.

BL. ........63-64

Platystethus .......142

Platystoma .......6.63

Platystomatichthys ...63

Platystomatos ......6.63

Platytaeniodus ....... 117

Platytroctegen .......21

Platytroctes ........21

Platytropius .......61

Platyuraspis ........ 104

Plecodus ............117

Plecoglossus .......25

Plecopodus ........ 152

Plecostomus Gronow .66

Plecostomus (G.)

BLKR. . ......66 66

Plectobranchus ....132

Plectorhinchus .....107

Plectorhynchus .....107

Plectranthias .......92

Plectrochilus .......64 64

Plectrogenium .....155

Plectroglyphidodon ..120

Plectromus ......... 89

Plectroperca ........994

Plectrophallus ......81

Plectroplites ........994

Plectropoma .........94

Plectropomus ......994

Plectrostethus ......76

Plectrostoma ........10

Plectrypops ........89

Plesioperca ........ 100

Plesiops ..........96

Plethodectes ........40

Pleuracromylodon ...12

Pleuragramma ...... 127

Pleuranacanthus .....35

Pleurogadus .......86

Pleurogobius ....... 150

Pleurogrammus .....158

Pleurolepis ......... 100

Pleuronectes ........167

Pleuronichthys .....167

Pleuroperca ........994

Pleurophysus ......64 64

Pleuroscopus ......126

Pleurosicya ........150

Pleurosicyops ....... 150

Pleurothyris .......27

Pleurothysis .......27

Plicomugil .........77

Pliosteostoma .......23

Pliotrema ........... 14

Plitops ............ 115

Plotosus .........58

Pluchus .......... 107

Plumantennatus .....171

Pluto .......... 72 


\begin{tabular}{|c|c|c|}
\hline etta & mus & tus \\
\hline Pneumabranchus & Polydactylus .. & Poropuntius \\
\hline neumatophorus : & Polyipnus. & Porotergus \\
\hline Pnictes $\ldots \ldots \ldots \ldots$ & Polymetone ...... & Porteridia \\
\hline Poblana ......... & Polymixia & Porthmeus .... \\
\hline Podabrus .......... & Polynemus LinNé & Potamalosa \\
\hline Podager ............110 & Polynemus GILL... & Potamocottus \\
\hline Podateles ......... & Polyodon LACPD. .....19 & Potamophylax ..... \\
\hline Oodoleptus ....... & olyodon & Potamorhina .... \\
\hline Podonema ........ & BL. et SCHNDR. & Potamorrhaphis \\
\hline Podothecus ........163 & Polypera .........164 & Potamothrissa \\
\hline$\ldots \ldots \ldots$ & Polyprion & Potamotrygon ...... \\
\hline Poecilichthys ..... & Polyprosopus .... & Poutawa \\
\hline tes $\ldots \ldots \ldots$ & chthys ... & ahipokia \\
\hline ycon ... & Polypterus ...... & rmosania \\
\hline ephalus & Polysteganus $\ldots 1$ & Pranesella ...... \\
\hline Poecilocharax ......440 & Polyuranodon ......6.67 & Pranesus. \\
\hline Poeciloconger ......669 & mpsis ... & Premnas \\
\hline Poecilodes ........ & Pomacanthodes .. & Prenes.. \\
\hline is $\ldots \ldots$...... & Pomacanthops .. & nthichthys \\
\hline setta $\ldots . .$. & Pomacanthus .... & nthopsis. \\
\hline sis ..... & ntrus . & ithus \\
\hline$n a \ldots \ldots$ & lasyina ... & lla. \\
\hline natops ... & dasys ....... & ichthys .... \\
\hline issa ... & Pot & \\
\hline hthys .. & aprion $\ldots$ & ila \\
\hline$\ldots \ldots$ & Pomatias .......... & tus \\
\hline tris .... & Pomatias & pina . \\
\hline Po & Pomatias & don. \\
\hline thys ....... & oichthys & Iraco. \\
\hline $\operatorname{arax} \ldots$ & Us LACPD. & \\
\hline lius ...... & is $(R$.) & ophis \\
\hline athus .. & 9 & atichthys \\
\hline obius .. & Pon & \\
\hline $\mathrm{Po}$ & schistus & .12 \\
\hline macheilus & don ....... & tius \\
\hline rca $\ldots . .$. & Pon & ama \\
\hline $\mathrm{Pog}$ & Pomotis RAFNSQ. & butis ... \\
\hline iryne .. & Pomotis Cuv. ..... & des ..... \\
\hline & Por & \\
\hline oides. & us LowE & epis \\
\hline pius ... & ilus MNDS. & parus \\
\hline pis ... & Po & \\
\hline & .150 & .137 \\
\hline . & us $\ldots \ldots$ & lichthys \\
\hline $\mathrm{Po}$ & & \\
\hline nius & 111 & \\
\hline us $\quad . .$. . & & plichthys \\
\hline ius ... & 3 & otrigla \\
\hline ema .... & 29 & cistrus \\
\hline & chus. & ogon \\
\hline & lus. & \\
\hline honotus & nus $\ldots \ldots \ldots \ldots 131$ & harax .. \\
\hline & & \\
\hline odon & & ister \\
\hline nthus .. & .135 & enys... \\
\hline & bius ....... & \\
\hline opsis & & doryrhamphus \\
\hline Polycentrus ..... & 35 & Pristiophorus \\
\hline Polycirrhus ... & a & Pristiopsis \\
\hline
\end{tabular}


Pristipoma ..........107

Pristipomoides .....106

Pristipomus ........107

Pristis KLeIN .......15

Pristis LINCK ........15

Pristis Lтнм. ........15

Pristiurus M. et $\mathrm{H}$. ...13

Pristiurus BPTE .....13

Pristobrycon ......440

Pristocantharus .....107

Pristolepis ........115

Pristotis ...........120

Proamblys ........106

Probarbus .........55

Probolodus ........440

Procatopus ........80

Proceros ...........19

Prochilodus ........42

Prochilus KLEIN ......120

Prochilus Cuv. ......146

Procottus ......161-162

Proctomyctophum ...32

Proctostegus ........90

Procypris .........52

Proditor ...........21

Prodontocharax .....40

Proeutropichthys .....61

Proeutropius ........61

Profundisudis .......31

Profundulus ........80

Progastromyzon .....55

Prognathodes .......115

Prognichthys ......75

Prognurus ........ 164

Programmus .......132

Prolabeo ...........552

Prolabeops .........552

Prolatilus .........101

Promacheon ........32

Promecocephalus .....35

Promecococephalus ....35

Promerluccius .......85

Prometheichthys .....141

Prometheus ........141

Promicrops ........994

Promicropterus ......94

Promitrella ........89

Promyllantor ......669

Pronotogrammus ....92

Proodus ............35

Prophagorus .......61

Propoma ..........107

Proportheus ......440

Propseudecheneis ....60

Propterygia .........15

Proscyllium .........13

Proscymnodon ......13

Prosoarchus ........26

Prosopium .........25

Prosoplismus
Prosopodasys .......155|Pseudaphritis .....127

Prospinus .........94 Pseudaphya ....... 150

Prostheacanthus .....55 Pseudapocryptes .... 150

Proteracanthus .....114 Pseudariodes .......63

Proterorhinus .......150 Pseudarius .........56

Prostistius ........78 Pseudaspius ......52

Protoblepharon ......32 Pseudauchenipterus ...57

Protocampus .......83 Pseudaustroglossus ...169

Protomelus .......173 Pseudecheneis ......60

Protomyzon ........55 Pseudechidna ......67

Protoporus .........52 Pseudepapterus .....557

Protopsetta ..............57 57

Protopterus .......173 Pseuderythrinus ....40

Prototroctes .......26 Pseudetroplus ......117

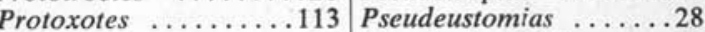

Protozygaena .......12 Pseudeutropichthys ...59

Prymnothonoides ....31 Pseudeutropius .....61

Prymnothonus .......31 Pseudoaesopia ...... 169

Psalidodon ........440 Pseudoambassis ......97

Psalidostoma .......40 Pseudobagrichthys ...59

Psalisostomus .......19 Pseudobagrus

Psammichthys ..150-152 $\quad$ B. $1858 \ldots \ldots \ldots$

Psammobatis ........15 Pseudobagrus

Psammobatus ......15 B. $1860 \ldots \ldots \ldots$

Psammodiscus ........ 168 Pseudobalistes ......138

Psammogobius .....150 Pseudobarbus ......52

Psammoperca ......95 Pseudobatrachus .... 153

Psapharocetus ......89 Pseudoblennius .....161

Psectrogaster ......442 Pseudobrama ,.....52

Psednoblennius .....130 Pseudobythites .....133

Psednos ........... 164 Pseudocalophysus ...6.63

Pselaphias .........150 Pseudocaranx ....... 104

Psellogrammus .....40 Pseudocepola .......126

Psenes ................643 64

Psenopsis ...........142 Pseudochaenichthys ..126

Psephurus .........19 Pseudochalceus .....40

Psetta KLEIN ........166 Pseudocheilinus .....123

Psetta Swns. .......166 Pseudocheirodon .....40

Psettias ...........112 Pseudochirocentrodon .23

Psettichthys ........167 Pseudochromichthys .996

Psettina .........166 Pseudochromis .....96

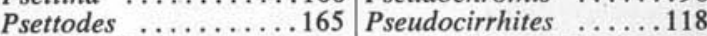

Psettus (C.) Pseudocitharichthys . 166

LACPD. ........112 Pseudoclinus ......161

Psettus (C.) Pseudocoris ........123

Cuv. ........112 Pseudocorynopoma ...40

Psettylis ........... 166 Pseudocrenilabrus ...92

Pseudacanthicus ....66 Pseudoculter .......52

Pseudageneiosus .....57 Pseudocurimata ....42

Pseudalphestes .....994 Pseudocyttus .......99

Pseudalutarius ......139 Pseudodax .........121

Pseudaluteres .......139 Pseudodon ...........55

Pseudaluterius ......139 Pseudodoras .......57

Pseudambassis ......997 Pseudoechidna .....67

Pseudamia ........99 Pseudogastromyzon ...55

Pseudamiatus .......99 Pseudogobio .......52

Pseudamiops .......99 Pseudogobiodon ....150

Pseudanampses ..... 123 Pseudogobiops .......52

Pseudancistrus ......66 Pseudogobiopsis ....150

Pseudanthias .......992 Pseudogobius .......150 


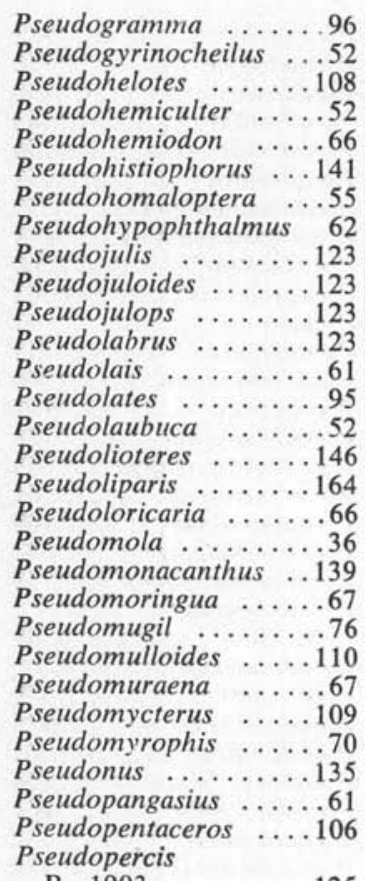

R. $1903 \ldots \ldots \ldots 125$

Pseudopercis

R. $1918 \ldots \ldots \ldots 117$

Pseudoperilampus .....52

Pseudophallus .......83

Pseudophichthys ....6.69

Pseudophidium ......136

Pseudophoxinus ......52

Pseudophycis ......86

Pseudopimelodus .....66

Pseudoplatichthys ...167

Pseudoplatystoma ....63

Pseudoplesiops

B. $1858 \ldots \ldots \ldots 96$

Pseudoplesiops

B. 1899 ... . . . 117

Pseudopleuronectes ...167

Pseudopoecilia ......881

Pseudopomacentrus ..120

Pseudopriacanthus ...998

Pseudopristipoma ...107

Pseudopsectrogaster ...42

Pseudopterus KLEIN ..155

Pseudopterus (K.)

BLKR. $\quad \ldots \ldots \ldots \ldots 155$

Pseudopuntungia ......52

Pseudoraja .........15

Pseudorasbora ......55

Pseudorhamdia .....663

Pseudorhombus .......166
Pseudorinelepis ......66

Pseudosawara ........140

Pseudoscapirhynchus ..18

Pseudoscarus ........124

Pseudosciaena .......109

Pseudoscopelus .....127

Pseudoscymnus ......13

Pseudosebastes .....155

Pseudoserranus ......92

Pseudosilurus ........58

Pseudosphromenus ...144

Pseudostegophilus ...664

Pseudosynanceia .....157

Pseudosyngnathus .....83

Pseudosynodontis ....66

Pseudothyrina ......78

Pseudotocinclus .....66

Pseudotolithus ........109

Pseudotrachinus .....125

Pseudotriacanthus ...138

Pseudotriakis ..........10

Pseudotropheus ......117

Pseudotrypauchen ....150

Pseudotylosurus .....74

Pseudoxenomystax ...68

Pseudoxiphophorus ....81

Pseudupeneus ......110

Psilocephalus ........139

Psilocranium .......118

Psilodraco .........126

Psiloides ...........146

Psilonotus ..........34

Psilopentapodus ....110

Psilorhynchus .......55

Psilosomus .........152

Psilotris ..........146

Psilus .............146

Psittacus ...........124

Psychichthys .......18

Psychrolutes .......162.

Psychromaster .....100

Psychropoecilia .....81

Ptarmus ...........155

Ptax .............141

Ptenichthys .......75

Ptenonotus ........75

Pteraclidus .........91

Pteraclis G. 1763 ....91

Pteraclis G. 1772 ....91

Pteragogus .........121

Pteranthias ........92

Pterapogon ........999

Pterapon ..........108

Ptereleotris .........146

Pterengraulis ........24

Pterichthus ........75

Pterichthyodes .....155

Pterichthys Swns. ....155

Pteridium Scop. .....91
Pteridium

FIL. et Very .....86

Pterobrycon ........40

Pterobunocepha!us ...663

Pterocaesio ......... 107

Pterocapoeta .......52

Pterocephala ........17

Pterocryptis ........58

Pteroculiops .......146

Pterocyclosoma .....120

Pterodicromita ......135

Pterodiscus ........41

Pterodoras .........57

Pteroglanis PeARs. ...663

Pteroglanis FWLR. ...660

Pterogobius .........150

Pterogymnus ........111

Pterohemiodus ...440-43

Pteroidichthys

B. $1876 \ldots \ldots \ldots 155$

Pteroidichthys

B. $1856 \ldots \ldots \ldots 155$

Pteroidonus ........135

Pterois ...........155

Pterolamia BRNG. . . . 12

Pterolamia SPRGR. ....12

Pterolamiops ........12

Pterolebias ........80

Pteroleptus .........155

Pteromugil ........77

Pteromylaeus .......17

Pteronemus ........119

Pteronotus .........63

Pteropangasius .....6.61

Pteropelor .........155

Pterophryne .......171

Pterophrynoides .....171

Pterophrynus ........171

Pterophyllum .......117

Pteroplatea .........16

Pteroplatytrygon ......16

Pteropodus ........155

Pterops ..........146

Pteropsarion .......52

Pteropsaron .......125

Pteropsoglanis ......60

Pteropterus .......152-155

Pterorhombus ......142

Pteroscion .........109

Pterosmaris .......112

Pierothrissus .......20

Pterotolithus .......101

Pterotyx ...........133

Pterozygus ........55

Ptérure ...........83

Pterurus RAFNSQ. ....71

Pterurus Swnsn. .....67

Pterycombus .......91

Pterygiocottus ......160

Pterygoplichthys .....66 
Pterygotrigla .......157

Ptilichthys ........131

Ptychidio ........52

Ptychobarbus .......52

Ptychecheilus ......52

Ptychochromis .....118

Ptycholepis .......21

Ptychostomus ......45

Ptyobranchus .....68-71

Ptyonotus ........161

Pungitius ..........75

Pungnaso .........83

Pungtungia .......55

Puntazzo $\ldots \ldots \ldots \ldots 111$

Puntioplites .........52

Puntius .........52

Pusichthys ......661

Pycnochromis .....120

Pycnocraspedon ....135

Pycnomma ........150

Pygidianops .......64

Pygidium .........6.64

Pygocentrus .......40

Pygoplites .........115

Pygopristis .......40

Pygosteus Brvt. .....75

Pygosteus (B.) GILL . . 76

Pyramodon RDCLF. ..135

Pyramodon RGN. ...135

Pyrodon ...........138

Pyrrhulina ........440

Pythonichthys ......67

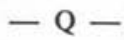

Quadrarius .......115

Quassilabia ........45

Quassinemus .......71

Quassiremus .......71

Quenselia .........169

Queriblennius ......129

Querigalaxias ......26

Querimana ........77

Quietula ...........150

Quincuncia ........85

Quinquarius .......115

Quintana .........80

Quirichthys .......78

Quiris .............78

Quisquilius ........ 150

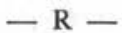

$R a$

Rabdolichops

Rabdophorus

Rabidus

Rabirubia $\ldots . . . . . . . . . .60$

Rabula .............67 67

Rachovia .........80
Rachoviscus .......40| Reighardina ........7

Rachycentron ......102 Reinhardtius ......167

Rachycentrum ......102 Relictogobius ...... 150

Racoma ........52 Remilegia ........ 154

Raconda ...........23 Remora CATsB. ..... 154

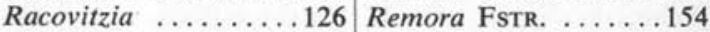

Radcliffella .......150 Remora GILL ......154

Radulinellus .......161 Remorina ........154

Radulinopsis .......161 Remoropsis .......154

Radulinus ........160 Rendahlia ....... 168

Rafinesquiellus .....100 Reniceps ..........12

Raia ..................... Repomucents

Raiamas ........52 Reporhamphus ......75

Rainfordia ........92 Repotrudis .......159

Raitaboura .......6.6. Retroculus ........ 118

Raitoboura .......68 Retropinna ........26

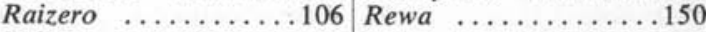

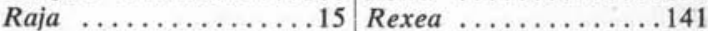

Rajabatis ..............4 Rh

Rama ..........59 Rhabdalestes ......440

Rambaibarnia ......52 Rhabdamia ........999

Ramirezella .......40 Rhaddoblennius ....129

Ramphistoma .....74 Rhabdolichops .....44

Ramphistoma .....74 Rhabdopetersius ....40

Ramularia ........166 Rhabdophorus ....115

Raniceps ........86 Rhabdosargus .....111

Ranipterois .......155 Rhabdosebastes ...94-155

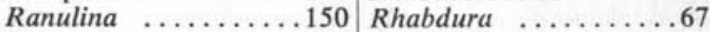

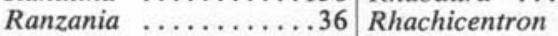

Raogobius ........150 Rhachinotus ......16

Raphiobelone ......74 Rhacochilus ......119

Raphiodontichthys ...40 Rhadinesthes .......27

Raphistoma ......74 Rhadinocentrus ....76

Rasbora .........52 Rhamdella .......63

Rasborella .......52 Rhamdia ........63

Rasborichthys ......52 Rhamdioglanis ....63

Rasborinus .......52 Rhamdiopsis .....63

Rastelliger ........140 Rhamnubia .......111

Rastineobola ......52 Rhamphichthys ....43

Rastrinus .......160 Rhamphobatis ....14

Rastrum ...............84 Rhamphoberyx

Rataboura ........68 Rhamphochromis ...118

Ratabulus ........159 Rhamphocottus ....162

Rathbunella ........125 Rhamphodermogenys .75

Recepoecilia ......80 Rhamphosternarchus ..44

Rectoris .........52 Rhaphiodon ......40

Redigobius ......... 150 Rhaphiolepis ....... 104

Regalecus BRnCH. ...87 Rhechias ........69

Regalecus AsCNS. ....87 Rhectogramma ......99

Reganella ........66 Rhegma .........97

Regania ..........85 Rhencus ............ 107

Reganichthys OGBY. ..105 Rheocles ........78

Reganichthys $\quad$ Rheocloides .......78

Bw. et BR. ....... 172 Rheocrypta ...... 100

Reganina ........40 Rheopresbe .......161

Reganisalanx .......25 Rhina B. et S. ........ 14

Reganochromis .....118 Rhina KLEIN ......14

Reganula .........172 Rhina RAFNSQ. .....14

Regificola .......... 104 Rhina $(K$.$) GILL ..... 14$

Regilophotes .......87 Rhincodon ........10 


\begin{tabular}{|c|c|c|}
\hline & & \\
\hline hinechidna & abotides $\mathrm{K}$ & Roccus \\
\hline Rhinelepis .......... & Rhombotides (K.) & Roeboides \\
\hline Rhineodon ........10 & BLKR. .......... & Roeboxodon ....... \\
\hline Rhinesomus ....... & Rhombus Cuv. .... & Roestes ...........440 \\
\hline Rhinichthys ... & Rhombus KLEIN ... & Rogadius ........ \\
\hline Rhinobagrus ...... & Rhombus LACPD. . & Rogenia .......... \\
\hline Rhinobatos & Rhonctscus ....... & Rohanus ..........52 \\
\hline batus LINCK & Rhothoeca & Rohita \\
\hline Rhinobatus B. et S. & Rhyacanthias ......992 & Rohitichthys .......55 \\
\hline Rhinoberyx ........89 & Rhyacichthys ......144 & Rohitodes \\
\hline Rhinobrycon $\therefore .$. . & Rhycherus & Rohtee \\
\hline Rhinochimaera ......18 & Rhynchactis ..... & Rohteichthys \\
\hline hinocryptis .......173 & Rhynchana ........30 & Romanichthys ......101 \\
\hline Rhinodactylus ..... & herus & Roncador .......... \\
\hline Rhinodon ..........10 & Rhynchias & Ronchifex ........121 \\
\hline doras .........57 & Rhynchichthys $\ldots \ldots 89$ & Rondeletia ...... \\
\hline Rhinoglanis ...... & Rhynchobatis .... & Ronquilus \\
\hline Rhinogobio ........ & Rhynchobatis ... & Rooseveltia \\
\hline bioides ......52 & batus $\ldots \ldots \ldots 14$ & eveltiella .... \\
\hline Rhinogobiops ......150 & Rhynchobdella ... & Rosanthias .... \\
\hline Rhinogobius .......150 & Rhynchoceratias ... & Rosaura ... \\
\hline aris ..... & onger ......69 & $R c$ \\
\hline ugil...$\ldots \ldots$ & Rhynchocymba ... & Rostrogobio .... \\
\hline aena ..... & Rhynchocypris ... & hleyia ... \\
\hline mus .......85 & adus ....... & ina JORDN. \\
\hline$\ldots \ldots \ldots$ & ohyalus .... & Rouleina FowLR. . \\
\hline tgusia $\ldots . .$. & agus .... & ella $\quad \therefore .$. \\
\hline chthys ..... & elates ......108 & $R u$ \\
\hline tera ..........17 & Iorhampus . & ralga $\ldots . .$. \\
\hline .15 & tomias ... & qualus. \\
\hline dinia .... & tracion ... & us ..... \\
\hline ion $\ldots \ldots \ldots 109$ & otus $\ldots . .$. & inatus $\ldots$. \\
\hline elus ..... & Rhy & $\ldots$. \\
\hline nnus ...... & .121 & $R$ \\
\hline lea .........169 & Rhytiodus & artes $\ldots$ \\
\hline is ...... & $\ldots \ldots 163$ & $g a \ldots$. \\
\hline cus ...... & nia CSTLN. .115 & lia .... \\
\hline$\ldots \ldots \ldots$ & Ric & ellia $\ldots . .$. \\
\hline iodon ... & & iops ... \\
\hline hthys. & nichthys & ius .... \\
\hline sia $\ldots \ldots \ldots \ldots 40$ & Richardsonius ......52 & iculus $\ldots . . . .$. \\
\hline des ... & $\ldots \ldots$ & us ... \\
\hline$\ldots .$. & & $R l$ \\
\hline & bius ... & Ruvettus ...........141 \\
\hline$\ldots \ldots \ldots 135$ & ius & Rypticus .........994 \\
\hline uriscus ... & $a \ldots \ldots \ldots \ldots 153$ & \\
\hline nenichthys ...132 & Rimicola .........153 & \\
\hline ractus ..... & & \\
\hline$R /$ & ...66 & ewia \\
\hline hirus ... & $\ldots \ldots \ldots 21$ & \\
\hline ocyttus .. & Rior & ranchus \\
\hline a $\ldots \ldots \ldots 104$ & $\ldots \ldots \ldots \ldots 151$ & derma ... \\
\hline & Rissola ...........136 & \\
\hline hthys & & \\
\hline lites ....... & Riukiuia & opharynx \\
\hline oides & Rivasella & \\
\hline anus & & stinus \\
\hline osolea $\quad . \ldots \ldots 168$ & chthys ..... & Sacura \\
\hline & Rivulus & \\
\hline
\end{tabular}


Sagamia ..........151

Sagamichthys ......21

Sagenichthys .......101

Sagittabarilius ......5 52

Salangichthys ......25

Salanx ...........25

Salar ...............24

Salaria ...........129

Salarias ..........129

Salarichthys .......129

Salarigobius ........151

Salariichthys .......129

Salema ...........111

Salilota .........86

Salminus ........44

Salmo ...........24

Salmoperca ........73

Salmophasia .......52

Salmostoma ........52

Salmothymus .......25

Salpa ...........106

Saltatrix ........... 102

Salvelini $\quad \ldots \ldots \ldots \ldots 24$

Salvelinus .........24

Samaris ............168

Samariscus .......... 168

Sambwa ..........52

Sanagia ..........52

Sandakanus ........76

Sandat ClQT. . ........101

Sandat B. DE ST. V. .. 101

Sandelia .............144

Sander ............101

Sandrus ...........101

Sanopus ..........153

Saraca ...........118

Saracara .......... 118

Sarchirus ..........19

Sarcidium ........52

Sarcina ..........142

Sarcocheilichthys ....552

Sarcodaces ........41

Sarda GRONOW

Sarda (P.) LACPD. . ..106

Sarda Cuv. .......... 140

Sardina ..........23

Sardinella .........23

Sardinia ..........23

Sardinops ..........23

Sargocentron ......899

Sargochromis ......118

Sargosomus ........119

Sargus KLeIn .......111

Sargus PlmR. .......106

Sargus Cuv. .......111

Sarotherodon ......118

Sarothrodus .......115

Sarpa ............114

Sarragia .........52

Sarritor .........163
Sartor $\ldots \ldots \ldots \ldots \ldots \ldots$
Satan $\ldots \ldots \ldots \ldots \ldots \ldots$
Satanoperca
Sathmonotus $\ldots \ldots \ldots \ldots$

Satsuma .........95

Satulinus .................

Satyrichthys ....... 157

Saurenchelys .......68

Saurida ........... 30

Sauridichthys ........ 30

Saurogobio .........55

Sauromuraenesox .....68

Saurus BRWN. ...... 104

Saurus Catsy. ...... 30

Saurus CUv. ......... 30

Sauvagea .......... 130

Sauvagella B. $1940 \ldots 23$

Sauvagella B. $1943 \ldots 23$

Sawara ............ 140

Saxilaga ..........26

Sayonara ..........92

Sayris ............74

Scaeops ...........166

Scaevius ..........106

Scalanago .........69

Scalicus ............157

Scaphesthes ........53

Scaphiodon .........53

Scaphiodonichthys ...53

Scaphiodontella ......53

Scaphiodontopsis .....53

Scaphirhynchops .....18

Scaphirhynchus ......19

Scaphognathops .....53

Scaphognathus ......53

Scapirhynchus ......19

Scardiniopsis .......53

Scardinius .........53

Scarichthys ........123

Scaridea .......123-124

Scarodon .........110

Scaroides ............ 124

Scarops ..........124

Scarostoma .......110

Scartelaos ..........152

Scartella ..........129

Scartes ...........129

Scartichthys ........129

Scartoblennius ....... 129

Scartomyzon ......45

Scarus FosKL. ......124

Scarus GRONOW ....121

Scarus (F.) BLKR. . . . 123

Scarus Cuv. ........123

Scatharus ......... 114

Scatophagus .........114

Sceptrias ..........999

Schacra ..........53

Schedophilopsis ......143

Schedophilus .......143
Schilbe ..........661

Schilbeichthys ......61

Schilbeodes ........660

Schilus ..........101

Schindleria ........136

Schipa ...........19

Schismatogobius ....151

Schismatorhynchos ....53

Schistoperca ...... 92

Schistorus ........94

Schistura ...........55

Schizochirus .......125

Schizocypris .......53

Schizodon .......442

Schizodontopsis .....442

Schizophallus ......81

Schizopyge .........53

Schizopygopsis ......53

Schizorhiza ........15

Schizothorax ......53

Schmidtia ......... 160

Schmidtina ......... 160

Schour ..........110

Schraitzer ..........101

Schubotzia ..........118

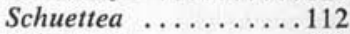

Schultzea ..........104

Schultzidia .........71

Schwetzochromis ....118

Sciadeichthys .....56-63

Sciadeoides ........63

Sciadeops ...........56

Sciades .........56-63

Sciadonus ......... 135

Sciaena LINNÉ ........ 109

Sciaena Jd et EvmN. . . 109

Sciaenoides (S.)

RHDSN. ........118

Sciaenoides BLYTH ...119

Sciaenops ..........109

Sciaenus ..........106

Scianectes .............. 166

Scidorhombus ......166

Scimnus .......... 14

Scissor .........44

Sclerocottus ........126

Sclerognathus .....445

Scleromystax ......6.65

Scleronema ........6.64

Scleropages .........29

Scleropteryx .........134

Sclerotis ...........97

Scobatus ..........15

Scobinichthys .......138

Scolecosoma .........7

Scolenchelys .......70

Scoliodon ........... 12

Scoliostomus .......21

Scolopacichthys .......74

Scolopsides .........107 
Scolopsis . . . . . . . . 108
Scomber . . . . . . .

Scomberoides ........104

Scomberomorus ......140

Scomberopsis ........53

Scombralabrax ......99

Scombresox ........74

Scombrocottus .....158

Scombrocypris ......53

Scombrolabrax ...999-141

Scombrops .........999

Scopas BPTE. ......137

Scopas (B.) KNER ....137

Scopelapogon .......99

Scopelarchoides ........32

Scopelarchus .........32

Scopelengenys .......32

Scopeloberyx .......89

Scopelogadus ........89

Scopelogenys ........32

Scopelopsis ........32

Scopelosaurus ........30

Scopelus ............32

Scophthalmus .......166

Scorpaena ........155

Scorpaenella ........155

Scorpaenichthys

BLKR. ..........155

Scorpaenichthys

GRD. .......155-160

Scorpaenodes .......155

Scorpaenopsella ....155

Scorpaenopsis .......155

Scorpichthes .......162

Scorpiodoras .......57

Scorpis .........112

Scorpius ..........155

Scortum ..........108

Scrofaria .........142

Scrophicephalus .....29

Scurrilichthys ......139

Scutengraulis .......24

Scutia ............67

Scuticaria .........67

Scyliorhinus ........13

Scylliogaleus ........12

Scyllium ..........13

Scymnodalatias ......14

Scymnodon ..........13

Scymnorhinus .......14

Scymnus .........14

Scyphius .........83

Scyris ..............104

Scytalichthys .......71

Scytallurus ........71

Scytalophis ........71

Sealeina ..........41

Searsia ...........21

Sebastapistes .......156

Sebastavus ........156
Sebastella

156 Serranochromis

118

Sebastes ...........156

Sebastichthys .......156

Sebastinus .........156

Sebastiscus ..........156

Sebastocarus .......156

Sebastocles ..........156

Sebastodes ..........156

Sebastoessus .......156

Sebastolobus .......156

Sebastomus ........156

Sebastoplus ..........156

Sebastopsis GILL ....156

Sebastopsis Svge. ....156

Sebastopyr .........156

Sebastosemus ......156

Sebastosomus ........156

Sectator ..........113

Securicula .........53

Secutor ..........106

Segutilum ........113

Selache ............10

Selachophidium ....133

Selanonius ..........10

Selar ...............104

Selaroides ..........104

Seleima ..........113

Selenanthias .......92

Selenaspis ..........56

Selene ............104

Selenia .............104

Selenotoca ..........114

Sema ............119

Semablennius ........129

Semachlorella .......123

Semadascyllus .......120

Semapagrus ........111

Semaprochilodus ......42

Semathunnus .......140

Semelcarinata ......42

Semicossyphus ......121

Semiculter .........53

Semilabeo .........55

Semiplotus ..........53

Semitapiscis ........42

Semotilus ..........53

Septobranchus .......57

Sericagobioides ......151

Seriola ............104

Seriolella ..........143

Seriolichthys ........ 104

Seriolina ............ 104

Seriolophus ......... 104

Seriphus A. $1854 \ldots 101$

Seriphus A. $1857 \ldots 109$

Serpa ..............32

Serranellus ........92

Serranguilla .......6.67

Serranichthys ......994

Serraniculus ........92
Serranops .........92

Serranus ........92-94

Serraria ...........100

Serrasalmo .......41

Serrasalmus .......41

Serrichromis .......120

Serrihastaperca ......92

Serrivomer ........72

Seserinus .........142

Setarches ..........156

Setipinna ..........24

Sewellia ...........55

Seychellea .........151

Shacra ...........53

Sheardichthys ......124

Shippofugu .........35

Shipwayia ........146

Shosaifugu .........35

Siaja ...........53

Siboma ...........53

Sicamugil .........77

Sicya ............151

Sicyases ...........154

Sicydiops .........151

Sicydium .........151

Sicyogaster BRNL. ....154

Sicyogaster GILL .....151

Sicyopterus ........151

Sicyopus ...........151

Sicyosus ..........151

Sidera ...........6.67

Siderea ..........67 67

Sierra .............140

Siganites ...........137

Siganus . . . . . . 137

Sigmistes ........162

Sigmops .........27

Sigmurus .........109

Signalosa .........23

Sikukia ...........53

Silhouettea ........151

Sillaginichthys .......101

Sillaginodes .........101

Sillaginopodys ......101

Sillaginopsis ........102

Sillago ............ 102

Silonia ..........61

Silonopangasius ....661

Silundia ..........61

Siluranodon .......61

Silurichthys ........58

Silurodes .........58

Siiurodon ..........58

Silurus ...........58

Silus ............26

Silvesterina .......69 69

Simenchelys ........66

Simobrama ........142
Sibogapistus .......156 
Simocantharus $\quad \ldots \ldots 114$
Simochromis

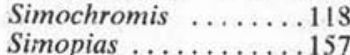

Simosyngnathus ....83

Simpsonichthys ......80

Sineleotris ..........146

Sinibarbus .........53

Sinibotia .........55

Sinibrama ........55

Sinigobio .........53

Sinilabeo ..........53

Siniperca .........994

Sinocyclocheilus .....53

Sinogastromyzon .....55

Sinogobius ........151

Sinohomaloptera .....55

Sinomyrus .........70

Sinosturio .........19

Siokunichthys ......83

Siphagonus ........163

Siphamia ..........99

Siphateles .........53

Siphonochromis ....120

Siphonognathus .....124

Siphonostoma ......83

Siphonostomus .....83

Siphostoma ........83

Sirembo ...........135

Sisor ...........60

Skagerakia ..........31

Skeponopodus .......141

Skiffia ............80

Sladenia ............170

Slatinia .........62

Smaragdus .........151

Smaris ...........112

Smarius .........112

Smecticus ...........94

Smiliogaster .........53

Smilogaster ........53

Smilogobius $\ldots \ldots \ldots 151$

Smithichthys ......130

Snithites .........23

Snellius ...........27

Snyderichthys .......53

Snyderidia ......134-135

Snyderina .........159

Soarus .............30

Soldatovia .........129

Solea KLEIN .......169

Solea Quensel .......169

Solea CASTB. .........166

Solea RAFNSQ. . . . . . . 169

Solea Cuv. ..........169

Solegnathus ........83

Soleichthys ........169

Solenichthus .......82

Solenichthys .......82

Solenognathus ......83

Solenomormyrus .....29
Solenophallus

.76

Solenostoma $\ldots . . . .82$

Solenostomus LACPD. . .82

Solenostomus

GRONOW

Solenostomus KLEIN ...73

Solenostomus BRWN. ..73 Solenostomus (G.)

GILL ............73

Soleonasus .........168

Soleotalpa .........168

Solivomer ...........32

Somersia ..........129

Somileptes ........55

Somniosus .........14

Somnisphinax .......13

Somnispinax ........13

Sonoda ...........27

Sorosichthys ........89

Sorsogona .........159

Sorubim .........63

Sorubimichthys .....63

Sosia ...........60

Sovichthys .......63

Spanius ...........169

Sparactodon ........102

Sparidentex .......111

Sparisoma .........123

Sparodon ...........I11

Sparopsis .......106-106

Sparosoma .........112

Sparosomus ........112

Sparus ..........112

Spathodus ........1i8

Spatularia ..........19

Spatuloricaria ......66

Spectrunculus .......135

Sperata ...........59

Sphaeramia ........99

Sphaerichthys .......144

Sphagebranchus ....7.71

Sphagemacrurus .....85

Sphagomorus .......165

Sphenanthias ........92

Sphenentogobius .....151

Sphenosargus .......112

Spheroides .........35

Sphoerina ........77

Sphoerodon .........110

Sphoeroides ..........35

Sphyra .............12

Sphyraena KLEIN ....777

Sphyraena RösE . . . .777

Sphyraena

BL. et SCHNDR. . . ...77

Sphyraenella .......777

Sphyraenocharax ....41

Sphyraenops .......99

Sphyrichthys .......12
Sphyrna ..........12

Sphyrnias ..........12

Spicara ..........112

Spilostomias ......28

Spilotichthys .......108

Spinachia .........76

Spinax (C.) C. et V. . 102

Spinax Cuv. ........13

Spinax BPTE. .......13

Spinibarbichthys .....53

Spinibarbus .........53

Spinipercina ........92

Spiniphryne ........172

Spiniraja ..........15

Spinirhombus

Spinivomer ........72

Spiniblennius .........129

Spinirhombus ......166

Spintherobolus .....41

Spirinchus .......25

Spirlingus ..........53

Spirlinus .........55

Spirobranchus .......144

Spixostoma ........41

Spondyliosoma .....114

Spratella .........23

Spratellicypris ......55

Spratelloides LACPD. ..23

Spratelloides BLKR. ...23

Spratellomorpha .....23

Sprattus ..........23

Springeria ..........16

Spurco ............157

Squalalburnus .......53

Squalidus ..........53

Squaliobarbus .......53

Squaliolus ..........14

Squalius ..........53

Squalogadus .......84

Squalomugil ........77

Squalraia ..........14

Squalraja ..........14

Squalus ...........13

Squamicreedia ......125

Squatina ............14

Squatinoraja ........15

Stagonotrigla .......157

Stalix ............124

Stanulus .........129

Stappersetta .......118

Stappersia .........118

Starksia ..........130

Starksina ........41

Stathmonotus .......131

Steatocranus .......118

Steatogenys .......4.43

Stegastes ..........120

Stegophilus .......64

Stegostoma .........10

Steindachnerella $\ldots . .84$ 
Steindachneria

G. et B.

Steindachneria

Eg. et Eg.

Steindachneridion

Steindachnerina

FWLR.

Steindachnerina

FDz.-Yz.

Steinegeria

Stelengicottus .......163

Stelengis ..........163

Stelgidonotus .......160

Stelgis Cramer ......163

Stelgistrops ........160

Stelgistrum .......160

Stellerina ..........163

Stellicarens ........109

Stellifer ...........109

Stelliferus .........109

Stellistius ..........158

Stemonidium .......72

Stemonosudis ........31

Stenatherina .......78

Stenesthes .........112

Stenobrachius .......32

Stenocaulus ........74

Stenodus .........25

Stenogobius .......151

Stenometopus ........35

Stenophus .........134

Stenoscolopsis ......108

Stenotomus .......112

Stephanoberyx .....89

Stephanolepis ....994-139

Stereolepidella .......92

Stereolepidella .......92

Stereolepis CsR. .....92

Stereolepis AYRS. ....92

Stereolepoides ......92

Sterledus ..........19

Sterleta ............19

Sterletus RAFNSQ. .....19

Sterletus

Bdt. et RTZBg. .....19

Sternarchella ........44

Sternarchogiton ......44

Sternarchorhampus ...44

Sternarchorhynchus ...44

Sternarchus .......44

Sternias .............160

Sternoptix ........27

Sternoptychides .....27

Sternoptyx …....27

Sternopygus .......44

Sternotremia .......73

Stethaprion ........41

Stethochaetus ......24

Stethojulis ........123

Stethopristes .......90
Stethopterus .......71

Stevardia .........41

Sthenopus .........159

Stichaeopsis .......131

Stichaeus ..........131

Sticharium ..........134

Stichonodon .......41

Stigmatogobius .....151

Stigmatonotus ......993

Stigmatophora .......83

Stigmatopora ........83

Stilbe ............53

Stilbiscus .........68

Stilbius ..........53

Stipecampus ........83

Stiphodon .........151

Stizostedion ..........101

Stizostethium ........101

Stelengicottus

Stlengis JD, et STKs. . . 160

Stoasodon ............17

Stokeliia ..........26

Stolephorus ......23-24

Stolothrissa .......23

Stomatorhinus ......29

Stomianodon ......27

Stomias ...........28

Stomiasunculus .....28

Stimioides ........28

Stomocatus ........45

Stomodon ..........85

Stomogobius .......151

Stoniella .........66

Strabo ...............76

Strabozebrias ........169

Strandichthys ......169

Strephon .........64

Strializa ..........77

Strinsia ..........86

Stromateoides .......142

Stromateus ........142

Strongylura ........74

Strophidon .......6.67

Strophiurichthys .....33

Strophodus .........106

Stupens ..........42

Stupidogobius .......151

Sturio Rafnsq. ....... 19

Sturio MüLr. . . . . . 19

Sturisoma .......66

Stygicola ...........135

Stygnobrotula ......135

Stygogenes .......64

Stylephorus ........87

Stylophorus . . . . . . 87

Stylophthalmella $\ldots \ldots 27$

Stylophthalmoides ...27

Stylophthalmus ...27-27

Stypodon ..........53

Suarenus ...........104
Sudis RAFNsQ. .......31

Sudis Cuv. .......29

Suezia .............121

Suezichthys ........122

Sufflamen .........138

Sufflogobius .......151

Suggrundus ........159

Suillus ..........122

Suprasinelepidichthys ..42

Suruga ............151

Sutorectus ..........10

Suttonia ..........96

Swainia ...........100

Syacium ............166

Syletophis .........71

Syletor ...........71

Symbolophorus ….32

Symboulichthys ....166

Symbranchus .......72

Symmetrurus .......53

Symphocles ........142

Symphodus ........122

Symphorus .........112

Symphurus .......170

Symphysanodon .....92

Symphysodon .......118

Symphysoglyphus ...101

Sympodoichthys ....86

Symproptopterus .....31

Sympterichthys .....171

Sympterygia ........15

Synagris KLEIN ....106

Synagris GTHR. ......106

Synagris $(K$.

BLKR. . . . . 106-112

Synagrops ..........99

Synanceia ...........157

Synanceichthys ....157

Synanceja ..........157

Synanchia .........157

Synancidium ........157

Synaphobranchus ....68

Synapteretmus .......32

Synaptolaemus .....42

Synaptura .........169

Synapturichthys .....169

Synbranchus .......72

Synchiropus .......127

Synchirus .........162

Synchismus ........12

Synclidopus .......169

Syncrossus ........55

Synechoglanis ......60

Synachogobius ......151

Synechopterus ......97

Syngnathoides .......83

Syngnathus ........83

Synistius ..........110

Synnema .........126

Synodontaspis .......10 
Svnodontis .......62

Synodus Gronow ....30

Synodus (G.) Scop. ...30

Sypterus ..........102

Syrictes ...........83

Syrraxis ...........17

Syrrhina ..........15

Syrrhothonus .......151

Systomus ..........53

\section{$-\mathrm{T}-$}

Taaningichthys ......32

Tachysurus .........57

Tactostoma .........28

Taenarichthys ......112

Taenarus ...........112

Taenia ............118

Taeniacara ..........118

Taenioconger ......6.69

Taenioides .........152

Taeniolabrus ........125

Taeniomembras .....78

Taenionema ......663

Taenionotus ........156

Taeniophis ........6.67

Taeniophorus .......33

Taeniopsetta ........166

Tueniotoca .........119

Taeniura ...........16

Tagusa ............129

Tahhmel ...........113

Taius .............112

Takenokius ........156

Takifugu ...........35

Talismania ........21

Tamanka ..........151

Tambra ............53

Tanakia ...........53

Tanakius ..........167

Tandanus .........58

Tandya ............124

Tanganicodus .......118

Tanganikallabes .....61

Tanichthys ........53

Tannayia ..........57

Tantalisor .........139

Taractes ..........90

Taractichthys .......90

Tarandichthys ......160

Tarletonbeania ......32

Tarphops ...........166

Tarpon .............19

Tarsites ............15

Tasmanogobius ......151

Tateichthys ........44

Tateurndia .......146

Tathicarpus ........171

Tatia .............57

Taumakoides .......96
Tauphysa ..........55

Tauredophidium ....21

Taurichthys .......115

Tauridea ..........162

Taurocottus ........162

Taurulus ..........162

Tautoga ...........123

Tautogolabrus ......22

Teixeirichthys ......120

Tekla .........129-130

Telara ............24

Teleogramma ....118-122

Teleotrema .......172

Telescopias ........99

Telescops ...........99

Telestes ..........53

Telipomis ..........97

Tellia ...........80

Telmatherina ......76

Telmatochromis ....118

Temeculina ........53

Temera ............17

Temnistia .........160

Temnocara ........164

Temncidon ...........102

Tengujei $\quad . \ldots \ldots \ldots 15$

Tentoriceps ........142

Tenualosa ........25

Tephraeops .........114

Tephrinectes ........166

Tephritis ...........166

Terapon ...........108

Teratichthys .......73

Teratorhombus .....166

Teretulus .........45

Tetrabrachium .....171

Tetrabranchus .....72

Tetracentrum ......97

Tetrachaetodon .......115

Terades ...........121

Tetradrachmum ....120

Tetragonopterus

Klein ...........115

Tetragonopterus Cuv. .. 41

Tetragonopterus (K.)

BLKR. ...........115

Tetragonurus .......143

Tetranarce ..........17

Tetranematichthys ....57

Tetranesodon .......57

Tetraodon ...........35

Tetrapleurodon .......7

Tetrapturus .........141

Tetraroge .........156

Tetrodon ............35

Tetronarce ............17

Tetronarcine ........17

Tetroras ............10

Tetrosomus ..........33

Teuthis a. L. .......137
Teuthis LINNÉ .......137

Teuthis BRwn. .....137

Teuthys ..........137

Teutis ...........137

Tewara ...........125

Thaerodontis ......67

Thaigobiella ........151

Thalassinus .........12

Thalassogobius .....151

Thalassoklephtes ....13

Thalassoma ........123

Thalassophryne .....153

Thalassorhinus .....12

Thalassosteus .......74

Thallassothia .......153

Thaleichthys .......25

Thalliurus ........123

Thamnaconus ......139

Tharbacus .........153

Thaumastomias ....28

Thaumatichthys ....172

Thayeria E. 1907 ....41

Thayeria E. $1908 \ldots 44$

Thecapteryx ,......35

Thecopsenes .......143

Thecopterus .......162

Themistocles ........146

Theragra .........86

Therapaina ........115

Therapon ..........108

Theraps .........118

Therobromus ......26

Thinnus ..........140

Thoburnia ........45

Tholichthys .......115

Thoracatherina ......78

Thoracocharax .....44

Thorichthys .......118

Thorophos ........27

Threpterius ........118

Thrissa RAFNSQ. .....23

Thrissa Cuv. ......24

Thrissina ........24

Thrissobrycon ......44

Thrissocharax ......43

Thrissochromis .....121

Thrissocles ........24

Thrissomimus ......25

Thryssa .........24

Thunnus .........140

Thymalloides .......25

Thymallus LINCK ....25

Thymallus Cuv. .....25

Thynnichthyina ......53

Thynnichthys BLKR. ..53

Thynnichthys GGLI. ..140

Thynnus GRONOW ...104

Thynnus Cuv. .......140

Thynnus BRwn. ......140

Thyreoconger ......69 


\begin{tabular}{|c|c|c|}
\hline & 127 & Xenodermichthys \\
\hline Vanmanenia ..... & & Xenodexia ..... \\
\hline Vanstraelenia $\quad \ldots \ldots 169$ & W & Xenodon \\
\hline Varicogobio ....... 54 & & Xenognathus \\
\hline Varocorhinus ..... & Waitea & Xenogobius \\
\hline Variola ........... & Waiteina . & Xenogramma ..... \\
\hline Vastres & Waiteopsis & julis \\
\hline Vauclusella ........130 & Wakiyus & Xenolepidichthys. \\
\hline Vegetichthys $\ldots \ldots \ldots 106$ & Walbaumina $\ldots . .$. & Xenomelaniris ..... \\
\hline elambassis ........97 & Wallago ........... & uugil $\quad \ldots . .$. \\
\hline elasia $\ldots \ldots \ldots \ldots$. 8 & Wallagonia $\ldots \ldots \ldots 58$ & cystax \\
\hline onymus $\quad \ldots \ldots 127$ & $\ldots \ldots \ldots 140$ & aystus GTHR. \\
\hline elifer ..........8. 87 & …... & aystus LTKN. \\
\hline elifracta $\ldots \ldots \ldots 166$ & Warmizichthys .....96 & horus $\ldots . .$. \\
\hline itor $\ldots \ldots \ldots \ldots 162$ & Warreenula ........27 & $\ldots \ldots \ldots 81$ \\
\hline$c a \ldots \ldots \ldots$ & Watasea ... & 129 \\
\hline ssa $\quad \ldots \ldots \ldots .85$ & Weberogobius ......151 & $X$ \\
\hline 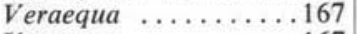 & Wertheimeria ......557 & ithys \\
\hline$\ldots \ldots \ldots$ & Wetmorella ...... & chthys \\
\hline er $\ldots \ldots \ldots$ & Whitleyia & $s \ldots .$. \\
\hline ectes $\ldots . . \ldots$ & Whitleyidea ....... & \\
\hline $\operatorname{rax} \ldots \ldots$ & Whitleyina ....... & $X$ \\
\hline ndum $\ldots . .$. & Wilkesina & hys \\
\hline lus $\ldots . . . . . .$. & Winteria & \\
\hline$a \quad \ldots \ldots \ldots \ldots \ldots 70$ & Woodsia & chichthys \\
\hline$\ldots \ldots \ldots$ & $\ldots \ldots \ldots$ & ops $\ldots .$. \\
\hline ilus $\ldots \ldots$.... & & ... \\
\hline to $\ldots \ldots \ldots$ & 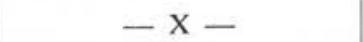 & $\ldots$. \\
\hline alus $\quad \ldots$. & & apia \\
\hline us $\ldots \ldots \ldots$. & ion & $\ldots \ldots$ \\
\hline la $\ldots \ldots \ldots$. & chthys & .80 \\
\hline is $\ldots \ldots \ldots$ & cles ....... & rycon ... \\
\hline$\ldots \ldots \ldots$ & 69 & harax ... \\
\hline$\ldots \ldots \ldots$ & niates .......4 41 & $s \quad \ldots \ldots \ldots$ \\
\hline $\operatorname{anx} \ldots$ & assis $\ldots . . .997$ & 137 \\
\hline$\cdots \cdots$ & .93 & is $\ldots \ldots \ldots$. \\
\hline gla $\ldots .$. & erina $\ldots \ldots \ldots \ldots 78$ & 132 \\
\hline ....... & $m \ldots \ldots \ldots 54-80$ & \\
\hline & ulis & thys \\
\hline 0 & odon $\ldots \ldots \ldots .74$ & 131 \\
\hline 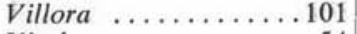 & tmus $\ldots . . .$. & 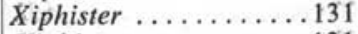 \\
\hline & iys $\ldots . . \ldots$. & \\
\hline & $a \quad \ldots \ldots \ldots \ldots 80$ & $u s \quad \ldots \ldots \ldots$. \\
\hline ria .... & imus $\ldots . .$. & $\operatorname{arax} \ldots .$. \\
\hline im & & ilus \\
\hline & rbus ....... & dus $\ldots . .$. \\
\hline & glossus $\ldots$. & \\
\hline Ila & ites $\ldots .$. & iphus \\
\hline$a \ldots \ldots$. & $\ldots \ldots \ldots 66$ & ynchus ... \\
\hline & phalus $\quad \ldots .85-132$ & $m a$ SPIX ... \\
\hline & & na Agsz. \\
\hline arus & harax & otheca $\quad . \ldots \ldots 142$ \\
\hline 5 & zeilichthys . & ops . \\
\hline & .163 & ys \\
\hline 04 & hromis ........118 & Xurel \\
\hline lus & Xenoclarias & \\
\hline Sis & 66 & hen \\
\hline$a$ VALMT. & $X$ & \\
\hline Ia GARM. ...... 9 & cys $\ldots \ldots \ldots$. & \\
\hline us $\ldots \ldots \ldots$. & & \\
\hline
\end{tabular}




\begin{tabular}{|c|c|}
\hline & \\
\hline$m a$ & emus \\
\hline$\ldots .$. & thias \\
\hline rus $\quad \ldots \ldots \ldots$ & Zalarges \\
\hline$\ldots \ldots \ldots \ldots$ & Zalembius .... \\
\hline$u s \ldots \ldots$ & Zalescopus ... \\
\hline nia $\ldots . . \ldots$ & utes .... \\
\hline urys .........167 & Zali. \\
\hline$\ldots \ldots$ & 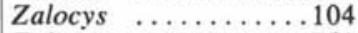 \\
\hline erca .... & nus $\ldots .$. \\
\hline iorus... & \\
\hline lites .... & \\
\hline is $\ldots \ldots$. & \\
\hline & $S$ \\
\hline & to \\
\hline hys & \\
\hline & (C.) C. \\
\hline us $\ldots$ & mis $\ldots$. \\
\hline$\ldots \ldots$ & ois ... \\
\hline & $a \ldots$ \\
\hline .8 & tias ... \\
\hline & \\
\hline & $Z$ \\
\hline & iias \\
\hline thys & $x \quad \ldots$ \\
\hline & inius. \\
\hline 4 & $\ldots$ \\
\hline 121 & oma ... \\
\hline lus & otris. \\
\hline ..... & $\ldots \ldots$ \\
\hline & \\
\hline $\mathrm{Z}-$ & $\cdots$ \\
\hline & \\
\hline$\cdots$ & pterus \\
\hline & \\
\hline
\end{tabular}

Zenodon ............138

Zenopsis .........90

Zeodrius ..........119

Zesticelus ............162

Zestichthys ........133

Zestidium .........109

Zestis .............109

Zeugopterus .......166

Zeus ..............90

Zev $\quad \ldots \ldots \ldots \ldots \ldots 10$

Zevaia ............169

Zezera ............54

Zingel .............101

Ziphasia ...........132

Ziphotheca ........142

Zoarces ............133

Zoarchias .........131

Zoarcites ..........133

Zonichthys ........104

Zonogobius ........151

Zonophichthus ......71

Zonoscion ...........109

Zoogoneticus .......80

Zopa .............55

Zophendum .........54

Zoramia ............99

Zostericola ..........151

Zosterisessor ........151

Zunasia ..........23

Zungaro ..........63

Zungaropsis .......63

Zygaena ..........12

Zygobatis ..........17

Zygogaster ........41

Zygonectes .......80

Zyphothyca $\ldots \ldots \ldots 142$ 